\title{
Tragverhalten von \\ Mauerwerkswänden unter Druck und Biegung
}

\section{Working Paper}

Author(s):

Furler, René

Publication date:

1981

Permanent link:

https://doi.org/10.3929/ethz-a-000226922

Rights / license:

In Copyright - Non-Commercial Use Permitted

Originally published in:

Bericht / Institut für Baustatik und Konstruktion ETH Zürich 109 
Tragverhalten von Mauerwerkswänden unter Druck und Biegung

René Furler 


\section{CIP-Kurztitelaufnahme der Deutschen Bibliothek}

\section{Furler, René:}

Tragverhalten von Mauerwerkswänden unter

Druck und Biegung / von René Furler.-

Basel; Boston; Stuttgart: Birkhäuser, 1981.

Bericht / Institut für Baustatik und Konstruktion

ETH-Zürich, Nr. 109)

ISBN 3-7643-1257-2

NE: Institut für Baustatik und Konstruktion

$<$ Zürich $>$ : Bericht/Institut für...

Nachdruck verboten.

Alle Rechte, insbesondere das der Übersetzung in fremde Sprachen und der Reproduktion auf photostatischem Wege oder durch Mikrofilm, verboten. 


\section{Tragverhalten von Mauerwerkswänden unter Druck und Biegung}

von

Dr. sc. techn. René Furler

Institut für Baustatik und Konstruktion

Eidgenössische Technische Hochschule Zürich 


\section{Vorwort}

Der Backstein ist wohl das älteste künstlich hergestellte Baumaterial. Seine Anwendung beruhte bis vor wenigen Jahrzehnten allein auf Erfahrung. Alle heute gültigen Bemessungsverfahren für Backsteinbauten basieren im wesentlichen auf der Elastizitätstheorie mit einer Begrenzung durch zulässige Spannungen. Die Unzulänglichkeiten solcher Verfahren sind zwar allgemein bekannt. Trotzdem wird erst in jüngster Zeit versucht, das unelastische Verhalten bis zum Bruch in die Bemessung einzubeziehen.

Die vorliegende Arbeit hat Herr R. Furler im Rahmen eines Forschungsprojektes über das Tragverhalten von Mauerwerkswänden unter Druck und Biegung als Dissertation verfasst. Er hat ein theoretisches Verfahren zur Berechnung der Verformungen und der Traglast solcher Wände hergeleitet und mit Versuchsergebnissen kritisch verglichen. Schliesslich schlägt er ein praktisches Bemessungsverfahren vor.

Die Ergebnisse dieser Arbeit finden im kürzlich veröffentlichten Entwurf der SIA-Richtlinie 177/1 "Bemessung von Backstein- und Kalksandstein-Mauerwerkswänden auf die Grenzzustände der Tragfähigkeit und der Gebrauchsfähigkeit" ihre Anwendung. 


\section{Inhal tsverzeichnis}

Seite

1. PROBLemstellung UND ZIELSETZUNG

2. LITERATURUEBERSICHT 3

3. TECHNISCH-PHYSIKALISCHE EIGENSCHAFTEN VON MAUERWERK

3.1 Ueberblick. 5

3.2 Verhalten von Mauerwerk unter axialer Belastung 6

3.3 Stoffgesetz von Backsteinmauerwerk 8

4. MOMENTEN / EXZENTRIZITAET - KRUEMMUNGS-VERHALTEN,
INTERAKTIONSBEZIEHUNG

4.1 Ueberblick 14

4.2 Linear elastisches Material ohne Zugfestigkeit. 15

4.3 Linear elastisches Material mit beschränkter Zugfestigkeit 22

4.4 Linear elastisch-ideal plastisches Material ohne Zugfestigkeit 26

4.5 Einfluss der Lochung 29

4.6 Folgerungen 33

5. VERGLEICH VON EXPERIMENTELLEN UND RECHNERISCHEN $\&-\Phi-K U R V E N$ VON BACKSTEINMAUERWERK

5.1 Experimentelle e-\$-Kurven 34

5.2 Stoffgesetz für Backsteinmauerwerk 37

5.3 Gegenüberstellung von experimentellen und rechnerischen

e-Ф-Kurven 38

6. TRAGVERHALTEN VON AXIAL BELASTETEN MAUERWERKSWAENDEN 44

6.1 Ueberblick 44

6.2 Analytische Lösungen $\quad 47$

6.3 Numerische Verfahren 65

6.4 Starrkörper-Federmodell 83

6.5 Einfluss der Zugfestigkeit und der Lochung auf das Trag- und Bruchverhalten 88

7. WAND - DECKEN - SYSTEM 92

7.1 Ueberblick 92

7.2 Zusammenwirken von Wänden und Decken 93

$\begin{array}{ll}7.3 \text { Subsysteme } & 100\end{array}$

8. BEMESSUNG VON MAUERWERKSWAENDEN 104

8.1 Ueberblick 104

8.2 Bemessungskonzept $\quad 105$

8.3 Nachweis der Bruchsicherheit 106

8.4 Nachweis der Gebrauchsfähigkeit. 108

8.5 Berücksichtigung von Kriech-, Schwind- und Temperaturver-

formungen 109

$\begin{array}{ll}\text { 8.6 Auflagerdrehwinkel } \vartheta_{D} \text { der Decke. } & 110\end{array}$ 
ANHANG A1: Mauerwerksgattungen

ANHANG A2: Linear elastisches Material mit Zugfestigkeit

ANHANG A3: Linear elastisch-ideal plastisches Material ohne Zugfestigkeit

ANHANG A4: Krümmungs- und Interaktions-Beziehungen bei zwei extremen Lochungen $A$ und $B$

ANHANG A5: Bemessungsverfahren für Backstein- und KalksandsteinMauerwerk unter Axiallast und Biegung 


\section{Problemstellung und Zielsetzung}

In Bild 1.1 ist ein im Hochbau übliches Tragsystem dargestellt. Die Stahlbetondecken sind im allgemeinen ganz oder teilweise in die Mauerwerkswände eingebunden (Bild 1.1 b). Bei erhöhten differentiellen Schwind- und Temperaturverformungen werden zwischen Wand und Dachdecke vielfach Gleitlager eingebaut (Bild 1.1 a).

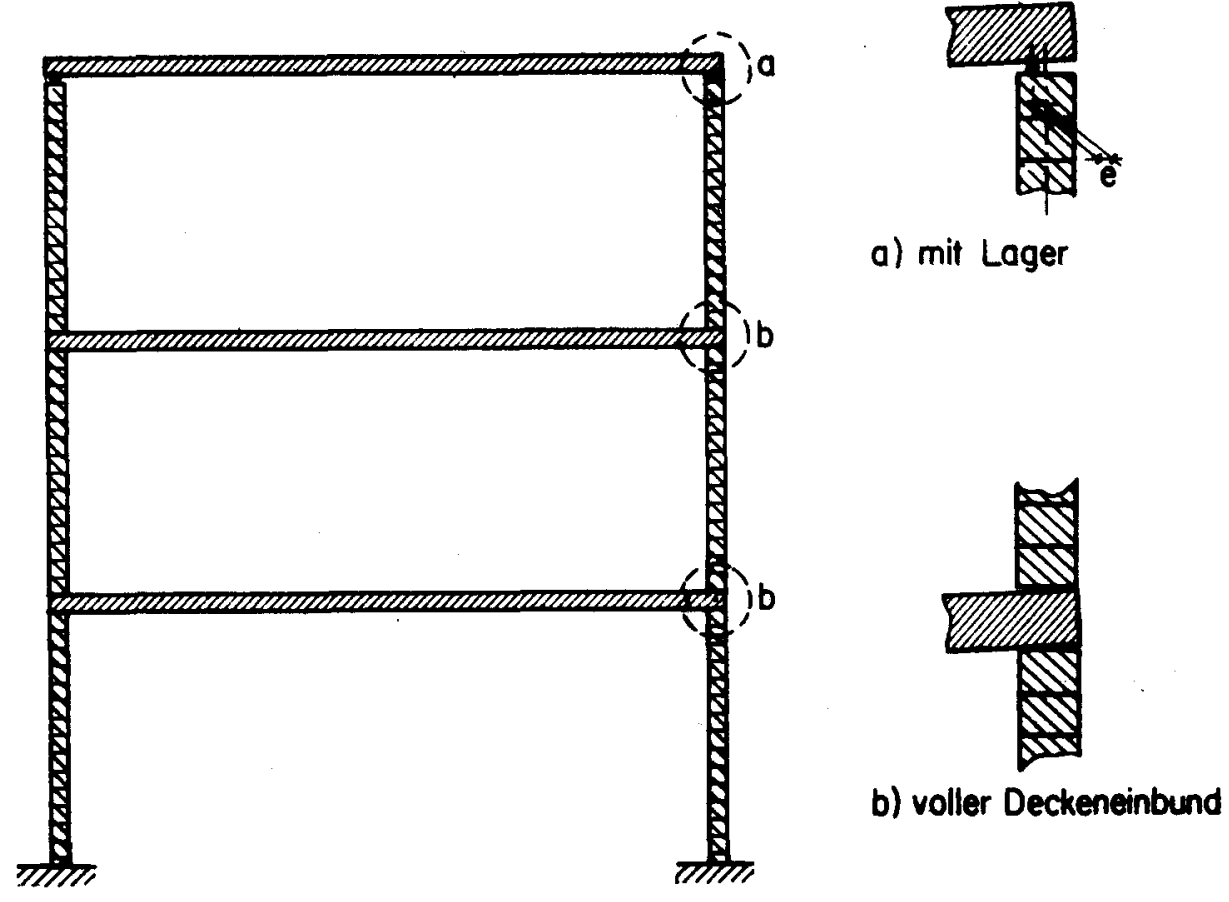

\section{Bild 1.1: Wandbeanspruchungen}

Diese beiden Deckenlagerungen sind für die Beanspruchung der Mauerwerkswände von Bedeutung. Im Falle des Deckeneinbundes ergibt sich durch das Zusammenspiel von Wand und Decke eine Einspannwirkung, und die Axiallast stellt sich bezüglich der Wandaxe exzentrisch. Die Grösse der Exzentrizität ist von der Einspannung bzw. von der Verdrehung des Deckenauflagers abhängig. Demgegenüber wird durch ein Gleitlager die Exzentrizität der Axiallast fixiert.

Währenddem das Tragverhalten derartig beanspruchter Mauerwerkswände (Exzentrizität konstant) in zahlreichen experimentellen und theoretischen Arbeiten abgeklärt wurde, ist das Tragverhalten von Mauerwerkswänden, die durch darin eingebundene Decken beansprucht werden, bis heute noch ungenügend abgeklärt. Bei solchen Wänden sind die Exzentrizitäten durch die Konstruktion nicht fixiert, sie sind von der Grösse der Einspannung bzw. der Auflagerverdrehung der Decke abhängig.

Zwischen den Wandend- bzw. Deckenauflagerverdrehungen $\left(\vartheta, v_{D}\right)$ und den Exzentrizitäten e der Axiallast bestehen Beziehungen, die durch die Grösse der 
Axiallast $P$, die Wandschlankheit sowie die Materialeigenschaften und Querschnittsform der Wand (Lochung) mitbestimmt werden (Bild 1.2).

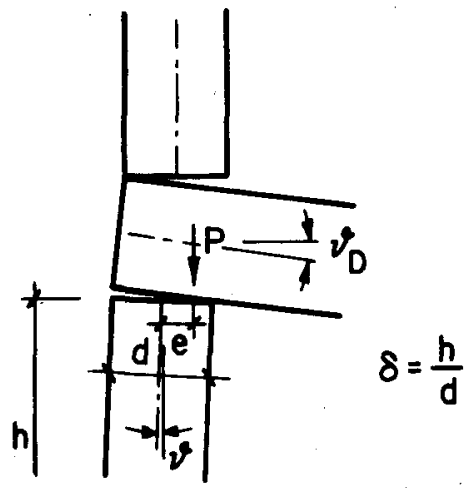

Bild 1.2: $P-e-v-v_{D}-\delta$

Das Ziel der vorliegenden Arbeit besteht darin, diese Zusammenhänge unter Einbezug der angegebenen Einflussgrössen zu untersuchen und damit das Tragverhalten von axial belasteten Mauerwerkswänden bis zum Bruch zu beschreiben. Im weiteren soll das Zusammenspiel von Wänden und Decken im Rahmensystem dargestellt und eine Methode zur einfachen rechnerischen Behandlung angegeben werden.

Die vorliegenden theoretischen Untersuchungen wurden im Rahmen des Forschungsprojektes "Rotationsfähigkeit von Mauerwerk" durchführt. Daneben wurde das Trag- und Bruchverhalten von Backstein- und Kalksandsteinwänden experimentell abgeklärt und in zwei Versuchsberichten [7], [8] beschrieben. Die experimentellen und theoretischen Arbeiten bildeten die Grundlage für die Ausarbeitung eines neuartigen Bemessungsverfahrens für Mauerwerkswände unter Druck und Biegung. Es ist im Anhang A5 dargestellt und im 8. Kapitel erläutert. Entsprechend der heutigen Entwicklung in anderen Bauweisen (Stahl- und Stahlbetonbau) müssen bei der Bemessung gesonderte Nachweise für die Bruchsicherheit einerseits und die Gebrauchsfähigkeit andererseits durchgeführt werden. 


\section{Li teraturübersicht}

Die ersten systematischen Untersuchungen über die Festigkeit von axial belasteten Mauerwerkswänden wurden in der Schweiz in den vierziger Jahren von Haller begonnen. In [14] veröffentlichte er erste experimentelle Resultate und verglich diese mit rechnerisch ermittelten Werten.

Die Differentialgleichung der ausgebogenen Wandaxe wurde von Angervo 1954 [2] für ein linear elastisches Material ohne Zugfestigkeit und einen Rechteckvollquerschnitt gelöst. Die Beziehung zwischen Wandendverdrehung, Wandendexzentrizität, Axiallast und Wandschlankheit war damit hergestellt.

Viele Arbeiten befassen sich mit den Einspannverhältnissen in Wand-DeckenSystemen sowie mit dem Tragverhalten von Rahmensystemen.

Im Hinblick auf ein grosses Bauvorhaben in Sydney wurde das Zusammenwirken von Backsteinwänden und darin eingespannten Decken untersucht [1]. An einem Modellversuch in natürlicher Grösse wurde festgestellt, dass die experimentell bestimmten Exzentizitäten wesentlich geringer waren als die normenmässig bestimmten Werte. Dementsprechend überstieg die Wandtragfähigkeit die Normentragfähigkeit deutlich.

Gegenstand einer Arbeit von Sahlin [29] war die experimentelle Untersuchung der Beziehung zwischen Biegemoment und Fugendrehwinkel der Verbindung Wand und Decke. Es zeigte sich, dass die Steifigkeit dieser Verbindung stark von den Einbundverhältnissen abhängt.

Maurenbrecher und Hendry [24] untersuchten ebenfalls die Verbindung Wand und Decke. In eine axial belastbare Backsteinwand war in halber Höhe eine Kragplatte in Stahlbeton eingebunden, über die mittels einer Pressenkraft ein Biegemoment eingetragen werden konnte. Die Begrenzung des maximalen Biegemomentes ergab sich dabei je nach Grösse der Axiallast durch:

a) Biege - und Schubversagen der Kragplatte

b) Bruch der Backsteinwand im Bereich des Platteneinbundes

c) Abheben des oberen Wandabschnittes infolge zu geringer Axiallast.

Im Bereich kleiner Biegemomente war die Beziehung zwischen dem eingetragenen Biegemoment und der Auflagerverdrehung der Kragplatte linear und praktisch unabhängig von der Axiallast. Bei grösseren Verdrehungen zeigt sich dann jedoch der Einfluss der Axiallast deutlich $(a, b, c)$.

Colville und Hendry untersuchten den Einfluss der Axiallast auf das Wandeinspannmoment [5]. An einem symmetrischen Rahmensystem konnte die axiale Last 
in den beiden seitlich angeordneten Backsteinwänden unabhängig von der mittels Pressen in den Drittelspunkten der beiden Stahlbetondecken aufgebrachten Deckenlast variiert werden. Mit zunehmender Axiallast in der Backsteinwand nimmt das Wand-bzw. Deckeneinspannmoment zunächst fast linear zu. Im Bereich grösserer Axiallast schwächt sich dieser Einfluss stark ab und die Einspannverhältnisse sind praktisch unabhängig von der Axiallast.

In Arbeiten von Sinha/Hendry [36] und Germanino/Macchi [10] wurden an einem zwei- bzw. dreistöckigen Rahmensystem die Verformungen und Schnittkräfte experimentell bestimmt und mit den Resultaten einer elastischen Rahmenrechnung verglichen. In beiden Fällen ergaben sich im obersten Stockwerk deutliche Abweichungen. In den unteren Stockwerken stimmten die experimentellen und rechnerischen Resultate hingegen gut überein. 


\section{Technisch-physikalische Elgenschaften von Mauerwerk}

3. 1 Ueberblick

Im Mauerverband werden die einzelnen Steine nach bestimmten Regeln zu einem monolithischen Körper verbunden (Bild 3.1). Im Einsteinmauerwerk (Läuferoder Binderverband) wird die Mauerdicke durch einen Stein gebildet. Das einschalige Mauerwerk wird als Einstein-oder Verbandmauerwerk ausgebildet und das zweischalige Mauerwerk besteht aus zwei Einsteinmauerwerkswänden, die durch einen Luftspalt oder eine Isolationsschicht getrennt sind.

Einsteinmauerwerk:

im Löuferverband

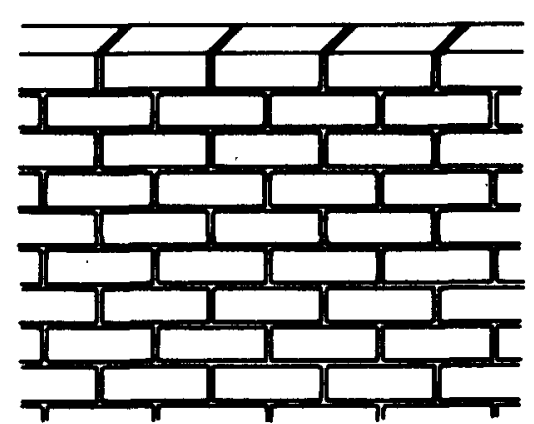

im Binderverband

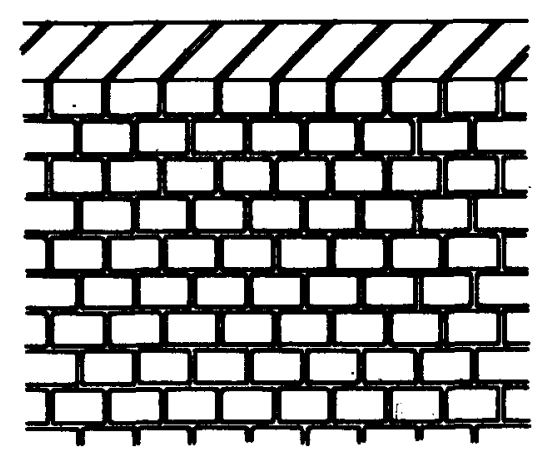

Verbandmauerwerk:

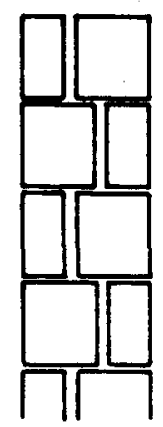

Zweischalenmquerwerk:

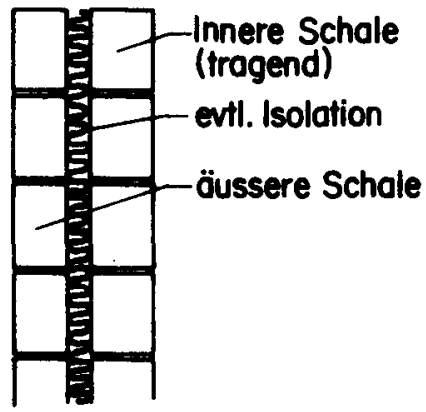

\section{Bild 3.1: Verbandarten}

Im konstruktiven Mauerwerksbau werden die Steine so angeordnet, dass alle. Stossfugen durch Steine in der jeweils darüber liegenden Schicht überdeckt werden, so dass allseitig eine Verzahnung der Steine entsteht. Die zwischen den Steinen liegenden Mörtelfugen (Stoss- und Lagerfugen) haben die Aufgabe die Steine einzubinden, die Kräfte von Stein zu Stein gleichmässig zu übertragen. Unebenheiten und Massdifferenzen der Steine auszugleichen und allfälige Spannungskonzentrationen zu vermindern. Das Tragverhalten von Mauer- 
werk unter axialer Belastung ergibt sich aus den Eigenschaften und dem Zusammenwirken der beiden Komponenten Stein und Mörtel. Es wird im Folgenden phänomenologisch und modellhaft beschrieben. Im weiteren sollen einige Spannungs-Stauchungs-Kurven von Backsteinmauerwerk angegeben und deren hauptsächlichsten Einflussgrössen beschrieben werden. Stoffgesetze anderer Mauerwerksgattungen wie Kalksandstein-, Zementstein- und Gasbetonstein-Mauerwerk sind in der Literatur praktisch nicht vorhanden.

\subsection{Verhalten von Mauerwerk unter axialer Belastung}

\subsubsection{Phänomenologische Betrachtung}

Wird eine Mauerwerkswand zentrisch bis zum Bruch belastet, so beobachtet man in der Regel, dass bei ca. 65 bis $85 \%$ der Bruchlast vertikal verlaufende Risse auftreten. Bei weiterer Laststeigerung weiten sich diese Risse aus. Der Bruch der Wand erfolgt schliesslich durch lokales Ausknicken dieser Einzelsäulen oder durch Materialzerstörung. Letzteres äussert sich durch Abbröckeln von Steinteilchen resp. Mörtel und durch Abplatzungen grösserer Steinschalen. In Bild 3.2 ist ein typisches Bruchbild einer derartig belasteten Wand dargestelit [30].

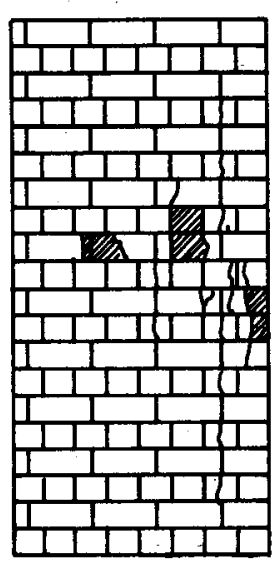

Vorderseite

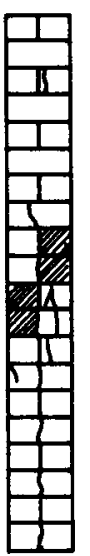

Bruchibild
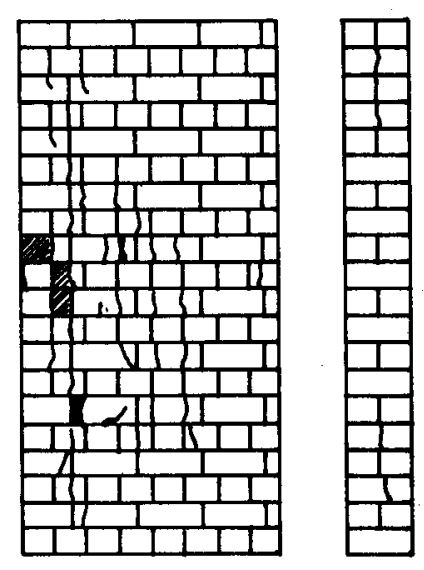

Rückseite

\section{Bild 3.2: Typisches Bruchbild}

\subsubsection{Empirische Ansätze für die Mauerwerksfestigkeit}

Mauerwerksversuche der obigen Art werden seit mehr als fünfzig Jahren durchgeführt. Sie dienen hauptsächlich der Bestimmung der Mauerwerksfestigkeit $\sigma_{u}$. Auf der Basis von Versuchsresultaten wurden viele empirische Ansätze zur Bestimmung von $\sigma_{u}$ gemacht. Kirtschig [20] überprüfte deren 28 . Sie basieren nicht auf einer modelihaften Vorstellung des Kräftespiels im Mauerwerk. Sie vermögen aber innerhalb bestimmter Parametergrenzwerte die Mauerwerksfestigkeit zufriedenstellend vorauszusagen. Obwohl es sich gezeigt hat, dass $\sigma_{u}$ von vielen Einflussgrössen abhängig ist, werden meistens nur Stein- $\left(\beta_{S}\right)$ und 
Mörteldruckfestigkeiten $\left(\beta_{M}\right)$ als Parameter verwendet. Nachstehende Ansätze von Haller und Hansson sollen stellvertretend für alle weiteren empirischen Formeln verstanden werden [30]:

Haller: $\quad \sigma_{U}=\left(\sqrt{1+0.15 \cdot \beta_{S}}-1\right) \cdot\left(8+0.057 \cdot \beta_{M}\right)$

Hansson: $\quad \sigma_{U}=2 \cdot \sqrt{\beta_{S}}+3 \cdot \sqrt{\beta_{M}}$

In Bild 3.3 sind die Mauerwerksfestigkeiten $\sigma_{u}$ in Abhängigkeit der Steinund Mörteldruckfestigkeit (für $\beta_{M}$-Werte von $5.0,10.0$ und $20.0 \mathrm{~N} / \mathrm{mm}^{2}$ ) aufgetragen.

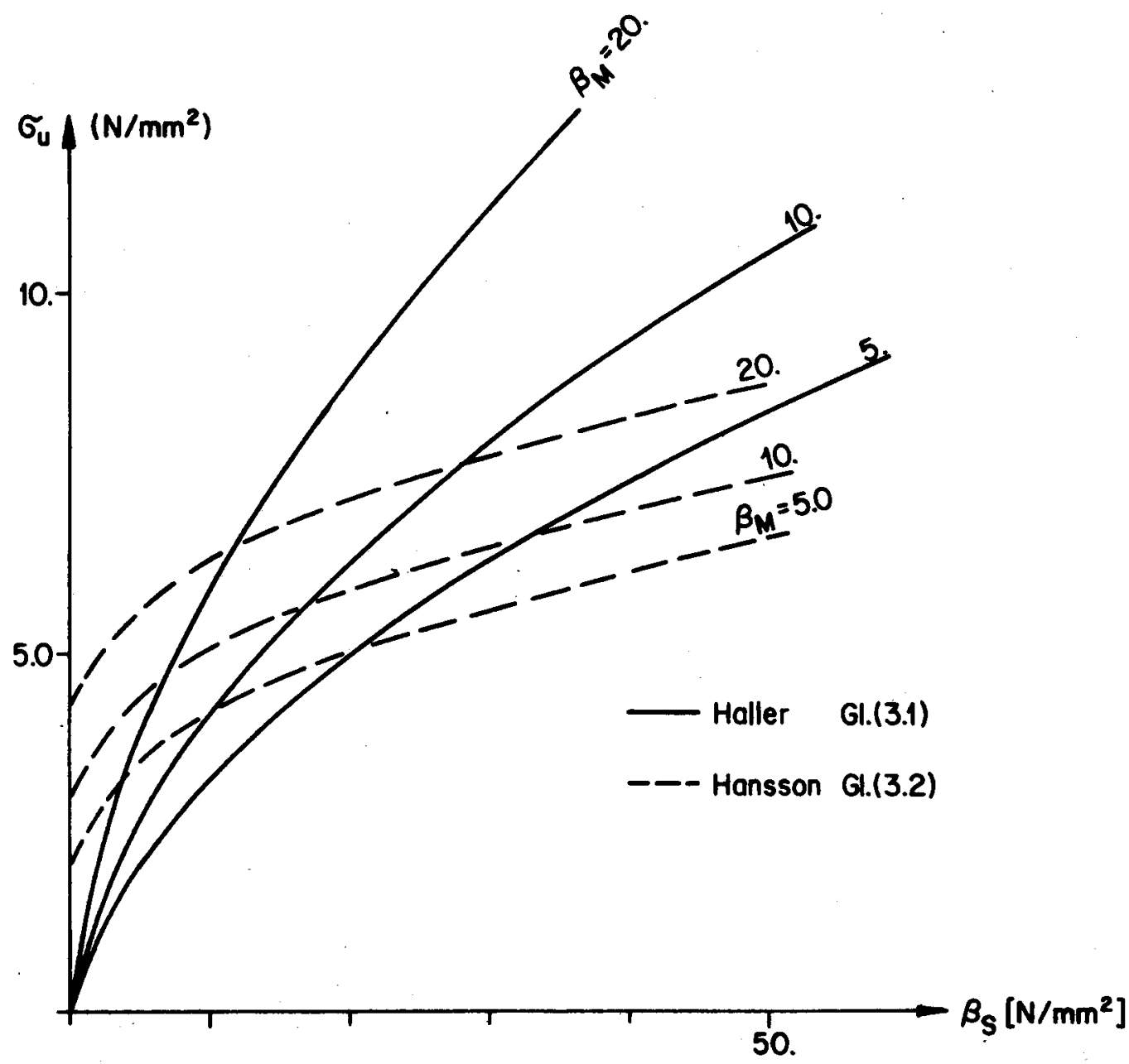

Bild 3.3: Empirische Ansätze für $\sigma_{u}$

\subsubsection{Model lhafte Betrachtung}

Bei axialer Belastung werden Stein und Mörtel axial gestaucht und gleichzeitig quer zur Kraftwirkung gedehnt [16]. Sofern die Querdehnungen der beiden Komponenten Stein und Mörtel gleich gross sind, treten keine Veränderungen der einaxialen Spanṇungszustände auf. Die Querdehnung von Mörtel ist in der Regel jedoch grösser als diejenige des Steins und zudem normalspannungs- 
abhängig. Wird nun angenommen, dass an der Kontaktfläche von Stein und Mörtel keine relativen Verschiebungen auftreten, so wird einerseits der Mörtel in der Querdehnung behindert und andererseits der Stein durch die entsprechenden Reaktionen quer zur Kraftwirkung zusätzlich gedehnt. In Wandrichtung wird die Querdehnung von Stein und Mörtel behindert.

Bei sehr langen Wänden kann deshalb von einem ebenen Verformungszustand gesprochen werden. Liegt der betrachtete Mauerausschnitt im Bereich des Wandendes, so können auch in Wandrichtung Querdehnungen auftreten.

Dieses Zusammenwirken von Stein und Mörtel im Mauerverband unter axialer Belastung führt zu zwei mehraxialen Spannungszuständen. Im Stein treten neben die Druckspannungen aus äusserer Belastung Querzugsspannungen und im Falle des ebenen Verformungszustandes einerseits Querzugspannungen und andererseits Querdruckspannungen. Der Mörtel wird allseitig durch Druckspannungen beansprucht.

\subsection{Stoffgesetz von Backsteinmauerwerk}

Das Stoffgesetz bzw. das Spannungs-Stauchungs-Verhalten von Mauerwerk wird üblicherweise aus Versuchen an zentrisch belasteten Kleinkörpern ermittelt. Kleinkörper sind gedrungene Wandkörper, deren Höhe aus ca. drei bis sechs Steinschichten besteht.

Die breit gefächerten Eigenschaften der Mauersteine und des Mauermörtels sowie die vielfältigen Möglichkeiten bei der Wahl der beiden Komponenten führen zu grossen Unterschieden im Spannungs-Stauchungs-Verhalten von Mauerwerk. Zudem werden die Spannungen in der Regel auf den Bruttowandquerschnitt bezogen, womit der Lochflächenanteil Lo bei der Spannungsberechnung nicht berücksichtigt wird.

Vollständige Spannungs-Stauchungs-Kurven von Mauerwerk sind nur in geringer Anzahl veröffentlicht worden. Die meisten Verformungsmessungen wurden vor dem Erreichen der Bruchlast abgebrochen und dienten oft nur zur Bestimmung des E-Moduls. Zentrisch bis zum Bruch belastete Wände bzw. Kleinkörper verlieren ihr. Tragvermögen häufig recht überraschend und unter Ausbrüchen von Maversteinen oder Teilen davon, so dass die Messeinrichtungen oft aus Sicherheitsgründen bei Lasten, die deutlich unter der Bruchlast lagen, entfernt wurden. Zudem war das Verformungsverhalten bis zum Bruch früher kaum von Interesse.

Eine Untersuchung von Kirtschig [20] führte zum Ergebnis, dass die Anzahl der zur Verfügung stehenden Versuchsergebnisse zu gering war, um einheitliche Spannungs-Stauchungs-Kurven für verschiedene Mauerwerksgattungen und -qualitäten anzugeben. In Bild 3.4 ist eine Auswahl von Spannungs-Stauchungs-Kurven $(\sigma-\varepsilon)$ für Backsteinmauerwerk dargestellt. 


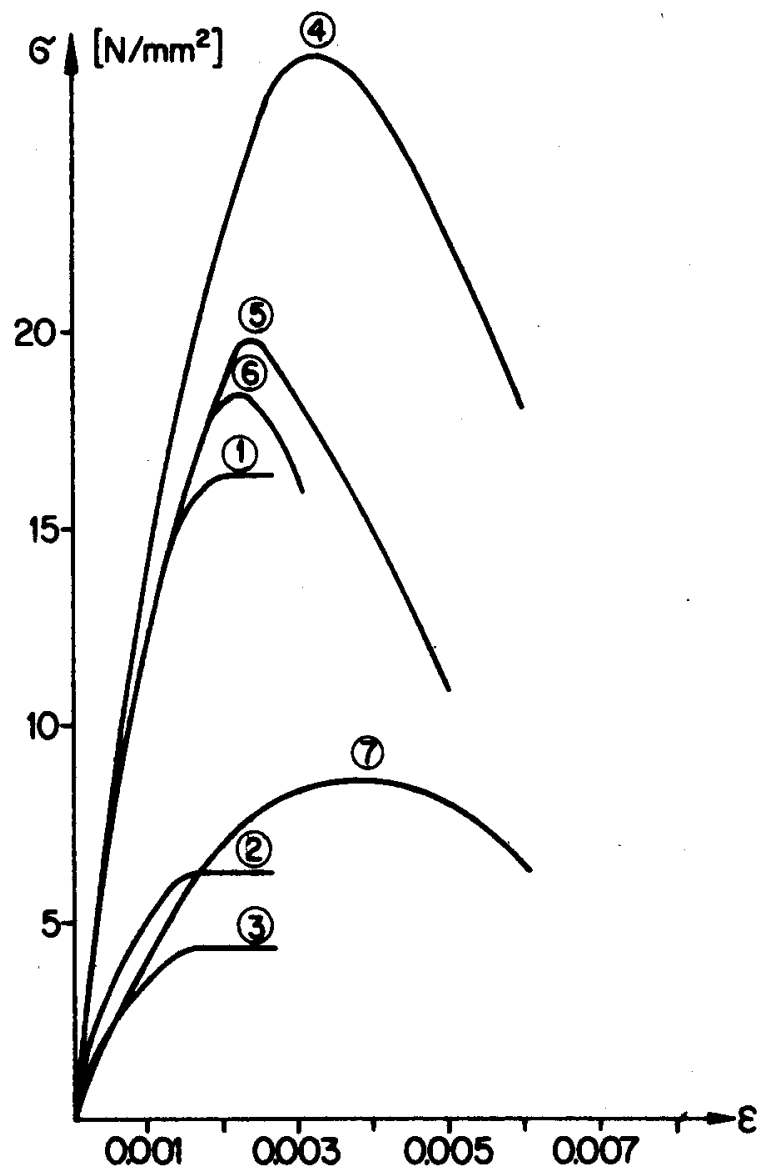

\section{Bild 3.4: Spannungs-Stauchungs-Kurven für} Backsteinmauerwerk

- Die Kurven von Haller [13] wurden an Versuchswänden mit einer Schlankheit $\delta=h / d$ von 20 ermittelt. Die Versuchswände bestanden aus Backsteinen der Qualität $B N\left(B_{S}=28.9 \mathrm{~N} / \mathrm{mm}^{2}\right)$ und verschiedenen Mörteln:

Kurve 1: Zementmörtel P 300

Kurve 2: Verlängerter Mörtel HK $250+P 100$

Kurve 3: Hydraulischer Kalkmörtel HK 350

Unterschiede sind in den E-Moduli sowie den Spannungsmaxima $\sigma_{u}$ erkennbar. Letztere wurden bei Stauchungen $\varepsilon$ von 0.0015 bis 0.0018 erreicht. Die im Anschluss an die Spannungsmaxima horizontal verlaufenden Kurven enden bei Stauchungen $\varepsilon_{u}$ von ca. 0.0026. Es ist jedoch nicht klar ersichtlich, ob es sich dabei tatsächlich um Bruchstauchungen $\varepsilon_{u}$ handelt.

- P. Powell und H.R. Hodgkinson [26] führten Spannungs-Stauchungs-Untersuchungen an acht Stein hohen Pfeilern (h/d $=3$ ) durch. Die insgesamt 26 Versuchskörper wurden aus einem Mörtel ( $\beta_{M}=15.2$ bis $16.1 \mathrm{~N} / \mathrm{mm}^{2}$ ) und vier verschiedenen Backsteinen erstellt:

Kurve 4: $\beta_{S}=71.7 \mathrm{~N} / \mathrm{mm}^{2}$

Kurve $5: \beta_{S}=45.3 \mathrm{~N} / \mathrm{mm}^{2}$

Kurve 6: $\beta_{S}=69.6 \mathrm{~N} / \mathrm{mm}^{2}$

Kurve 7: $\beta_{S}=25.5 \mathrm{~N} / \mathrm{mm}^{2}$ 
Wie im einachsigen Druckversuch des Betons beobachteten sie nach dem Spannungsgipfel einen abfallenden Kurvenast (Strain Softening). Die Spannungsmaxime sowie Bruchstauchungen weisen je nach Mauerstein unterschiedliche Werte auf.

\subsubsection{E-Modul von Mauerwerk}

Das Spannungs-Stauchungs-Verhalten eines Materials kann mit dem Tangentenmodul beschrieben werden. Im Spannungsnullpunkt entspricht dem Tangentender Elastizitätsmodul E. Untersuchungen von Glanville und Barnett [11] sowie von Hilsdorf haben gezeigt, dass der E-Modul zur Mauerwerksfestigkeit $\sigma_{u}$ in Beziehung gebracht werden kann (Bild 3.5).

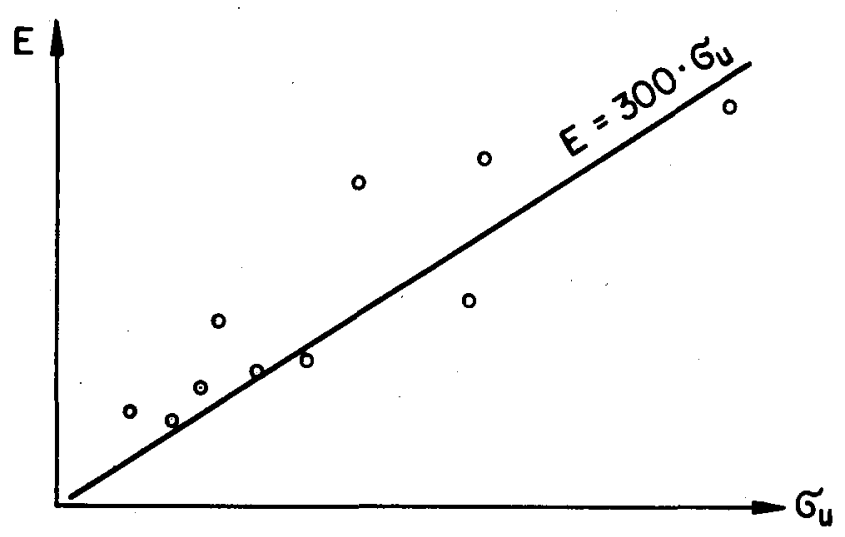

Bild 3.5: $E-\sigma_{u}$

Unter der Annahme eines linear elastischen Stoffgesetzes kann der E-Modul von Mauerwerk bei gegebenen E-Moduli $E_{S}, E_{M}$ der Komponenten Stein und Mörtel sowie bekannten Schichtdicken $h_{S}$, $h_{M}$ folgendermassen angegeben werden. Die Gesamtdeformation $\Delta$ h eines Steins und einer Mörtelschicht unter der Spannung $\sigma$ beträgt:

$\Delta h=\frac{\sigma}{E_{S}} \cdot h_{S}+\frac{\sigma}{E_{M}} \cdot h_{M}$

Diese Gleichung kann umgeschrieben und normiert werden und lautet dann:

$\frac{E}{E_{S}}=\frac{1+\frac{h_{M}}{h_{S}}}{1+\frac{h_{M}}{h_{S}} \cdot \frac{E_{S}}{E_{M}}}$

Für Schichtdickenverhältnisse von $h_{M} / h_{S}=0.05$ und 0.20 wurde $E / E_{S}$ aufgetragen (Bild 3.6). Im Bereich des üblichen Schichtdickenverhältnisses von 0.05 ist der Einfluss des E-Moduls von Mörtel gering. Diese theoretischen Werte wurden durch Untersuchungen von Hilsdorf [17] bei geringen Spannungen bestätigt. Bei grösseren Spannungen weichen die Spannungszustände im Stein und im Mörtel mehr und mehr vom einaxialen Spannungszustand ab. 


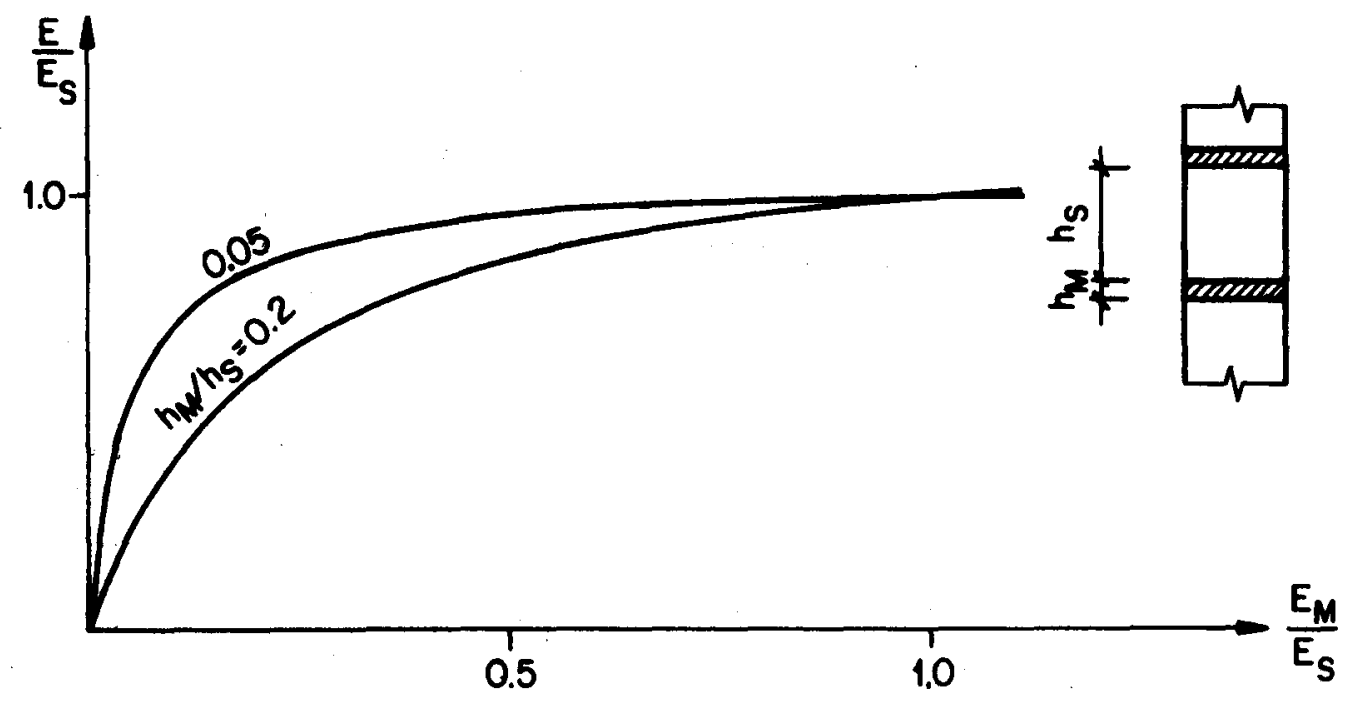

Bild 3.6: E-Modul

\subsubsection{Einaxiale Stein- und Mörteldruckfestigkeit}

Die Mauerwerksfestigkeit $\sigma_{u}$ nimmt bei gleicher Mörtelqualität mit wachsender Steindruckfestigkeit zu. Versuchsergebnisse von Nevander [25] zeigen, dass die Festigkeitszunahme mit wachsender Steindruckfestigkeit $\beta_{S}$ abnehmende Tendenz hat. Untersuchungen von Kirtschig [20] ergaben einen fast linearen Zusammentiang zwischen der Wand- und Steindruckfestigkeit. Dieser Zusammenhang ist in Bild 3.7 a) schematisch dargestellt.

In ähnlicher Weise wird die Mauerwerksfestigkeit $\sigma_{u}$ durch die Mörteldruckfestigkeit $B_{M}$ bei konstanter Steindruckfestigkeit $B_{S}$ beeinflusst. Kreuger [22] hat die Festigkeit von $80 \mathrm{~cm}$ hohen Maverwerkskörpern aus stets demselben Stein und verschiedenen Mörteln untersucht. In Bild 3.7 b) ist der grundsätzliche Verlauf dargestellt. Aufgrund des mehraxialen Spannungszustandes des Mörtels kann $\sigma_{u}$ die axiale Mörteldruckfestigkeit $\beta_{M}$ übersteigen.

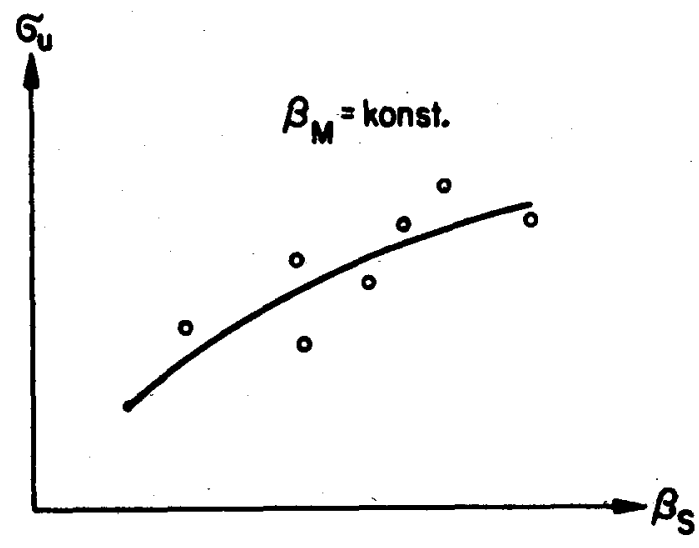

a) $\sigma_{u}-\beta_{8}$

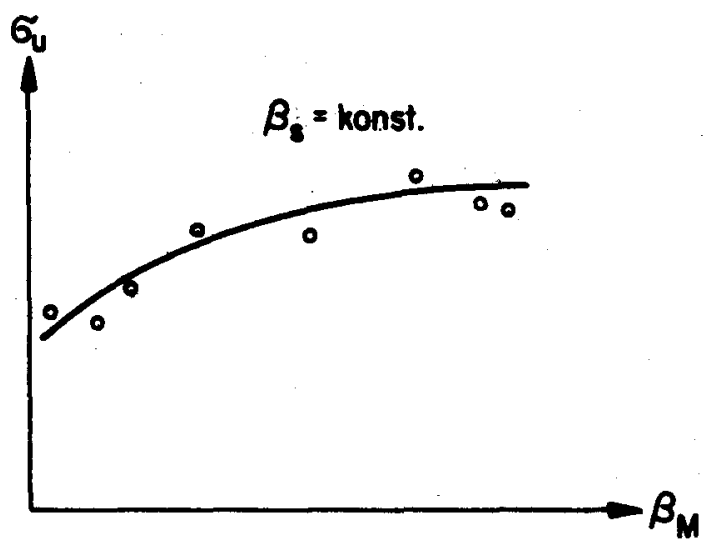

b) $\sigma_{u}-\beta_{M}$

\section{Bild 3.7: $\sigma_{u}$ als Funktion von $\beta_{8}$ und $\beta_{M}$}




\subsubsection{Dicke der Mörtelfuge}

Experimentelle Untersuchungen von Francis, Horman und Jerrems [6] haben gezeigt, dass die Mauerwerksfestigkeit $\sigma_{U}$ mit zunehmender Dicke $h_{M}$ der Mörtelfuge abnimmt (Bild 3.8).

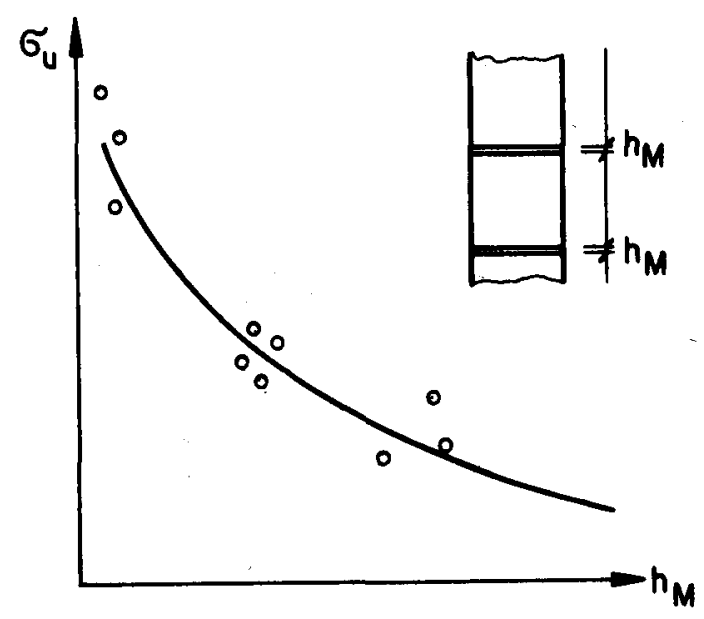

\section{Bild 3.8: $\sigma_{u}$ als Funktion von $h_{M}$}

\subsubsection{Saugfähigkeit des Maversteins}

Die Saugfähigkeit, d.h. die Wassermenge, die ein in eine Wasserfläche gestellter Stein pro Flächen- und Zeiteinheit aufzusaugen vermag, gibt keinen eindeutigen Hinweis bezüglich des Saugvolumens des gleichen Steins, der mit einem Mörtel in Kontakt ist. Untersuchungen von Grandet [12] zeigten, dass einerseits das Saugvolumen des Steins mit zunehmender innerer Oberfläche zunimmt und andererseits mit zunehmender innerer Oberfläche des Mörtels (Wasserrückhaltevermögen) abnimmt. Das resultierende Saugvolumen hängt demzufolge vom Verhältnis zwischen den Kapillaren des Steins und des Märtels ab. Durch die Saugwirkung des Steins erhöht sich zunächst die Haftung zwischen Stein und Mörtel. Bei zunehmendem Wasserentzug verliert jedoch der Mörtel an Erhärtungsvermögen und in frischem Zustand an Verformbarkeit, so dass beim Hin- und Herbewegen der oberen Mauersteine die Mörtelschicht abgewälzt wird (Bild 3.9). Haller [13] bezeichnet diese Erscheinung als Wackeleffekt. In Versuchen an zentrisch und exzentrisch belasteten, geschosshohen wänden hat er gezeigt, dass die Mauerwerksfestigkeit mit zunehmender Saugfähigkeit der Steine abnimmt.

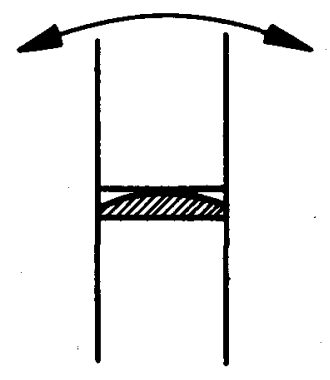

Bild 3.9: Wackeleffekt 
Der Begriff Saugfähigkeit wurde von Haller [15] in den fünfziger Jahren eingeführt. Das Verfahren zur Bestimmung der spezifischen Saugfähigkeit ist in der schweizerischen Mauerwerksnorm [34] seit 1962 geregelt. Die Saugfähigkeit bildet ein wesentliches Kriterium bei der Klassifikation der Mauersteine.

\subsubsection{Zugfestigkeit von Mauerwerk}

Satti und Hendry [31] führten einfache Biegeversuche bei horizontaler Belastung an kleinen Wandkörpern durch. Die Beanspruchung erfolgte parallel und in einem Winkel von 45 und 90 Grad zu den Lagerfugen. Es zeigte sich, dass die Biegezugfestigkeit von der Beanspruchungsrichtung abhängig ist. Senkrecht zu den Lagerfugen war sie fünf- bis siebenmal und unter 45 Grad zwei- bis dreimal kleiner als die Biegezugfestigkeit parallel zu den Lagerfugen. Die Streuung der Ergebnisse war gross.

Die Biegezugfestigkeit senkrecht zu den Lagerfugen ist einerseits durch die Haftzugfestigkeit der Verbindung Stein-Mörtel und andererseits durch die Stein- und Mörtelzugfestigkeit bestimmt. Die Haftzugfestigkeit ist vom resultierenden Saugvolumen zwischen Stein und Mörtel abhängig. Die physikalischchemischen Mechanismen der Haftung zwischen Stein und Mörtel wurden von Grandet [12] untersucht. Sahlin [30] gibt für die Haftzugfestigkeit in Abhängigkeit der Saugfähigkeit des Mauersteins die in Bild 3.10 dargestellte Beziehung an. Sicher sind für die Haftzugfestigkeit auch die Verarbeitbarkeit des Mörtels, sein Wasserrückhaltevermögen, die Steinlochung u.a. von Bedeutung.

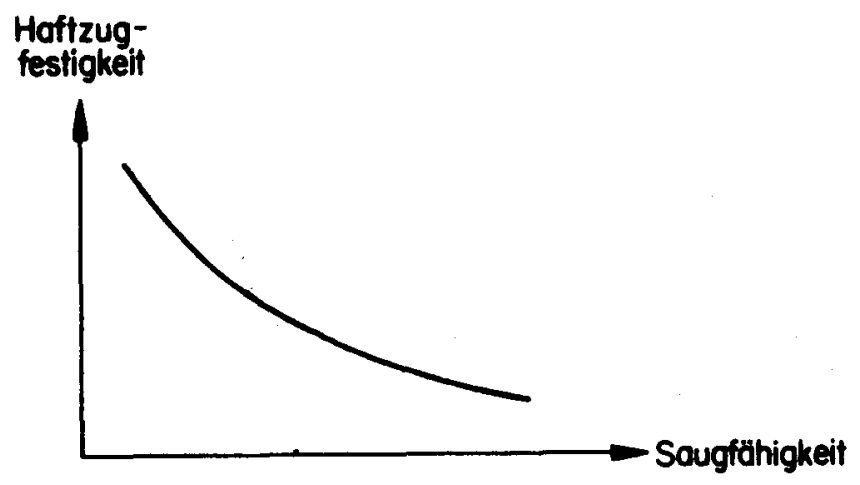

Bild 3.10: Haftzugfestigkeit-Saugfähigkeit 


\section{Momenten / Exzentrizität - Krümmungs-Verhal ten, Interaktionsbeziehung}

\subsection{Ueberblick}

Das Momenten-Krümmungs-Verhalten des Querschnittes ist die Grundlage zur Lösung vieler baustatischer Probleme, bei denen das Kräftespiel durch die Verformungseigenschaften des Materials mitbestimmt wird. Der Berechnung der Momenten-Krümmungs-Beziehungen liegen folgende zwei Annahmen zugrunde:

- Ebenbleiben des Querschnittes

- Stoffgesetz, d.h. Beziehung zwischen der Spannung und Verformung.

Das Ebenbleiben der Querschnitte im Mauerwerk wurde von Haller [13] in Versuchen an $38 \mathrm{~cm}$ dicken Backsteinmauerwerkskörpern bestätigt.

In Kapitel 3 wurde das Stoffgesetz von Mauerwerk dargestellt. Für Mauerwerk können keine einheitlichen Spannungs-Stauchungs-Beziehungen, getrennt nach Mauerwerksgattung (Backsteinmauerwerk, Kalksandsteinmauerwerk u.a.) und Qualität angegeben werden. Im Folgenden werden daher auf der Grundlage von drei einfachen Annahmen für das Stoffgesetz die Momenten- und ExzentrizitätKrümmungs-Beziehungen $(M-\Phi, E-\Phi)$ sowie die Interaktion Moment-Axiallast (M-P) diskutiert. Es handelt sich dabei um folgende drei Stoffgesetze:

- Linear elastisch, ohne Zugfestigkeit

- Linear elastisch, mit beschränkter Zugfestigkeit

- Linear elastisch-ideal plastisch, ohne Zugfestigkeit.

Anstelle der üblicherweise verwendeten M- $\Phi-K u r v e n$ werden in den folgenden Untersuchungen hauptsächlich e- $\Phi$-Kurven diskutiert. Bei einem Material ohne Zugfestigkeit vermag ein Querschnitt kein axiallastunabhängiges Moment aufzunehmen. Ein Moment ergibt sich demnach nur bei exzentrischer Lage der Axiallast. Ist diese entlang einer $M-\Phi-K u r v e$ konstant, kann anstelle von $M$ auch die Exzentrizität e betrachtet werden.

Der Einfluss der Lochung (Grösse, Form und Anordnung der Löcher) der Mauersteine auf das Krümmungs-Verhalten und auf die Interaktion M-P wird mit zwei extremen Annahmen für die Lochung abgeklärt.

Sämtliche Diagramme in diesem Kapitel sind in normierter form dargestellt. Die verwendeten Bezugsgrössen für $P, M_{b}$ und $\Phi_{b}$ basieren dabei auf den folgenden Ansätzen: 


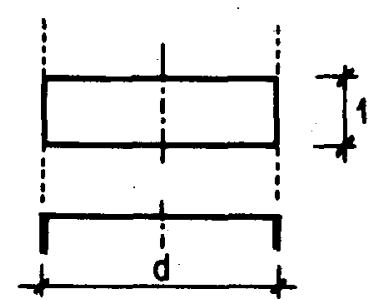

$P_{b}: \quad$ 盂盂血盂盂 $\sigma_{b}$

$M_{b}:$ 血边边 $\sigma_{b}$

$\Phi_{\mathrm{b}}$ :

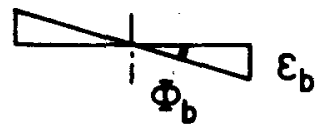

$P_{b}=\sigma_{b} \cdot d$

$M_{b}=\sigma_{b} \cdot \frac{d^{2}}{6}$

$\Phi_{b}=\frac{2 \cdot \varepsilon_{b}}{d}$

$\sigma_{b}$ bzw. $\varepsilon_{b}$ stellt eine Bezugsspannung bzw. eine Bezugsstauchung dar und könnte beispielsweise die Mauerwerksfestigkeit $\sigma_{u}\left(\sigma_{b}=\sigma_{u}\right)$ bzw. die Bruchstauchung $\varepsilon_{u}\left(\varepsilon_{b}=\varepsilon_{u}\right)$ charakterisieren.

\subsection{Linear elastisches Material ohne Zugfestigkeit}

In Bild 3.4 wurde eine Auswahl verschiedener Stoffgesetze für Backsteinmauerwerk dargestellt. Der ansteigende Ast der Spannungs-Stauchungs-Kurven kann vereinfacht mit einer Geraden angenähert werden. Es handelt sich dann um ein linear elastisches Material ohne Zugfestigkeit. Sein Stoffgesetz gehorcht der Gleichung:

$0 \leq \sigma=\varepsilon \cdot E$

und ist in Bild 4.1 in normierter form dargestellt.

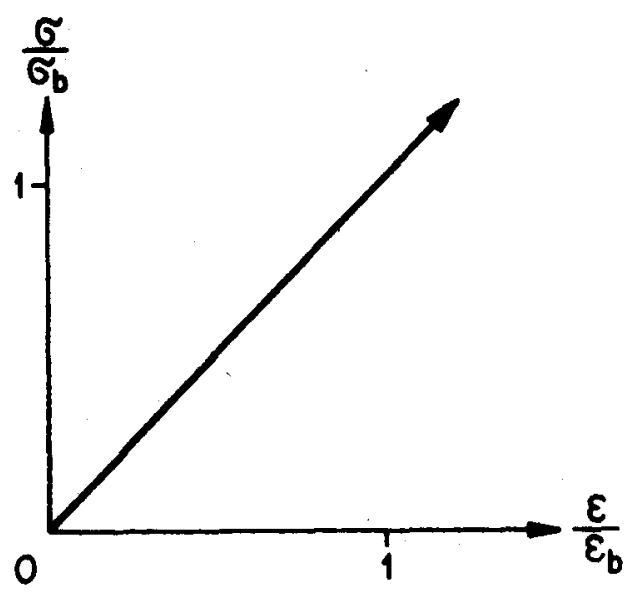

Bild 4.1 : Linear elastisches Material 
Der betrachtete Vollquerschnitt der Breite 1 und Dicke d in Bild 4.2 sei durch eine axiale, bezüglich der Mittelaxe exzentrisch liegende Linienlast $P$ beansprucht. Die Dehnungen bzw. Stauchungen sind voraussetzungsgemäss linear über den Querschnitt verteilt, und die einzelnen Querschnittsfasern können keine Zugkräfte übernehmen. Die Krümmung $\Phi$ ist durch die Neigung der Dehnungsebene zur Querschnittsnormalen gegeben. Das Moment M wird auf die Querschnittsmittelaxe bezogen und lautet:

$M=P \cdot e$
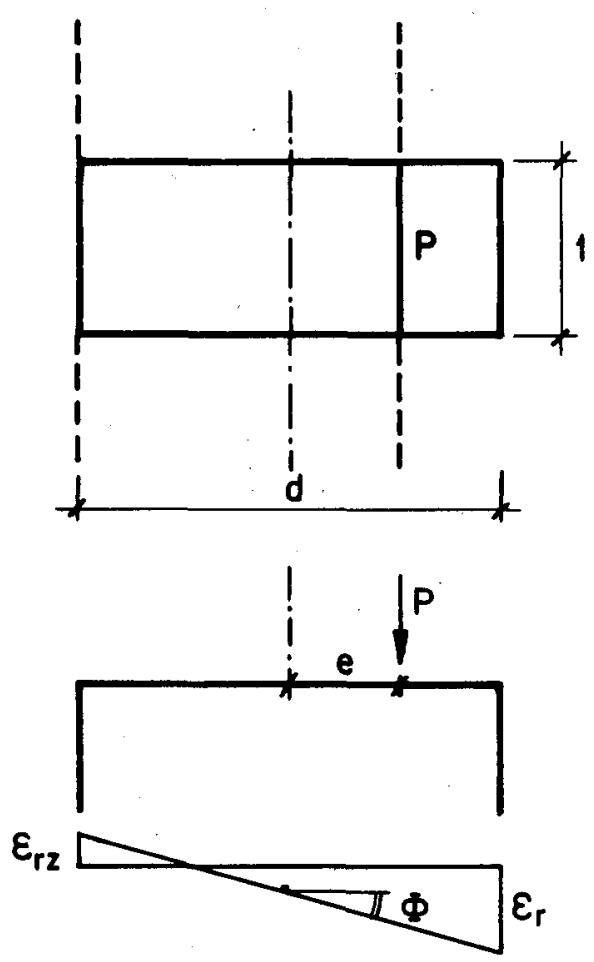

Bild 4.2: Vollquerschnitt, Linienlast, Dehnungsebene

Liegt die Neutralaxe ausserhalb des Querschnittes (Bild 4.3), so erfährt jede Querschnittsfaser eine Stauchung. Die Krümmung $\Phi$ kann durch M. wie folgt ausgedrückt werden:

$\Phi=\frac{M}{\left(\frac{d^{2}}{6}\right)} \cdot \frac{2}{E \cdot d}$

Durch Division der GI. (4.4) mit $\sigma_{b}$ und nach Einführung der Bezugsgrössen $\Phi_{b}$ und $M_{b}$ ergibt sich:

$\frac{M}{M_{b}}=\frac{\Phi}{\Phi_{b}}$

Wird anstelle von M die Exzentrizität e verwendet, so erhält man aus Gl. (4.4) und nach Einführung von $\sigma_{b}, P_{b}$ und $\Phi_{b}$ :

$\frac{e}{d}=\frac{1}{6} \cdot \frac{\Phi / \Phi_{b}}{P / P_{b}}$ 


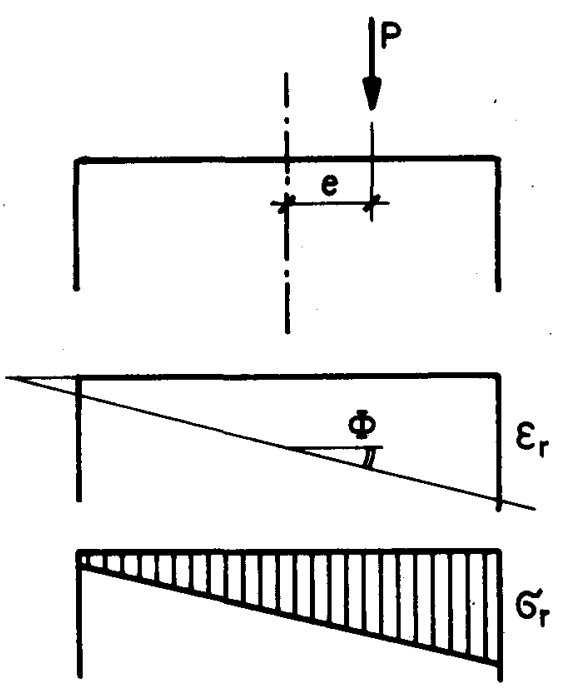

Bild 4.3 : Neutralaxe ausserhalb Querschnitt

Die Beziehungen (4.4) bis (4.6) sind gültig, sofern:

$\Phi \cdot d \leq \varepsilon_{r}$

das heisst:

$\frac{M}{M_{b}} \leq \frac{P}{P_{b}}$

$\frac{e}{d} \leq \frac{1}{6}$

Die Randspannung $\sigma_{r}$ kann durch $P$ und $\Phi$ wie folgt ausgedrückt werden:

$\sigma_{r}=\frac{P}{d}+\frac{\Phi \cdot d}{2} \cdot E$

Dividiert man wiederum die ganze Gleichung durch $\sigma_{b}$ und führt $P_{b} b z w$. $\Phi_{b}$ ein, so erhält man:

$\frac{\Phi}{\Phi_{b}}=\frac{\sigma_{r}}{\sigma_{b}}-\frac{P}{P_{b}}$

Mit grösser werdender Exzentrizität e wandert die Neutralaxe gegen die Wandaxe zu und kommt beim Ueberschreiten der Gültigkeitsgrenzen von Gl. (4.8) ins Innere des Querschnittes zu liegen (Bild 4.4). Die gedehnten Querschnittsfasern vermögen keine Zugkräfte aufzunehmen. Der wirksame Querschnitt vermindert sich daher.

Für $P$ bzw. e können folgende Beziehungen angeschrieben werden:

$P=\frac{\sigma_{r}^{2}}{2 \cdot \Phi \cdot E}$ 
$e=\frac{d}{2}-\frac{\sigma_{r}}{3 \cdot E \cdot \Phi}$

Bestimmt man aus Gl. (4.11) $\sigma_{r}$ und setzt diesen Wert in GI. (4.12) ein, so erhält man:

$e=\frac{d}{2}-\sqrt{\frac{2 \cdot P}{g \cdot E \cdot \Phi}}$

und nach Division durch d und Einführung der Bezugsgrössen $P, M_{b}$ und $\Phi_{b}$ ergibt sich:

$\frac{M}{M_{b}}=3 \cdot \frac{P}{P_{b}} \cdot\left(1-\sqrt{\frac{4 \cdot P \cdot \Phi}{9 \cdot P_{b} \cdot \Phi}}\right)$

$\frac{e}{d}=\frac{1}{2} \cdot\left(1-\sqrt{\frac{4 \cdot \bar{P} \cdot \Phi_{b}}{g \cdot P_{b} \cdot \Phi}}\right)$

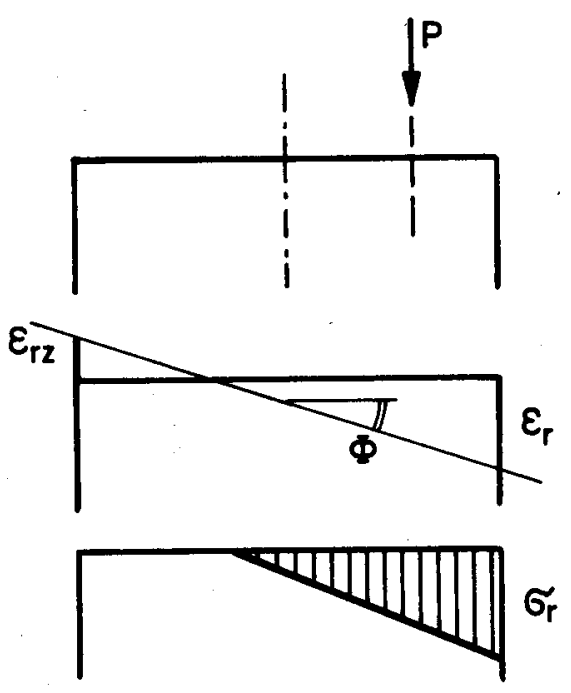

Bild 4.4: Neutralaxe innerhalb Querschnitt

Aus 61. (4.11) kann durch Division mit $\sigma_{b} \cdot d$ und Einführung von $\Phi_{b}$ und $P_{b}$ der folgende Zusammenhang erhalten werden:

$\frac{P}{P_{b}}=\frac{\left(\frac{\sigma_{r}}{\sigma_{b}}\right)^{2}}{4 \cdot \frac{\Phi}{\Phi_{b}}}$

Setzt man den Ausdruck für $P / P_{b}$ von Gl. (4.16) in Gl. (4.15) ein, ergibt sich:

$\frac{e}{d}=\frac{1}{2}-\frac{1}{6} \cdot \frac{\frac{\sigma_{r}}{\sigma_{b}}}{\frac{\Phi}{\Phi_{b}}}$ 
Zwischen $\varepsilon_{\mathrm{rz}}$, $\sigma_{\mathrm{r}}$ und $\Phi$ besteht der Zusammenhang

$\varepsilon_{r z}=\frac{\sigma_{r}}{E}-\Phi \cdot d$

Ermittelt man aus Gl. (4.18) $\sigma_{r}$ und führt die Bezugswerte $\Phi_{b}$, $\sigma_{b}$ und $\varepsilon_{b}$ ein, erhält man durch Einsetzen in Gl. (4.17)

$\frac{e}{d}=\frac{1}{\sigma} \cdot\left(1-\frac{\frac{\varepsilon_{r z}}{\varepsilon_{b}}}{\frac{\Phi}{\Phi_{b}}}\right)$

\subsubsection{M- $\Phi-$ und $e-\Phi-K u r v e n$}

In Bild 4.5 sind die $M-\Phi-K u r v e n$ der Gleichungen (4.5) und (4.14) dargestellt. Zusätzlich ist die Kurve konstanter Randspannung $\sigma_{\Gamma}\left(\sigma_{\Gamma}=\sigma_{b}\right)$ fein gestrichelt und diejenige gleicher Ranidehnung $\varepsilon_{r z}\left(\varepsilon_{r z}=-0.5 \cdot \varepsilon_{b}\right)$ strichpunktiert eingezeichnet.

Den stark ausgezogenen $M-\Phi-K u r v e n$ entlang hat die auf $P_{b}$ bezogene Axiallast $P$ einen jeweils konstanten Wert $\left(P / P_{b}=0.25\right.$ und 0.50$)$. Eine Vergrösserung von $M$ ergibt sich demnach nur durch eine Vergrösserung der Exzentrizität e. Sofern e die Kernweite $d / 6$ nicht überschreitet, folgen diese M- $\Phi-K u r v e n$ der

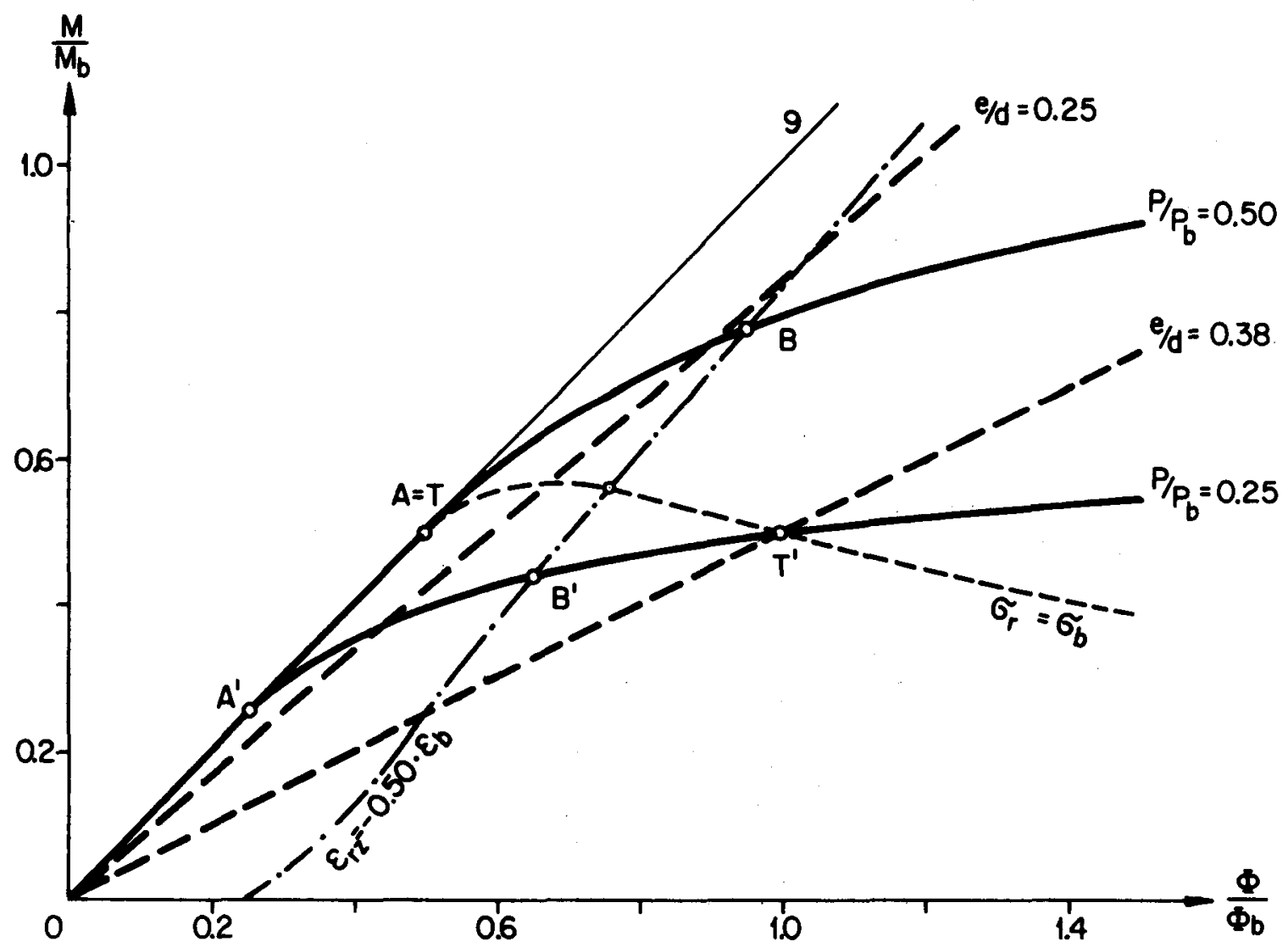

Bild 4.5: $M-\Phi-$ Kurven 
Geraden g mit der Gl. (4.5). In den Punkten A und A' liegt die Axiallast am Kernrand. Bei grösseren Exzentrizitäten werden einzelne Querschnittsfasern gedehnt. Die Krümmungen nehmen dann überproportional zum Moment zu, und die M- $\Phi-K u r v e n$ schneiden die Kurve konstanter Randdehnung $\varepsilon_{r z}$ (strichpunktiert) in $B$ und $B^{\prime}$. Die Kurve konstanter Randspannung $\sigma_{\Gamma}$ (fein gestrichelt) wird in $T$ und $T$ ' geschnitten. Die Punkte $A$ und $T$ fallen zusammen, d.h. in $A$ bzw. $T$ liegt die Axiallast am Kernrand und die Randspannung $\sigma_{r}$ beträgt $\sigma_{b}$. Bei kleinerer Axiallast $\left(P / P_{b}=0.25\right)$ wird diese Randspannung erst bei wesentlich grösserer Krümmung (Punkt $T^{\prime}$ ) erreicht. Demgegenüber nehmen die Krümmungen für konstante Randdehnung $\varepsilon_{\Gamma z}\left(B^{\prime}\right.$ und $B$ ) mit wachsender Axiallast zu. Dieser Sachverhalt ist in Bild 4.6 detailliert dargestellt. Den gestrichelten Kurven entlang ist die Exzentrizität konstant (e/d $=0.25,0.33)$. Das Moment M nimmt demnach proportional zur Axiallast $P$ zu. Sofern e nicht grösser als die Kernweite $d / 6$ ist, folgt die zugehörige M- $\Phi-K u r v e$ der Geraden $g$ mit der Gl. (4.5). Bei grösseren Exzentrizitäten verlaufen die M- $\Phi$-Kurven ebenfalls linear, die Steifigkeit bzw. die Neigung der Geraden wird jedoch aufgrund des verminderten Querschnittes geringer.

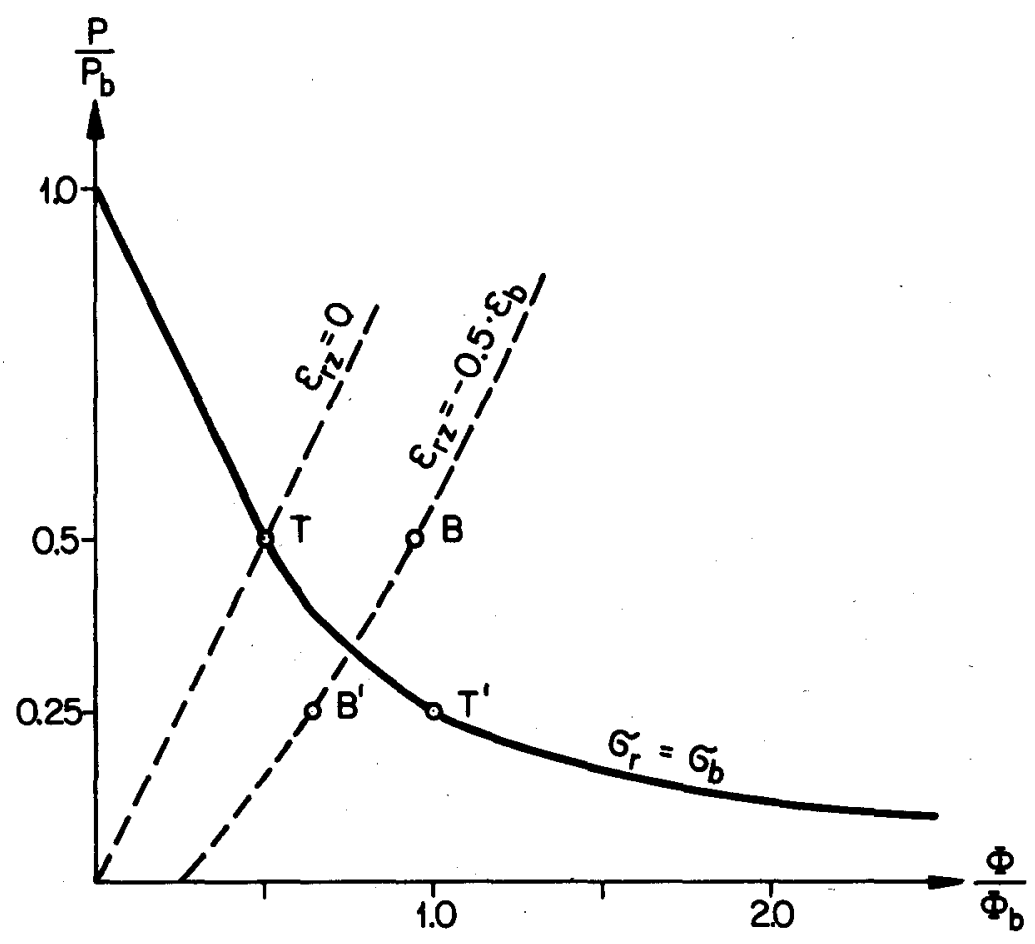

Bild 4.6: $P-\Phi$

In Bild 4.7 sind die e- $\Phi-K u r v e n$ dargestellt. Die Axiallast $P$ ist den stark ausgezogenen Kurven entlang konstant $\left(P / P_{b}=0.01,0.10,0.25\right.$ und 0.50$)$. Die zwei übereinander liegenden Geradenabschnitte $O A$ und $O A^{\prime}$ von Bild 4.5 klaffen in dieser Darstellung auseinander. In $A$ und $A$ ' liegt die Axiallast $P$ am Kernrand $(e / d=1 / 6)$. Bei grösseren Exzentrizitäten wachsen die Krümmungen überproportional an. Die Punkte $B, T$ und $B^{\prime}, T^{\prime}$ entsprechen den Punkten in Bild 4.5. 


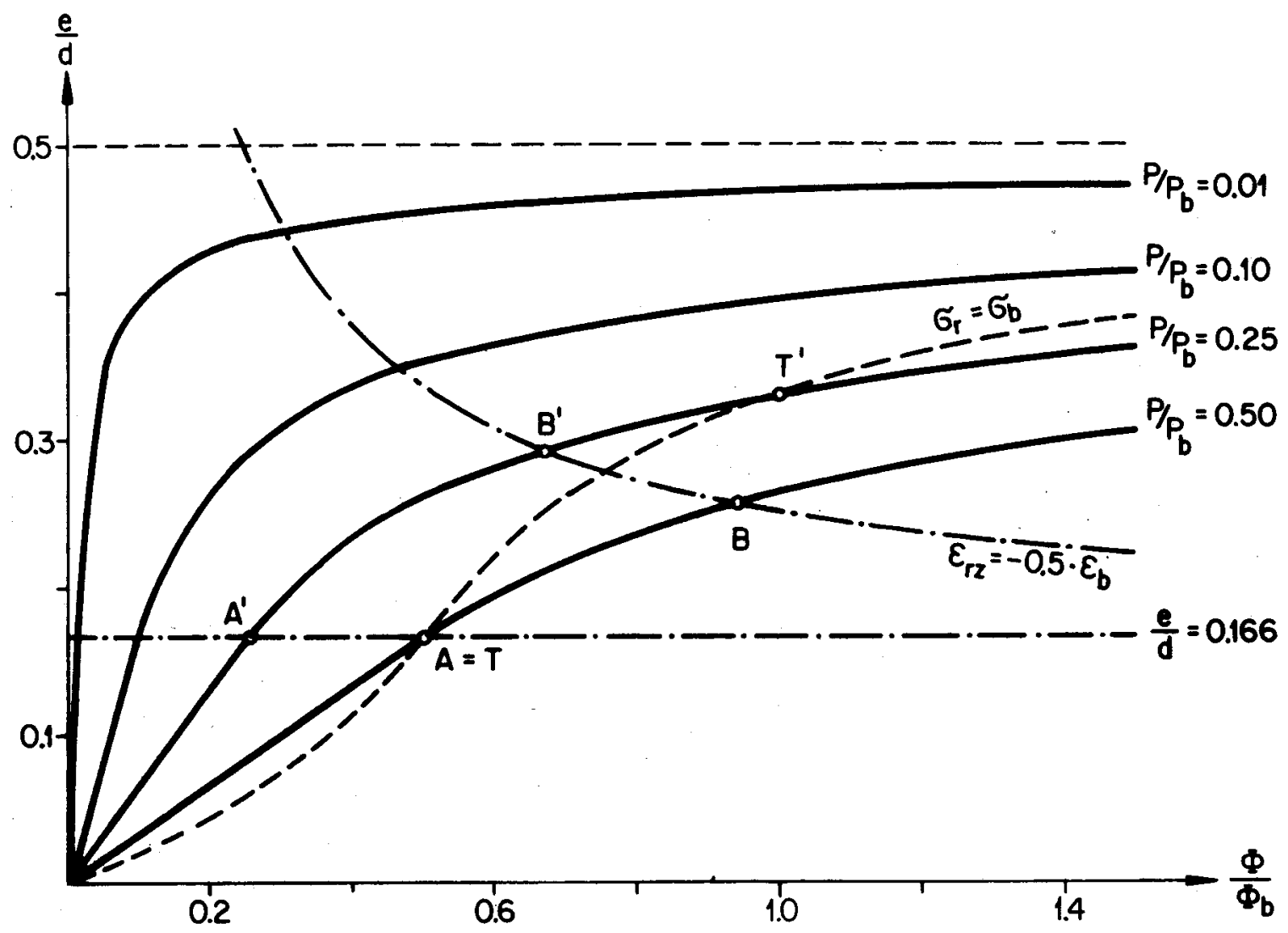

\section{Bild 4.7: e - $\Phi-$ Kurven}

Es zeigt sich, dass bei einem Material ohne Zugfestigkeit die Exzentrizität die halbe Querschnittsdicke d nicht überschreiten kann. Je kleiner die Axiallast, desto mehr schmiegt sich die e- $\Phi-K u r v e$ an die Ordinate bzw. Parallele zur Abszisse im Abstand von 0.5 ( $v g l \cdot$ Kurve für $P / P_{b}=0.01$ ).

\subsubsection{Interaktion $M-P$}

Den stark ausgezogenen Kurven in Bild 4.8 entlang ist die Randspannung $\sigma_{r}$ konstant $\left(\sigma_{r}=\sigma_{b}\right)$. Die Kurven stellen die Interaktionsbeziehungen zwischen Moment und Axiallast sowie den entsprechenden Zusammenhang zwischen der Exzentrizität und der Axiallast dar. Da das vorausgesetzte Material keine Zugfestigkeit besitzt, verläuft die Interaktionskurve durch den Ursprung. Die Exzentrizität beträgt in diesem Punkt $d / 2$.

Das maximale Moment $M_{\max }=9 / 16 \cdot M_{b}$ wird bei einer Exzentrizität e von $d / 4$ erreicht, und die dazugehörige Axiallast $P$ beträgt $3 / 8 \cdot P_{b}$.

Im schaffierten Gebiet treten für alle Kombinationen von M und P keine Dehnungen auf, und die Randspannung $\sigma_{r}$ überschreitet $\sigma_{b}$ nicht. Die Verbindung der Punkte $A, T, B$ bzw. A', T', B' entspricht den M- $\Phi-K u r v e n$ von Bild 4.5 und den e- $\Phi$-Kurven von Bild 4.7 . 


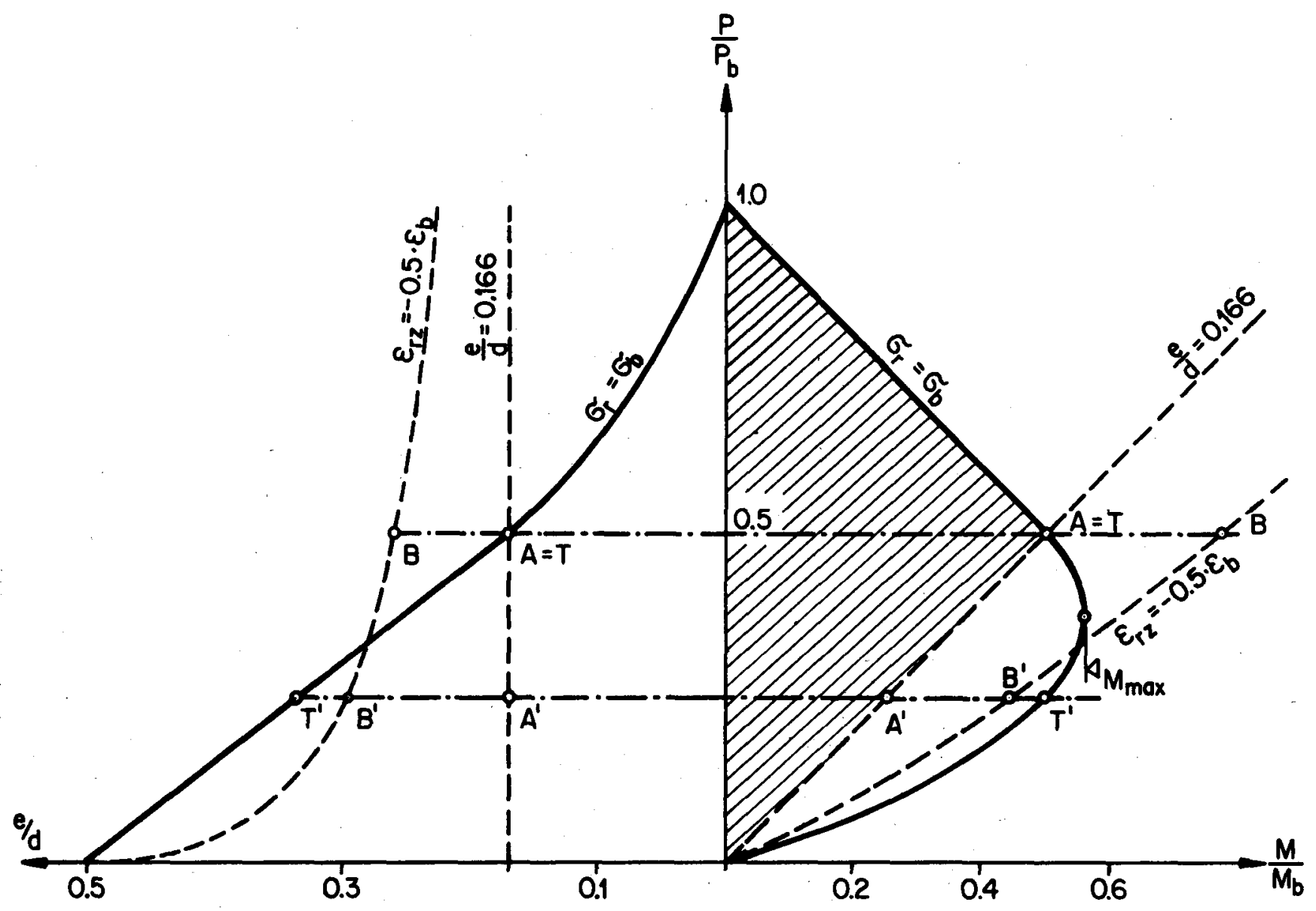

Bild 4.8: Interaktion $M-P$

\subsection{Linear elastisches Material mit beschränkter Zugfestigkeit}

Die Zugfestigkeit $\sigma_{z u}$ von Mauerwerk ist einerseits durch die Haftzugfestigkeit der Verbindung Stein-Mörtel und andererseits durch die Stein- und Mörtelzugfestigkeit bestimmt. Sie ist demzufolge von vielen Einflussgrössen abhängig (vgl.3.3.5). Mit den folgenden Untersuchungen über den Einfluss der Zugfestigkeit im Krümmungs-Verhalten sowie in der Interaktions-Beziehung M-P soll aufgezeigt werden, inwiefern die Zugfestigkeit und insbesondere auch deren genauere Kenntnis von Bedeutung ist.

Vorausgesetzt wird das in Bild 4.9 dargestellte Stoffgesetz. Die Zugfestigkeit $\sigma_{z u}$ wird mit $\zeta$ in Beziehung $z u \sigma_{b}$ gebracht:

$$
\begin{aligned}
& \sigma_{z u}=-\zeta \cdot \sigma_{b} \\
& \varepsilon_{z u}=-\zeta \cdot \varepsilon_{b} \\
& \sigma_{z u} \leq \sigma=E \cdot \varepsilon
\end{aligned}
$$




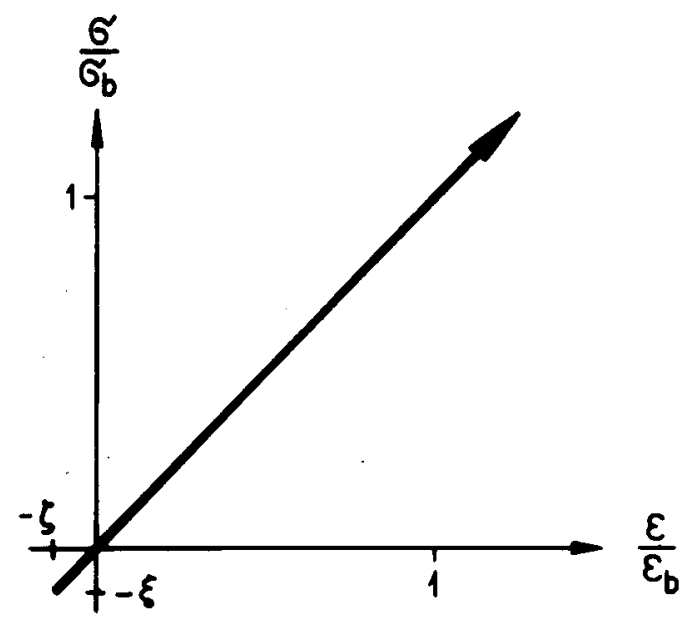

Bild 4.9 : Linear elastisches Material mit Zugfestigkeit

Im Anhang $A 2$ sind die analytischen Beziehungen der e- $\Phi-K u r v e n$ und der Interaktion $M-P$ angegeben.

\section{3 .1 e- $\Phi-$ Kurven}

In Bild 4.10 sind für drei Axiallasten $\left(P / P_{b}=0.10,0.25\right.$ und 0.5$)$ sowie drei $\zeta$-Werte $(\zeta=0.0,0.1$ und 0.2$)$ die $e-\Phi$-Kurven dargestellt. Die Kurven konstanter Randdehnung $\left(\varepsilon_{\mathrm{rz}}=-0.2 \cdot \varepsilon_{b}\right)$. bzw. konstanter Randspannung $\left(\sigma_{r}=\sigma_{b}\right)$ sind ebenfalls für die drei $\zeta$-Werte fein eingetragen.

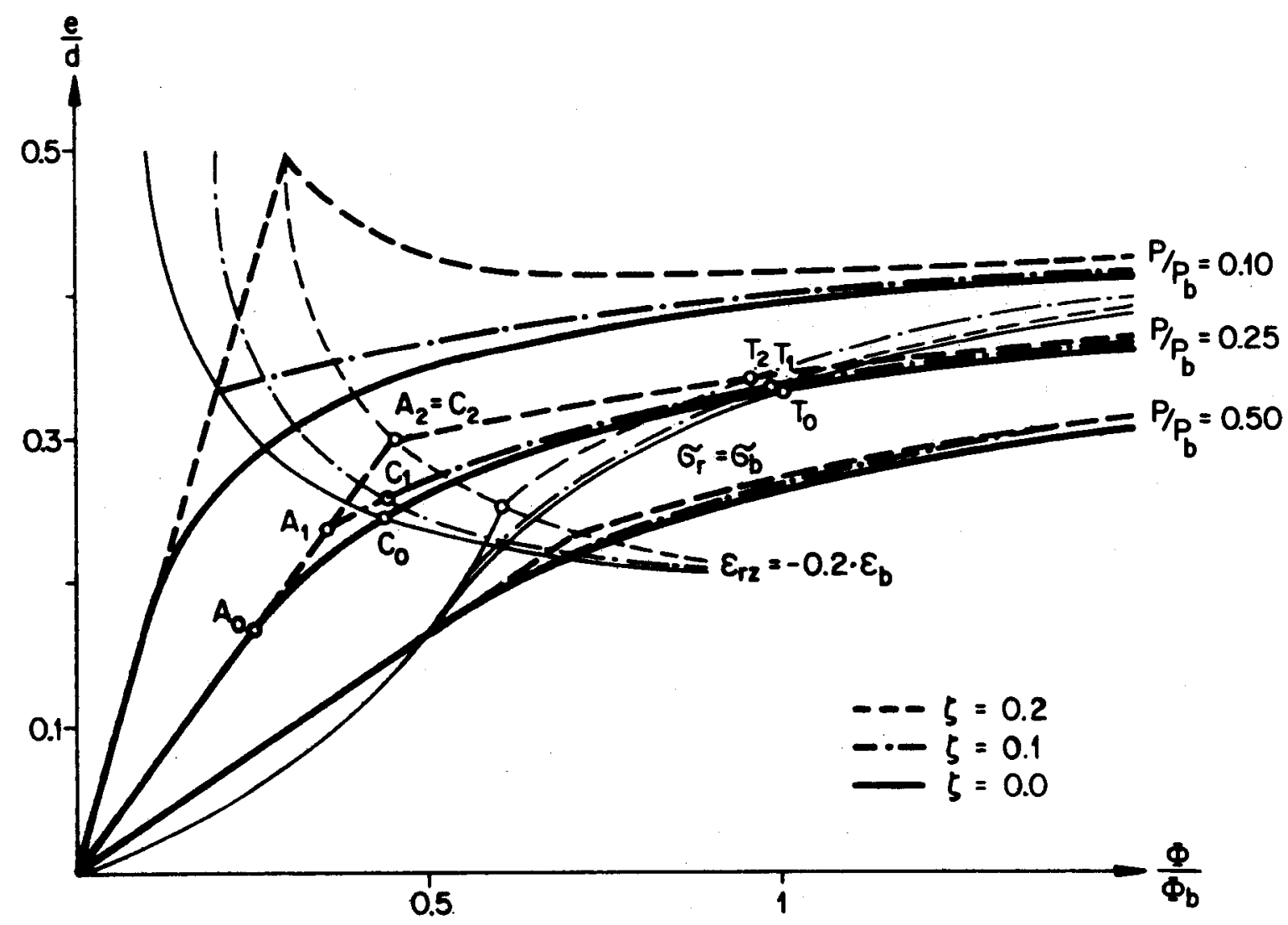

Bild 4.10: e - $\Phi$ - Kurven 
Uebersteigt die Exzentrizität die Kernweite, so ergeben sich in einzelnen Querschnittsfasern Dehnungen. Ohne Zugfestigkeit $(\zeta=0)$ vermindert sich der wirksame Querschnitt und die e- $\Phi-K u r v e n$ flachen ab (Punkt $A_{0}$ in $e-\Phi-K u r v e$ für $\left.P / P_{b}=0.25\right)$. Bei vorhandener Zugfestigkeit ( $\zeta=0.1$ und 0.2$)$ wirken die gedehnten Fasern bis zur Zugfestigkeit mit. Dementsprechend verlaufen die e- $\Phi-$ Kurven bis zu diesen Punkten $A_{1}, A_{2}$ linear. Nach dem Ueberschreiten der Zugfestigkeit ergibt sich in den e- $\Phi-K u r v e n$ ein Knick, der bei grösserer Zugfestigkeit bzw. Kleinerer Axiallast (vgl. $\left.P / P_{b}=0.10\right)$ deutlicher in Erscheinung tritt.

Bei grösseren Krümmungen schmiegen sich die e- $\Phi-K u r v e n$ an diejenigen mit $\zeta=0$ an.

Ohne Zugfestigkeit vermögen gedehnte Querschnittsfasern keine Zugkräfte aufzunehmen. In $A_{0}$ bildet sich demzufolge zwischen den beiden Schnittufern eine klaffende Fuge, deren spezifische Deffnung der Randdehnung $\varepsilon_{r z}$ entspricht. In $c_{0}$ beträgt $\varepsilon_{r z}=-0.2 \cdot \varepsilon_{b}$. Bei einem Material mit Zugfestigkeit bildet sich eine solche Fuge erst nach dem Ueberschreiten der Zugfestigkeit (Punkte $A_{1}$ und $A_{2}$ ). Die spezifische Deffnung entspricht dann der Bruchdehnung $\varepsilon_{z u}$. Die Krümmung, bei der sich die gleiche spezifische Deffnung bildet, nimmt mit wachsender Zugfestigkeit nur gering $z u$ (vgl. $\Phi$ in den Punkten $C_{0}, C_{1} u n d C_{2}$ ). Demgegenüber ergibt sich bei konstanter Axiallast (z.B. $\left.P / P_{b}=0.25\right)$ die Randspannung $\sigma_{r}=\sigma_{b}$ mit wachsender Zugfestigkeit bei kleinerer Krümmung (vgl. Punkte $T_{0}, T_{1}$ und $T_{2}$ ). Allerdings sind diese Einflüsse der Zugfestigkeit sehr gering. Diese Zusammenhänge sind in Bild 4.11 deutlicher dargestellt.

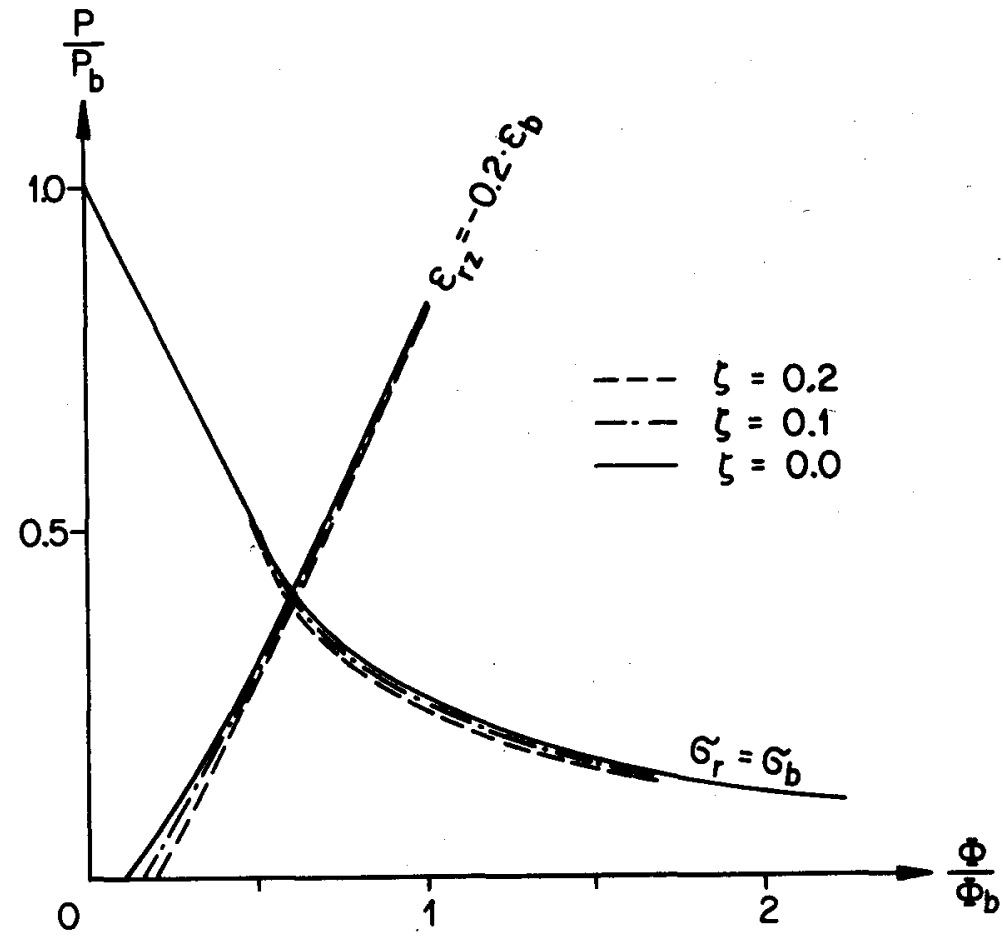

Bild 4.11: $P-\Phi$ 


\subsubsection{Interaktion M-P}

In Bild 4.12 sind die Interaktionskurven $M-P$ und die dazugehörigen Verläufe der Exzentrizität für drei verschiedene $\zeta$-Werte $(\zeta=0.0,0.1$ und 0.2$)$ dargestellt. In jedem Punkt der Kurven ist die Randdruckspannung $\sigma_{r}$ gleich der Bezugsspannung $\sigma_{b}$ oder die Randzugspannung $\sigma_{r z}$ gleich der Zugfestigkeit $\sigma_{z u}$.

Bei grosser Axiallast $\left(P \geq 0.5 \cdot P_{b}\right)$ ist der ganze Querschnitt überdrückt. Die Zugfestigkeit beeinflusst daher in diesem Lastbereich die Interaktion M-P bzw. die e-P-Kurve nicht. Im unteren Lastbereich $\left(P<0.5 \cdot P_{b}\right)$ werden die Momente $M$ bei gleicher Axiallast $P$ mit zunehmender Zugfestigkeit grösser. Im Momentenmaximum (Punkt $W$ ) ist $\sigma_{r}=\sigma_{b}$ und $\sigma_{r z}=\sigma_{z u}=-\zeta \cdot \sigma_{b}$. Nach dem Ueberschreiten von $\sigma_{z u}$ ergibt sich in der M-P-Kurve ein Knick. Den Abszissenwerten (Punkte $R_{1}, R_{2}$ ) entsprechen die Rissmomente $M / M_{b}=\zeta$.

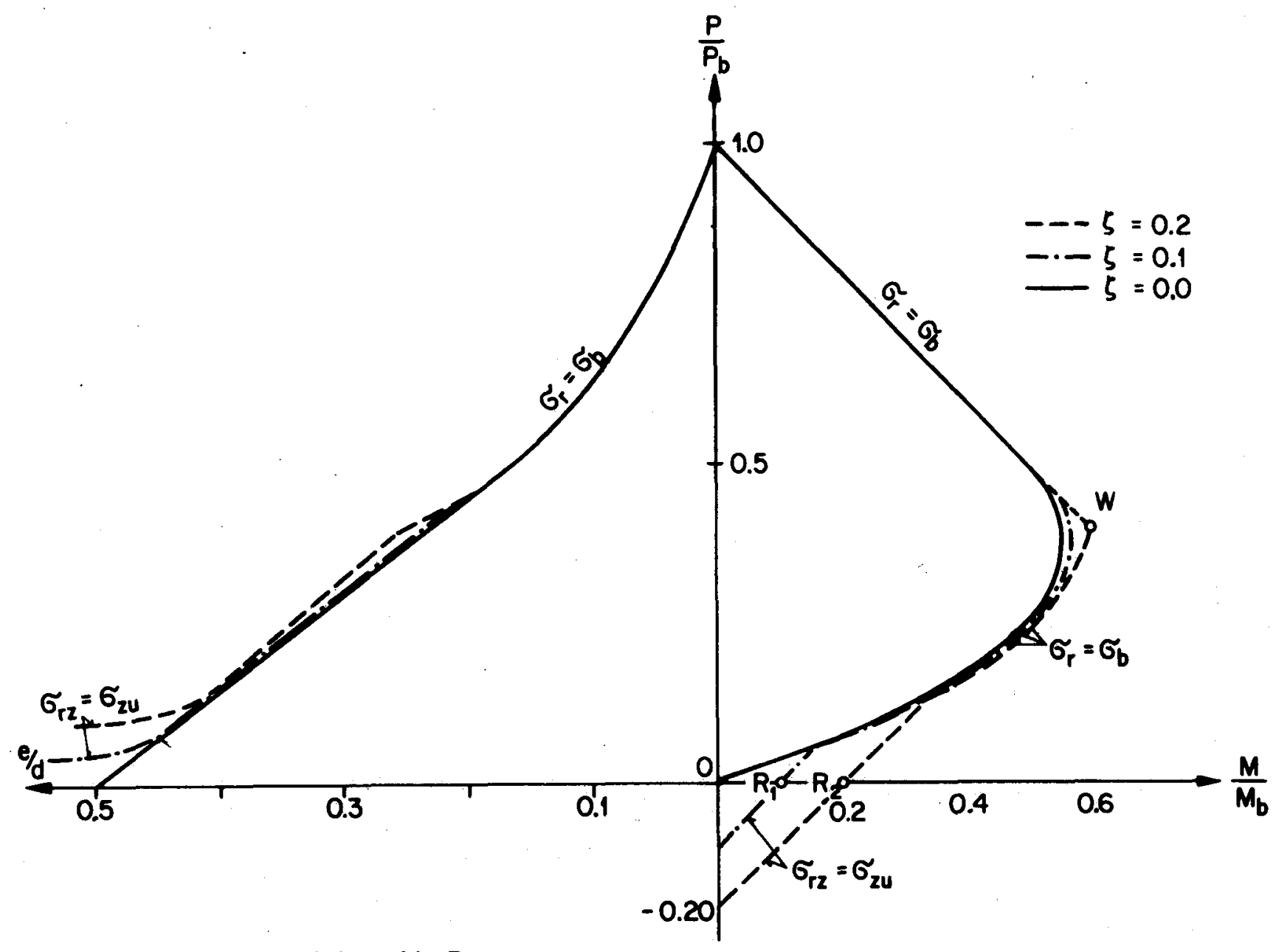

Bild 4.12: Interaktion $M-P$ 
4.4 Linear elastisch-ideal plastisches Material ohne Zugfestigkeit

Die in Bild 3.4 dargestellten Spannungs-Stauchungs-Kurven weisen teilweise ausgeprägte Spannungsgipfel oder Fliessplateaus auf. Die folgende Untersuchung soll den Einfluss eines derartigen Materialverhaltens genauer abklären.

Vorausgesetzt sein ein linear elastisch-ideal plastisches Material ohne Zugfestigkeit:

$\varepsilon<0: \sigma=0$

$0 \leq \varepsilon \leq \varepsilon_{b}: \sigma=\varepsilon \cdot E$

$\varepsilon \leq \varepsilon \quad: \quad \sigma=\sigma_{b}$

Die plastische Stauchung sein unbeschränkt. Das Stoffgesetz ist in Bild 4.13 in normierter form dargestellt.

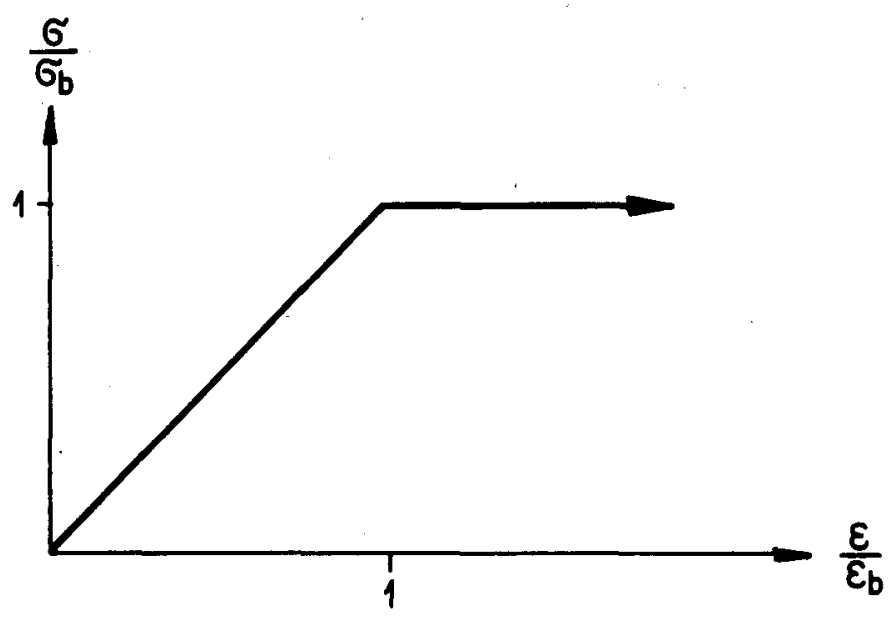

Bild 4.13: Linear elastisch-ideal plastisches Material

Im Anhang A3 sind die analytischen Beziehungen der e- $\Phi-K u r v e n$ und der Interaktion $M-P$ angegeben. 


\section{4 .1 e- $\Phi$-Kurven}

Bis zu Randspannungen $\sigma_{r}=\sigma_{b}$ bzw. Randstauchungen $\varepsilon_{r}=\varepsilon_{b}$ ergeben sich gegenüber den fett gestrichelten $8-\Phi-K u r v e n$ des linear elastischen Materials (vgl. auch Bild 4.7) keine Abweichungen (Bild 4.14). Bei grösseren Randstauchungen $\left(\varepsilon_{\Gamma}>\varepsilon_{b}\right)$ flachen die e- $\Phi$-Kurven (stark ausgezogen) hingegen stärker $a b, d . h$. bei gleicher Krümmung wird die Exzentrizität kleiner. Dieser Sachverhalt verdeutlicht sich mit zunehmender Axiallast (vgl. Kurven $P / P_{b}=0.25$ und 0.50 ).

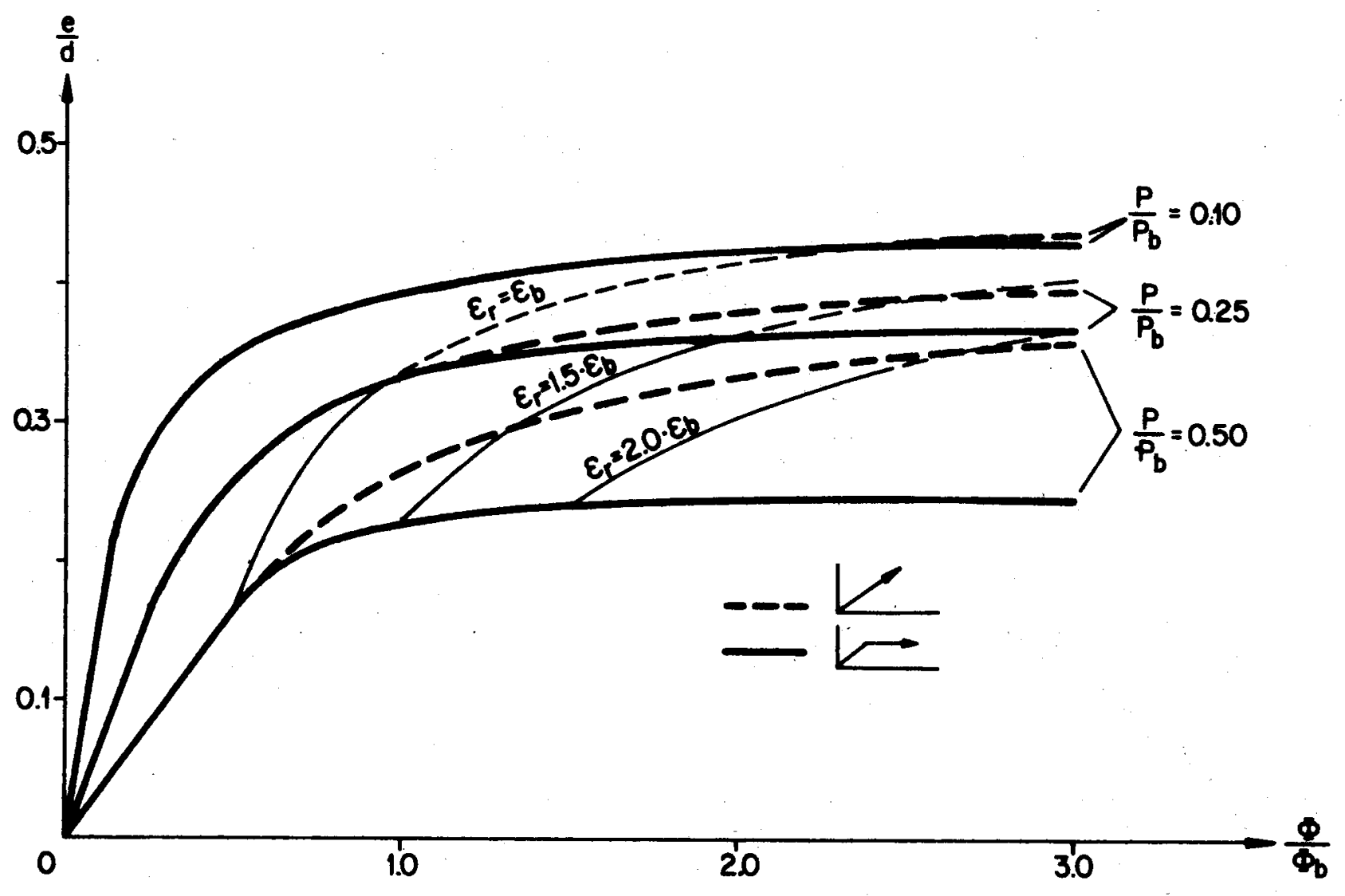

Bild 4.14: e - $\Phi$-Kurven 


\subsubsection{Interaktion M-P}

In Bild 4.15 sind für verschiedene Werte von $\varepsilon_{r} / \varepsilon_{b}$ die Interaktionskurven und die dazugehörigen Kurven der Beziehungen zwischen der Exzentrizität und der Axiallast dargestellt. Im Bereich grösserer Axiallasten ( $\left.P>0.4 \cdot P_{b}\right)$ werden die Momente durch Plastifizierung markant vergrössert, entsprechend gross ist in diesem Lastbereich auch die Zunahme der Exzentrizität.

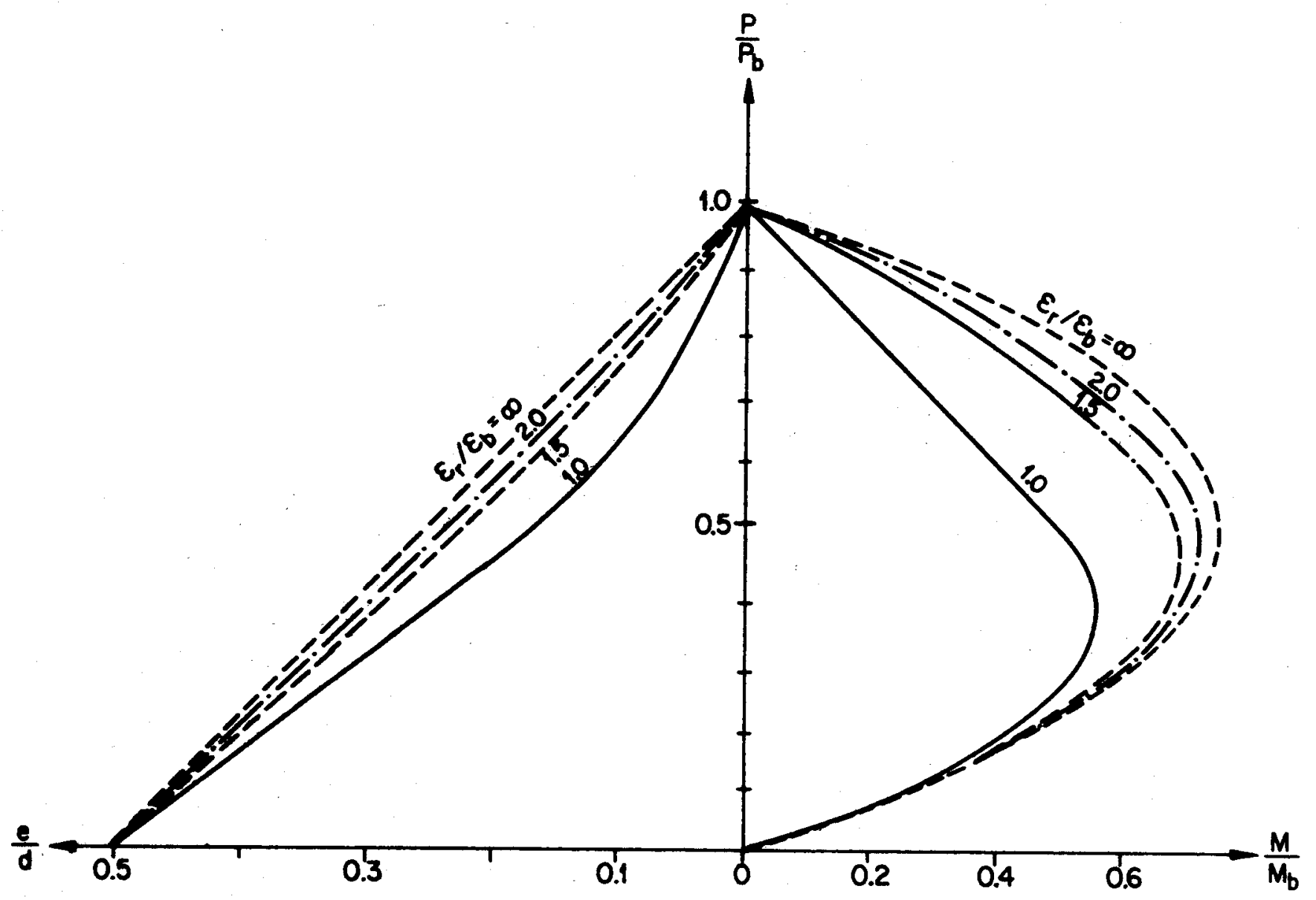

Bild 4.15: Interaktion $M-P$ 
In Kapitel 3 wurde auf die Vielfalt von Mauersteinen hinsichtlich des Materials, der technischen Eigenschaften, des formates und der Lochung hingewiesen. In den letzten Jahren standen bei der Herstellung der Mauersteine in der Regel wärmetechnische Aspekte im Vordergrund. Zur Erzielung einer besseren Wärmedämmung wurde insbesondere beim Backstein der prozentuale Lochflächenanteil Lo erhöht. Die Lochung, d.h. die Form, Grösse und Anordnung der Löcher wurde verändert [27]. Im Kalksandstein blieb der Lochflächenanteil geringer, da bei diesem Stein weniger wärmedämmende Aspekte im Vordergrund standen. Das Gasbetonstein wird aufgrund seiner geringen Festigkeit nicht gelocht.

Mit den folgenden Untersuchungen soll der Einfluss der Lochung auf das Krümmungs-Verhalten und auf die Interaktion M-P gezeigt werden. Vorausgesetzt werden das linear elastische Material ohne Zugfestigkeit (Bild 4.1) sowie zwei extreme Annahmen für die Lochung (Bild 4.16).

\section{Lochung A}

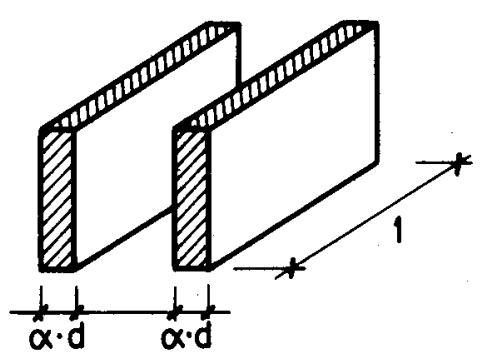

\section{Lochung B}

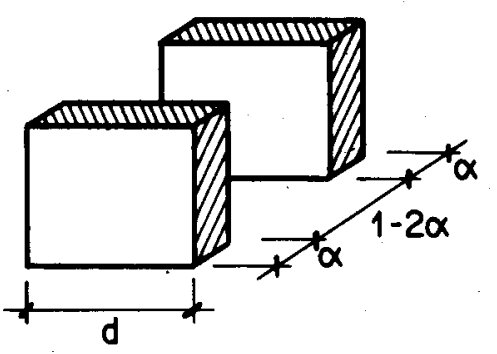

\section{Bild 4.16: 2extreme Lochungen}

- Lochung A: Die gesamte Lochfläche $d \cdot(1-2 \alpha)$ wird um die Mittelaxe des Steins konzentriert; der Stein besteht demnach aus zwei aussenliegenden Flanschen.

- Lochung B: Der Stein wird durch quer zur Mittelaxe laufende Lamellen gebildet, deren Gesamtfläche $2 \cdot \alpha \cdot d$ derjenigen von Lochung $A$ entspricht.

Der Lochflächenanteil Lo beträgt bei beiden Lochungen 1-2 $\alpha$.

Mit diesen zwei extremen Annahmen über die Lochung sind viele mögliche Fälle abgedeckt. Dis analytischen Beziehungen zwischen $8, P, \Phi$ und $M$ sind im $A n-$ hang A4 zusammengestellt. 


\section{5 .1 e- $\Phi$-Kurven}

In Bild 4.17 sind für zwei verschiedene Axiallasten $\left(P / P_{b}=0.10\right.$ und 0.50$)$ die e- $\Phi$-Kurven dargestellt. Die stark ausgezogenen Kurven entsprechen denjenigen für den Vollquerschnitt $(\alpha=0.5)$ und sind damit für beide Lochungen $A$ und $B$ identisch ( $v g l$. auch Bild 4.7). Sofern aber $\alpha<0.5$ ist, spielt die Lochung für den Verlauf der e- $\Phi-K u r v e$ eine grosse Rolle. Die der Lochung $A$ entsprechenden Kurven sind gestrichelt und diejenigen, die der Lochung $B$ entsprechen, strichpunktiert dargestellt.

Die e- $\Phi-K u r v e n$ der Lochung $A$ ergeben bei jeweils gleicher Krümmung stets grössere Exzentrizitäten, d.h. die Sekantenbiegesteifigkeit des Querschnittes ist bei Lochung A grösser als bei Lochung $B$ (für gleiche $\alpha$-Werte). Besonders deutlich ist dieser Sachverhalt bei $P / P_{b}=0.1$ und einem $\alpha$-Wert von 0.2 .

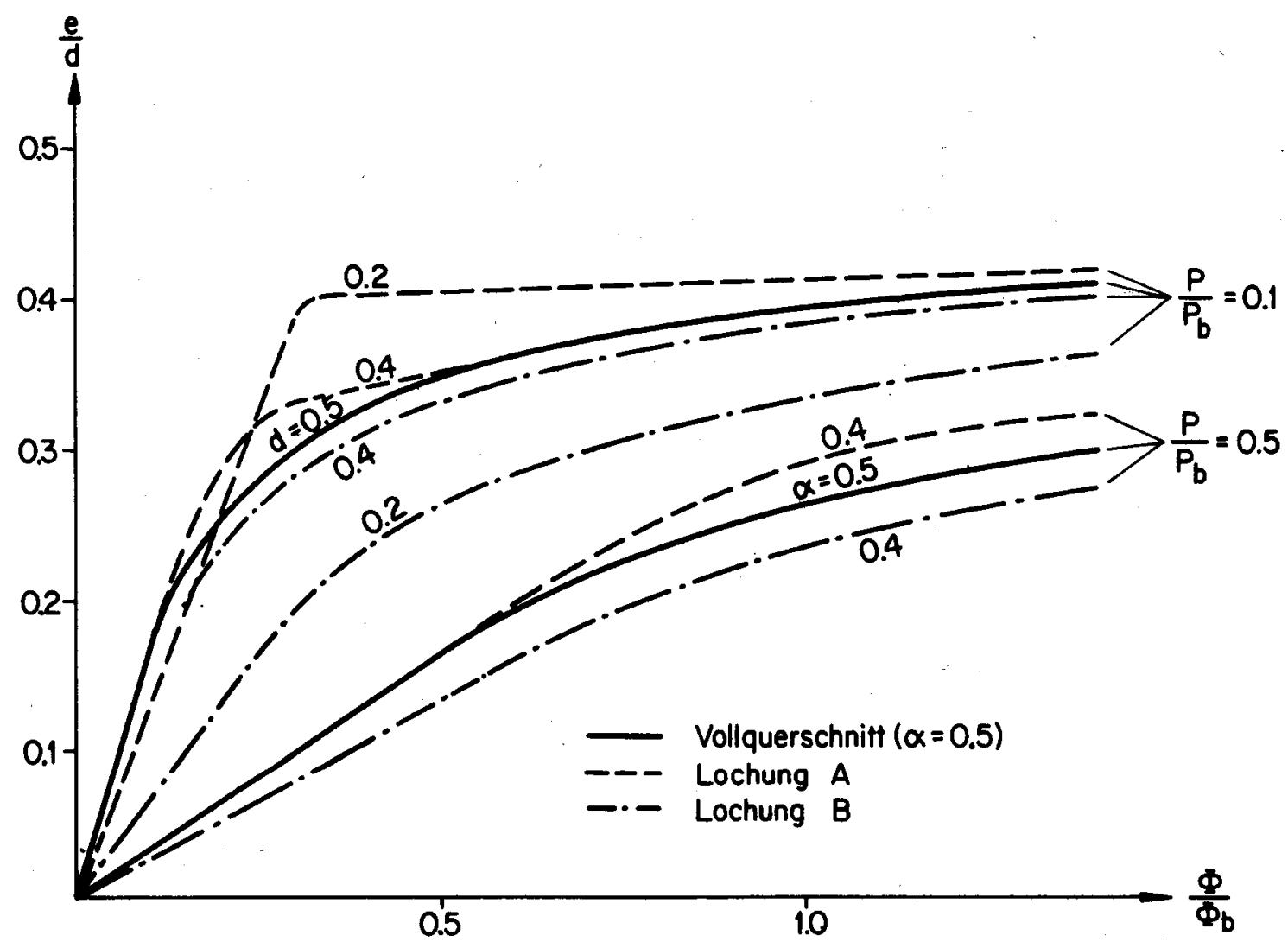

Bild 4.17: e $-\Phi-$ Kurven 


\section{5 .2 Zusammenhang $P-\Phi$}

In Bild 4.18 ist die Axiallast $P$ in Abhängigkeit der Krümmung $\Phi$ für den Vollquerschnitt sowie für Querschnitte mit der Lochung $A$ bzw. B $(\alpha=0.3)$ aufgetragen. Die dargestellten Kurven erfüllen dabei folgende Bedingungen:
a) $\sigma_{r}=\sigma_{b}$
b) $\varepsilon_{\mathrm{rz}}=0$
c) $\varepsilon_{r z}=-0.3 \cdot \varepsilon_{b}$

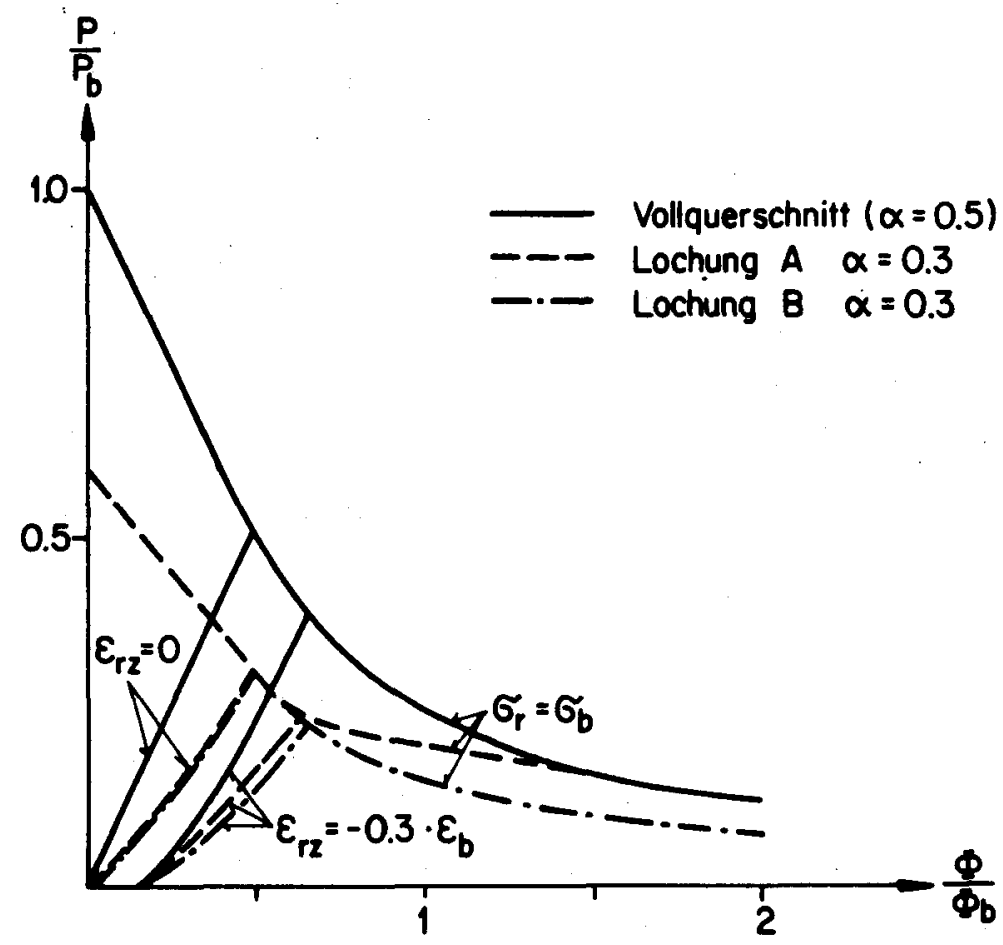

Bild 4.18: $P-\Phi$

Es zeigt sich, dass beim Vollquerschnitt (ausgezogene Kurven) die Krümmungen, bei denen $\sigma_{\Gamma}$ gleich $\sigma_{b}$ ist, grösser sind als bei Querschnitten mit Lochung $A$ (gestrichelt) und $B$ (strichpunktiert). Demgegenüber wird bei gegebener Axiallast die Randdehnung $\varepsilon_{r z}$ bei kleineren Krümmungen gleich null bzw. gleich $-0.3 \cdot \varepsilon_{b}$. 


\subsubsection{Interaktion M-P}

In Bild 4.19 sind für die Lochungen $A$ und $B(\alpha=0.2,0.4$ und 0.5$)$ die Interaktionskurven und die dazugehörigen Verläufe der Exzentrizität dargestellt. Den Kurven entlang ist $\sigma_{r}=\sigma_{b}$. Bei gegebener Axiallast nimmt das Moment mit abnehmendem $\alpha$ ab. Die Abnahme ist bei der Lochung $B$ grösser als bei Lochung $A$. Bei verschwindender Axiallast schmiegen sich die Interaktionskurven an diejenige des Vollquerschnittes $(\alpha=0.5)$.

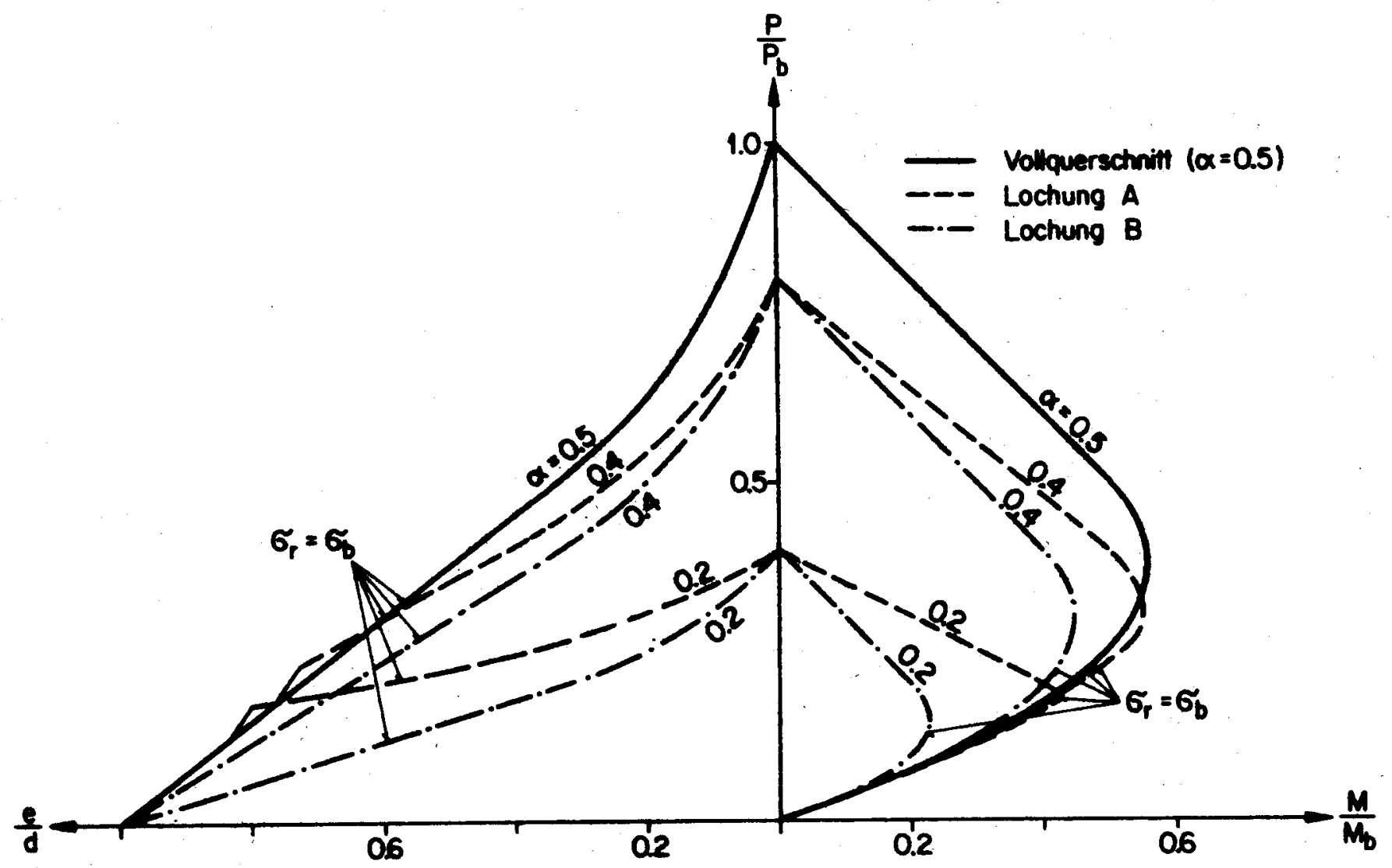

Bild 4.19: Interaktion $M-P$ 
- Beim linear elastischen Material ohne Zugfestigkeit kann die Exzentrizität die halbe Querschnittsdicke nicht überschreiten (Bild 4.7). Das Moment M und die Axiallast $P$ sind stets durch die Gl. (4.3) gekoppelt.

- Eine beschränkte Zugfestigkeit des Materials führt in den e- $\$$ - und den Interaktions-Kurven nur bei geringer Axiallast zu Abweichungen (vgl. Bilder 4.10 und 4.12 ).

- Der Einfluss der plastischen Verformbarkeit wird in den e- $\Phi$ - und den Interaktions-Kurven vor allem im Bereich grösserer Axiallasten sichtbar (vgl. Bilder 4.14 und 4.15 ).

- Die Lochung hat einen grossen Einfiuss auf die e-\$- und die InteraktionsKurven. Die Abweichungen gegenüber einem Vollquerschnitt lassen sich in zwei Anteile aufspalten: Einerseits nimmt der Lochflächenanteil Lo unabhängig von den Lochungen $A$ und $B$ mit abnehmendem $\alpha$ zu (vgl. Bild 4.16). Bei gleicher Axiallast ergeben sich damit im Querschnitt höhere Nettonormalspannungen. Dieser Einfluss kann aus dem Vergleich der Kurven für den Vollquerschnitt und derjenigen für den Querschnitt mit Lochung $B$ in den Bildern 4.17, 4.18 und 4.19 ermittelt werden. Andererseits führen die extremen Lochungen. A und $B$ für jeweils gleiche $\alpha$ ebenfalls zu abweichenden Kurvenverläufen. 
5. Vergleich von experimentellen und rechnerischen $e-\Phi-K u r v e n$ von Backsteinmauerwerk

In der Einleitung wurde auf das umfangreiche Versuchsprogramm im Rahmen des Forschungsprojektes "Rotationsfähigkeit von Mauerwerk hingewiesen. Es umfasste insgesamt 38 Versuche an Backsteinmauerwerk [7] und 16 Versuche an Kalksandsteinmauerwerk [8].

Auf der Grundlage der experimentell bestimmten e- $\Phi-K u r v e n$ wurde ein Stoffgesetz für das untersuchte Backsteinmauerwerk ermittelt. Damit konnten, unter Berücksichtigung der Steinlochung rechnerisch e- $\Phi$-Kurven bestimmt werden. Der Vergleich zwischen den rechnerischen und experimentellen Werten ergab eine gute Uebereinstimmung.

\subsection{Experimentelle e- $\Phi$-Kurven}

Die auf eine Betonplatte aufgemauerte Wand war an ihrem oberen und unteren Ende mittels Linienkipplager gelenkig gelagert. Unter konstanter Axiallast wurde das untere Wandende mittels Stahlrahmen schrittweise bis zum Bruch verdreht (Bild 5.1).

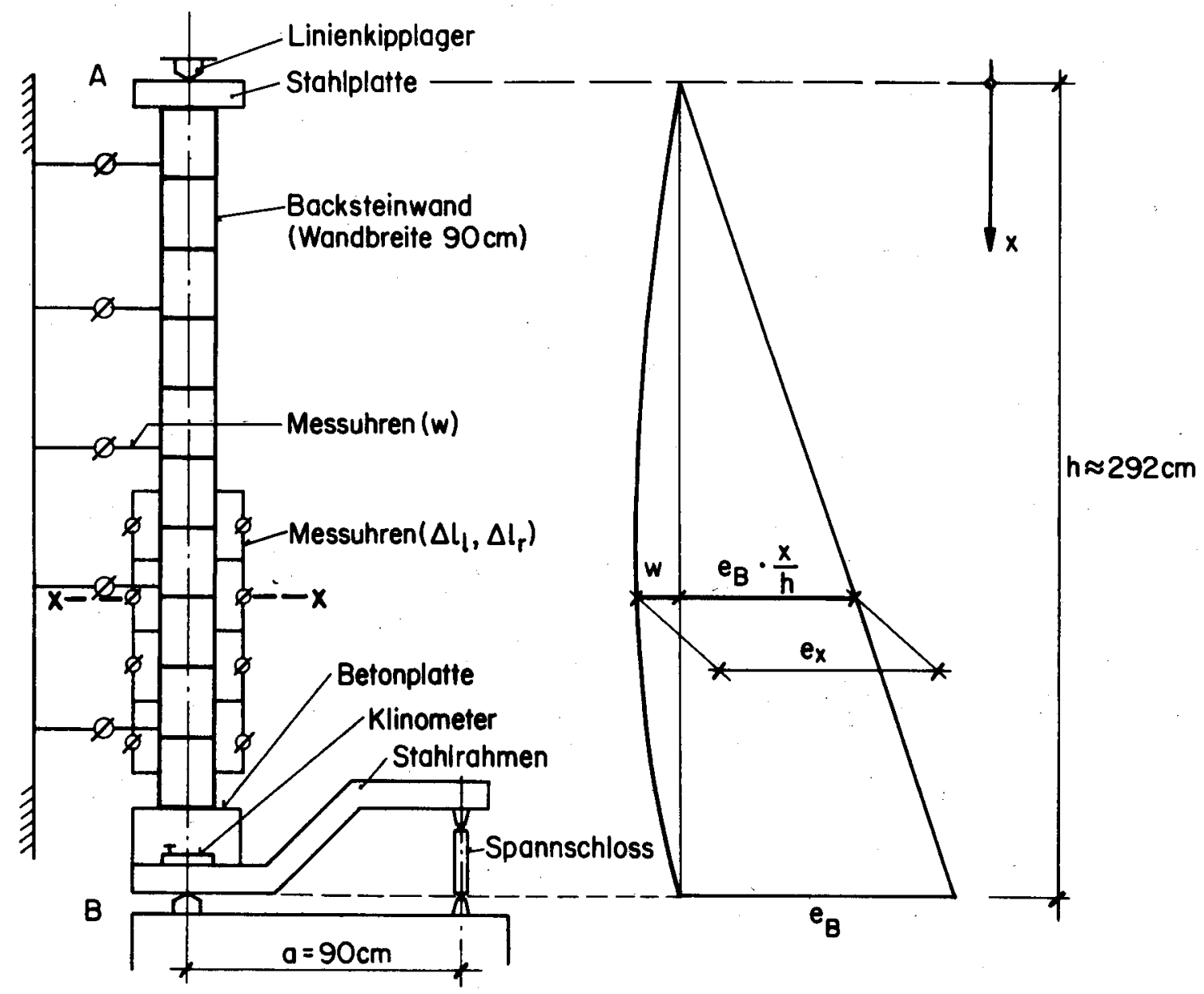

Bild 5.1: Versuchsanlage, Exzentrizität der Axiallast 


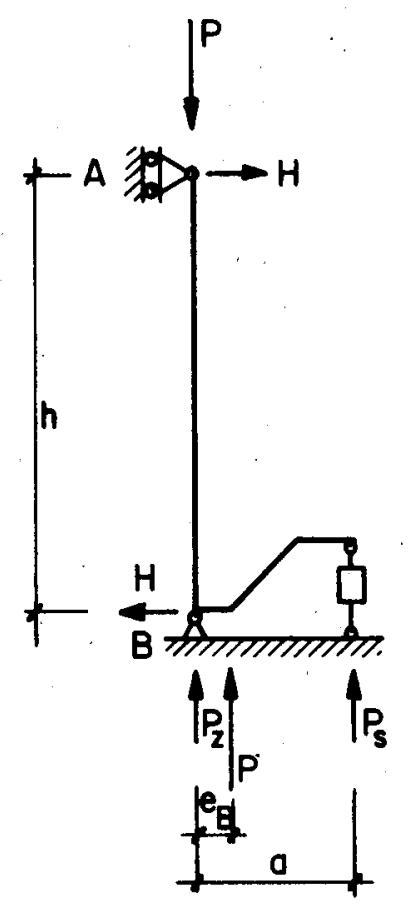

Bild 5.2 : Statische Verhältnisse

Die anfänglich zentrische Last $P\left(P=P_{z}\right)$ stellte sich bei einer Verdrehung des Stahlrahmens exzentrisch (Bild 5.2).

Im Spannschloss wurde die Kraft $P_{S}$ gemessen. Da $P$ konstant gehalten wurde, verminderte sich die zentrische Last $P_{z}=P$ um $P_{5}$. Bezüglich des unteren Linienkipplagers (Punkt $B$ ) ergab sich damit folgende Gleichgewichtsbedingung:

Punkt B: $\quad e_{B}=a \cdot \frac{P}{P}$

Bei der exzentrischen Lage von $P$ traten aus Gleichgewichtsgründen horizontale Reaktionen $H$ in den Linienkipplagern auf. Deren Grösse konnte wiederum aus einer Gleichgewichtsbetrachtung bezüglich B bestimmt werden:

Punkt B: $H=P_{S} \cdot \frac{a}{h}=P \cdot \frac{B}{h}$

Die Exzentrizität $e_{B}$ nimmt mit zunehmender Wandhöhe linear ab und verschwindet am oberen Wandende ( $v g l$. Bild 5.1).

Durch Addition dieser Exzentrizität und der horizontalen Auslenkung w erhält man in einem beliebigen Schnitt $x-x$ die totale Exzentrizität $e_{x}$. Die horizontale Auslenkung w wurde mit seitlich der Wand angebrachten Messuhren bestimmt. Die Exzentrizität $e_{x}$ beträgt:

$e_{x}=e_{B} \cdot \frac{x}{h}+w$

Auf beiden Seiten der Wand waren je sechs Messuhren angebracht (Bild 5.3). 


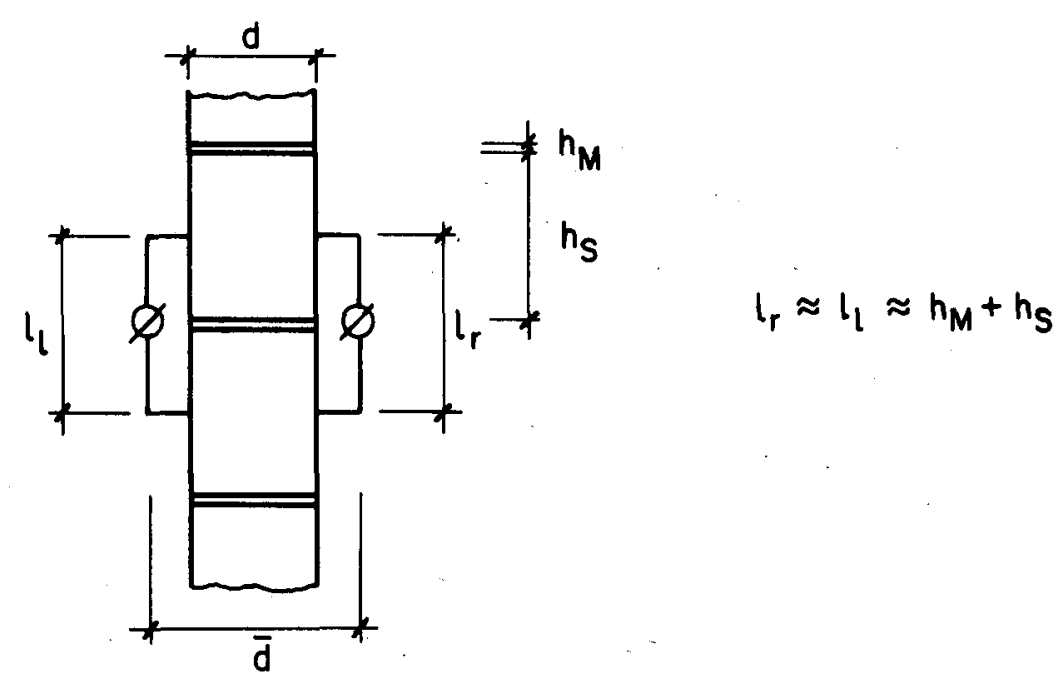

\section{Bild 5.3 : Deformationsmessung}

Die Messbasis erstreckte sich über eine Mörtelfuge und je zwei Steinhälften unter- und oberhalb der Mörtelfuge. Die Uhren dienten zur Messung der axialen Deformationen. Aus den Messwerten von je zwei gegenüberliegenden Uhren konnte die Krümmung bestimmt werden:

$\Phi=\frac{\frac{\Delta l_{1}}{l_{l}}-\frac{\Delta l_{r}}{l_{r}}}{\bar{d}}$

Damit konnte jedem Krümmungswert eine Exzentrizität zugeordnet werden. Die Krümmung $\Phi$ stellt einen Mittelwert der Krümmungen innerhalb des Messbereichs dar, und die Exzentrizität e wurde auf der Höhe der Mörtelfuge, d.h. in der Mitte des Messbereichs, bestimmt.

In Bild 5.4 sind solche Kurven von drei Versuchen für Axiallasten $P$ von 150 , 400 und $650 \mathrm{kN}$ dargestellt. Diese Versuchswände waren $15 \mathrm{~cm}$ dick und $90 \mathrm{~cm}$ breit. Die obigen Axiallasten entsprechen somit mittleren Bruttospannungen von $1.1,2.9$ und $4.8 \mathrm{~N} / \mathrm{mm}^{2}$. Die Wände bestanden aus hochwertigen Steinen $B H$ und Zementmörtel. Nach der Norm SIA 177, Art. 4.43, wird dieses Backsteinmauerwerk als MBHC bezeichnet [35] (vgl. Tabelle Mauerwerksgattungen im Anhang $A 1$ ).

In den horizontalen Mörtelfugen (Lagerfugen) ergaben sich bei der Verdrehung der Betonplatte Risse. Die Rissgrössen wurden gemessen und der im entsprechenden Messbereich bestimmten Exzentrizität und Krümmung zugeordnet. Die Zahlenwerte den e- $\Phi$-Kurven entlang geben die Rissweiten in Zehntelsmillimetern an. Haarrisse sind mit einem $H$ gekennzeichnet. In gleicher Weise wurden auch Abbräckelungen der Gipsschlämmschicht und Absplitterungen einzelner Steinplättchen erfasst und in den Kurven mit GS bzw. SS bezeichnet. Um die Rissentwicklung möglichst gut verfolgen zu können, wurden die Versuchswände vor dem Einbau jeweils mit einer weissen Gipsschlämmschicht bestrichen. Das Kurvenbild zeigt grundsätzlich den gleichen Verlauf wie die in Bild 4.7 dar- 


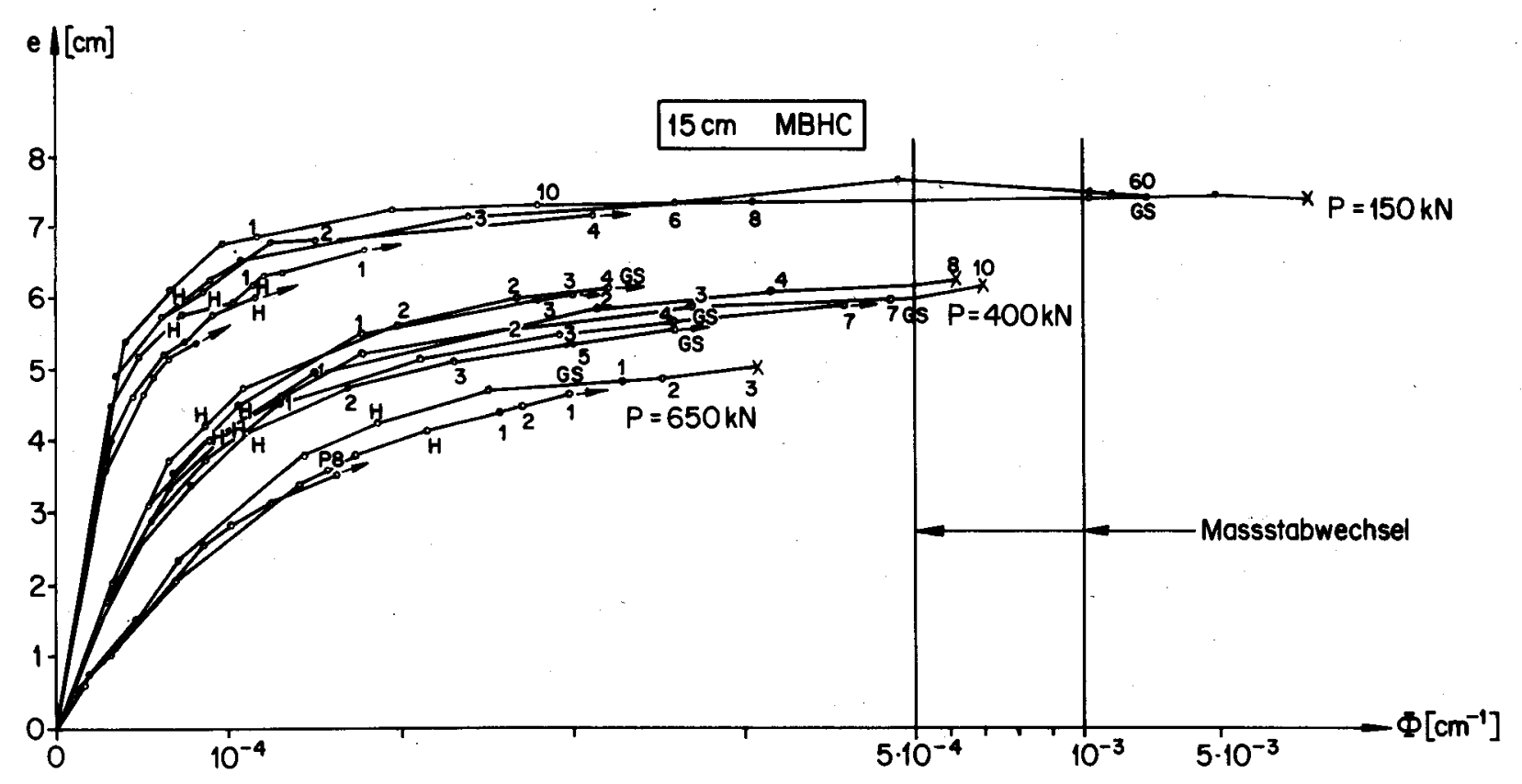

Bild 5.4 : Experimentelle e- $\Phi-$ Kurven

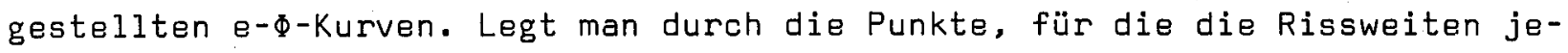
weils gleiche Werte aufweisen, näherungsweise eine Kurve, so erkennt man, dass diese ähnlich verläuft wie die Kurven in Bild 4.7, entlang denen die Randdehnung konstant ist. Die Bruchkrümmungen nehmen mit zunehmender Axiallast ab.

\subsection{Stoffgesetz für Backsteinmauerwerk}

In Kapitel 4 wurden auf der Grundlage verschiedener Annahmen für das Stoffgesetz und die Lochung die e- $\Phi$-Kurven diskutiert. Im vorliegenden Fall wurde der umgekehrte Weg beschritten: Aus dem Verlauf der experimentell bestimmten e- $\Phi-K u r v e n$ wurde durch Probieren ein nominelles Stoffgesetz für das untersuchte Mauerwerk abgeleitet. Es ist in Bild 5.5 dargestellt.

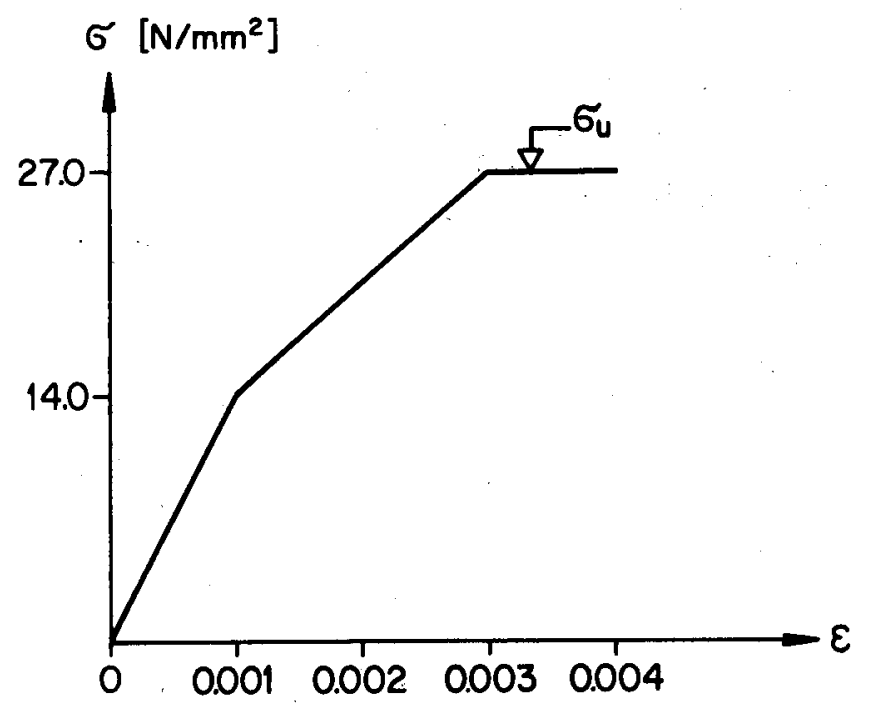

Bild 5.5: Stoffgesetz für Backsteinmauerwerk (MBHC) 
Es handelt sich um den Zusammenhang zwischen Nettospannungen und Stauchungen. Der linear ansteigende Kurvenast erfährt bei einer Nettospannung von $14 \mathrm{~N} / \mathrm{mm}^{2}$ bzw. einer Stauchung von 0.001 eine Abflachung. Bei $27 \mathrm{~N} / \mathrm{mm}^{2}$ schliesst ein horizontaler Ast an. Im Abschnitt 4.3 wurde gezeigt, dass sich bei vorhandner Zugfestigkeit $(\zeta=0.1,0.2)$ unter geringen Axiallasten in den e- $\Phi-K u r-$ ven ein deutlicher Knick ergab (Bild 4.10). Da ein solcher in den experimentellen Kurven nicht deutlich festgestellt werden konnte, wurde die Zugfestigkeit vernachlässigt. Der E-Modul bzw. die Neigung des anfänglich linear verlaufenden Kurvenastes konnte aus dem Anfangsverlauf der experimentellen e- $\Phi-$ Kurven bestimmt werden. Die Maximalspannung $\sigma_{U}=27 \mathrm{~N} / \mathrm{mm}^{2}$ ergab sich aus dem Vergleich von rechnerischen und experimentellen Kurven. Durch die Abflachung konnte die in den experimentellen Kurven festgestellte Ausrundung im Bereich grösserer Axiallast erreicht werden.

5.3 Gegenüberstellung von experimentellen und rechnerischen e- $\Phi-K u r v e n$

Auf der Grundlage des Stoffgesetzes von Bild 5.5 können nun rechnerisch für beliebige Lochungen und S.teinformate e- $\Phi$-Kurven bestimmt werden.

Für die numerische Berechnung wird der Querschnitt in Querrichtung in Lamellen aufgeteilt. Ausgehend von einer vorgegebenen Randstauchung $\varepsilon_{r} b z w$. Randdehnung $\varepsilon_{r z}$ und Axiallast $P$ wird die Neigung der Dehnungsebene bzw. die Krümmung durch ein Iterationsverfahren so bestimmt, dass der Absolutwert der Differenz von $P$ und resultierender Normalkraft (Summe der Lamellenkräfte) einen vorgegebenen Betrag unterschreitet. In einem zweiten Schritt wird die Summe der Momente der Lamellenkräfte bezüglich der Mittelaxe bestimmt. Durch Division dieser Momentensumme durch $P$ erhält man die Exzentrizität e. Damit ist ein Punkt der e- $\Phi$-Kurve bei vorgegebener Randstauchung $\varepsilon_{r}$ und Axiallast bestimmt. Dieses Berechnungsverfahren kann nun für weitere Werte von $\varepsilon_{\Gamma}$ wiederholt werden.

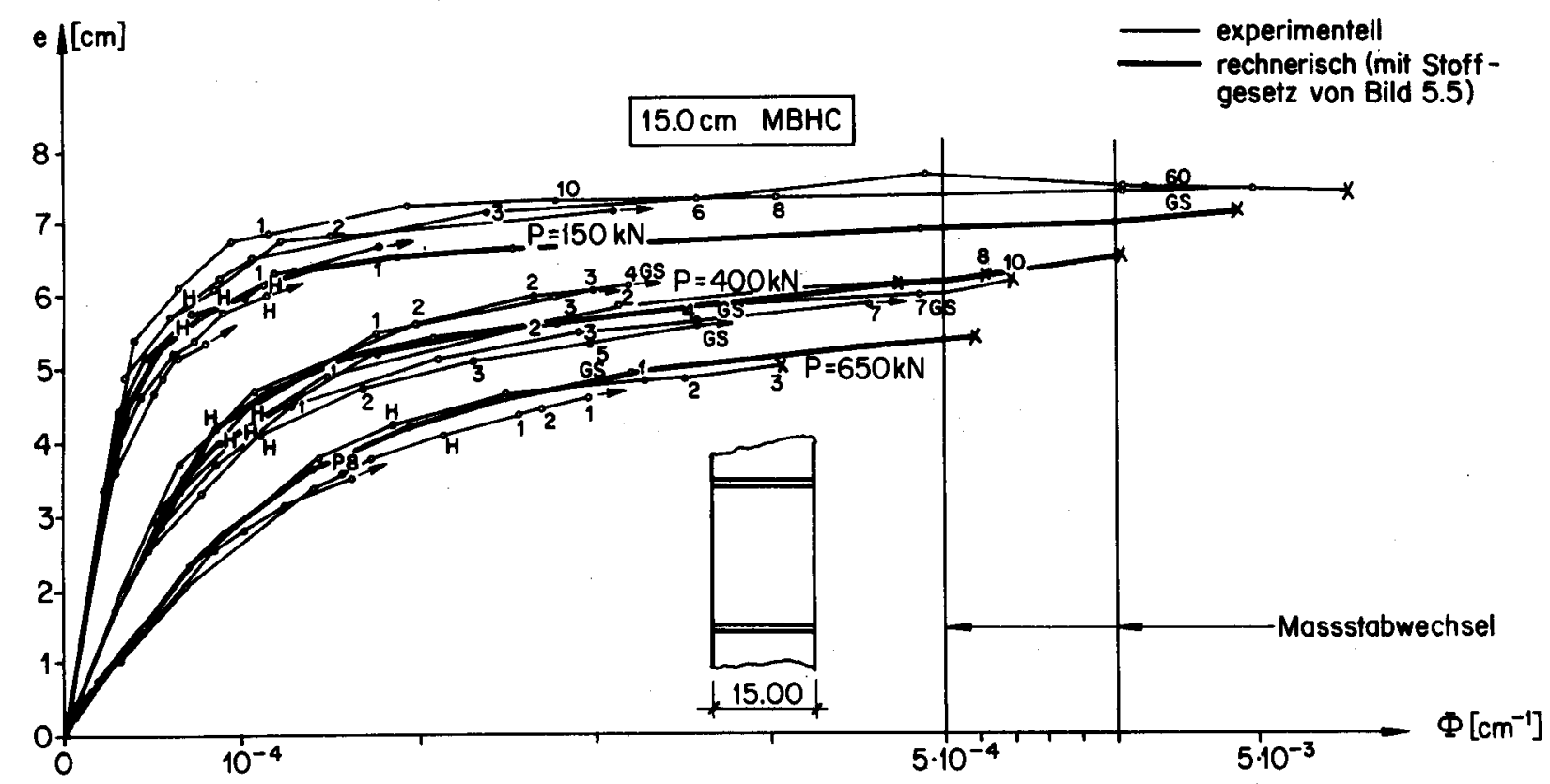

Bild 5.6: Experimentelle und rechnerische e- $\Phi$-Kurven 

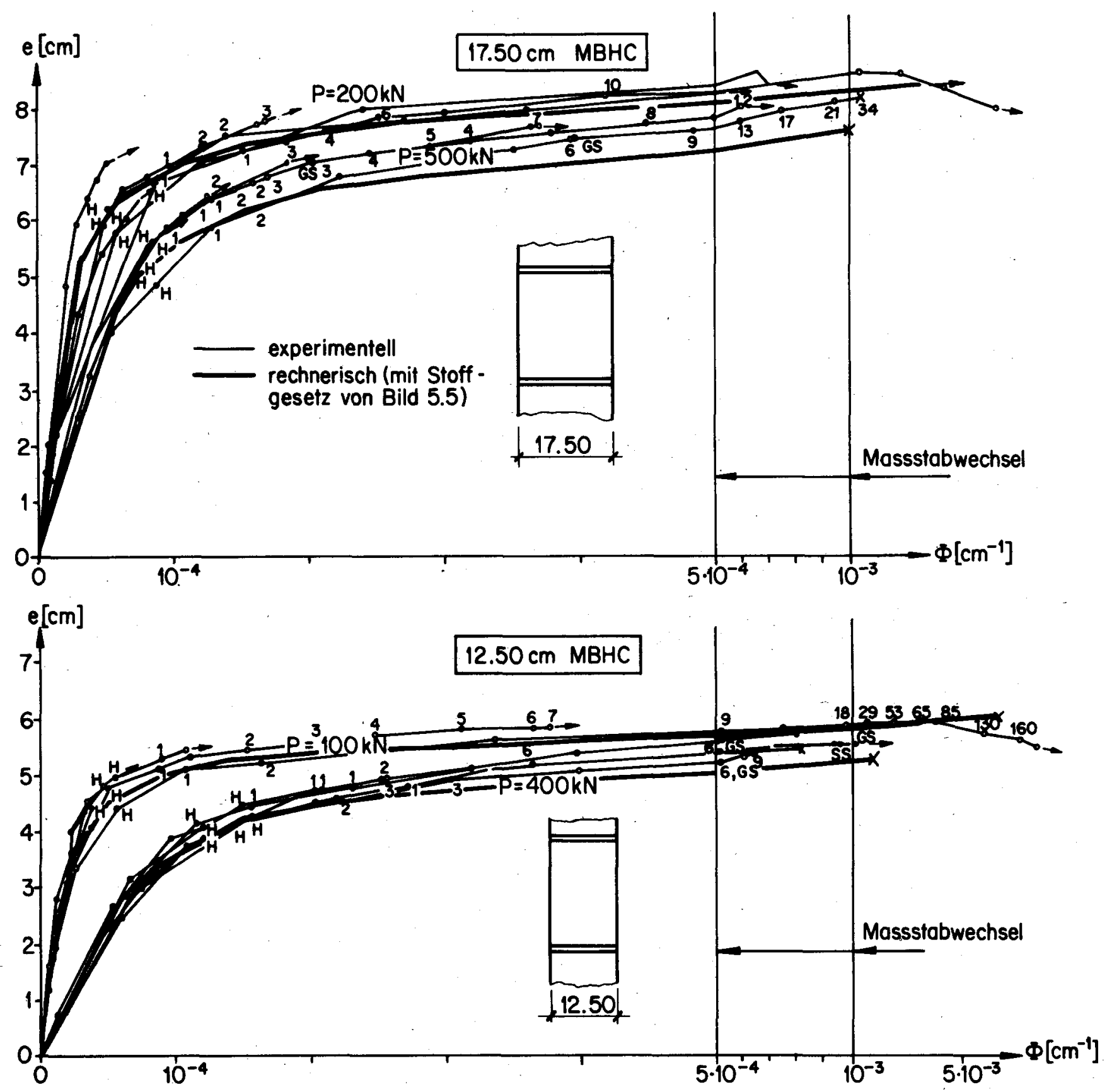

Bild 5.7 : Experimentelle und rechnerische e- $\Phi$-Kurven

Die Bilder 5.6 und 5.7 zeigen eine Gegenüberstellung von rechnerischen und experimentellen Kurven. In Bild 5.6 ist eine solche für die Kurven von Bild 5.4 dargestellt. Es handelt sich dabei um das $15 \mathrm{~cm}$ Backsteinmauerwerk MBHC. In Bild 5.7 sind rechnerische und experimentelle Kurven für das 17.50 und $12.50 \mathrm{~cm}$ Backsteinmauerwerk dargestellt. Die Axiallasten betragen dabei 200 und 500 bzw. 100 und $400 \mathrm{kN}$ und die mittleren Bruttospannungen $\sigma_{\mathrm{m}} 1.27$ und 3.17 sowie 0.88 und $3.55 \mathrm{~N} / \mathrm{mm}^{2}$. Die Steinhöhe betrug in allen drei Fällen $19 \mathrm{~cm}$. Bild 5.8 zeigt die Lochungen der drei BH-Steine.

Die Uebereinstimmung der rechnerischen und experimentellen werte ist gut. 
$\mathrm{BH}-12.50 \mathrm{~cm}$

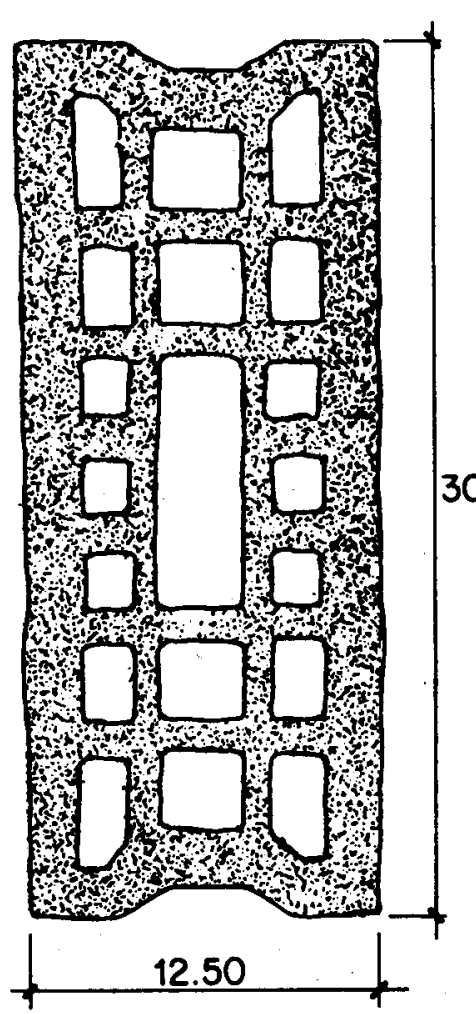

$\mathrm{BH}-15.00 \mathrm{~cm}$

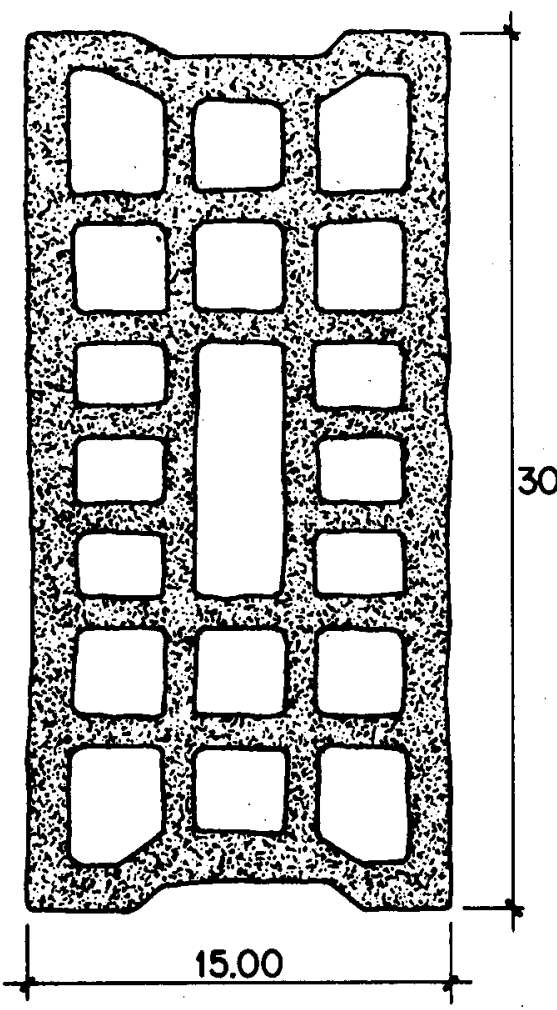

$\mathrm{BH}-17.50 \mathrm{~cm}$

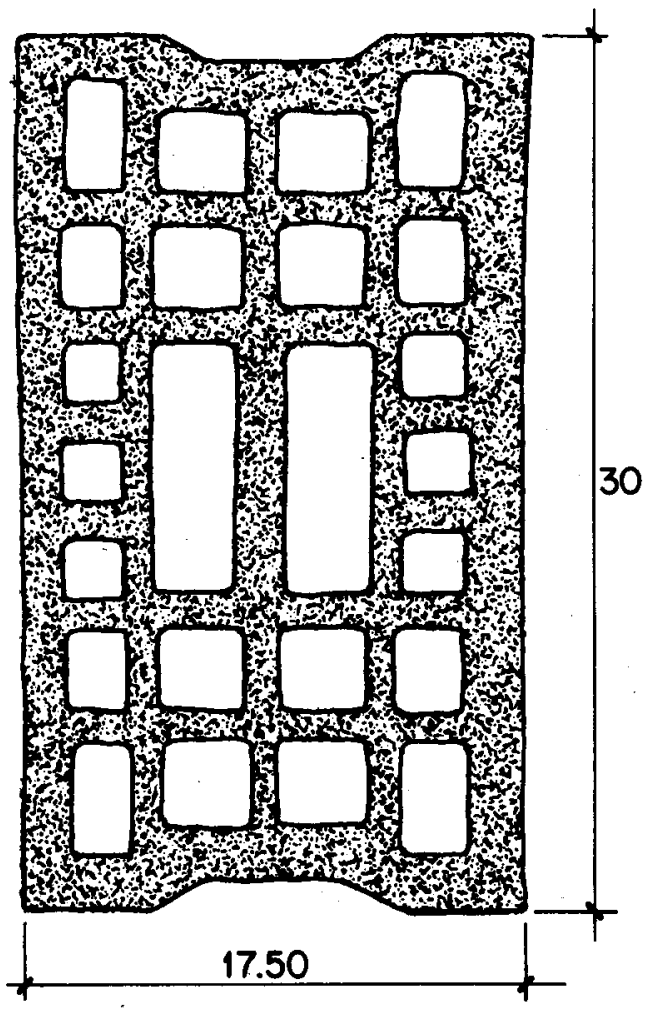

Bild 5.8: Lochungen der $\mathrm{BH}$ - Steine

Im Folgenden sollen am Beispiel des $15 \mathrm{~cm}$ Backsteinmauerwerks MBHC einige weitere Vergleiche zwischen rechnerischen und experimentellen Werten gezeigt werden. Es handelt sich dabei um die Ermittlung einer rechnerischen Bruchstauchung sowie um genauere Abklärungen über den Zusammenhang von experimentell beobachteten Risskrümmungen und rechnerischen Randdehnungen.

In Bild 5.9 sind die experimentellen Bruchkrümmungen bei der jeweils vorhandenen Axiallast punktweise eingetragen. Daneben sind rechnerische Kurven gleicher Randstauchung $\varepsilon_{r}$ für $0.001,0.002,0.003$ und 0.004 dargestellt. Bei kleinen Axiallasten $(P \leq 250 \mathrm{kN})$ übersteigen die bei der Bruchkrümmung vorhandenen rechnerischen Randstauchungen die Werte von 0.004 deutlich. Bei mittleren Axiallasten ( $(P=400 \mathrm{kN})$ beträgt die Bruchrandstauchung ca. 0.003 und bei grossen Axiallasten ( $P \geq 650 \mathrm{kN}$ ) entsprechen den eingetragenen Bruchkrümmungen Randstauchungen von ca. 0.002. Zusätzlich sind die Krümmungswerte, bei denen einzelne Steinplättchen abplatzten bzw. einzelne Teile der Gipsschlämmschicht abbröckelten, eingetragen. Es zeigte sich, dass bei Randstauchungen von ca. 0.003 mit Steinabsplitterungen und bei 0.001 bis 0.002 mit Abbröckelungen der Gipsschlämmschicht zu rechnen ist. 


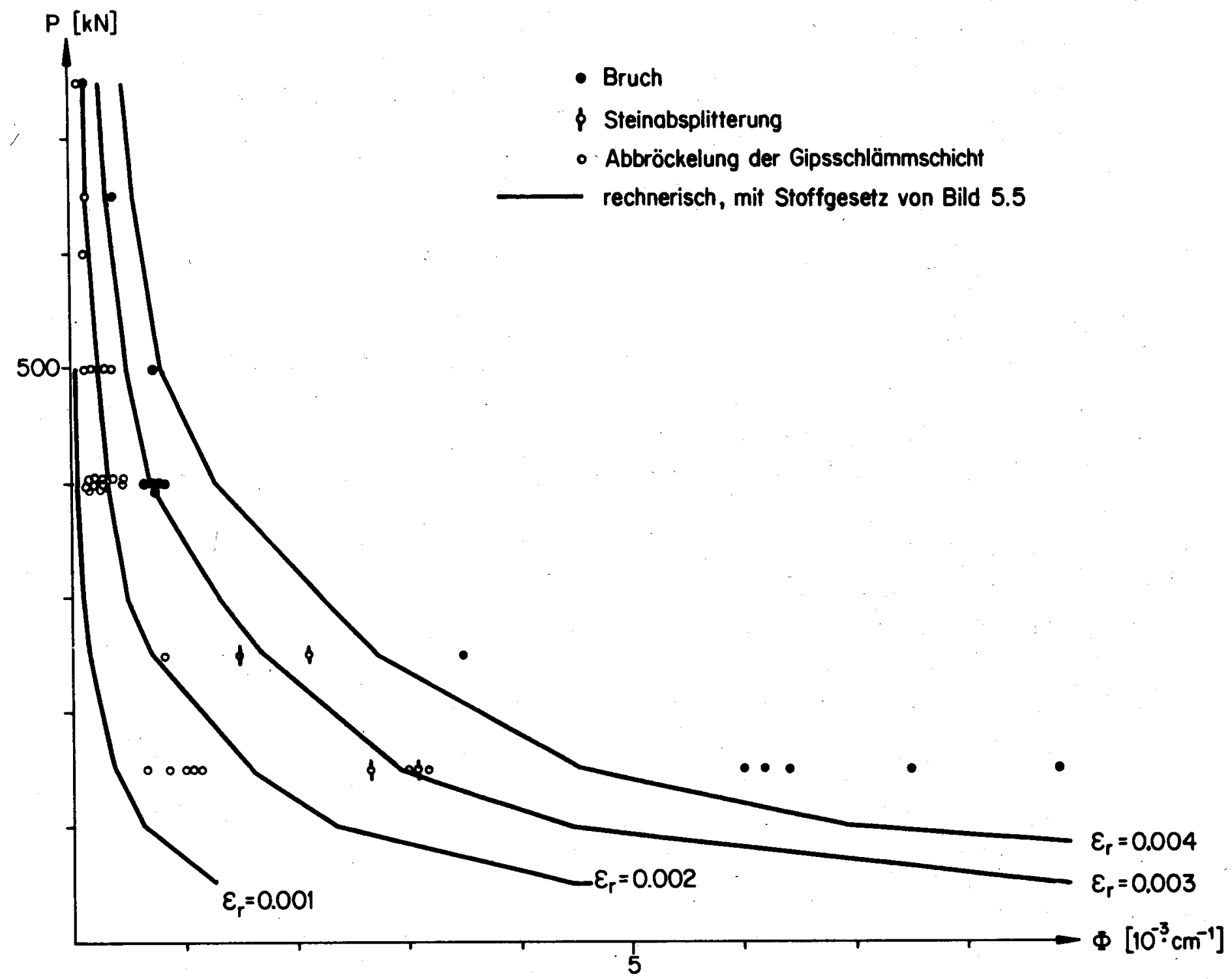

Bild 5.9: $P-\Phi$ : experimentell- rechnerisch $(15 \mathrm{~cm} \mathrm{MBHC})$

In Bild 5.10 sind die experimentell bestimmten Krümmungen, bei denen sich Risse von $0.05 \mathrm{~mm}$ (Haarrisse), Risse von 0.3 und von $0.5 \mathrm{~mm}$ in der Lagerfuge ergaben, bei der jeweils vorhandenen Axiallast eingetragen. Die Risse bildeten sich ausschliesslich in den Lagerfugen. Innerhalb des Messbereichs ergab sich in der Regeliein Riss, in einzelnen Fällen bildete sich beidseits der Lagerfuge zwischen Stein und Mörtel je ein Riss.

Das diesen Untersuchungen zugrunde gelegte Stoffgesetz besitzt keine Zugfestigkeit. Im Querschnitt sich ergebende Randdehnungen entsprechen demzufolge spezifischen Rissweiten. Werden die Randdehnungen über den ganzen Messbereich (200 mm) integriert, so erhält man eine rechnerische Rissweite $r$ :

$r=200 \cdot \varepsilon_{\mathrm{rz}} \quad(\mathrm{r}$ in $\mathrm{mm})$

Rissweiten von $0.05,0.3$ und $0.5 \mathrm{~mm}$ entsprechen somit Randdehnungen von $0.00025,0.0015$ und 0.0025 . In Bild 5.10 sind die rechnerischen Kurven, denen 


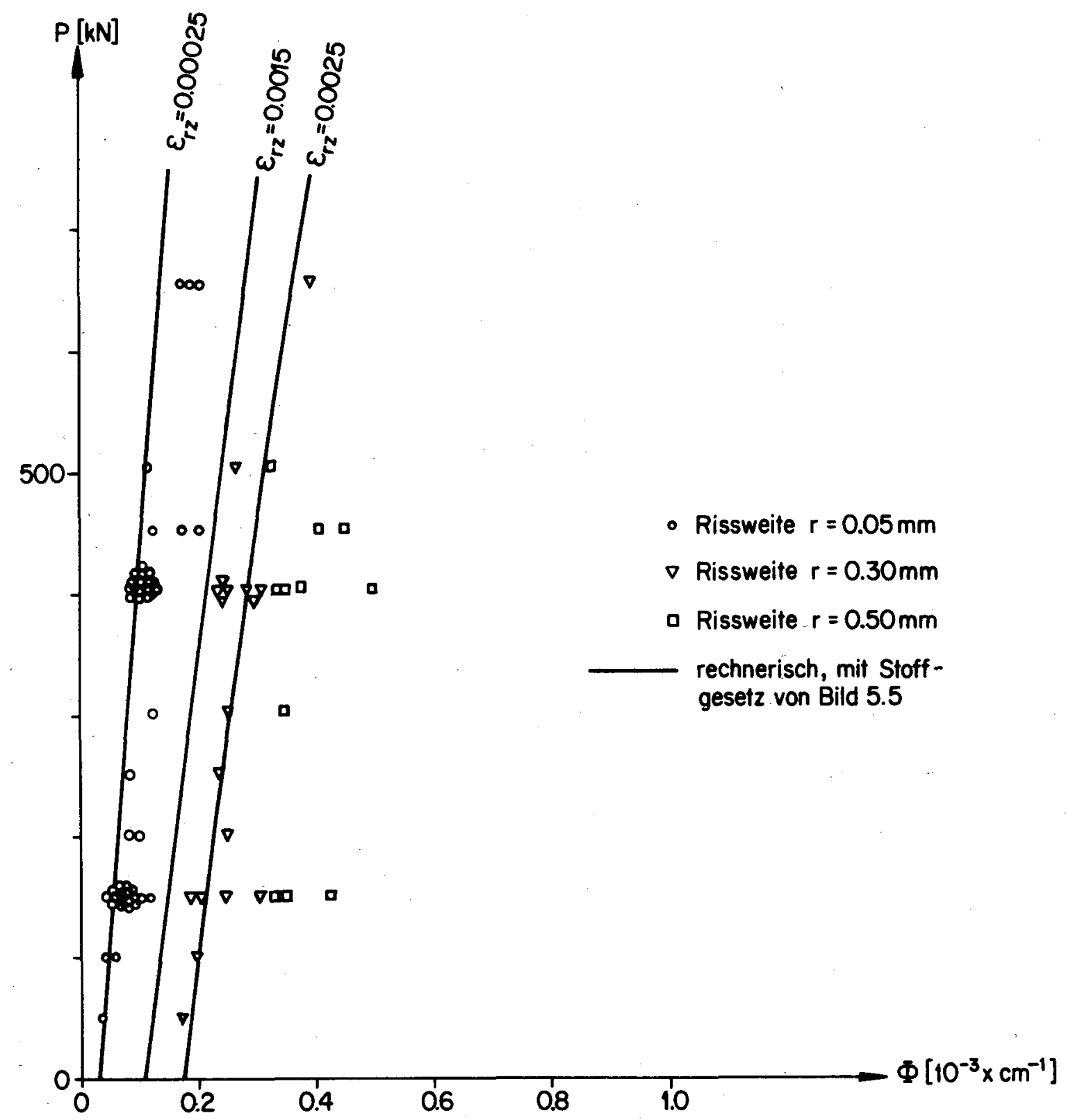

Bild 5.10: $P-\Phi$ : experimentell - rechnerisch (15 cm MBHC)

entlang die Randdehnung $\varepsilon_{r z}$ diese Werte aufweist, eingezeichnet. Die eingetragenen Versuchspunkte scharen sich um die entsprechenden Kurven. Die Kurven liegen tendenziell auf der linken Seite der Punktwolken. Dies ist einerseits darauf zurückzuführen, dass sich in einzelnen Fällen beiseits der Lagerfugen Risse bildeten, deren Rissweiten entsprechend kleiner waren und andererseits das Material eine kleine Zugfestigkeit hatte. In Abschnitt 4.3 wurde gezeigt, dass bei einem Material mit Zugfestigkeit gleich grosse spezifische Risse bzw. Randdehnungen erst bei grösseren Krümmungen auftreten als bei einem Material ohne Zugfestigkeit (Bild 4.11).

In Bild 5.11 sind die maximalen, beim Bruch der Wand gemessenen Exzentrizitäten punktweise dargestellt. Die Exzentrizitäten sind auf die Querschnittsbreite bezogen. Unter der Annahme einer rechnerischen Bruchstauchung von 0.002 und 0.004 ( vgl. Bild 5.9) ergeben sich in Abhängigkeit der Axiallast $P$ die eingezeichneten Exzentrizitätsverläufe. Der Einfluss der Steinlochung 


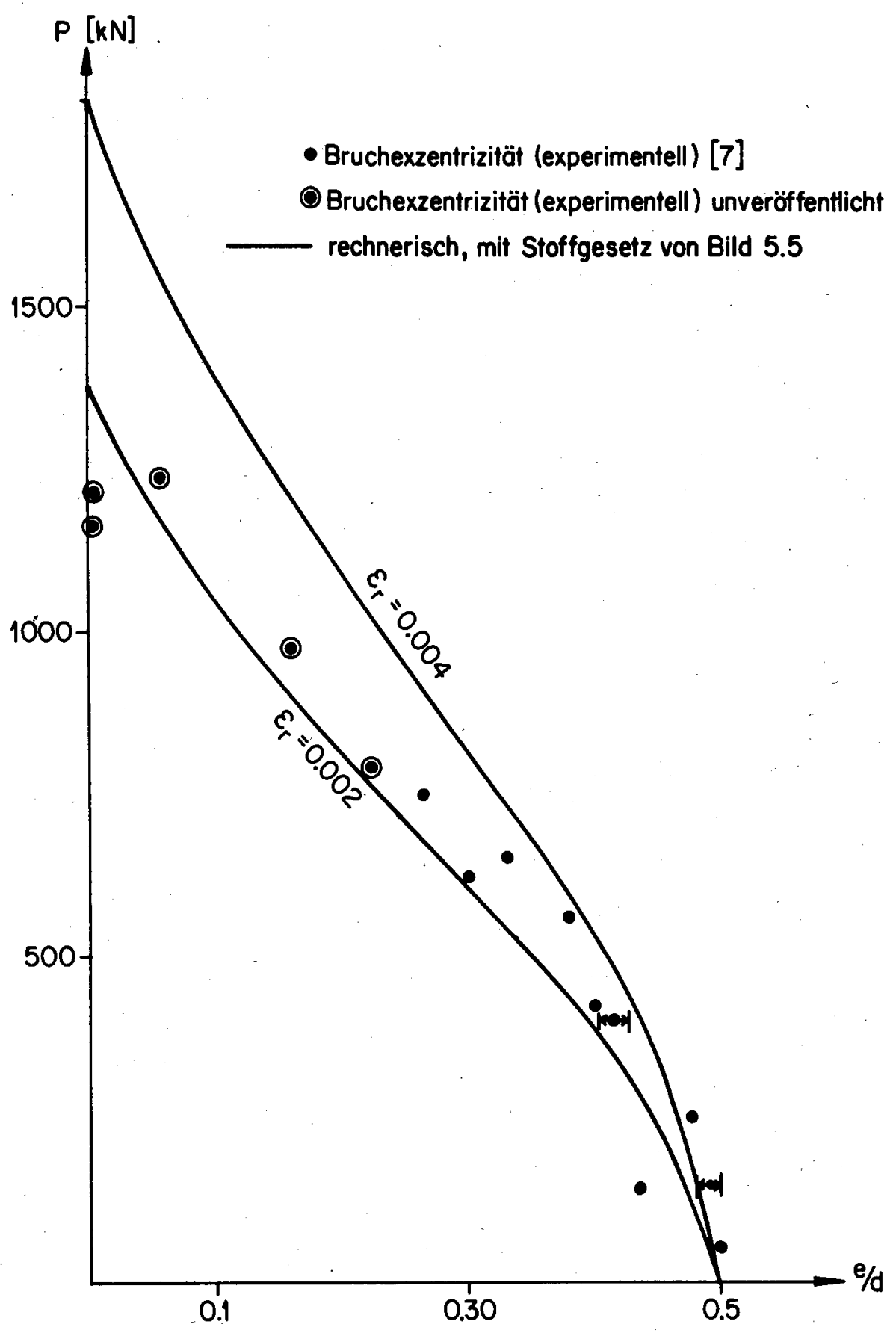

Bild 5.11: $\mathrm{P}-\mathrm{e}:$ experimentell- rechnerisch $(15 \mathrm{~cm} \mathrm{MBHC})$

kann aus dieser Darstellung deutlich erkannt werden. Durch die Konzentration des Materials an die Steinaussenseiten wird die Kurve, die den Zusammenhang zwischen der Exzentrizität und der Axiallast bei jeweils konstanter Randstauchung beschreibt, nach aussen hin verschoben (Bild 4.19, Abschnitt 4.5). Die experimentellen Werte werden durch die rechnerischen Kurven eingegabelt. Bei kleinen Axiallasten liegen die Punkte näher bei der 0.004-Kurve, und bei grossen entlang der die Bruchrandstauchung 0.002 beträgt. 


\section{Tragverhal ten von axial belasteten Mauerwerkswänden}

6.1 Ueberblick

Im Folgenden soll das Tragverhalten der in Bild 6.1 dargestellten Maverwerkswände untersucht werden. Es wird angenommen, dass die Wände axial und linienförmig belastet werden.

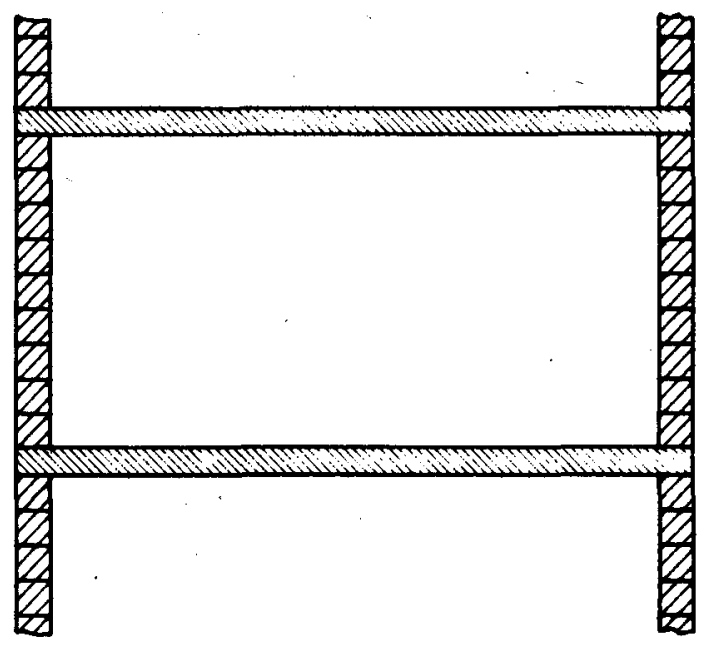

\section{Bild 6.1: Mauerwerkwände und Decken im Verbund}

Aus der konstruktiven Gestaltung ergeben sich dabei grundsätzlich zwei verschiedene Beanspruchungsarten der Wände:

\section{Beanspruchungsart E (Exzentrizität)}

Zwischen Dachdecke und Wand werden vielfach Gleitlager eingebaut (Bild 6.2). Die Last wird dadurch konzentriert über einzelne Punktlager oder Lagerstreifen eingeleitet. Je nach Wandkonstruktion (Einstein-oder Verbandmauerwerk) werden diese Gleitlager zentrisch oder exzentrisch verlegt. Die Exzentrizität e der Axiallast ist damit durch die konstruktive Durchbildung der Lger gegeben und von der Deckenverformung praktisch unabhängig.

Dachdecken neigen üblicherweise zu relativ grossen Verformungen infolge von Temperatureinflüssen und erhöhten differentiellen Schwindverkürzungen. Durch den Einbau von Gleitlagern kann die Zwängungsbeanspruchung in der Wand weitgehend vermieden werden. 


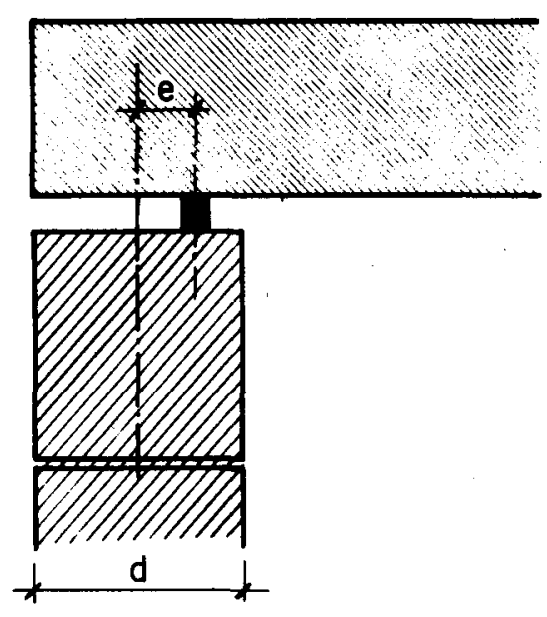

\section{Bild 6.2 : Lagerstreifen zwischen Decke und Wand}

\section{Beanspruchungsart $\bigvee$ (Verdrehung)}

In den meisten Fällen werden Betondecken auf die Wände betoniert (Bild 6.3). Die Last in der oberen Wand sowie die Deckenlast werden flächenförmig in die darunterliegende Wand übertragen. Die resultierenden Verformungen von Decke und Wänden ergeben sich aus den Steifigkeitsverhältnissen. Im Gegensatz zur Beanspruchungsart $E$ ist die Exzentrizität e verformungsabhängig.

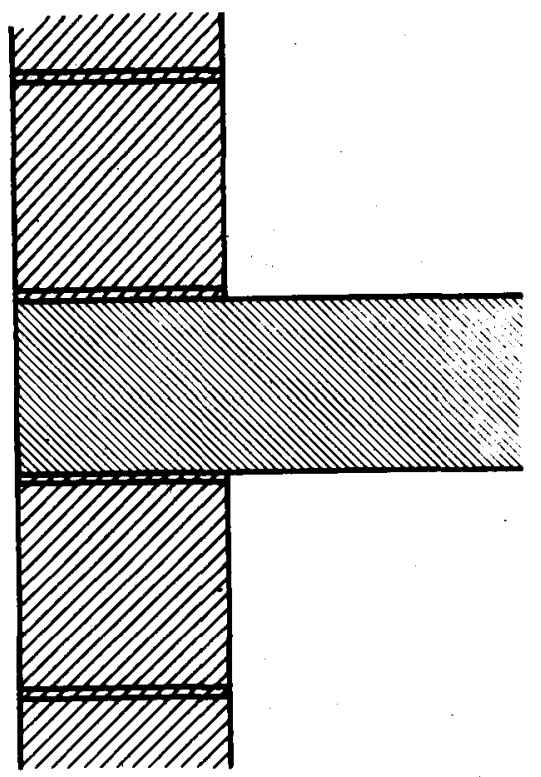

\section{Bild 6.3 : Direkte Lagerung der Decke}

Es sei nun die Mauerwerkswand in Bild 6.4 betrachtet. Sie ist durch die Linienlast $P$ axial belastet und ihre Schlankheit beträgt $\delta$. Die Schlankheit wird bei Mauerwerkswänden üblicherweise durch den Quotienten von Wandhöhe h und Wanddicke d definiert. Die Last $P$ greift bezüglich der Wandaxe exzentrisch an. Am oberen Wandende A betrage die Exzentrizität ${ }_{A}$ und am unteren $e_{B}$. Die bei dieser Beanspruchung sich ergebenden Verdrehungen der Wandenden sind $\vartheta_{A}$ und $\vartheta_{B}$. 


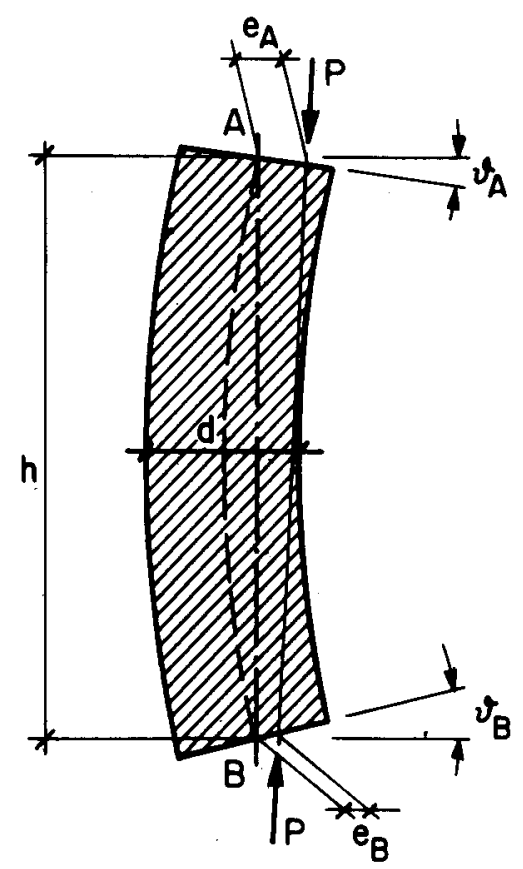

$\delta=\frac{h}{d}(6.1)$

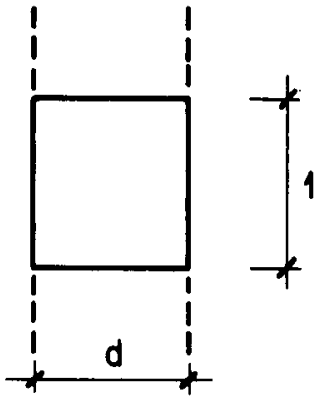

Bild 6.4 : Exzentrisch belastete Mauerwerkwand

Die Grössen $P, e_{A}, e_{B}$ bzw. $P, \vartheta_{A}, \vartheta_{B}$ bestimmen also die Beanspruchung der Wand in Bild 6.4 .

Bei der in den folgenden theoretischen Untersuchungen (Abschnitt 6.2) betrachteten wand handelt es sich um einen Wandabschnitt der Breite 1 (Bild $6.4)$.

Die Wand in Bild 6.5 sei wiederum durch die Linienlast $P$ axial belastet. Infolge der exzentrischen Lage von P bezüglich der Wandaxe ergeben sich hori-

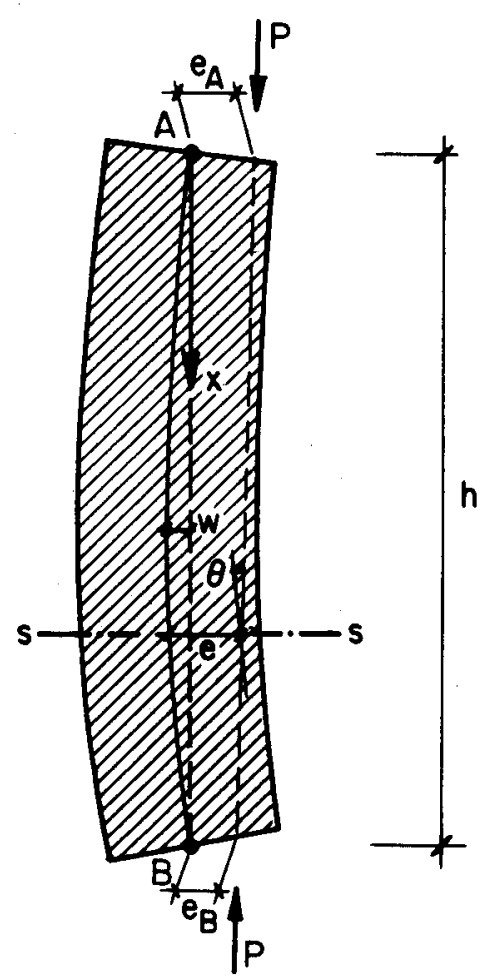

Bild 6.5 : Bezeichnungen 
zontale Auslenkungen w. Die totale Exzentrizität e in einem beliebigen

Schnitt s-s der Wand beträgt somit

$e=e_{A}+\frac{e_{B}-e_{A}}{h} \cdot x+w$

Befindet sich die Wand im Gleichgewicht, so ergeben sich die folgenden Bedingungen:

$$
\begin{aligned}
& M_{i}=\int_{Q S} \sigma \cdot y \cdot d f=M_{a}=P \cdot \theta \cdot \cos \theta \\
& N=\int_{Q S} \sigma \cdot d f=P \cdot \cos \theta \\
& Q=\int_{Q S} \tau \cdot d f=P \cdot \sin \theta
\end{aligned}
$$

$M_{i}$ ist durch die Krümmung im Schnitt s-s und die Axiallast $P$ bestimmt. $M_{a}$ ergibt sich durch Multiplikation von $P$ mit $e \cdot \cos \theta$. Unter der Annahme, dass die Neigung $\theta$ der Wirkungslinie von $P$ gegenüber der Wandaxe gering ist, d.h. $\cos \theta \approx 1$ und $\sin \theta \approx 0$, wird $N \approx P$ und $Q$ verschwindet.

Für ein linear elastisches Material führt die Gleichgewichtsbedingung ( 6.3 a) zu einer Differentialgleichung, die sich geschlossen lösen lässt. Angervo [2] hat 1954 die Lösung auf ein Material mit linear elastischem Stoffgesetz ohne Zugfestigkeit erweitert. Im Tragverhalten ergeben sich dabei erhebliche Abweichungen. Sie werden im Folgenden näher betrachtet.

\subsection{Analytische Lösungen}

\subsubsection{Linear elastisches Material}

Unter der Voraussetzung eines linear elastischen Stoffgesetzes ergibt sich zwischen dem Moment und der Krümmung folgender Zusammenhang:

$M_{i}=-E I \cdot w "$

Nach Einsetzen von $M_{i}$ in GI. (6.3 a) und zweimaliger Differentiation erhält man

$$
w^{\prime \prime}+w^{2} \cdot w "=0
$$

wobei die Grösse w eingeführt wurde

$\omega=\sqrt{\frac{P}{E I}}$ 
Der Lösungsansatz

$w=c_{1} \cdot \sin w x+c_{2} \cdot \cos w x+c_{3} \cdot w+c_{4}$

erfüllt die Differentialgleichung (6.5). Für die Bestimmung der vier Unbekannten $C_{1}, \ldots C_{4}$ stehen vier Randbedingungen (Bild 6.5) zur Verfügung:

$w(0)=0$

$$
\mathrm{C}_{2}
$$

$+c_{4}=0$

$w^{\prime \prime}(0)=-\frac{e_{A} \cdot P}{E I}$

$-c_{2} \cdot \omega^{2}$

$=-\frac{\lambda \cdot e_{A} \cdot P}{E I}$

$w(h)=0$

: $\quad C_{1} \cdot \sin w h$

$+c_{2} \cdot \cos \omega h+C_{3} \cdot h+c_{4}=0$

$w^{\prime \prime}(h)=-\frac{\lambda \cdot e_{A} \cdot P}{E I}:-C_{1} \cdot \omega^{2} \cdot \sin \omega h-C_{2} \cdot \omega^{2} \cdot \cos w h$

$=-\frac{\lambda \cdot e_{A} \cdot P}{E \bar{I}}$

Die Grösse $\lambda$ gibt das Verhältnis zwischen der grösseren und der kleineren Wandexzentrizität an. Der Definitionsbereich von $\lambda$ ist somit gegeben: $-1 \leq \lambda \leq 1$. Nach Auflösung des Gleichungssystems (6.8) erhëlt man folgende Beziehung:

$w=e_{A} \cdot\left\{\frac{\lambda-\cos \omega h}{\sin \omega h} \cdot \sin \omega x+\cos \omega x+\frac{x}{h} \cdot(1-\lambda)-1\right\}$

Die erste Ableitung von w nach $x$ entspricht an der Stelle $x=0$ und $x=h$ den Wandverdrehungswinkeln $\vartheta_{A}$ und $\vartheta_{B}$ :

$\vartheta_{A}=e_{A} \cdot\left\{\frac{\lambda-\cos \omega h}{\sin \omega h} \cdot \omega+\frac{1-\lambda}{h}\right\}$

Die Exzentrizität in einem beliebigen Schnitt $x$ der Wand wird nach Einsetzen von $w$ und $\lambda$ in GI. $(6.2)$

$e=e_{A} \cdot\left\{\frac{\lambda-\cos \omega h}{\sin \omega h} \cdot \sin \omega x+\cos \omega x\right\}$

Der ort $\bar{x}$ der maximalen Exzentrizität e ergibt sich aus den Nullstellen der ersten Ableitung.

$$
\begin{aligned}
\frac{d e}{d x}= & e_{A} \cdot\left\{\frac{\lambda-\cos \omega h}{\sin \omega h} \cdot \omega \cdot \cos \omega x-\omega \cdot \sin \omega x\right\}=0 \\
& \tan \omega \bar{x}=\frac{\lambda-\cos \omega h}{\sin \omega h}
\end{aligned}
$$

Die maximale Exzentrizität $e_{\max }$ beträgt damit

$$
e_{\max }=\frac{e_{A}}{\cos \omega \bar{x}}
$$




\section{$6: 2 \cdot 1 \cdot 1$ Symmetrische Wandbelastung}

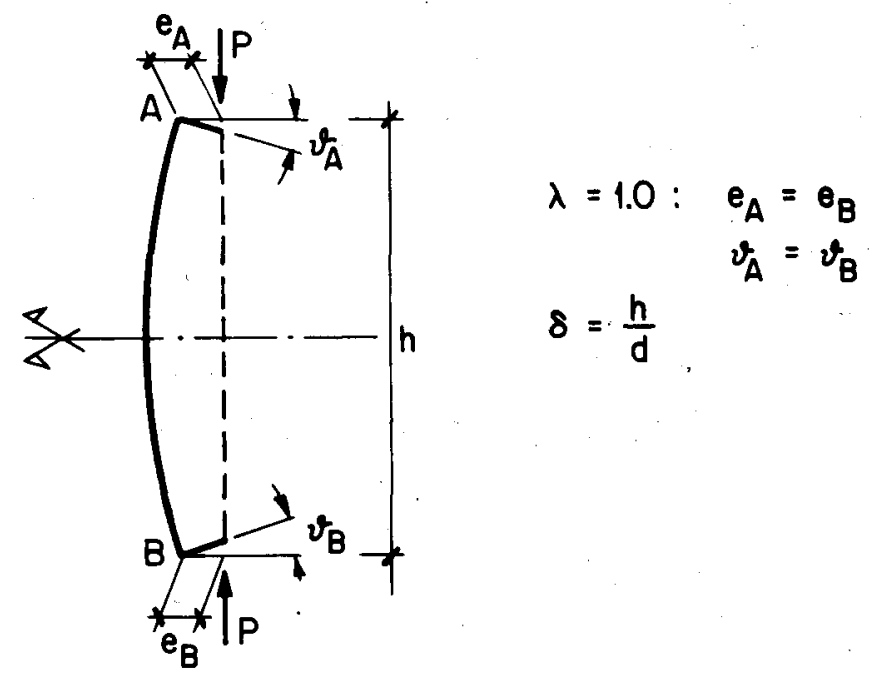

\section{Bild 6.6: Symmetrischer Belasłungsfall}

Die obigen Beziehungen werden nun für den symmetrischen Belastungsfall in Bild 6.6 dargestellt. Die Wand ist durch die Linienlast $P$ axial belastet. Die Exzentrizitäten $e_{A}$ und $e_{B}$ sowie die Wandendverdrehungen $\vartheta_{A}$ und $\vartheta_{B}$ sind gleich gross. $\lambda$ beträgt demnach 1 . Eie Axiallast $P$ ist auf die Bruttoquerschnittsfläche $d \cdot 1$ bezogen $\left(\sigma_{m}=P / d\right)$ und die nachfolgenden Beziehungen zwischen $\sigma_{m}$, $e_{A}$, $\vartheta_{A}$ und $\delta$ sind mit der Bezugsspannung $\sigma_{b}$ bzw. Bezugsstauchung $\varepsilon_{b}$ normiert (vgl. 4.1):

$$
\begin{aligned}
& \frac{{ }^{e} A}{d}=\frac{1}{6} \cdot \cos \left(\frac{\delta}{2} \cdot \sqrt{12 \cdot \frac{\sigma_{m}}{\sigma_{b}} \cdot \varepsilon_{b}}\right) \cdot\left(\frac{\sigma_{F} \cdot \sigma_{b}}{\sigma_{b} \cdot \sigma_{m}}-1\right) \\
& \frac{\vartheta_{A}}{\sqrt{\varepsilon_{b}}}=\frac{e_{A}}{d} \cdot 12 \cdot \frac{\sigma_{m}}{\sigma_{b}} \cdot\left\{\frac{1-\cos \left(\delta \cdot \sqrt{12 \cdot \frac{\sigma_{m}}{\sigma_{b}} \cdot \varepsilon_{b}}\right)}{\sin \left(\delta \cdot \sqrt{12 \cdot \frac{\sigma_{m}}{\sigma_{b}} \cdot \varepsilon_{b}}\right)}\right\}
\end{aligned}
$$

\section{Beanspruchungsart E}

Bei konstantem Exzentrizitätsmass $m_{A}$ wächst die Wandendverdrehung ${ }_{A}$ überproportional $z u \sigma_{m}$ an. In Bild 6.7 sind solche Kurven für verschiedene $m_{A}{ }^{-}$ Werte dargestellt. $\varepsilon_{b}$ beträgt 0.002 . Die Kurven steigen unabhängig von $m_{A}$ und $\delta$ monoton an und nähern sich asymptotisch der kritischen Spannung $\sigma_{k r}$. Ein Stabilitätsversagen tritt demnach nur bei zentrischer Last $\left(m_{A}=0\right)$ auf. Wird $\sigma_{m}$ auf $\sigma_{k r}$ bezogen, so verdeutlicht, sich dieser Zusammenhang (Bild 6.B). 


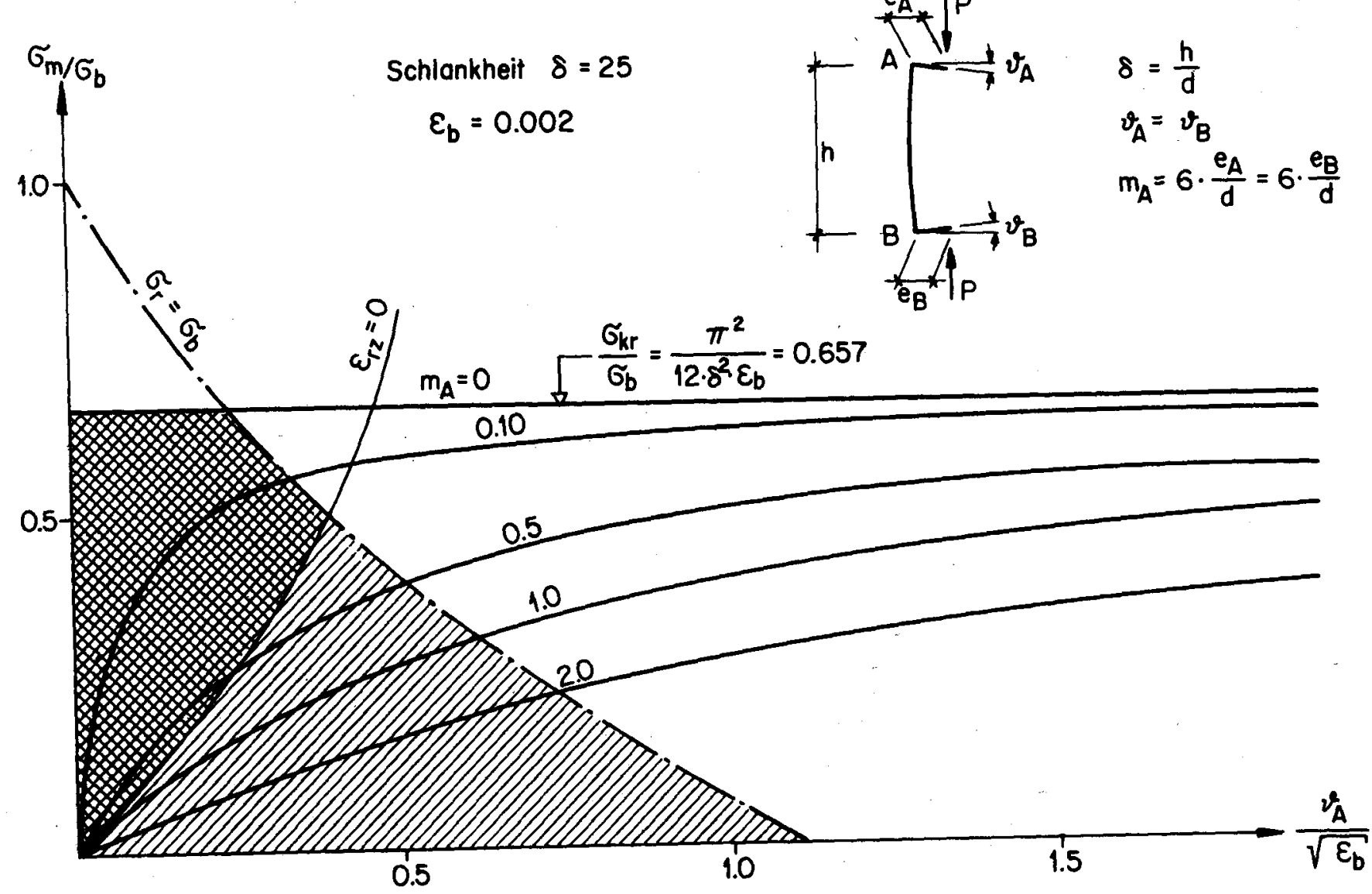

Bild 6.7: $\sigma_{m}-v_{A}\left(m_{A}=\right.$ konst $)$

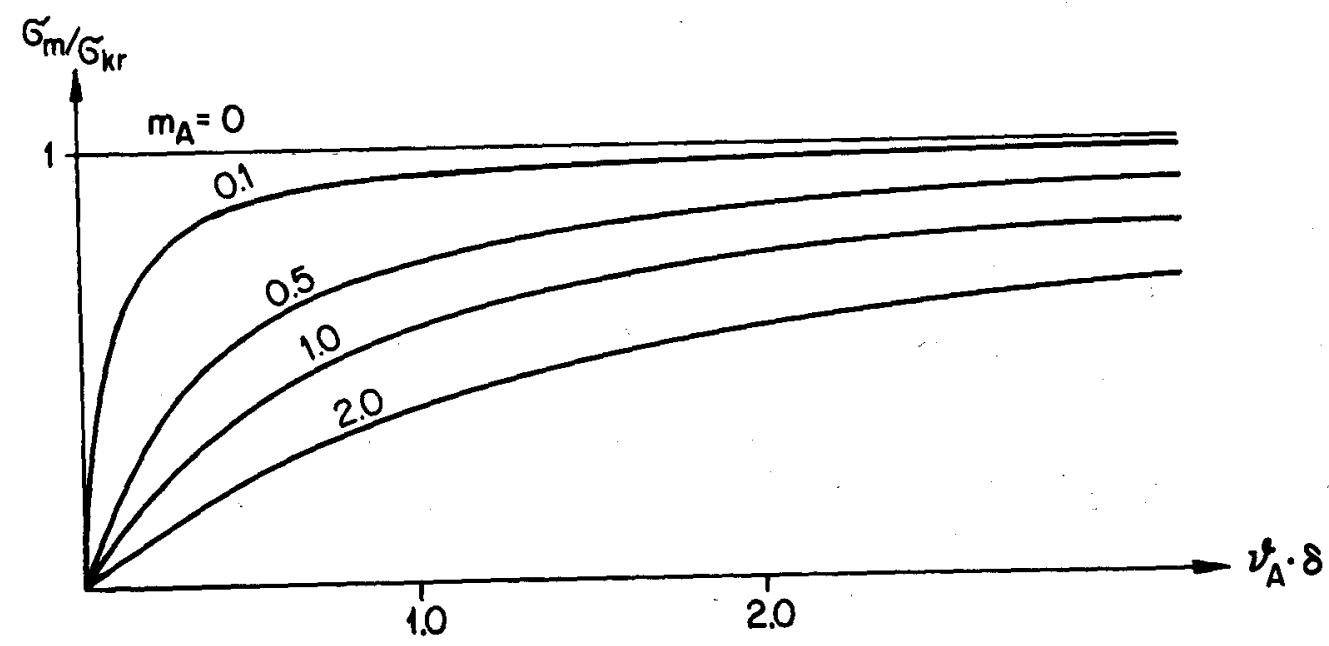

Bild 6.8: $\sigma_{m}-v_{A}\left(m_{A}=\right.$ konst $)$ 
Bisher wurde ein linear elastisches Material mit unbegrenzter Zug- und Druckfestigkeit vorausgesetzt. Es wird nun angenommen, dass die Bezugsspannung $\sigma_{b}$ der Mauerwerksfestigkeit $\sigma_{u}$ entspricht $\left(\sigma_{b}=\sigma_{u}\right)$. Dementsprechend ist $\varepsilon_{b}$ gleich der Bruchstauchung $\varepsilon_{u}$. Bezüglich der Materialzugfestigkeit werden keine Einschränkungen gemacht.

$\sigma_{u}$ tritt zuerst in der Randfaser des meistbeanspruchten Querschnittes in Wandmitte auf. In Bild 6.7 ist die Kurve konstanter Randdruckspannung $\left(\sigma_{r}=\sigma_{b}\right)$ strichpunktiert dargestellt. Bei verschwindender Verdrehung läuft diese Kurve durch Punkt $(0 ; 1)$ und fällt mit zunehmender Verdrehung $\vartheta_{A}$. Sie begrenzt das schraffierte Gebiet.

Entlang der Kurve $\varepsilon_{r z}=0$ beträgt das Exzentrizitätsmass $m$ im meistbeanspruchten Querschnitt in Wandmitte 1; es treten also in jeder Faser der Wand Druckspannungen auf. Diese Kurve tangiert im Ursprung die Kurve $m=1$. Sie unterteilt das schraffierte Gebiet zusätzlich: Im stark schraffierten Gebiet treten somit in jedem Querschnitt der Wand nur Druckspannungen auf, die $\sigma_{b} b z w$. $\sigma_{u}$ nicht übersteigen.

\section{Beanspruchungsart $V$}

Bei der Beanspruchungsart $V$ werden der Wand durch die darüber und darunterliegende Decke Verdrehungen der Grösse $\vartheta_{A}$ und $\vartheta_{B}$ aufgezwungen. Beim symmetrischen Belastungsfall (Bild 6.6) sind $\vartheta_{A}$ und $\vartheta_{B}$ entgegengesetzt gleich gross. Werden nun der Wand unter konstanter Axiallast $P$ bzw. Spannung $\sigma_{m} / \sigma_{b}$ Verdrehungen aufgezwungen, so ergibt sich zwischen dem dabei auftretenden Exzentrizitätsmass $m_{A}$ und der Verdrehung $\vartheta_{A}$ ein linearer Zusammenhang (Bild 6.9). Je grösser $\sigma_{m} / \sigma_{b}$, desto flacher verläuft die Gerade. Die Schlankheit $\delta$ ist Parameter der Geradenschar in Bild 6.9. Sie beträgt 25. Bei $\sigma_{m} / \sigma_{b}=0.657$ verläuft die Gerade horizontal. Die Verdrehung ist damit unabhängig vom Exzentrizitätsmass $m_{A}$. Es handelt sich dabei um die kritische Spannung.

Aus der bekannten Formel für die Eulerlast

$P_{k r}=\frac{\pi^{2} \cdot E I}{h^{2}}$

ergibt sich

$\frac{\sigma_{k r}}{\sigma_{b}}=\frac{\pi^{2}}{12 \cdot \delta^{2} \cdot \varepsilon_{b}}$

un nach Einsetzen von $\delta=25$ und $\varepsilon_{b}=0.002$

$\frac{\sigma_{k r}}{\sigma_{b}}=0.657$ 


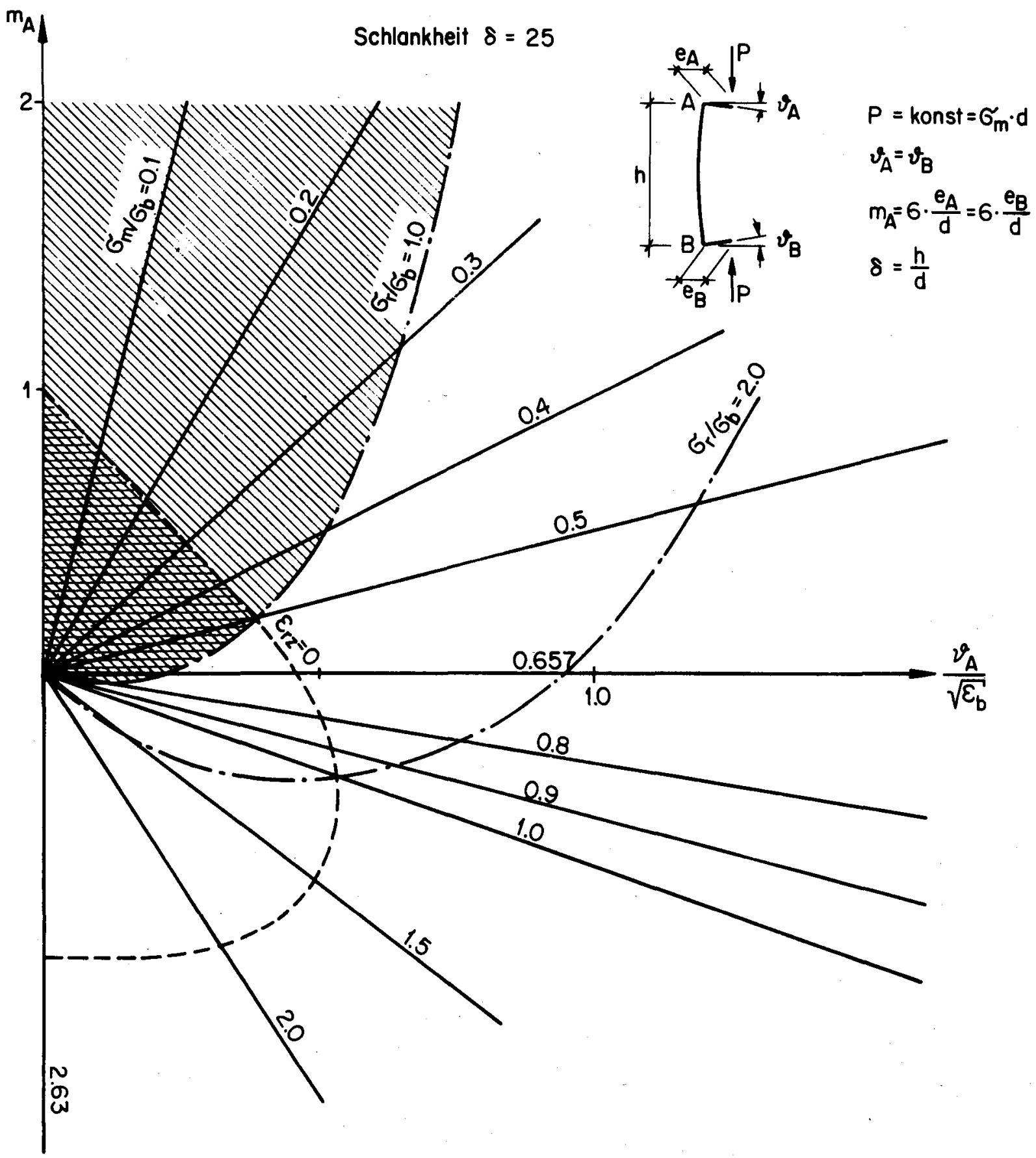

Bild 6.9: $m_{A}-\nu_{A}\left(\sigma_{m} / \sigma_{b}=\right.$ konst $)$

Bei der Beanspruchungsart $E$ ist die Tragfähigkeit bei der Eulerlast erreicht. Im vorliegenden Fall jedoch vermag die Wand höhere Lasten aufzunehmen. Für $\sigma_{m} / \sigma_{b} \geq 0.657$ liegen die $m_{A} \vartheta_{A}^{-G e r a d e n}$ im vierten Quadranten. Das Exzentrizitätsmass $m_{A}$ ist damit negativ, d.h. die Wand stützt sich auf die darunterund darüberliegende Decke ab. Die Decken ihrerseits erfahren dadurch eine negative Einspannung. Die Tragfähigkeit der Wand ist dann erreicht, wenn die Last der vierfachen Eulerlast entspricht bzw. wenn $\sigma_{m} / \sigma_{b}=2.6316$. Die Knicklänge entspricht dann gerade der halben Wandhöhe. In Bild 6.10 sind die dazugehörigen Verläufe der Wandaxe dargestellt. 


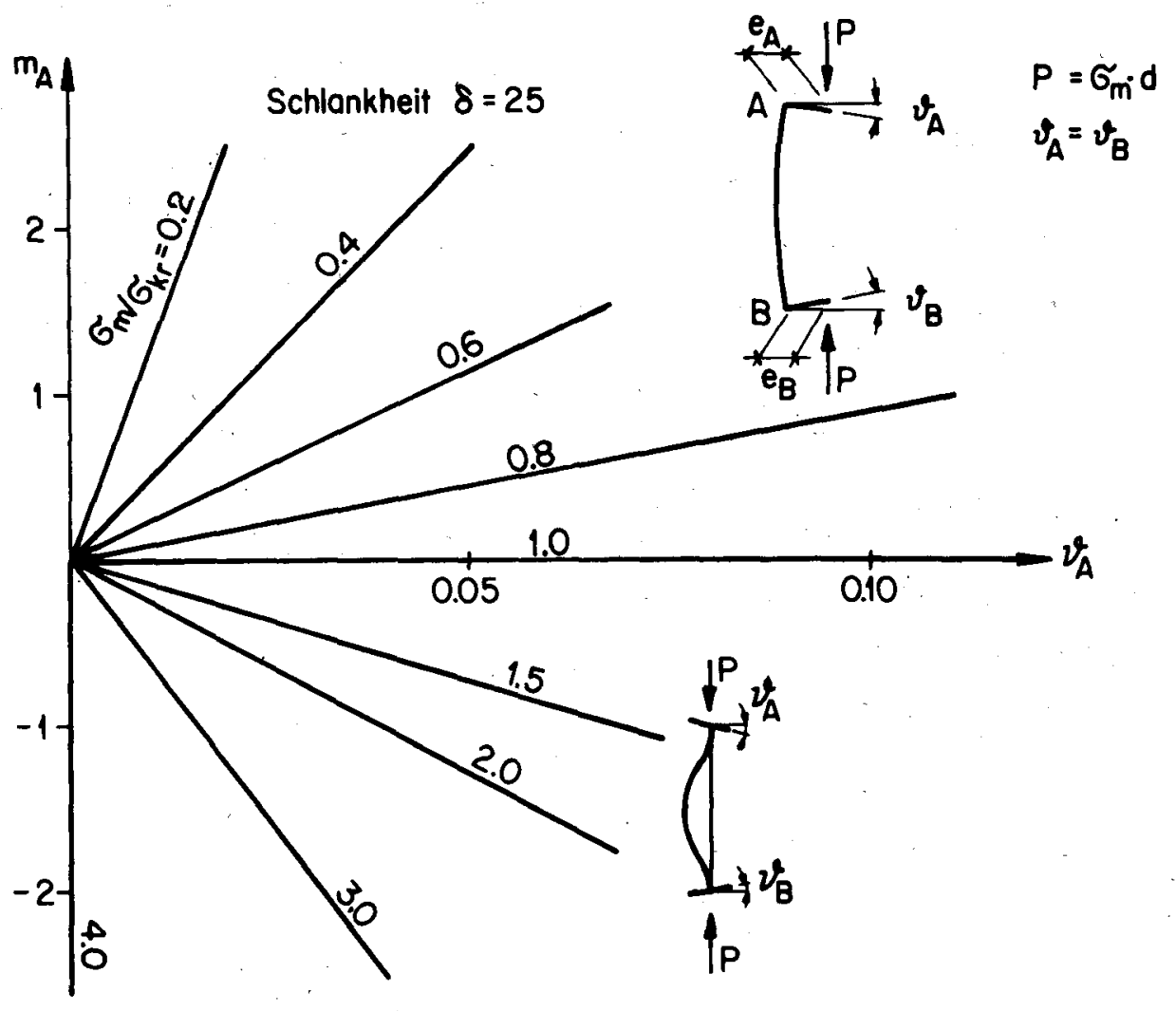

Bild 6.10: $m_{A}-v_{A}\left(\sigma_{m} / \sigma_{k r}=\right.$ konst $)$

Es wird nun wiederum angenommen, dass $\sigma_{b}$ der Mauerwerksfestigkeit $\sigma_{u}$ entspricht. Der Gültigkeitsbereich der Geraden in Bild 6.9 wird dadurch durch die Kurve konstanter, maximaler Randspannung $\sigma_{r} / \sigma_{b}=\sigma_{r} / \sigma_{u}=1$ auf das schraffierte Gebiet begrenzt. Entlang der gestrichelten Kurve beträgt das Exzentrizitätsmass $m$ im meistbeanspruchten Querschnitt in Wandmitte 1. Die eine Randfaser in diesem Querschnitt ist also spannungsfrei, in allen anderen Fasern der Wand treten aber Druckspannungen auf. Das schraffierte Gebiet kann damit weiter unterteilt werden: Im stark schraffierten Gebiet sind in keinem Wandquerschnitt Zugspannungen vorhanden.

Der Zusammenhang zwischen der Last bzw. der Spannung und der dabei möglichen Verdrehung bis zum Bruch $\left(\sigma_{r} / \sigma_{b}=\sigma_{r} / \sigma_{u}=1\right)$ geht deutlicher aus Bild 6.11 hervor. Diese Spannung wird als Traglastspannung bezeichnet. Als Traglast wird die maximale Last, der die Wand widerstehen kann, verstanden. Für Schlankheiten $\delta$ von 25 und 50 sind entsprechende Bruchkurven dargestellt. Für $\delta=25$ umschliesst diese das schraffierte Gebiet. Wie in Bild 6.9 ist dieses weiter unterteilt: Im stärker schraffierten Bereich treten zudem in keinem Wandquerschnitt Zugspannungen auf; alle Fasern sind gestaucht. 


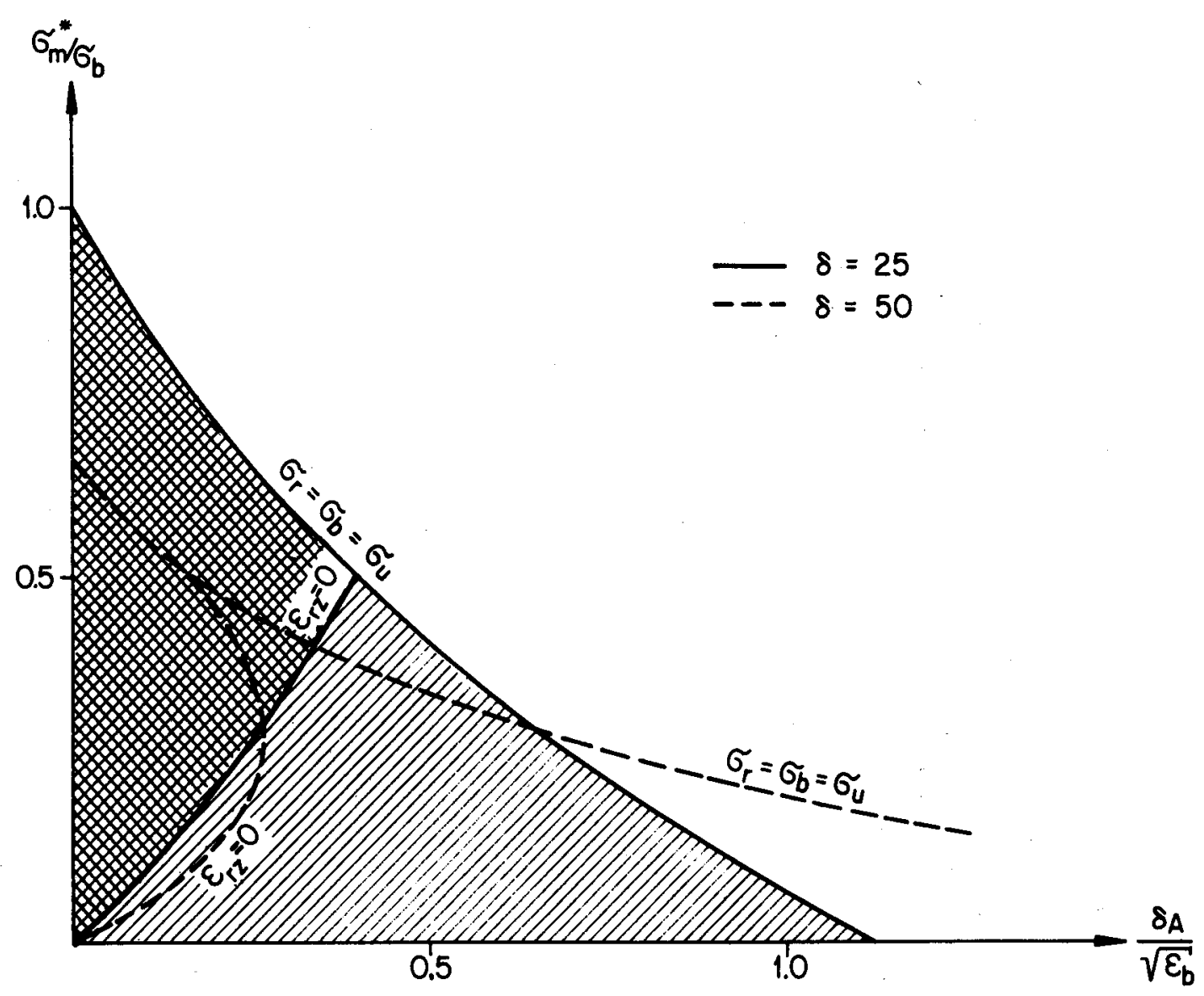

Bild 6.11: $\sigma^{*}-v_{A}\left(\sigma_{r}=\sigma_{b}=\sigma_{u} ; \varepsilon_{r z}=0\right)$

\subsubsection{Linear elastisches Material ohne Zugfestigkeit}

Bei fehlender Zugfestigkeit ergeben sich bei Exzentrizitäten, die die Kernweite übersteigen, im Exzentrizitäts-Krümmungs-Verhalten Abweichungen gegenüber einem Material mit Zugfestigkeit (vgl. Kapitel 4).

Die exzentrisch belastete Wand in Bild 6.4 ist demnach in zwei Zonen zu unterteilen: Im ungerissenen Bereich übersteigt die Exzentrizität die Kernweite nicht. In der gerissenen Zone liegt die Axiallast ausserhalb des Kerns (Bild 6.12).

Im ungerissenen Bereich gilt für den Zusammenhang zwischen der Krümmung und dem Moment die Beziehung 6.4. Für den gerissenen Bereich kann die Beziehung aus der Darstellung in Bild 6.13 folgendermassen hergeleitet werden:

Bei der dreieckförmigen Spannungsverteilung beträgt die Randstauchung

$$
\varepsilon_{r}=\frac{2 \cdot P}{E \cdot \eta}
$$




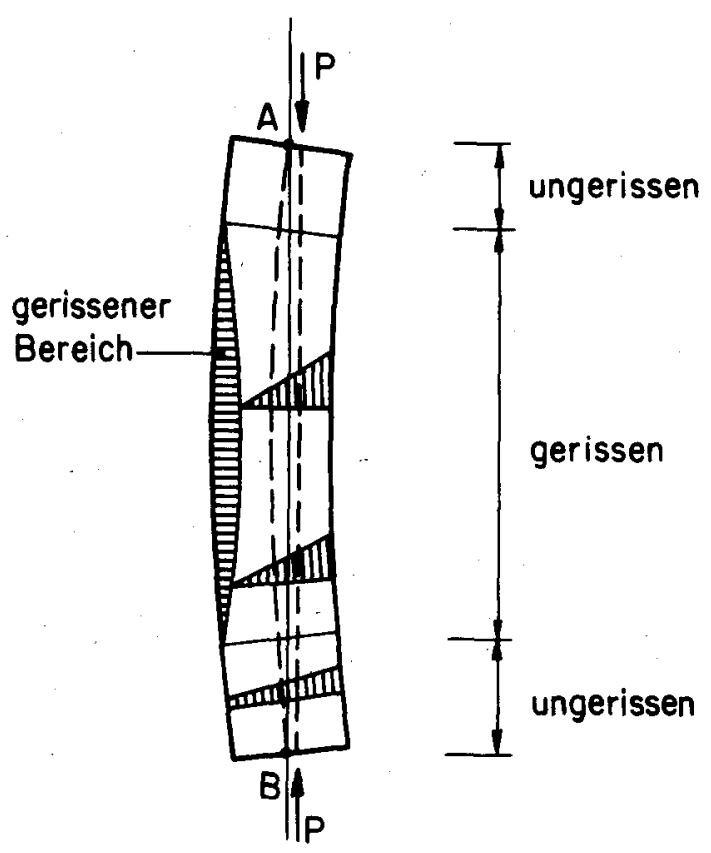

Bild 6.12 : Ungerissener-gerissener Bereich

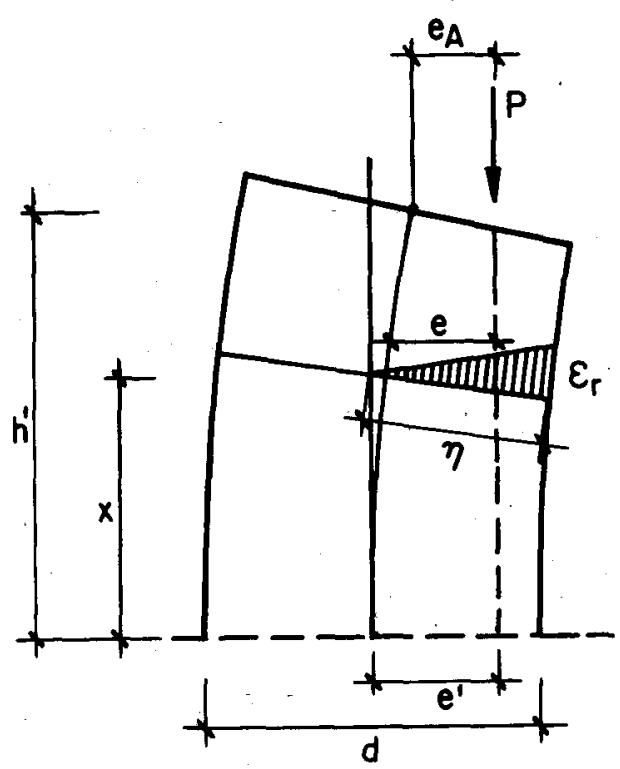

Bild 6.13: Bezeichnungen

Der Abstand der Neutralaxe vom Querschnittsrand beträgt $\eta$. Aus einer geometrischen Betrachtung ergibt sich zwischen $n$ und e folgender Zusammenhang

$$
n=3 \cdot\left(\frac{d}{2}-e\right)
$$

Die Krümmung der Wandaxe kann aus $\varepsilon_{r}$ und $n$ wie folgt bestimmt werden

$$
\frac{d^{2} \cdot e}{d x^{2}}=\frac{\varepsilon_{r}}{n}=\frac{2 \cdot p}{g \cdot E \cdot\left(\frac{d}{2}-e\right)^{2}}
$$


Diese Differentialgleichung (6.16) wurde von Angervo 1954 [2] gelöst.

Für den Wandabschnitt in Bild 6.13 ergeben sich nach Einführung vier Grössen $p$ und $m_{A}$ folgende Beziehungen:

$$
\begin{array}{lr}
e^{\prime}=\frac{d}{6}+p \cdot \frac{d}{3} & -0.5 \leq p \leq 1 \\
m_{A}=\frac{6 \cdot e_{A}}{d} & 0 \leq m_{A} \leq 3
\end{array}
$$

a) Innerhalb des ganzen Wandabschnittes liegt die Wirkungslinie der Axiallast $p$ ausserhalb des Kerns: Für $m$ und $p$ ergeben sich demnach folgende Definitionsbereiche:

$$
\begin{aligned}
1 & \leq m_{A} \leq 3 \\
\frac{m_{A}-1}{2} & \leq P<1
\end{aligned}
$$

Die Wandendverdrehung an der Stelle $x=h$ ' bzw. $e=e_{A}$ beträgt:

$$
\left.\frac{d e}{d x}\right|_{e=e_{A}}=\mp \frac{d}{3} \cdot \sqrt{\frac{P}{E I}} \cdot \sqrt{\frac{1+2 \cdot P-m_{A}}{(1-p) \cdot\left(3-m_{A}\right)}}
$$

und für h' kann folgende Beziehung angegeben werden:

$$
\left.\begin{array}{rl}
h^{\prime} \cdot \sqrt{\frac{P}{E I}}= & \frac{1}{2} \cdot \sqrt{(1-p) \cdot\left(3-m_{A}\right) \cdot\left(1+2 \cdot p-m_{A}\right)}+\frac{1}{2} \cdot(1-p)^{3 / 2} \\
& \cdot \ln \left\{\frac{\left(2-m_{A}+p\right) \cdot \sqrt{\left(3-m_{A}\right) \cdot\left(1+2 \cdot p-m_{A}\right)}}{1-p}\right\}=f_{1}\left(p, m_{A}\right)
\end{array}\right\}
$$

b) Die Exzentrizität übersteigt die Kernweite nur im unteren Wandbereich von Bild 6.13. Die Definitionsbereiche für $m$ und $p$ lauten dann:

$$
\begin{aligned}
& \left.\begin{array}{l}
0<m_{A}<1 \\
0<p<1
\end{array}\right\} \\
& \left.\frac{d e}{d x}\right|_{E=e_{A}}=\mp \frac{d}{6} \cdot \sqrt{\frac{P}{E I}} \cdot \sqrt{\frac{1+3 \cdot P}{1-p}-m_{A}^{2}} \\
& h^{\prime} \cdot \sqrt{\frac{P}{E I}}=p \cdot(1-p)+\frac{1}{2} \cdot(1-p)^{3 / 2} \cdot \ln \left(\frac{1+\sqrt{P}}{1-\sqrt{P}}\right)+ \\
& \arcsin \sqrt{\frac{1-p}{1+3 \cdot p}}-\arcsin \left(m_{A} \cdot \frac{1-p}{1+3 \cdot p}\right)=f_{2}\left(p, m_{A}\right)
\end{aligned}
$$


c) Die Axiallast $P$ liegt im ganzen Wandabschnitt innerhalb des Kerns. Für $P$ und $m_{A}$ ergeben sich folgende Definitionsbereiche:

$$
\left.\begin{array}{rl}
0 & \leq m_{A} \leq 1 \\
\frac{m_{A}-1}{2} & \leq p<0
\end{array}\right\}
$$

Die Wandendverdrehung sowie die Beziehung für h können folgendermassen angegeben werden:

$$
\begin{aligned}
& \left.\frac{d e}{d x}\right|_{B=e_{A}}=\mp \frac{d}{G} \cdot \sqrt{\frac{P}{E I}} \cdot \sqrt{(1+2 \cdot p)^{2}-m_{A}^{2}} \\
& h^{\prime} \cdot \sqrt{\frac{P}{E I}}=\frac{\pi}{2}-\arcsin \left(\frac{m_{A}}{1+2 \cdot p}\right)=f_{3}\left(p, m_{A}\right)
\end{aligned}
$$

Die angegebenen Beziehungen gelten für den Wandabschnitt von Bild 6.13. e' ist dabei stets die grösste Exzentrizität im Wandabschnitt der Höhe $h$ und an der Stelle $x=0$ ist $d e / d x=0$. Jede beliebig belastete Wand der Höhe $h$ kann nun in einzelne derartige Wandabschnitte unterteilt werden. Am Beispiel des symmetrischen Belastungsfalles von Bild 6.6 soll das Vorgehen erläutert werden.

\section{2 .2 .1 Symmetrische Wandbelastung}

In Bild 6.14 a ist wiederum die exzentrisch belastete Wand von Bild 6.6 dargestellt. Die Wand der Höhe h wird durch zwei gleiche Wandabschnitte der Höhe h' gebildet. Die einzelnen Teile entsprechen dabei genau dem Wandabschnitt in Bild 6.13. Anstelle von h' ist demnach h/2 einzusetzen. Die Exzentrizität $e_{A}$ entspricht e. Es gilt:

$$
\begin{array}{ll}
h & =2 \cdot \sqrt{\frac{E T}{P}} \cdot f_{i}\left(p, m_{A}\right) ; \\
m_{A} & =\frac{6 \cdot e_{A}}{d}
\end{array}
$$

Die Wandverdrehung $\vartheta_{A}$ ist durch die Beziehungen (6.18), (6.21) oder (6.24) gegeben. Neben der verformten Lage der Wandaxe von Bild 6.14 a ist auch die Ausbiegungskurve in Bild 6.14 b denkbar. Die Wandhöhe hergibt sich dann durch eine Kombination von einzelnen Wandabschnitten:

$$
h=2 \cdot \sqrt{\frac{E T}{P}} \cdot\left\{2 \cdot f_{i}(p, 0)-f_{i}\left(p, m_{A}\right)\right\} \quad ; i=1,2,3
$$



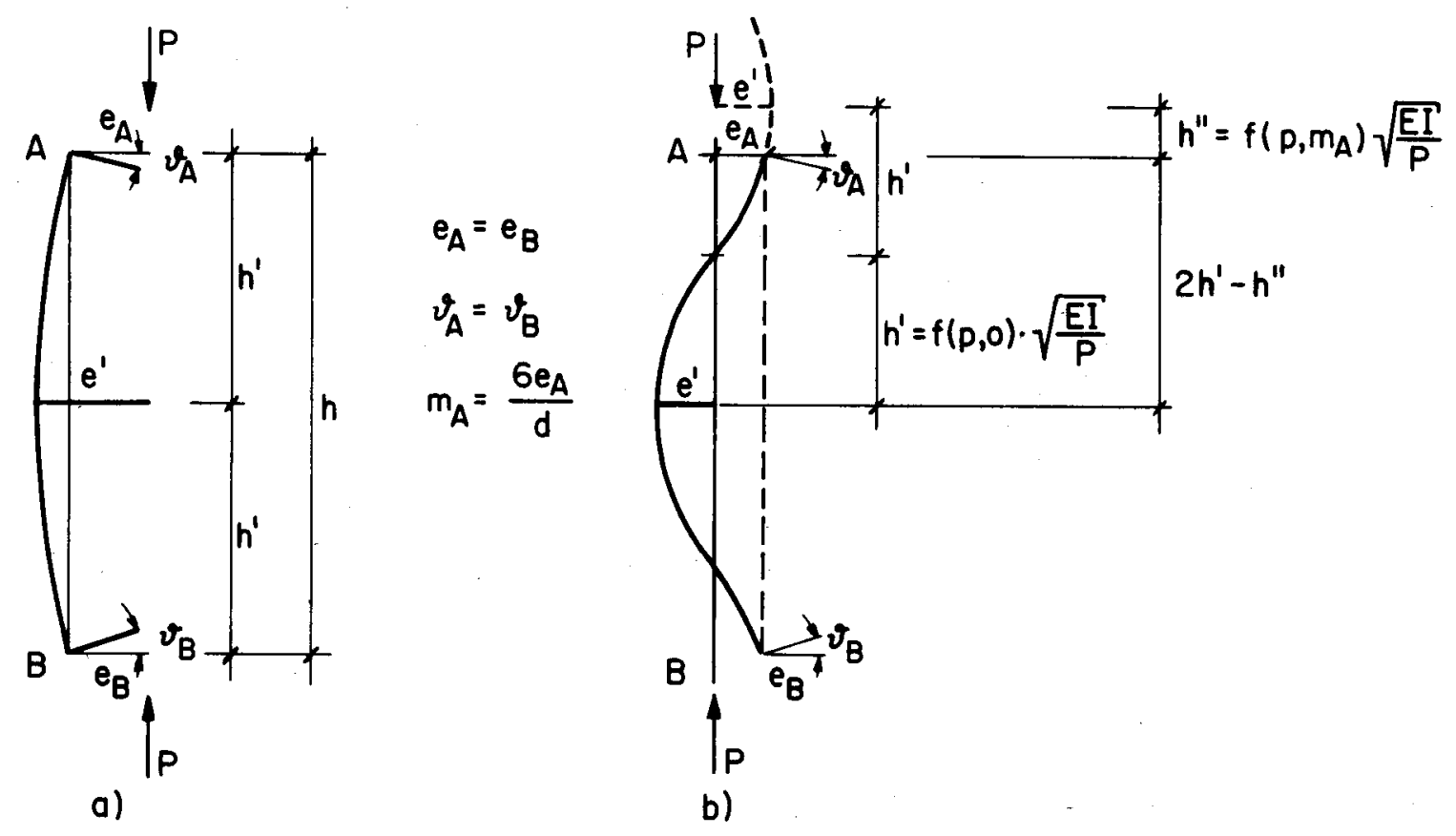

Bild 6.14: Symmetrische Wandbelastung

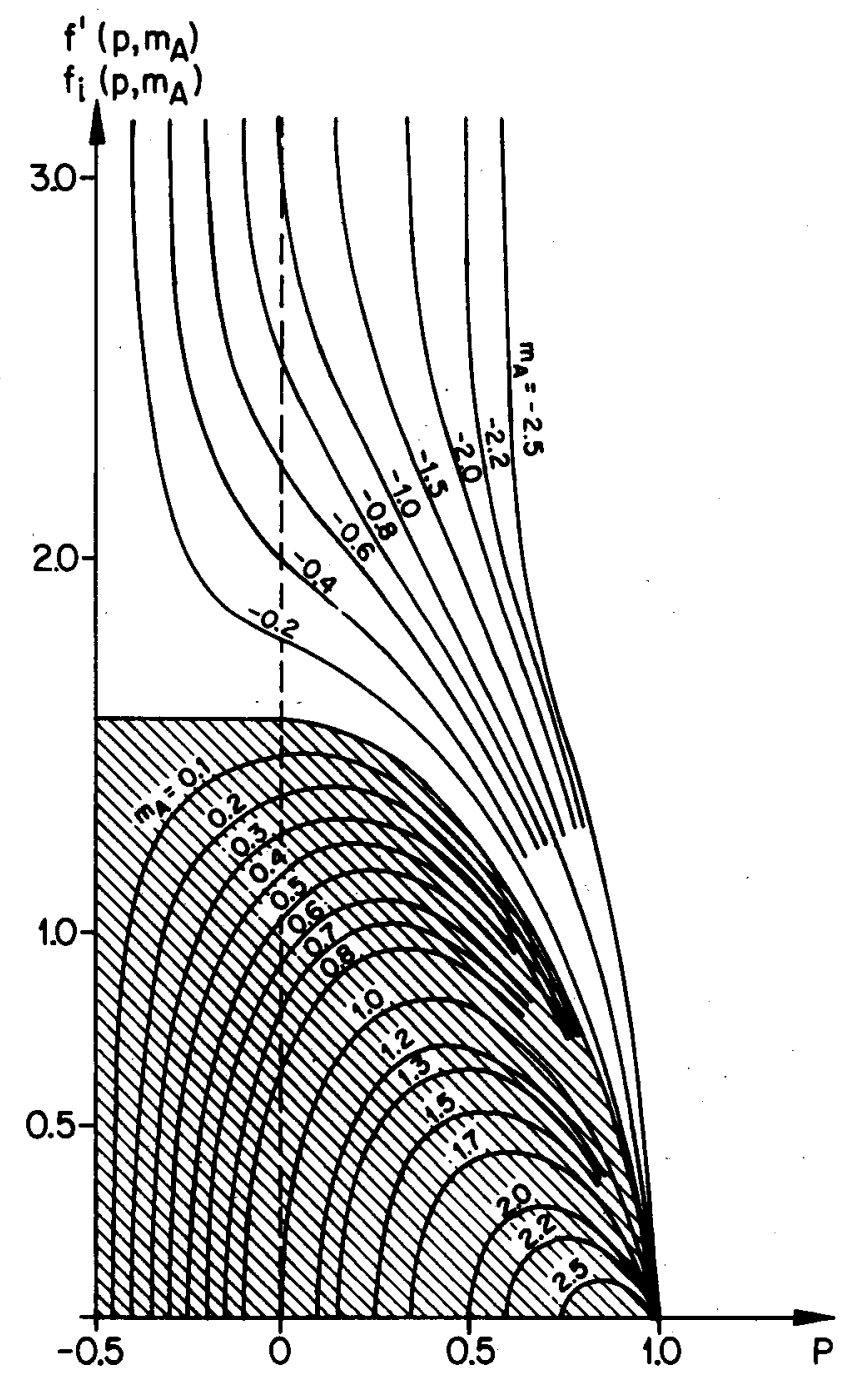

Bild 6.15: Funktionen $f_{i}\left(p, m_{A}\right), f^{\prime}\left(p, m_{A}\right)$ 
Gemeinsame Basis für alle Wandabschnitte ist dabei die maximale Exzentrizität $e^{\prime}$. Die Wandendverdrehung $\vartheta_{A}$ ist wiederum durch die Beziehungen (6.1B), (6.21) oder (6.24) gegeben, wobei für $m_{A}$ der Wert $m_{A}=6 \cdot e_{A} / d$ einzusetzen ist. Die Funktion $f_{i}\left(p, m_{A}\right)$ von $G l$. (6.26) und die Funktion $f^{\prime}\left(p, m_{A}\right)=$ $2 \cdot f_{i}(p, 0)-f_{i}\left(p, m_{A}\right)$ von GI. (6.27) sind in Abhängigkeit von $p$ und $m_{A}$ in $B i l d$ 6.15 dargestellt. Im schraffierten Gebiet liegt der Wertebereich von $f_{i}\left(p, m_{A}\right)$ und im nicht schraffierten Gebiet derjenige von $f^{\prime}\left(p, m_{A}\right)$.

\section{Beanspruchungsart E}

Die Wand in Bild 6.14 a werde nun bei konstantem Exzentrizitätsmass $m_{A}=$ 6. ${ }_{A} / d$ zunehmend belastet. Die entsprechenden Last-Verdrehungs-Kurven sind in Bild 6.16 dargestellt. Anstelle der Last $P$ wurde die mittlere Spannung $\sigma_{m}$ eingeführt. Das Exzentrizitätsmass $m_{A}$ ist Kurvenparameter und die Schlankheit $\delta$ beträgt 25 .

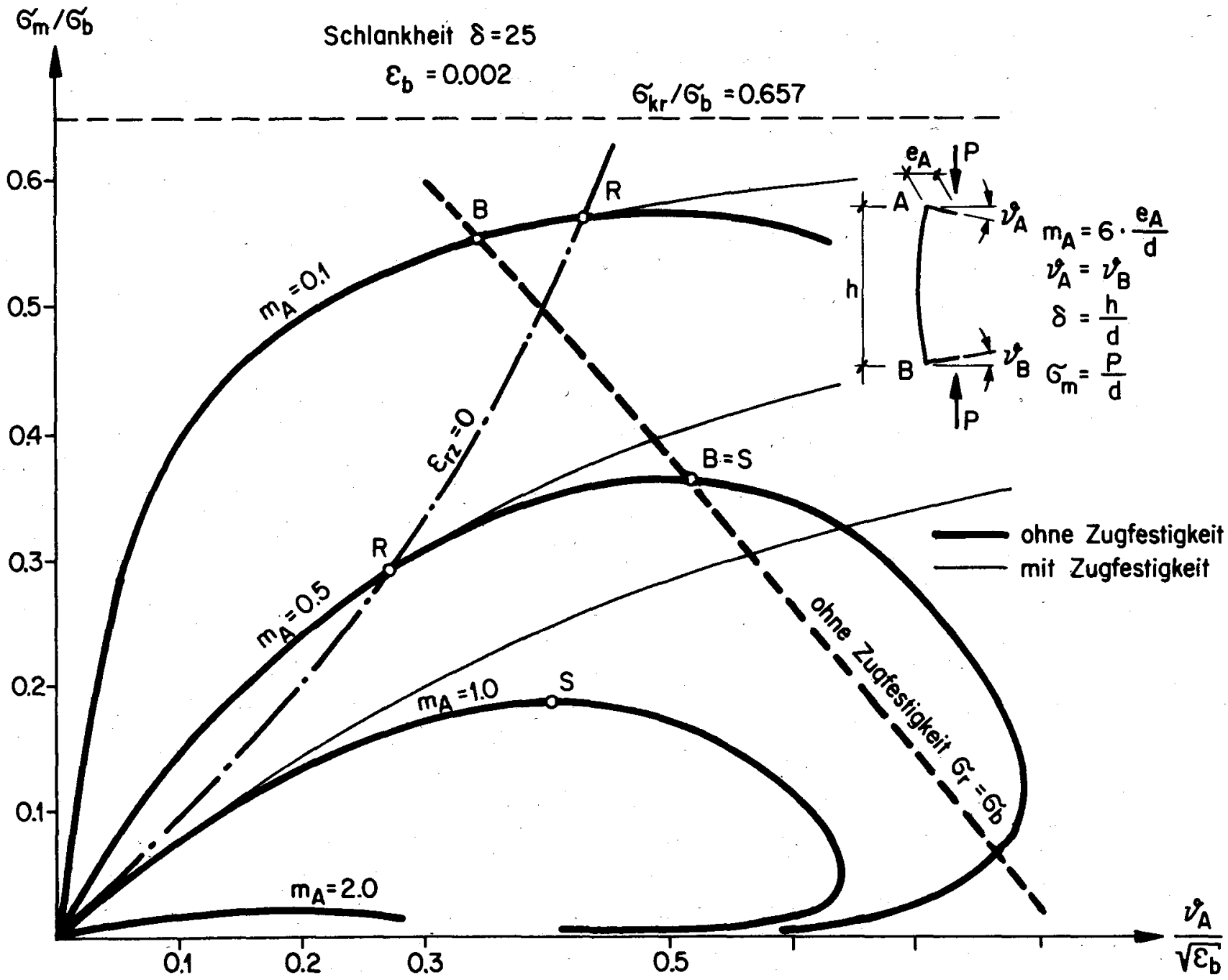

Bild 6.16: $\sigma_{m}-\nu_{A}\left(m_{A}=\right.$ konst. $)$ 
Ausser diesen stark ausgezogenen Kurven sind noch zwei weitere von Bedeutung: Für alle Kombinationen von $\sigma_{m}$ und $\vartheta_{A}$ auf der gestrichelten Kurve ist die Randdruckspannung $\sigma_{r}$ im meistbeanspruchten Querschnitt gleich der Bezugsspannung $\sigma_{b}$ und für solche auf der strichpunktierten Kurve ist das Exzentrizitätsmass $m$ im selben Querschnitt 1 bzw. die Randzugdehnung $\varepsilon_{r z}$ gleich null.

Diese beiden Kurven werden von $\sigma_{m}{ }^{-\vartheta} A$-Kurven geschnitten. Unter der Annahme, dass die Bezugsspannung $\sigma_{b}$ wiederum der Mauerwerksfestigkeit $\sigma_{u}$ gleichgesetzt wird, erfolgt der Bruch (Punkt $B$ ) bei kleinem Exzentrizitätsmass $\left(m_{A}=0.1\right)$ vor dem Auftreten von Randdehnungen (Punkt $R$ ). Letztere entsprechen bei einem Material ohne Zugfestigkeit spezifischen Rissöffnungen. Bei grösserem Exzentrizitätsmass $\left(m_{A}=0.5\right)$ ergeben sich solche in Punkt $R$ vor dem Bruch (Punkt $B$ ).

Für Werte $m_{A} \geq 1$ treten bereits bei sehr kleinen $\vartheta_{A}$ bzw. $\sigma_{m}$ spezifische Rissöffnungen auf. Im Spannungsgipfel, d.h. im Punkt mit horizontaler Tangente an die $\sigma_{m}{ }^{-\vartheta}$-Kurve tritt ein Stabilitätsversagen ein (Punkt $s$ ). Bei $m_{A}=0.5$ fallen Stabilitätsversagen und Materialversagen $\left(\sigma_{r}=\sigma_{b}=\sigma_{u}\right)$ zusammen (Punkt $S=$ Punkt $B$ ).

Die Abweichungen im Tragverhalten gegenüber einem gleichen Material mit Zugfestigkeit sind ebenfalls aus Bild 6.16 erkennbar. Bis zum ersten Auftreten von Randdehnungen im Querschnitt in Wandmitte (strichpunktierte Kurve) sind naturgemäss keine Unterschiede vorhanden. Nach dem Ueberschreiten dieser Grenze weichen die Kurven in zunehmendem Masse voneinander ab. Die schwach ausgezogenen Kurven (mit Zugfestigkeit) steigen monoton weiter an und nähern sich asymptotisch der kritischen Spannung $\sigma_{k r}$. Im Gegensatz zum zugfesten, linear elastischen Material tritt bei linear elastischem Material ohne Zugfestigkeit nicht nur bei zentrischer Last $\left(m_{A}=0\right)$ Stabilitätsversagen auf.

Die Traglast $P^{*}$, wiederum definiert als die maximale Last, der die Wand widerstehen kann, ist je nach Exzentrizitätsmass $m_{A}$ durch Stabilitäts- oder Materialversagen $\left(\sigma_{r}=\sigma_{b}=\sigma_{u}\right)$ bestimmt.

In Bild 6.17 ist die Traglastspannung $\sigma^{*} / \sigma_{b}$ in Abhängigkeit der Schlankheit $\delta$ aufgetragen. Das Exzentrizitätsmass $m_{A}$ ist Kurvenparameter. Im schraffierten Bereich ist $\sigma^{*}$ durch Stabilitätsversagen, im nicht schraffierten durch Materialversagen bestimmt. Die Punkte $B\left(m_{A}=0.1\right), B=S\left(m_{A} \cdot 1.0\right)$ und $S\left(m_{A}=1.0\right)$ von Bild 6.16 wurden übertragen. 


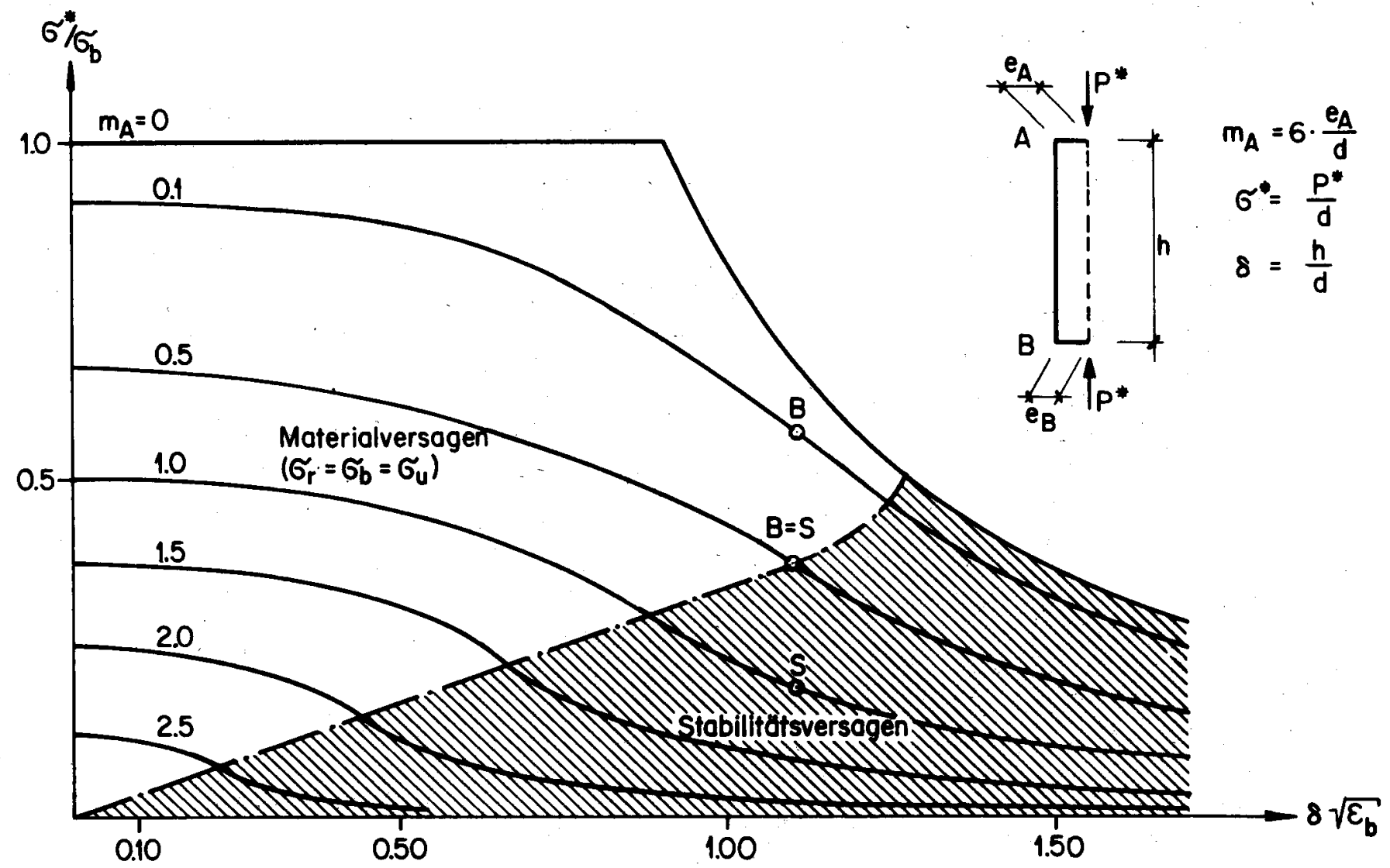

Bild 6.17: $\sigma^{*}-\delta\left(m_{A}=\right.$ konst. $)$ 


\section{Beanseruchungsart_B}

In Bild 6.18 ist das Exzentrizitätsmass $m_{A}$ als Funktion der Wandverdrehung $\vartheta_{A}$ dargestellt. Entlang den Kurven ist die mittlere Spannung $\sigma_{m} / \sigma_{b}$ konstant $\left(\sigma_{m} / \sigma_{b}=.001,0.1,0.3\right.$ und 0.5$)$. Die Schlankheit $\delta$ beträgt 25 .
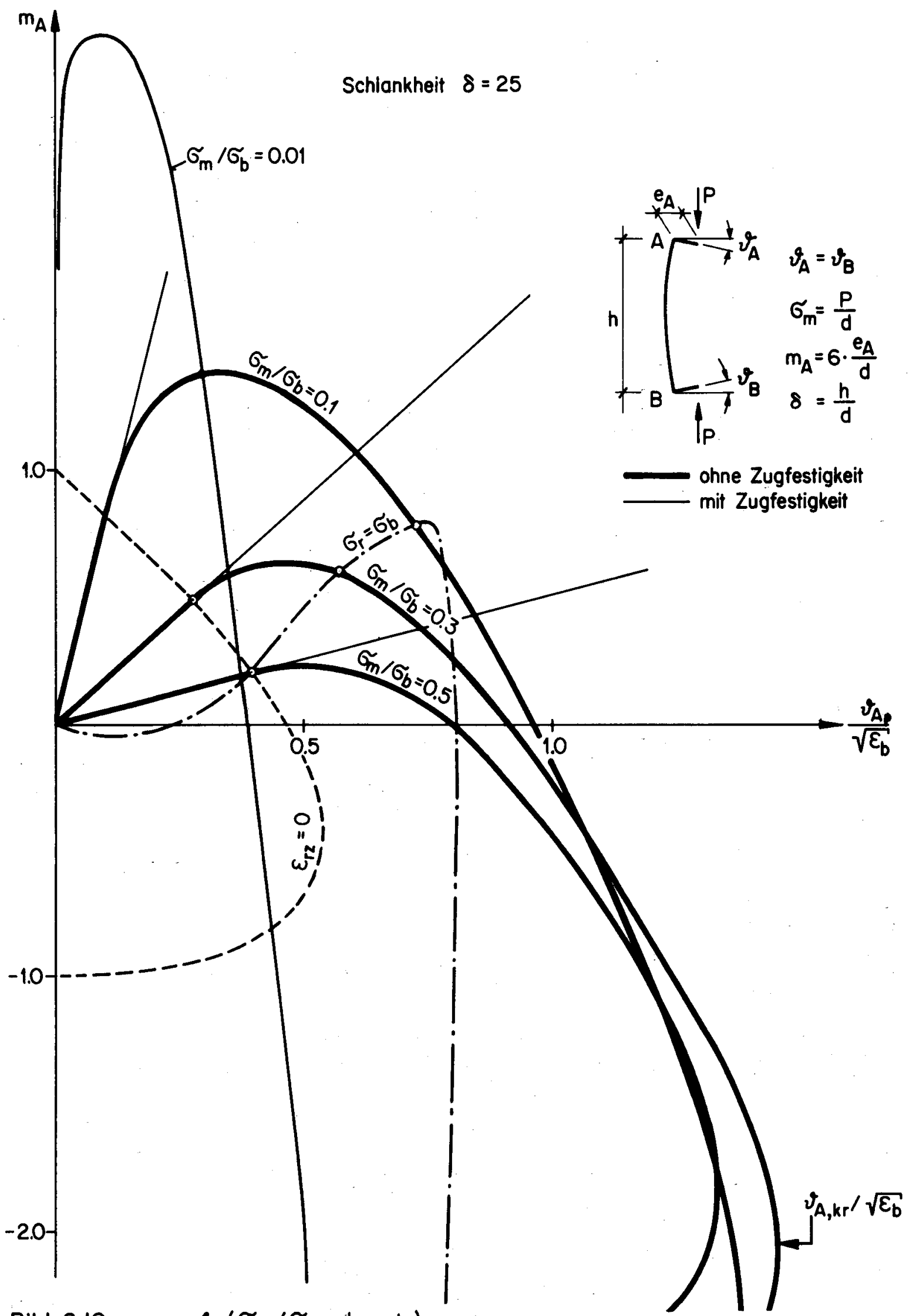

Bild $6.18: m_{A}-v_{A}\left(\sigma_{m} / \sigma_{b}=\right.$ konst. $)$ 
Die Kurven verlaufen zunächst linear. Beim Auftreten von Dehnungen im meistbeanspruchten Querschnitt in Wandmitte (gestrichelte Kurve, $\varepsilon_{r z}=0$ ) flachen sie infolge der fehlenden Zugfestigkeit ab. Die linear verlaufenden, fein ausgezogenen Kurven beschreiben den Zusammenhang bei einem Material mit Zugfestigkeit (vgl. Bild 6.9). Nach dem Ueberschreiten eines maximalen $m_{A}$-Wertes nimmt $m_{A}$ mit zunehmender verdrehung $\vartheta_{A}$ ab und verschwindet beim Schnittpunkt der Kurve mit der Abszisse. Bei weiterer Verdrehung wird $\mathrm{m}_{A}$ negativ, d.h. die Wand stützt sich zur Erhaltung einer Gleichgewichtslage auf der darüber- und darunterliegenden Decke ab. Die Verdrehung kann nur bis zur kritischen Verdrehung $\vartheta_{A, k r}$ gesteigert werden. Bei Verdrehungen, die diesen Wert übersteigen, ist keine Gleichgewichtslage mehr möglich. Allerdings wird in den meisten Fällen $\sigma_{b}$, und unter der Annahme, dass $\sigma_{b}$ der Mauerwerksfestigkeit $\sigma_{u}$ entspricht, der Bruch bereits bei kleineren Verdrehungen erreicht. Entlang der strichpunktierten Kurve ist $\sigma_{r}=\sigma_{b}=\sigma_{u}$.

Eine Ausnahme bildet die Kurve für $\sigma_{m} / \sigma_{b}=0.001$. Die Wandenden können bis zur kritischen Wandendverdrehung verdreht werden, ohne dass $\sigma_{b} b z w$. $\sigma_{u}$ erreicht wird. Mit einem derartigen Stabilitätsversagen ist bei sehr schlanken Wänden oder geringer Axiallast $P$ bzw. $\sigma_{m} / \sigma_{b} z u$ rechnen.

In Bild 6.19 ist für Schlenkheiten von 25 und 50 der Zusammenhang zwischen der mittleren Spannung und der Verdrehung $\vartheta_{A} / \sqrt{\varepsilon_{b}}$, bei der entweder Material$\left(\sigma_{r}=\sigma_{b}=\sigma_{u}\right)$ oder Stabilitätsversagen auftritt, dargestellt. Diese Spannung

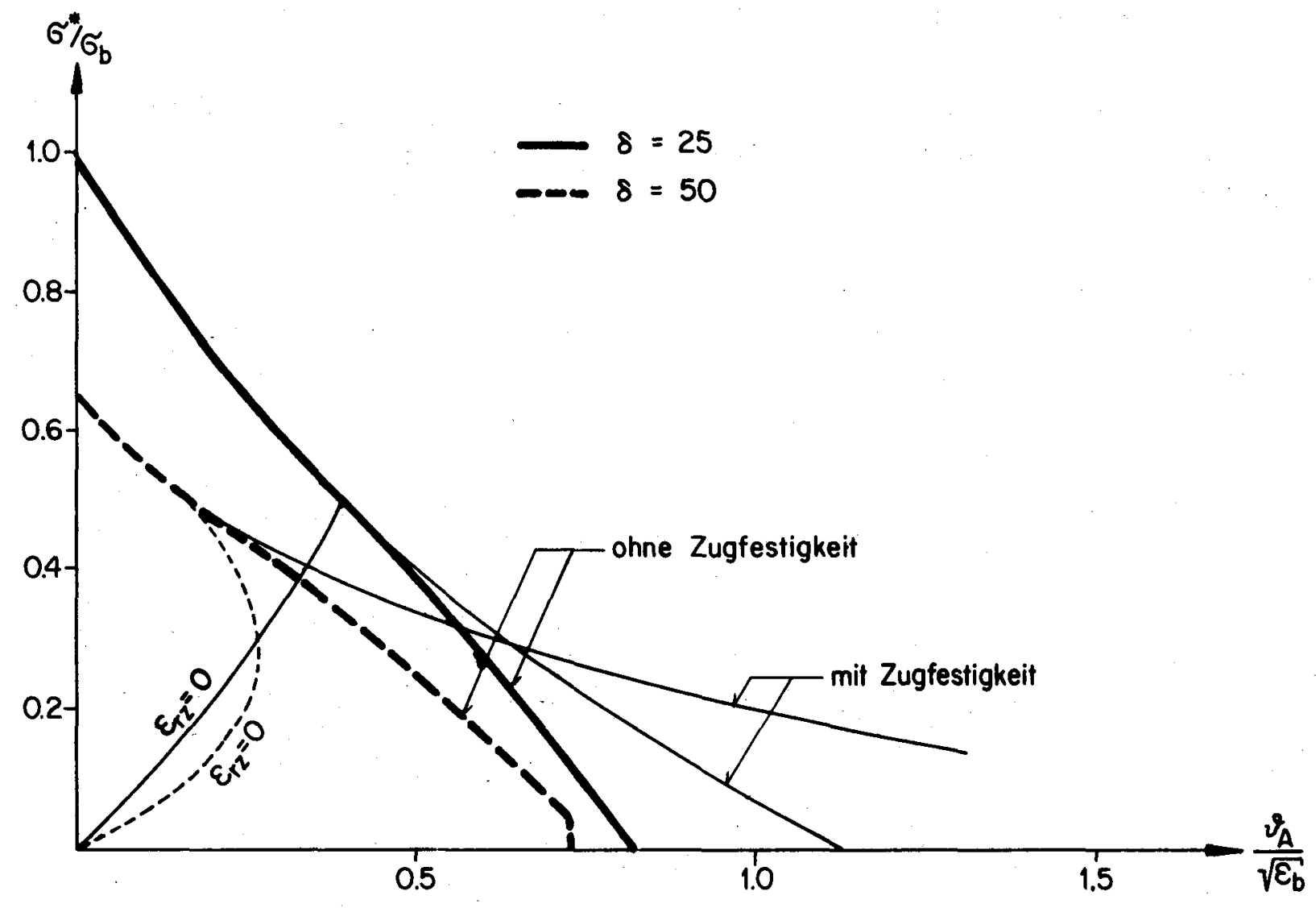

Bild 6.19: $\sigma^{*}-\nu_{A}\left(\sigma_{r}=\sigma_{b}=\sigma_{u} ; \varepsilon_{r z}=0\right)$ 
wird als Traglastspannung $\sigma^{*}$ bezeichnet. Die Bruchverdrehung nimmt mit zunehmendem $\sigma^{*}$ ab. Die Bruchkurve für die Schlankheit 50 hat bei $\sigma^{*} / \sigma_{b}=0.05$ einen markanten Knick. Im Lastbereich $0 \leq \sigma^{*} / \sigma_{b} \leq 0.05$ tritt Stabilitätsversagen auf. Die Bruchkurven für zugfestes Material verlaufen bis zum Auftreten von Dehnungen gleich wie für Material ohne Zugfestigkeit. Bei kleineren Lasten ergeben sich dann jedoch wesentlich grössere Bruchverdrehungen (vgl. Bild 6.11).

\section{Folgerungen}

Die dargestellten Lösungen der Differentialgleichungen (6.5) und (6.16) weisen teilweise grosse Unterschiede auf:

\section{- Beanspruchungsart E:}

Im Gegensatz zum linear elastischen Material mit Zugfestigkeit kann sich bei einem Material ohne Zugfestigkeit auch bei exzentrischer Belastung der Wand ein Stabilitätsversagen einstellen (Bild 6.16).

- Beanspruchungsart V:

Beim linear elastischen Material mit Zugfestigkeit ergibt sich unter konstanter Last $\left(\sigma_{m}=\right.$ konst.) ein linearer Zusammenhang zwischen dem Exzentrizitätsmass $m_{A}$ und der Wandverdrehung $\vartheta_{A}$. Demgegenüber weisen die $m_{A}{ }^{-\vartheta_{A}}-$ Kurven bei einem Material ohne Zugfestigkeit horizontale und vertikale Tangenten auf. In den Berührungspunkten mit den vertikalen Tangenten tritt eine besondere Form eines Stabilitätsversagens auf: Die Wandendverdrehung erreicht einen kritischen Wert $\vartheta_{A, k r}$ (Bild 6.18).

Die Traglastspannung $\sigma^{*}$ ist von der Wandendverdrehung $\vartheta_{A}$ abhängig. Je kleiner die Wandendverdrehung $\vartheta_{A}$, desto grösser ist die Traglastspannung $\sigma^{*}$ (Bild 6.19).

Aus dem Verlauf der experimentell bestimmten e- $\Phi-K u r v e n$ (vgl. Bilder 5.4, 5.6, 5.7) war ersichtlich, dass die Zugfestigkeit des untersuchten Backsteinmauerwerks sehr gering war. Es darf daher angenommen werden, dass die dargestellte Lösung auf der Grundlage des linear elastischen Materials ohne Zugfestigkeit das Tragverhalten von Backsteinwänden qualitativ richtig zu beschreiben vermag. 


\subsection{Numerische Verfahren}

\subsubsection{Ueberblick}

Für die Herleitung der Differentialgleichung (5.16) wurden zwei Annahmen getroffen, die bei Backsteinmauerwerk sowie bei Mauerwerk im allgemeinen nicht unbedingt zutreffend sind:

- Material mit linear elastischem Stoffgesetz ohne Zugfestigkeit

- Vollquerschnitt

Die in Bild 3.4 dargestellten experimentell bestimmten Spannungs-StauchungsKurven und das Stoffgesetz von Bild 5.5 weisen eine nichtlineare Beziehung zwischen Spannung und Stauchung auf, so dass mit dem linear elastischen Stoffgesetz ohne Zugfestigkeit das tatsächliche Materialverhalten nur angenähert beschrieben wird. Die Annahme eines Vollquerschnittes entspricht nicht der Wirklichkeit. Die Mauersteine, insbesondere Backsteine und Kalksandsteine sind üblicherweise gelocht. In vielen Fällen beträgt der Lochflächenanteil bis zu 50\%. In Abschnitt 4.5 wurde gezeigt, dass die Lochung einen grossen Einfluss auf das e- $\Phi$-Verhalten sowie auf die Interaktion M-P hat und demzufolge nicht vernachlässigbar ist.

Nichtlineares Materialverhalten und beliebige Lochungen der Mauersteine können nur mit numerischen Verfahren berücksichtigt werden. Im Rahmen dieser Arbeit wurde ein entsprechendes Computerprogramm für Mauerwerkswände unter Axiallast und Biegung entwickelt. Die numerische Berechnung des Tragverhaltens erfolgt an einem Starkörper-Feder-Modell und basiert auf den Column Deflection Curves (vgl. Abschnitte 6.3.3 und 6.4). Als Eingabedaten benötigt das Computerprogramm e- $\Phi-K u r v e n$. Sie wurden in den Kapiteln 4 und 5 eingehend erläutert.

Im Folgenden soll das Tragverhalten von Maverwerkswänden mit einem einfachen Federmodell beschrieben und der Vebergang zum verfeinerten Federmodell, dem Starrkörper-Feder-Modell gemacht werden.

\subsubsection{Einfaches Federmiodell}

Die symmetrisch belastete Wand von Bild 6.20 a sei durch das einfache Federmodell in Bild 6.20 b charakterisiert. Die gesamte Verdrehung der stetig gekrümmten Wand ergibt sich durch die Integration der Krümmungen längs der Wandaxe. Sie beträgt $2 \cdot \vartheta_{A}$. Beim Federmodell ist diese durch die Summe der Rotationen der Federn $F_{A}, F_{B}$ und $F_{C}$ bestimmt.

Um einen quantitativen Vergleich des Tragverhaltens mit der analytischen Lösung von Angervo anstellen zu können, wird bei der Bestimmung der Federcharakteristiken von $F_{A}, F_{B}$ und $F_{C}$ wiederum von einem linear elastischen $M a-$ terial ohne Zugfestigkeit und einem Vollquerschnitt ausgegangen. Selbstver- 
ständlich könnten der Berechnung der Federcharakteristiken beliebige Stoffgesetze und Lochungen zugrunde gelegt werden.

Unter Benützung der Gleichungen (4.6) und (4.15) ergeben sich dann die untenstehenden Beziehungen. Die Federdrehwinkel $\varphi_{A}$ und $\varphi_{C}$ entsprechen den über einen Viertel bzw. eine Hälfte der Wandhöhe multiplizierten Krümmungen. Die Federcharakteristiken von $F_{A}$ und $F_{C}$ sind voraussetzungsgemäss gleich.

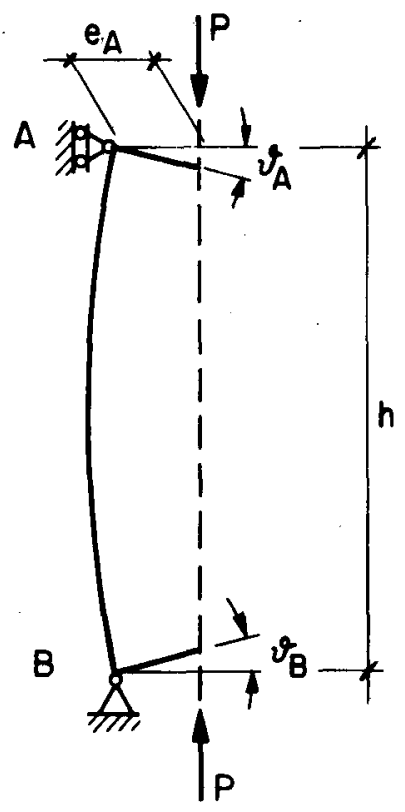

a)
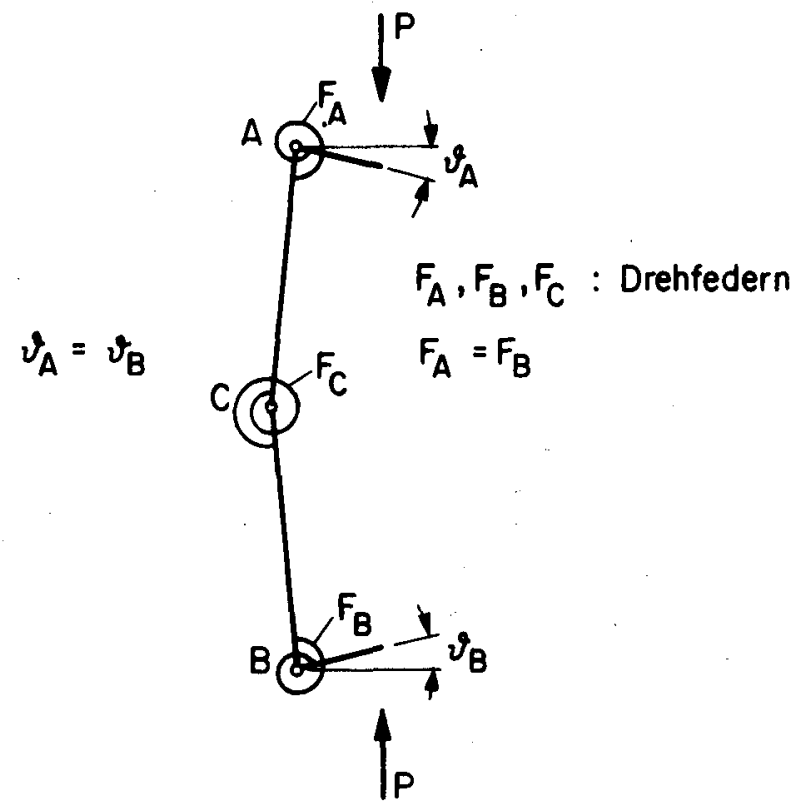

b)

\section{Bild 6.20: Symmetrisch belostete Wand-Einfaches Federmodell}

Federcharakteristiken:

$$
\left.\begin{array}{rl}
F_{A}=F_{B}: & \frac{e_{A}}{d} \leq \frac{1}{6}: \varphi_{A}=\varphi_{B}=\Phi_{A} \cdot \frac{h}{4}=3 \cdot \delta \cdot \frac{P}{P_{b}} \cdot \varepsilon_{b} \cdot \frac{e_{A}}{d} \\
\frac{1}{6} \leq & \frac{{ }^{e} A}{d} \leq \frac{1}{2}: \varphi_{A}=\varphi_{B}=\Phi_{A} \cdot \frac{h}{4}=\frac{2}{9} \cdot \delta \cdot \frac{P}{P_{b}} \cdot \varepsilon_{b} \cdot \frac{1}{\left(1-2 \cdot \frac{e_{A}}{d}\right)^{2}} \\
F_{C}: \quad \frac{{ }^{C} C}{d} \leq \frac{1}{6}: \varphi_{C}=\Phi_{C} \cdot \frac{h}{2}=6 \cdot \delta \cdot \frac{P}{P_{b}} \cdot \varepsilon_{b} \cdot \frac{e_{C}}{d} \\
\frac{1}{6} \leq \frac{{ }^{e} C}{d} \leq \frac{1}{2}: \varphi_{C}=\Phi_{C} \cdot \frac{h}{2}=\frac{4}{9} \cdot \delta \cdot \frac{P}{P_{b}} \cdot \varepsilon_{b} \cdot \frac{1}{\left(1-2 \frac{{ }_{C} C}{d}\right)^{2}}
\end{array}\right\}
$$

Die Grössen $P_{b}$ und $\varepsilon_{b}$ entsprechen den Bezugswerten von Kapitel 4, Gl. (4.1). Die Gleichgewichtsbedingung (6.3) ist in den Punkten $A, B$ und $C$ zu erfüllen, womit sich die Wandendverdrehung $\vartheta_{A}$ wie folgt angeben lässt: 


$$
\vartheta_{A}=\varphi_{A}+\frac{2}{\delta} \cdot\left(\frac{{ }^{e} C}{d}-\frac{{ }^{e} A}{d}\right)
$$

und die kinematische Bedingung lautet

$$
\varphi_{C}=\frac{4}{\delta} \cdot\left(\frac{e_{C}}{d}-\frac{e_{A}}{d}\right)
$$

Nach Einsetzen der Federcharakteristiken (Gleichungen (6.28 und (6.29)) in die Gleichungen (6.30) und (6.31) lauten die Beziehungen zur Beschreibung des Tragverhaltens wie folgt:

$\left.\begin{array}{l}0 \leq \frac{e_{A}}{d} \leq \frac{1}{6} \\ 0 \leq \frac{e C}{d} \leq \frac{1}{6}\end{array}\right\}: \quad \frac{\vartheta_{A}}{\sqrt{\varepsilon_{b}}}=6 \cdot \frac{P}{P_{b}} \cdot \frac{e_{A}}{d} \cdot \sqrt{\varepsilon_{b}} \cdot \delta \cdot \frac{\left(4-3 \cdot \frac{P}{P_{b}} \cdot \delta^{2} \cdot \varepsilon_{b}\right)}{\left(4-6 \cdot \frac{P}{P_{b}} \cdot \delta^{2} \cdot \varepsilon_{b}\right)}$

$\left.\begin{array}{l}0 \leq \frac{e_{A}}{d} \leq \frac{1}{6} \\ \frac{1}{6} \leq \frac{e_{C}}{d} \leq \frac{1}{2}\end{array}\right\}: \quad \frac{\vartheta_{A}}{\sqrt{\varepsilon_{b}}}=\frac{P}{P_{b}} \cdot \delta \cdot \sqrt{\varepsilon_{b}} \cdot\left\{3 \cdot \frac{e_{A}}{d}+\frac{2}{9} \cdot \frac{1}{\left(1-2 \cdot \frac{e_{C}}{d}\right)^{2}}\right\}$

$\left.\begin{array}{l}\frac{1}{\sigma} \leq \frac{e_{A}}{d} \leq \frac{1}{2} \\ \frac{1}{\sigma} \leq \frac{e_{C}}{d} \leq \frac{1}{2}\end{array}\right\}: \quad \frac{\vartheta_{A}}{\sqrt{\varepsilon_{b}}}=\frac{2}{9} \cdot \frac{P}{P_{b}} \cdot \delta \cdot \sqrt{\varepsilon_{b}} \cdot\left\{\frac{1}{\left(1-2 \cdot \frac{{ }^{B} A}{d}\right)^{2}}+\frac{1}{\left(1-2 \cdot \frac{{ }^{e} C}{d}\right)^{2}}\right\}$

In Bild 6.21 ist der. Zusammenhang zwischen dem Exzentrizitätsmass $m_{A}$ und der Wandendverdrehung $\vartheta_{A}$ für konstante Spannungsverhältnisse $\sigma_{m} / \sigma_{b}=0.1,0.3$ und 0.5 dargestelit. Nach einem linearen Anstieg flachen die Kurven ab, überschreiten ein Maximum und schneiden die Abszisse bei zunehmender Verdrehung. Der kritische Wandendverdrehungswinkel ist deutlich erkennbar. Aus dem Vergleich mit den gestrichelten Kurven (vgl, auch Bild 6.18) ist ersichtlich, dass mit dem Federmodell das Tragverhalten qualitativ richtig erfasst werden kann. Quantitativ ergeben sich jedoch zur analytischen Lösung von Angervo vor allem im Bereich grösserer Verdrehungen $\vartheta_{A}$ und Spannungen $\sigma_{m} / \sigma_{b}$ deutliche Abweichungen.

Mit einer feineren Unterteilung der Wand in Federn und starre Verbindung kann das Modell verbessert werden (Bild 6.22). 


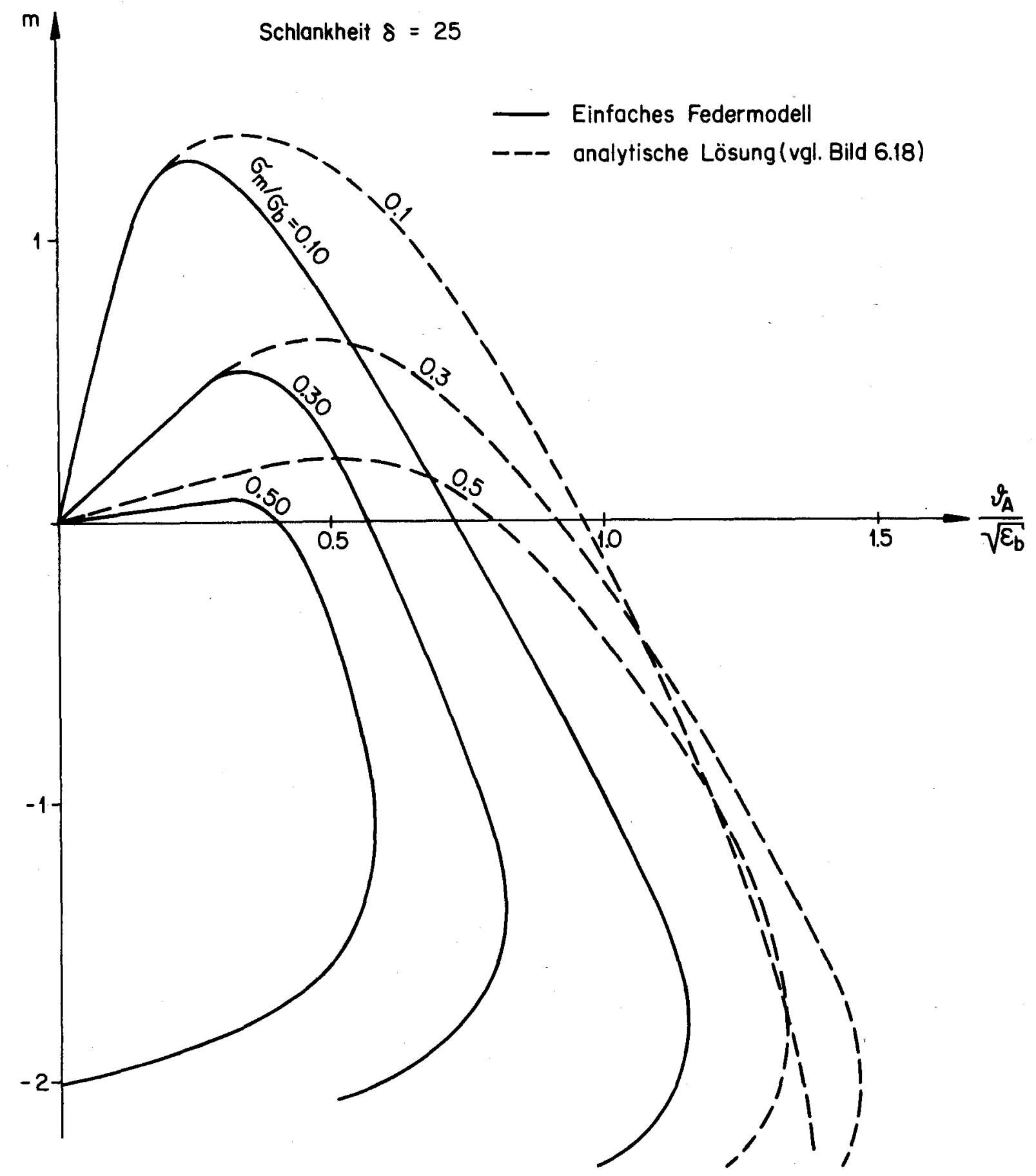

Bild 6.21: $m_{A}-v_{A}\left(\sigma_{m} / \sigma_{b}=\right.$ konst.) (Vergleich: Federmodell -analytische Lösung) 


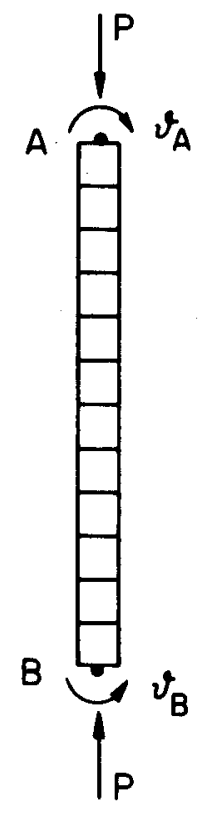

o)

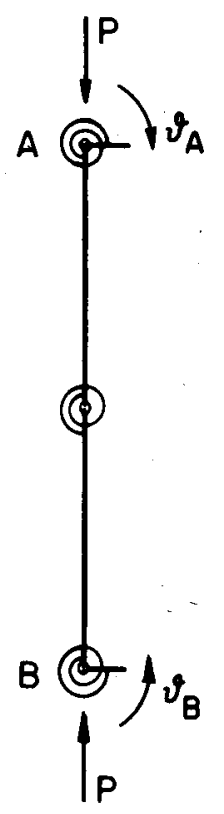

b)

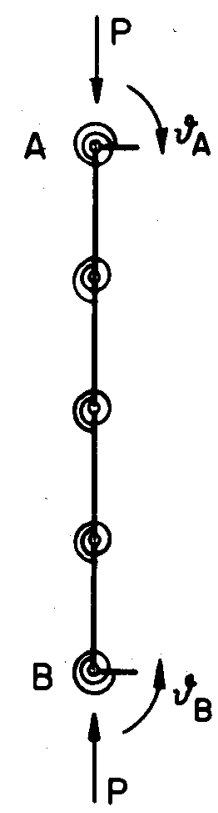

c)

\section{Bild 6.22: Mauerwerkwand - Modelle}

Derartige, verfeinerte Modelle, sie werden in den folgenden Untersuchungen als Starrkörper-Feder-Modelle bezeichnet, können im Gegensatz zum einfachen Federmodell nur numerisch behandelt werden. In Bild 6.23 ist der Einfluss der Länge $\Delta x$ der Starrkörper auf die $m_{A}{ }^{-\vartheta}{ }_{A}$-Beziehung der symmetrisch belasteten Wand von Bild 6.22 a für Spannungsverhältnisse $\sigma_{m} / \sigma_{b}=0.1$ und 0.3 dargestel1t. Die $m_{A}{ }^{-\vartheta}{ }_{A}$-Kurven des Starrkörper-Feder-Modells wurden mit dem eingangs erwähnten Computerprogramm berechnet. Für die Bestimmung der Federcharakteristiken wurde wiederum von einem linear elastischen Stoffgesetz ohne Zugfestigkeit und einem Vollquerschnitt ausgegangen. Die stark ausgezogenen Kurven entsprechen der analytischen Lösung von Angervo (vgl. gestrichelte Kurve in Bild 6.21). In den Endpunkten der Kurven ist die Randspannung $\sigma_{r}$ im meistbeanspruchten Querschnitt in Wandmitte gleich $\sigma_{b}$. 

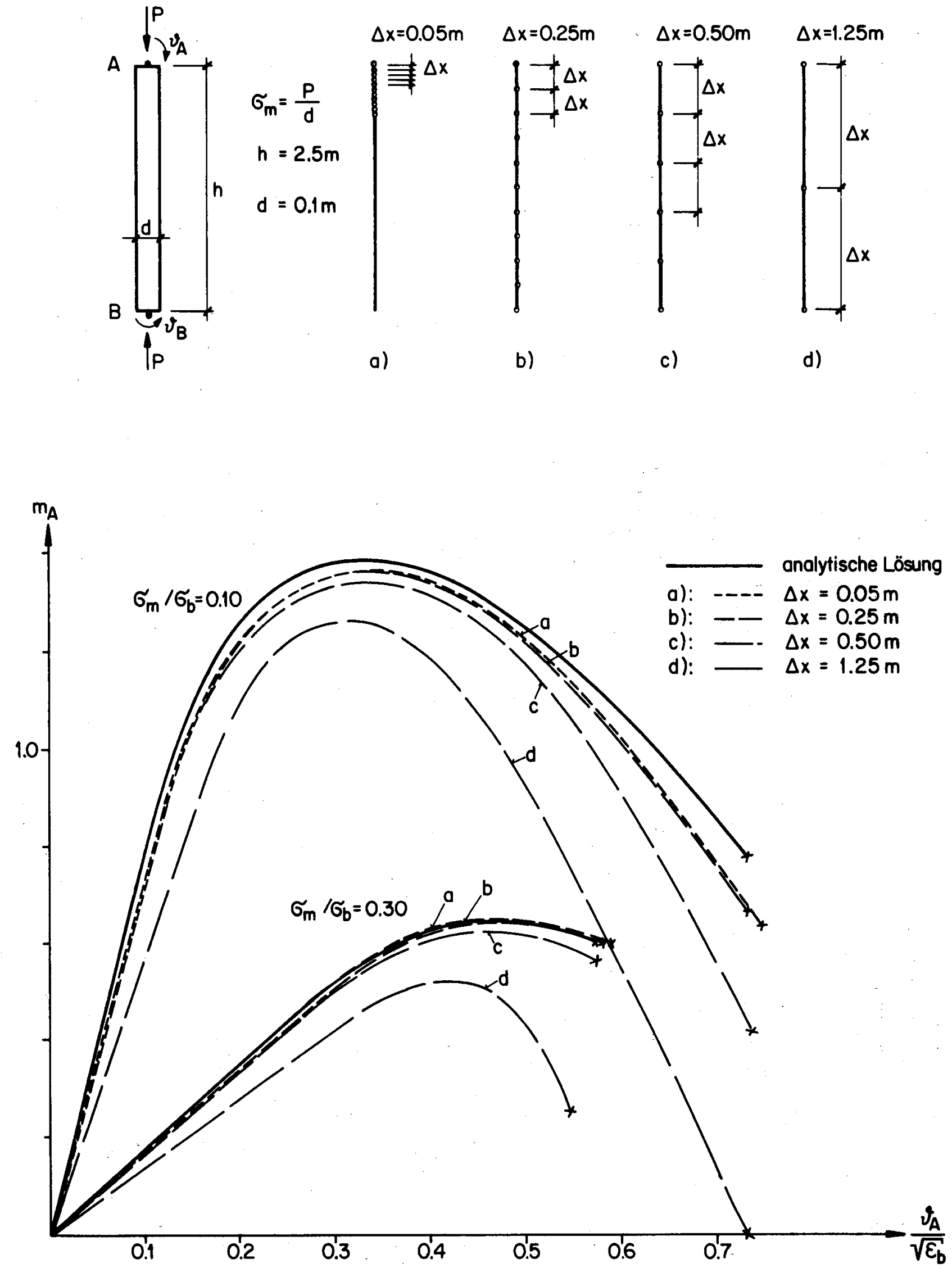

Bild 6.23: $m_{A}-\nu_{A}\left(\sigma_{m} / \sigma_{b}=\right.$ konst. $):$ Vergleich analytische Lösung-Starrkörper-Federmodell 
Die Länge $\Delta x$ der Starrkärper ist für den Verlauf der $m_{A}{ }^{-\vartheta}{ }_{A}^{-K u r v e n ~ v o n ~ g r o s-~}$ sem Einfluss. Für $\Delta x$-Werte von 0.05 und $0.25 \cdot m$ erhält man näherungsweise die gleichen Resultate wie mit der Lösung von Angervo. Für grössere $\Delta x$ sind die Abweichungen grösser.

\section{3 .3 Column Deflection Curves (CDC)}

Der Begriff Column Deflection Curve geht auf eine Arbeit von von Karman 1910 [18] zurück. Chwalla [4] verallgemeinerte die Anwendung der CDC auf verschiedene Querschnitte und statisch unbestimmte Probleme im Jahre 1937 [23].

Die Wand in Bild 6.24 ist durch die Axiallast P exzentrisch belastet. Bei linear elastischem Materialverhalten kann die Momentengleichgewichtsbedingung (6.3) in eine geschlossen lösbare Differentialgleichung (6.5) übergeführt werden (Abschn. 6.2.1). Die Lage der verformten Wandaxe bezüglich der Wirkungslinie von $P$ ist durch die Beziehung zwischen der Exzentrizität e und der $x$-Koordinate bestimmt (GI. (6.11)).

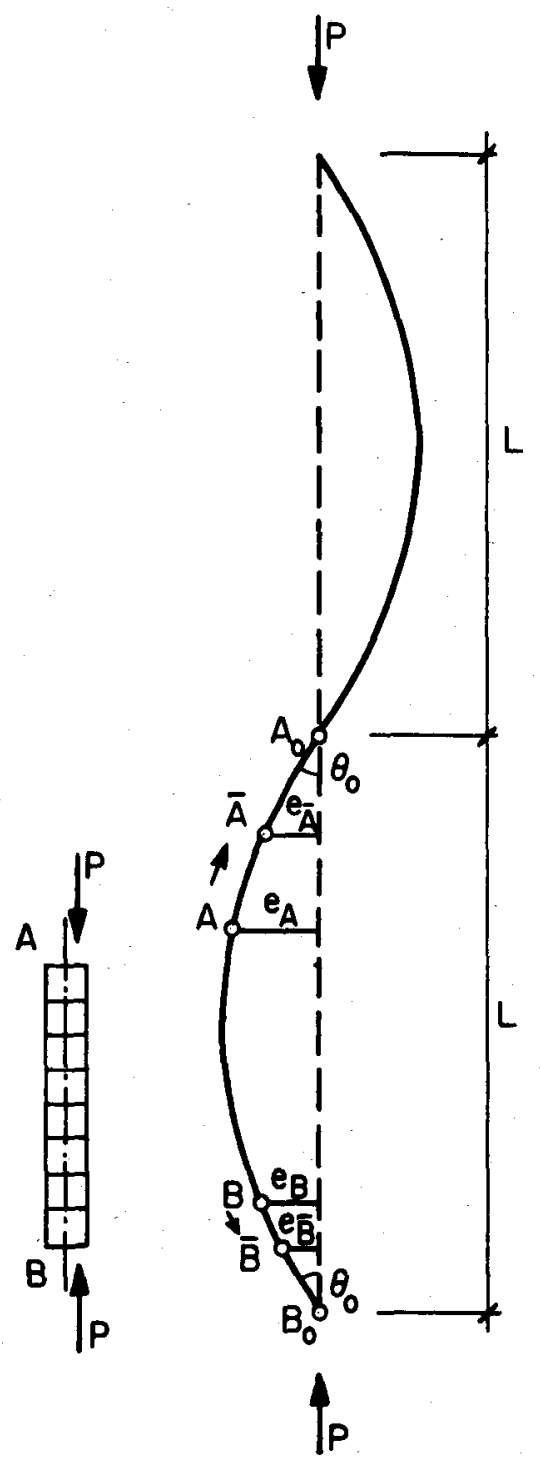

Bild 6.24: Column Deflection Curve (CDC) 
In den Punkten $A$ und $B$ beträgt die Exzentrizität $e_{A}$ und $e_{B}$. In jedem Schnitt der Wandaxe ist die Gleichgewichtsbedingung erfüllt. Wird die betrachtete Wand nun über die Punkte $A$ und $B$ hinaus verlängert, so ergibt sich für den Wandabschnitt die gleiche verformte Lage der Wandaxe, wenn die Exzentrizitäten in den Punkten $\bar{A}$ und $\bar{B} e_{\bar{A}}$ und $e_{\bar{B}}$ betragen. Im Grenzfall kommt $A$ in $A_{0}$ und $B$ in $B_{0}$ zu liegen. Der Bogen $A_{0} B_{0}$ entspricht dann der einen Hälfte einer CDC. Die ganze CDC entspricht einer Doppelwelle der Länge 2.L (Bild 6.24). Der Schnittwinkel, d.h. der Winkel zwischen der Wandaxe und der Wirkungslinie von $P$ beträgt in den beiden Endpunkten $\theta_{0}$. Die Kurve ist also symmetrisch. In jedem Punkt ist die Gleichgewichtsbedingung erfüllt. Der Wandabschnitt $A B$ werde nun entlang der CDC verschaben. Die neue Lage ist durch die Endpunkte $\bar{A}$ und $\bar{B}$ gekennzeichnet (Bild 6.25 ).

Die Exzentrizität in diesen beiden Punkten hat sich dementsprechend verändert. Der dazwischenliegende Abschnitt der CDC beschreibt die neue verformte Lage der Wandaxe. Die sich bei den Exzentrizitäten $e_{A}$ und $e_{B}$, der Axiallast $P$ sowie der Wandhöhe $h$ ergebenden Wandendverdrehungen $\vartheta_{A}$ und $\vartheta_{B}$ könrien folgendermassen bestimmt werden:

$$
\begin{aligned}
& \vartheta_{A}=\vartheta_{A}-\gamma_{A} \\
& \vartheta_{B}=\theta_{B}+\gamma_{B}
\end{aligned}
$$
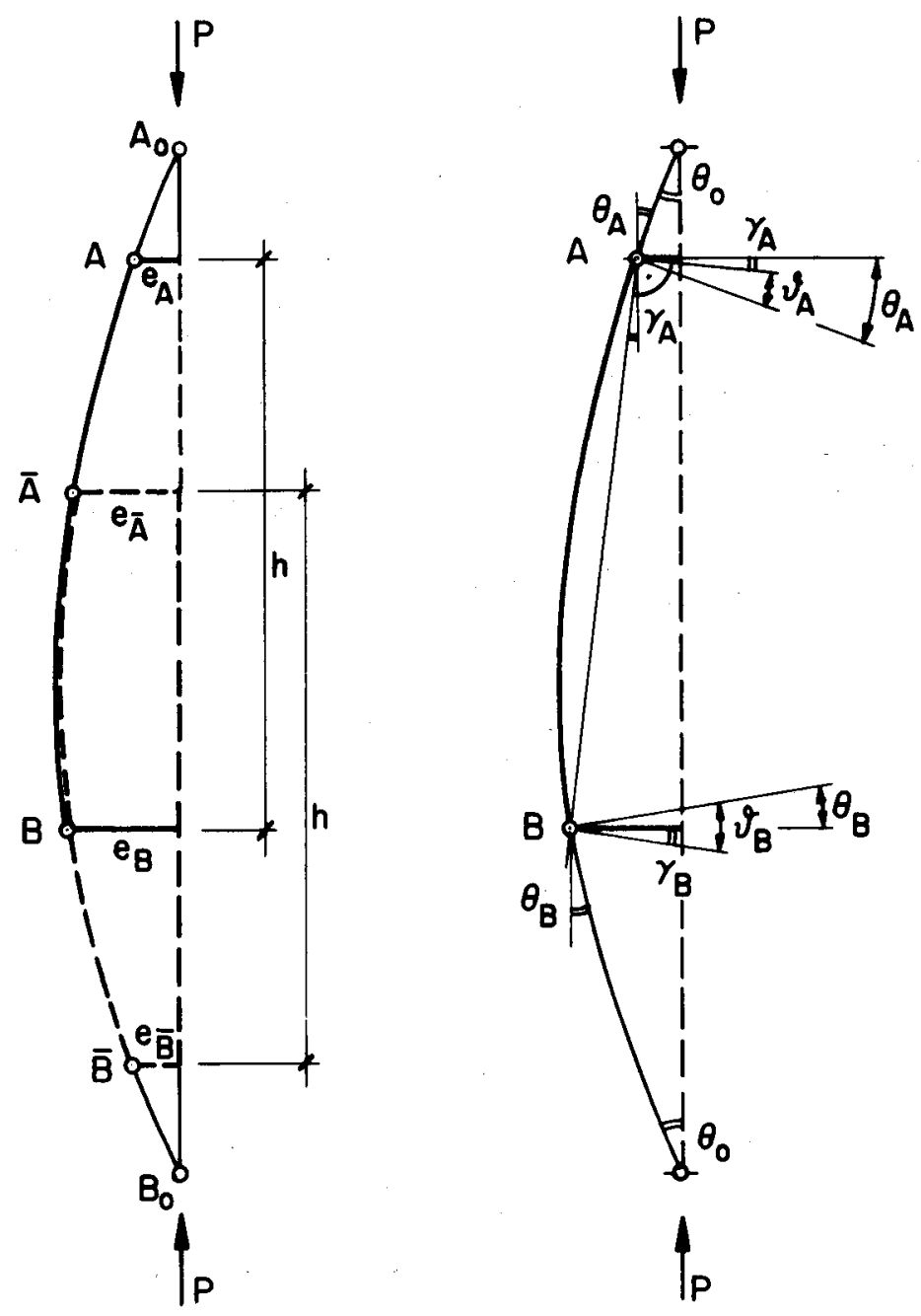

$$
\begin{aligned}
& v_{A} \theta_{A}-r_{A} \\
& r_{A}=r_{B}=\frac{e_{B}-e_{A}}{h}
\end{aligned}
$$$$
\vartheta_{B}=\theta_{B}+\gamma_{B}
$$

Bild 6.25: Wandabschnitt $A B$ auf $C D C$ 
Beim linear elastischen Stoffgesetz hat die CDC die Form einer Sinuswelle als Lösung der Gl. (6.5). Die Sinus-Halbwelle in Bild 6.24 entspricht dann der ausgeknickten Lage einer zentrisch belasteten Wand unter $P_{k r}$. Die Länge $L$ beträgt demnach $\pi \cdot \sqrt{E I / P_{k r}}$ und ist unabhängig vom Winkel $\theta_{0}$ bzw. von der Exzentrizität in Kurvenmitte. Für eine Last $P$ existieren also beliebig viele Column Deflection Curves gleicher Länge. In Bild 6.26 ist eine Schar von Column Deflection Curves dargestellt. Sie gelten für die konstante Axiallast P.

Bei nichtlinearem Materialverhalten wird die Länge L durch überproportional zur Exzentrizität wachsende Krümmungen vermindert $\left(L_{\rightarrow} L^{\prime} \rightarrow L^{\prime \prime}\right)$. Für eine Last $P$ existieren dann beliebig viele CDC's unterschiedlicher Länge.

Column Deflection Curves sind verformte Axen von axial belasteten Wänden, die mit den äusseren Lasten in allen oder diskreten Punkten im Gleichgewicht sind.

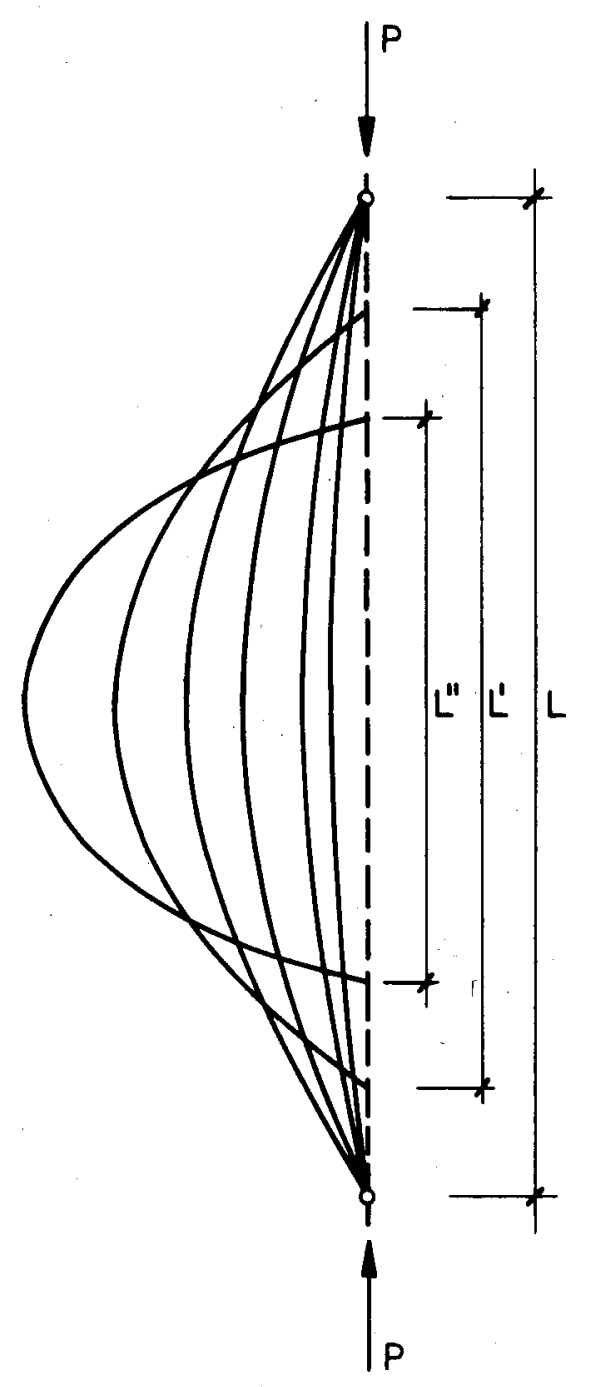

Bild 6.26: $\mathrm{CDC}-$ Schar 


\section{Die Berechnung der Column Deflection Curves}

Die Berechnung der CDC kann beim nichtlinearen Materialverhalten in der Regel nur numerisch durchgeführt werden. Für einige spezielle nichtlineare Stoffgesetze und idealisierte Querschnitte sind geschlossene Lösungen bekannt [23] . Bei geschlossenen Lösungen sind die Gleichgewichtsbedingungen in allen Punkten der CDC erfüllt. Demgegenüber vermögen numerische Berechnungen diese nur in diskreten Punkten der CDC zu erfüllen.

Mit einem numerischen Integrationsverfahren erhält man in diskreten Schritten $i$ die Exzentrizität $e_{i}$ und den Kurvenwinkel $\theta_{i}$. Mit $\theta_{i}$ wird die Neigung der COC gegenüber der Wirkungslinie von $P$ im Schnitt $i$ bezeichnet. Bei bekanntem Stoffgesetz und Wandquerschnitt können in Abhängigkeit der Axiallast $P$ Exzentrizitäts-Krümmungs-Beziehungen ermittelt werden. Deren Berechnung kann analytisch (Kap. 4) oder numerisch (Kap. 5) erfolgen.

Als Ausgangspunkt für die Berechnung der CDC wählt man zweckmässigerweise entweder den mittleren Schnitt der CDC und trifft eine Annahme über die Exzentrizität $e_{0}$ oder man geht von einem Ende der CDC aus und gibt den Anfangswinkel $\theta_{0}$ vor (Bild 6.27).

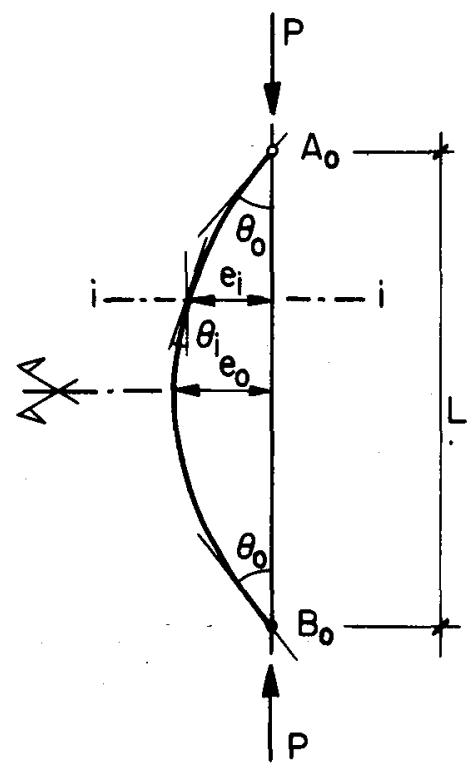

Bild 6.27: Ausgangspunkte für die Berechnung der CDC

Der Integrationsprozess wird mit Hilfe von Bild 6.28 erläutert. Ausgangspunkt sei der Querschnitt in der Mitte der CDC. Die Axiallast betrage $P$ und die Exzentrizität $e_{0}$ ist in diesem Schnitt gleich null. Im Integrationsintervall $\Delta x$ sei die Krümmung konstant. Die entlang der CDC aufgetragene Krümmung weist demnach einen treppenförmigen Verlauf auf. 
Die Krümmung $\Phi_{0}$ im Ausgangsquerschnitt ergibt sich aus der ExzentrizitätsKrümmungs-Beziehung. Unter der Annahme konstanter Krümmungen im Abschnitt $\Delta x$ beträgt die Exzentrizität im Schnitt 1 :

$$
e_{1}=e_{0}-\Phi_{0}\left(e_{0}\right) \cdot \frac{\Delta x^{2}}{2}
$$

und die Neigung $\theta_{1}$ der CDC gegenüber der Wirkungslinie von P:

$$
\theta_{1}=\theta_{0}+\Phi_{0}\left(e_{0}\right) \cdot \Delta x
$$

Im Schnitt 2 lauten dann diese Beziehungen:

$$
\begin{aligned}
& e_{2}=e_{1}-\Phi_{1}\left(e_{1}\right) \cdot \frac{\Delta x^{2}}{2}-\theta_{1} \cdot \Delta x \\
& \theta_{2}=\theta_{1}+\Phi_{1}\left(e_{1}\right) \cdot \Delta x
\end{aligned}
$$

Dieses Verfahren kann nun entsprechend fortgeführt werden. Allgemeine ergeben sich folgende Beziehungen:

$$
\begin{aligned}
& e_{i+1}=e_{i}-\Phi_{i}\left(e_{i}\right) \cdot \frac{\Delta x^{2}}{2}-\theta_{i} \cdot \Delta x \\
& \theta_{i+1}=\theta_{i}+\Phi_{i}\left(e_{i}\right) \cdot \Delta x
\end{aligned}
$$

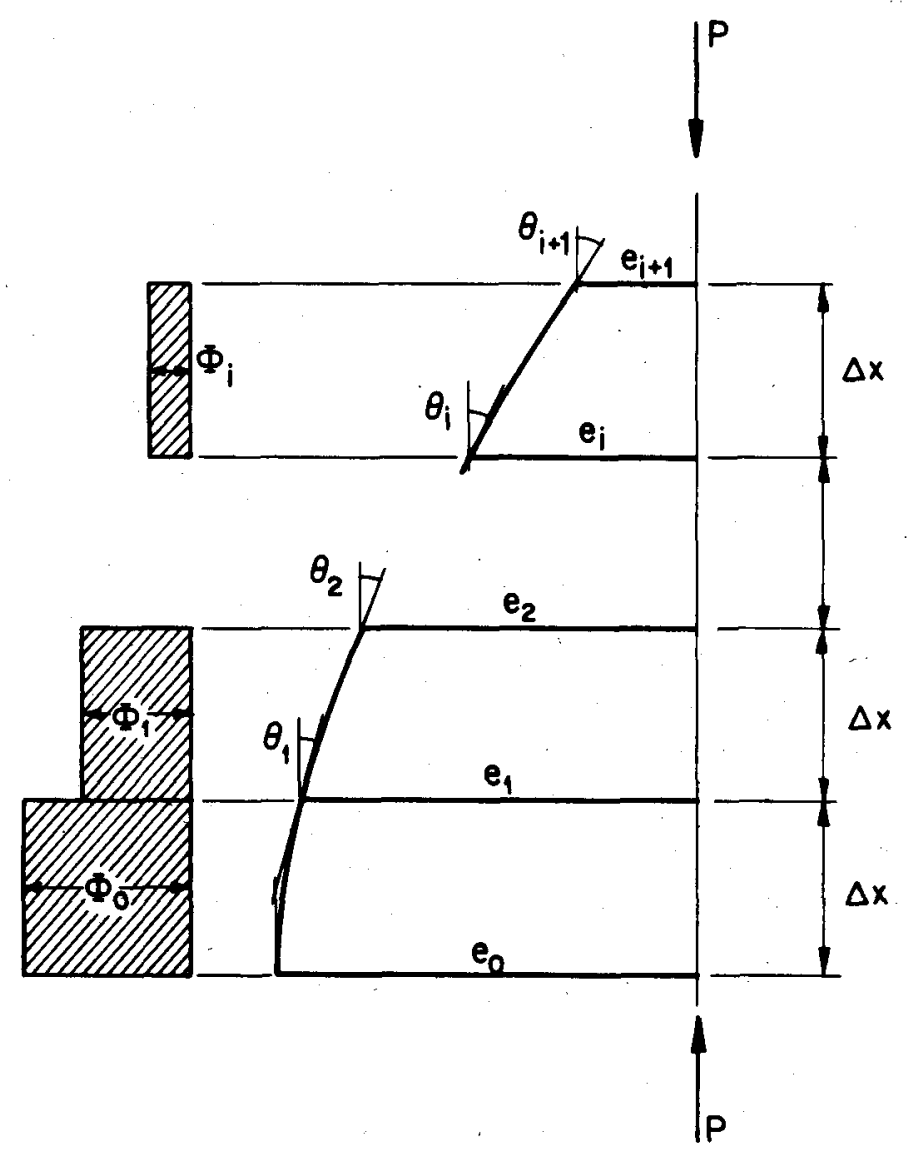

Bild 6.28: Berechnungsvorgang der CDC 
Bei verschwindender Exzentrizität entspricht der berechnete CDC-Abschnitt genau einem Viertel der CDC. Aus Symmetriegründen ist dann die ganze CDC bekannt. Dieser Abschnitt kann durch geeignete Wahl von $\Delta x$ beliebig genau erhalten werden.

Durch Variation der Exzentrizität e。 im Ausgangsquerschnitt srhält man für die konstante Axiallast $P$ eine ganze Schar von Column Deflection Curves. Die Axiallast $P$ kann dabei als Scharparameter bezeichnet werden. In Bild 6.29 ist eine solche Schar für das Stoffgesetz in Bild 5.5 dargestellt. Die Axiallast $P$ beträgt $165 \mathrm{kN} / \mathrm{m}$. Die Reduktion der CDC-Länge im nichtlinearen Bereich ist deutlich erkennbar. Sie ergibt sich bei Vergrösserung der Exzentrizität $e_{0}$ in der Mitte. Die Länge L im elastischen Bereich des Materials beträgt $18.0 \mathrm{~m}$. Die Axiallast $P$ entspricht in diesem Fall der Eulerlast.

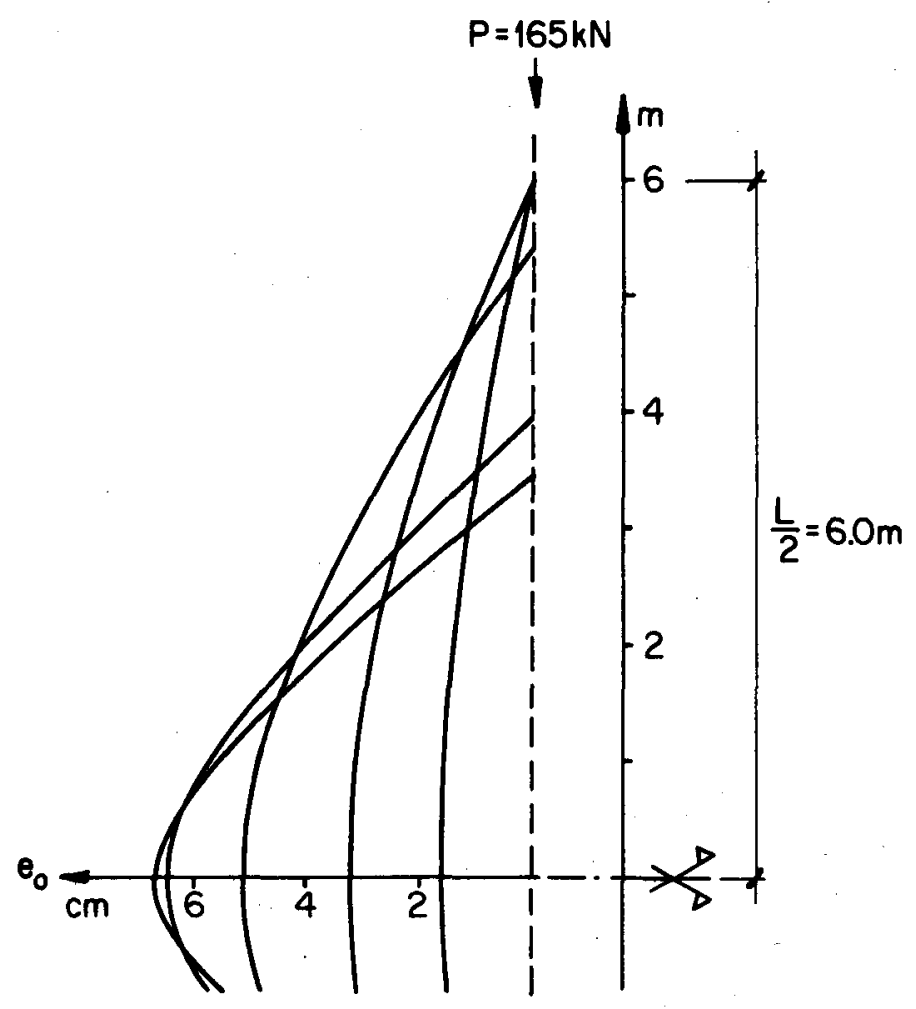

Bild 6.29: $\operatorname{CDC}$ für $P=165 \mathrm{kN} / \mathrm{m}^{\prime}$ 


\subsubsection{Anwendung der Column Deflection Curves}

Aus der Kurvenschar in Bild 6.29 kann nun für axialbelastete Wände das Tragverhalten ermittelt werden. Das Vorgehen wird im Folgenden für die Beanspruchungsarten $V$ und $E$ ( $v g l$. 6.1) beschrieben.

Beanspruchungsart $V$

A

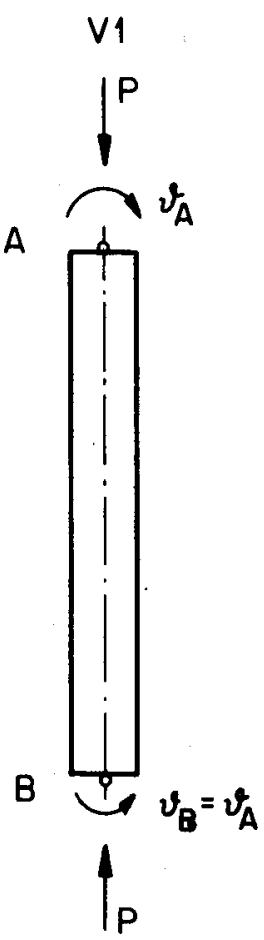

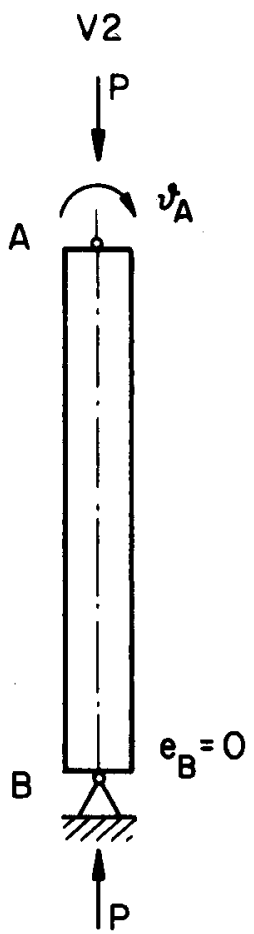

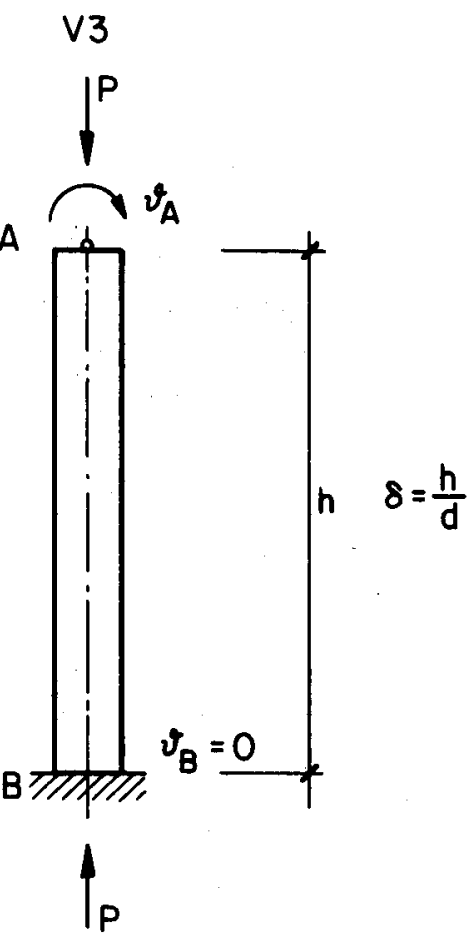

\section{Bild 6.30 : Fälle der Beanspruchungsart $V$}

Für die symmetrisch belastete Wand (Fall V1) von Bild 6.30 lassen sich aus der Schar der CDC die Exzentrizitäts-Verdrehungs-Kurven einfach bestimmen (Bild 6.31). Die Schlankheit der Wand beträgt $\delta$.

Die Wand $A B$ liegt bezüglich der Symmetrieaxe der CDC symmetrisch. Schnitte durch die Wandendpunkte $A$ und $B$ schneiden die Wirkungslinie in $A^{\prime}$ und $B^{\prime}$ und die $\operatorname{CDC}$ in $A$ und $B$. Die Strecken $A A^{\prime}$ und $B B^{\prime}$ sind gleich gross. A $A^{\prime}$ entspricht der Exzentrizität $e_{A}$. Die dazugehörige Wandendverdrehung $\vartheta_{A}$ ist gleich der Neigung $\theta_{A}$ der CDC gegenüber der Wirkungslinie von $P$. Für jede CDC lässt sich nun ein solches Wertepaar $e_{A}$, $\vartheta_{A}$ ermitteln. Trägt man die Wandendverdrehung auf der Abșzisse und die Exzentrizitäten auf der Ordinate auf, so ergibt sich für die symmetrisch belastete Wand der in Bild 6.34 schematisch dargestellte Kurvenverlauf. Die Axiallast $P$ ist entlang der $e_{A}-$ $\vartheta_{A}$-Kurve konstant. Qualitativ ähnliche Kurven wurden bereits mit der geschlossenen Lösung von Angervo (Bild 6.19) für ein linear elastisches Material ohne Zugfestigkeit erhalten.

Bei der einseitig zentrisch gelagerten Wand (Fall V2, Bild 6.30) ist die Exzentrizität an einem Wandende voraussetzungsgemäss gleich null. Die einzelnen 


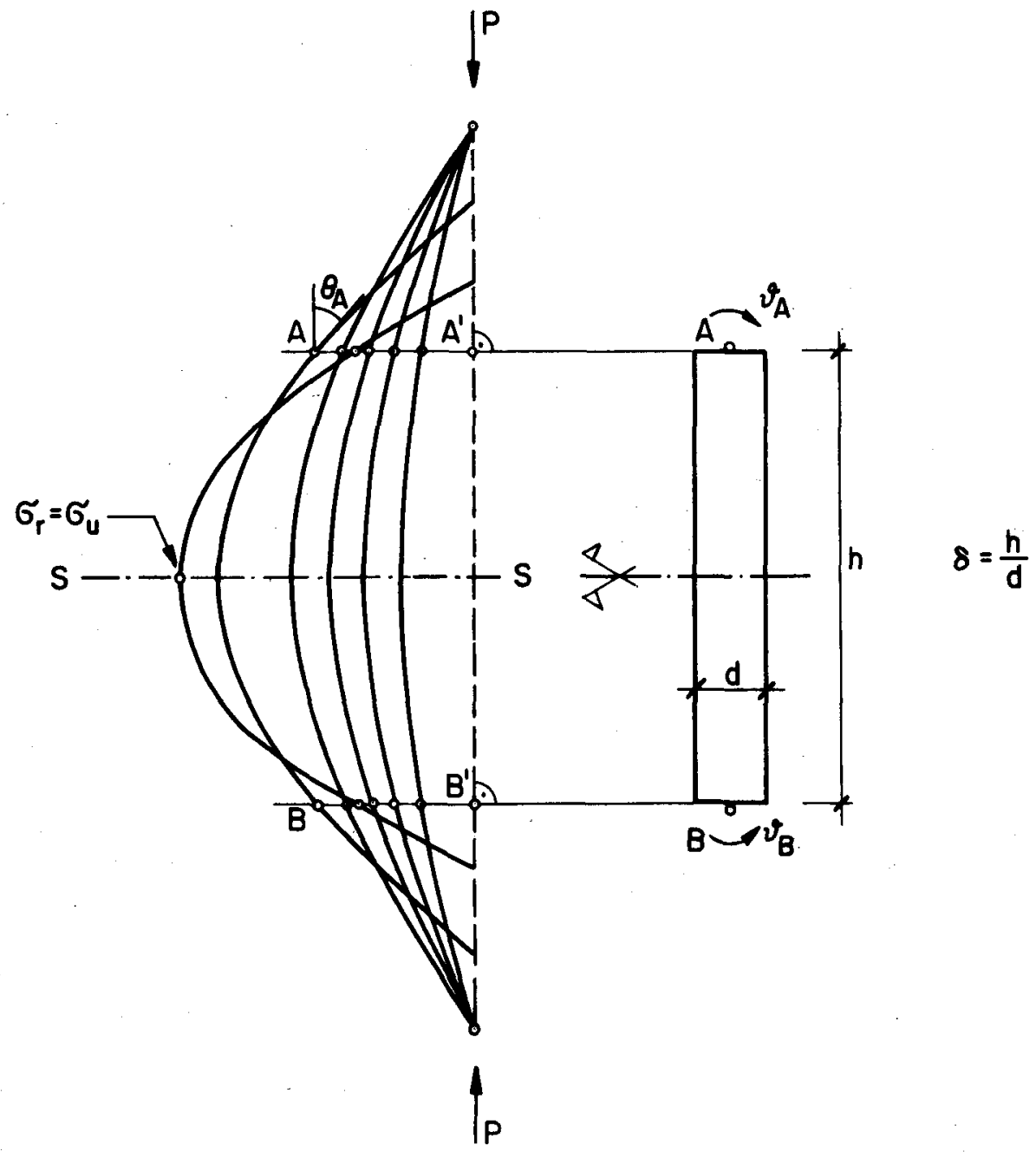

Bild 6.31: Symmetrisch belastete Wand (Fall V1)

COC der Kurvenschar sind demnach derart längs der Wirkungslinie von $P$ zu verschieben, dass die Schnittpunkte von CDC und Wirkungslinie auf der Höhe dieses Wandendes liegen. Es ergibt sich dann die in Bild 6.32 wiederum schematisch dargestellte Kurvenschar. Die ${ }_{A}{ }^{-\vartheta} A^{-K u r v e n ~ w e r d e n ~ a n a l o g ~ F a l l ~ V 1 ~ b e-~}$ stimmt und weisen grundsätzlich den gleichen Verlauf auf (Bild 6.34). Die Normale durch das andere Wandende schneidet die Wirkungslinie in $A$ ' und die $C D C$ in $A$. Die Exzentrizität ${ }_{A}$ entspricht der Strecke $A A^{\prime}$ und die Wandendverdrehung $\vartheta_{A}$ beträgt:

$$
\vartheta_{A}=\frac{e_{A}}{h}+\theta_{A}
$$

Der Fall V3 von Bild 6.30 ist dadurch gekennzeichnet, dass die verformte Wandaxe in einem Wandende tangential zur unverformten Wandaxe verläuft. Das statische system ist statisch einfach unbestimmt. Dementsprechend lässt sich nun die $e_{A} \vartheta_{A}$-Beziehung nicht in gleicher Weise ermitteln wie in den Fällen $V_{1}$ und $V_{2}$. Die $e_{A}-\vartheta_{A}-$ Kurven weisen jedoch qualitativ wiederum einen ähnlichen Verlauf wie in Bild 6.34 auf. Die Wand der Höhe h ist derart in die CDC einzupassen, dass die Wandenden $A$ und $B$ auf die CDC zu liegen kommen und gleichzeitig die CDC im Punkt B tangential zur Geraden AB (= unverformte Wandaxe) verläuft (Bild 6.33 ). 


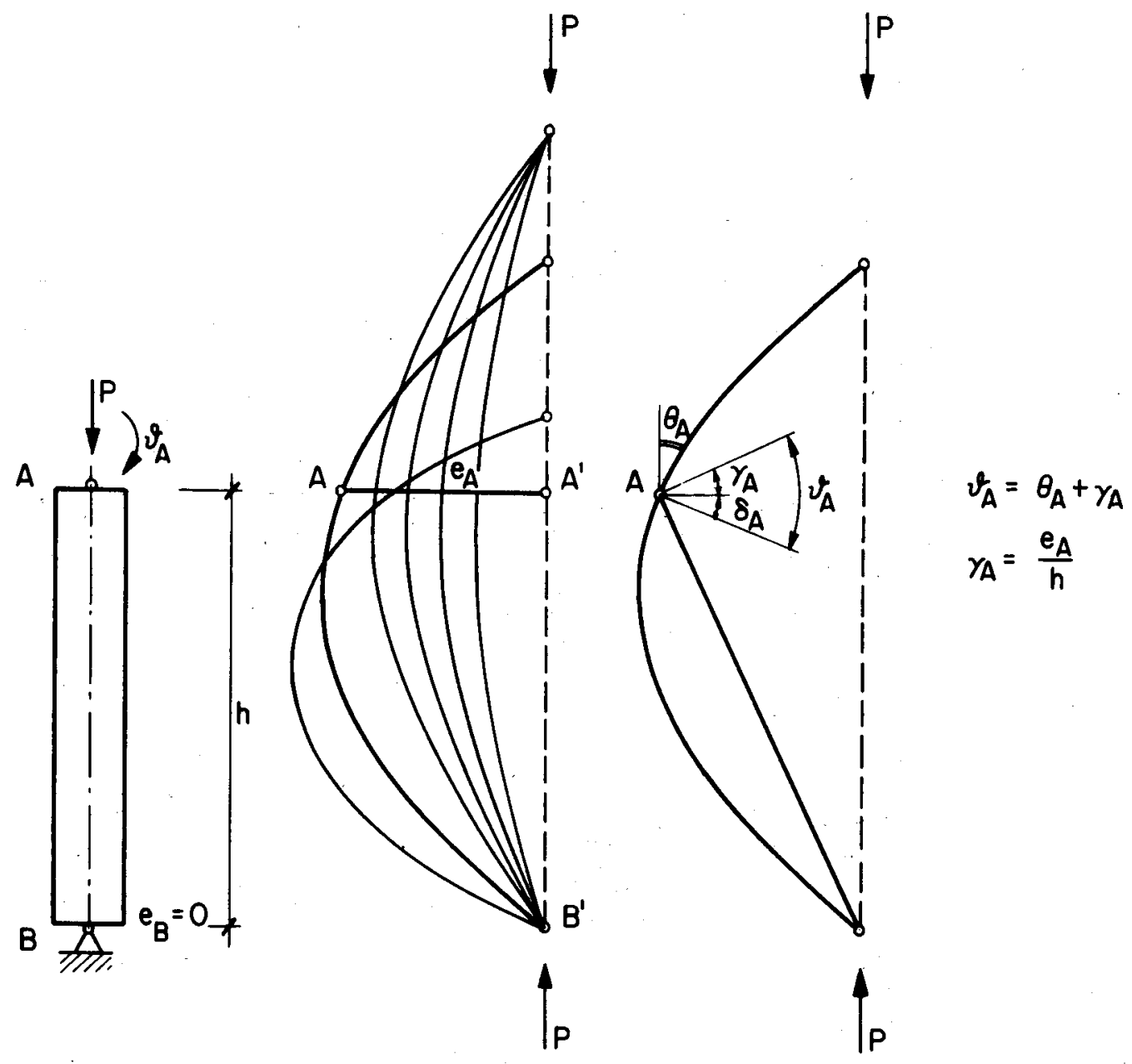

Bild 6.32: Einseitig zentrisch gelagerte Wand (Fall V2)

Die Exzentrizität $e_{A}$ in Punkt $A$ entspricht $A A^{\prime}$ und die dazugehörige Wandendverdrehung $\vartheta_{A}$,kann folgendermassen bestimmt werden:

$$
\vartheta_{A}=\theta_{A}+\frac{e_{A}+e_{B}}{h}
$$

Folgt man dem schematischen Verlauf der $e_{A} \vartheta_{A}-$ Kurve in Bild 6.34 und schliesst Materialversagen vorerst aus, erkennt man, dass die Exzentrizität e $A$ bei zunehmender Verdrehung $\vartheta_{A}$ vorerst wächst und schliesslich ein Maximum (Punkt $M$ ) erreicht. Bei weiterer Verdrehung nimmt $e_{A}$ wieder ab und $e_{A}$ kann sogar negative Werte annehmen. In diesen Fällen stützt sich dann.die Wand zur Erhaltung des Gleichgewichtes auf die benachbarten Decken ab. Schliessilich ergibt sich für die Wandendverdrehung ein kritischer Wert $\vartheta_{A, k r}$ (Punkt S). Bei grösserer Verdrehung würde die Wand ihr Tragvermögen verlieren, ohne dass in einem Schnitt der Wand Materialversagen auftritt. Dieser Sachverhalt wurde bereits bei der Diskussion von Bild 6.18 erläutert. Es handelt sich dabei um einen besonderen Fall eines Stabilitätsversagens bei der Beanspruchungsart $V$. Selbstverständlich wird die Bruchgrenze des Materials in der Regel im Bereich des aufsteigenden (Punkt B) bzw. des abfallenden (Punkt B') Kurvenastes erreicht. 
80

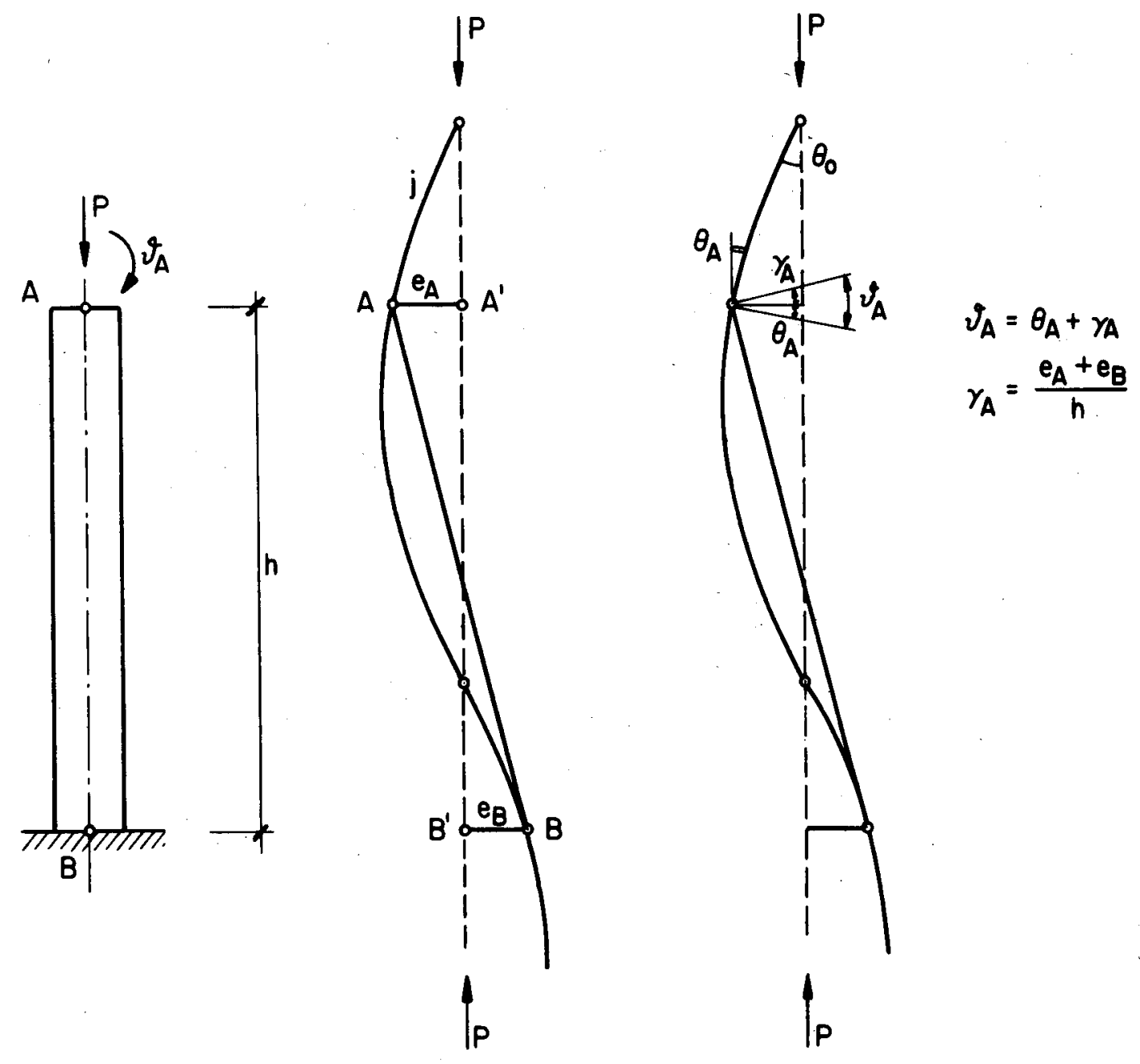

Bild 6.33 : CDC - Belastungsfall $\vee 3$

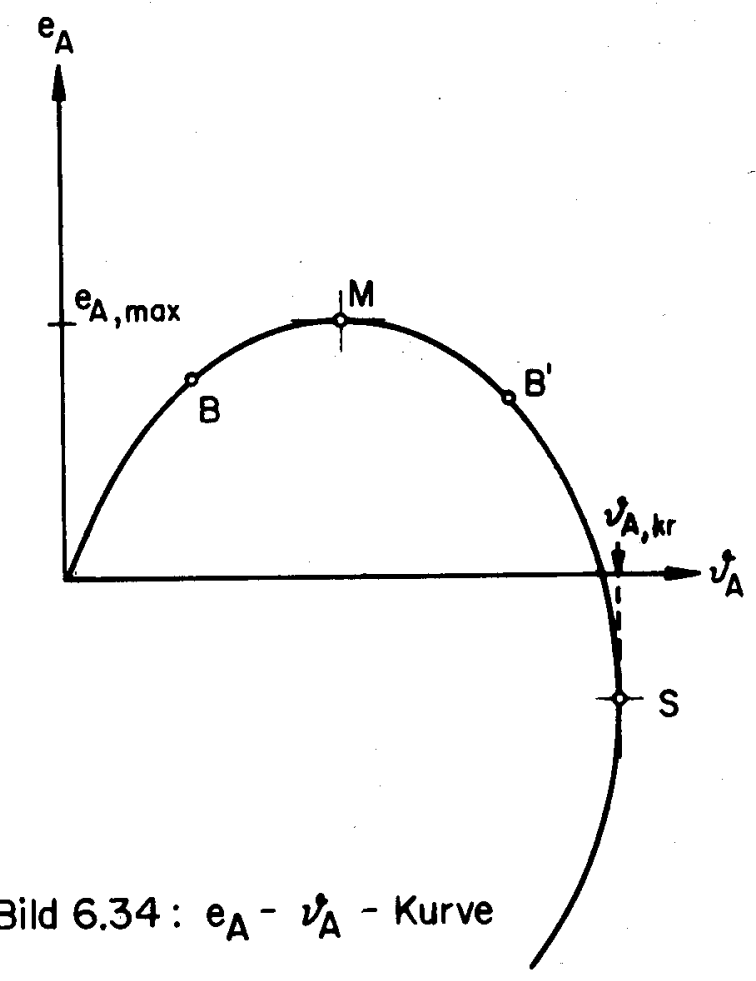


Bei der Berechnung der CDC (vgl. Bild 6.27) können durch die Grösse von e beliebige "Startbedingungen" angenommen werden. Die Annahme der Bruchexzentrizizäz ( $v g l$. Bild 5.11) beispielsweise führt dann in der $e_{A}{ }^{-\vartheta}{ }_{A}-K u r v e ~ z u$ Punkt B.

Beanspruchungsart E

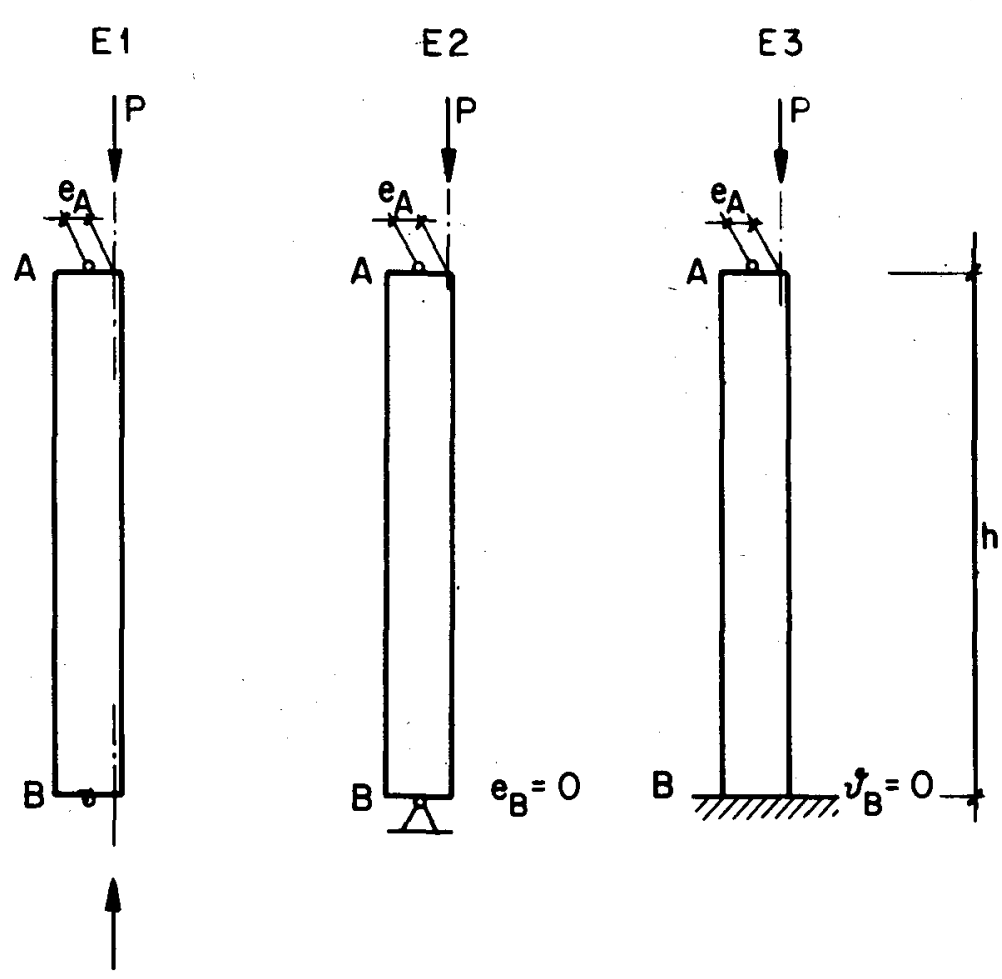

Bild 6.35: 3 Belastungsfälle (Beanspruchungsart E)
Bei der Beanspruchungsart $E$ ist die Exzentrizität durch die Lagerung vorgegeben. In Bild 6.35 sind drei Belastungsfälle dieser Beanspruchungsart dargestelit. Am Beispiel von Fall E1 soll nun das Vorgehen zur Bestimmung der maximalen Exzentrizität erläutert werden.

Nimmt man an, dass die Exzentrizität ${ }^{\mathrm{A}} \mathrm{A}$, max von Bild 6.34 ist, so entspricht die dazugehörige Axiallast $P$ der Traglast $P^{*}$. Die Last kann bei dieser Exz entrizität ${ }^{E_{A} \text {, max }}$ nicht über die Traglast $P^{*}$ hinaus gesteigert werden. Für die Axiallast $P$ kann wiederum eine Schar

von $\operatorname{CDC}$ berechnet werden. Sie ist in Bild 6.36 dargestellt. Verfolgt man für die Wand mit der Schlankheit $\delta$ wiederum den Zusammenhang zwischen der Exzentrizität $e_{A}$ und der Wandendverdrehung $\vartheta_{A}$ (Bild 6.34), so erkennt man, dass sich die maximale Exzentrizität für jene CDC ergibt, die in $A_{k r}$ tangential zur Umhüllenden der Kurvenschar verläuft. Bei der vorhandenen Axiallast $P$ ist also aus Gleichgewichtsgründen keine grössere Exzentrizität möglich. Durch die Umhüllende sind für beliebige Wandschlankheiten $\delta$ die maximalen Exzentrizitäten gegeben. Die Umbüllende ist in Bild 6.36 stark ausgezogen. Sie ist durch das Stoffgesetz und die Axiallast sowie durch die Querschnittsform (Lochung) gegeben. Aus den Umhüllenden verschiedener Axiallasten, sie entsprechen damit den Traglasten $P^{*}$, werden nun für bestimmte Exzentrizitäten e die Schlankheiten ermittelt und in diesem Diagramm den zugehörigen Traglastspannungen $\sigma^{*}$ punktweise zugeordnet. Durch die Verbindung der Punkte erhält man dann ein Traglastspannungsdiagramm (Bild 6.37). Ein solches wurde für das linear elastische Material ohne Zugfestigkeit in Bild 6.17 dargestellt. 


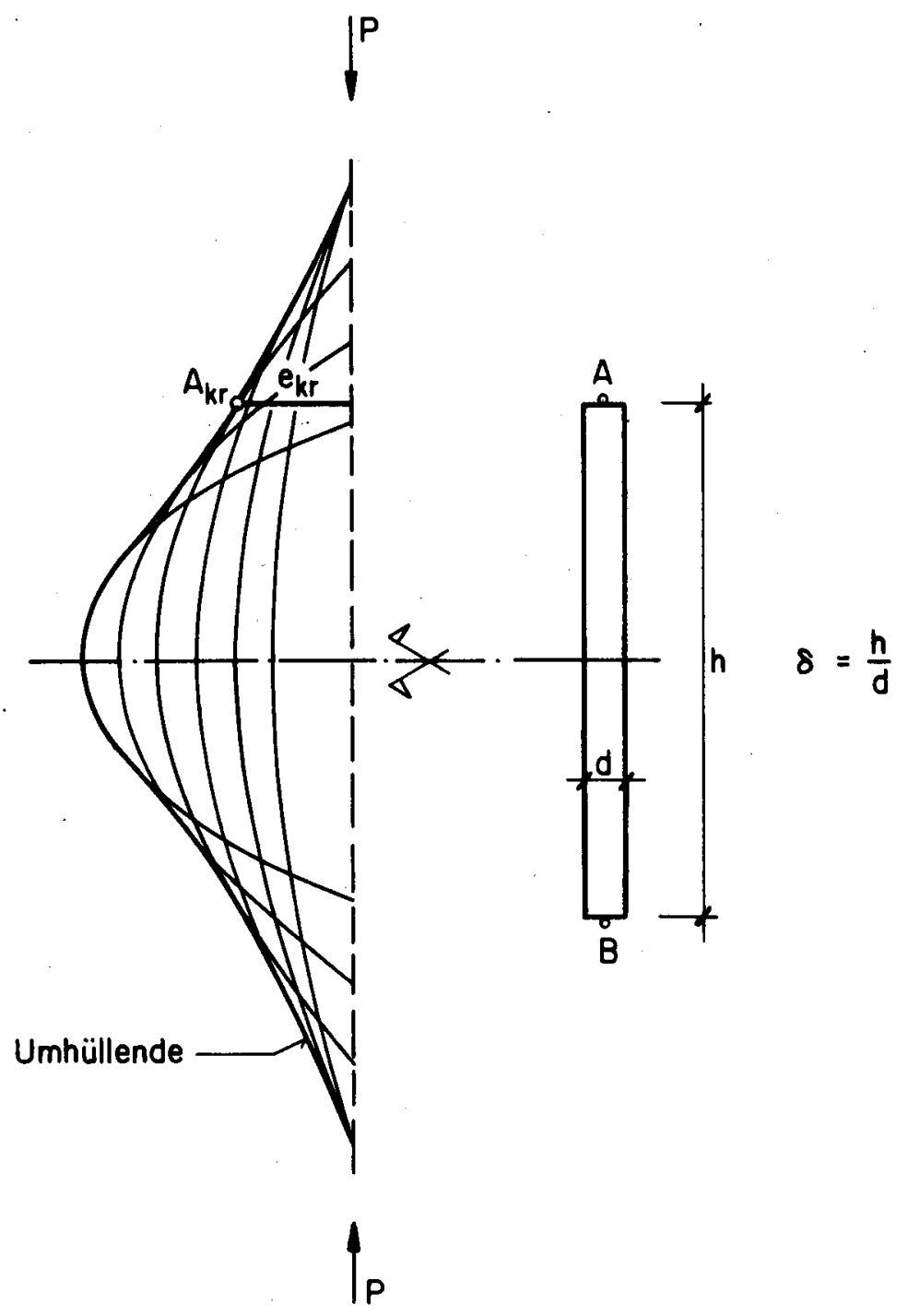

Bild 6.36: CDC-Umhüllende (Belastungsfall E1)

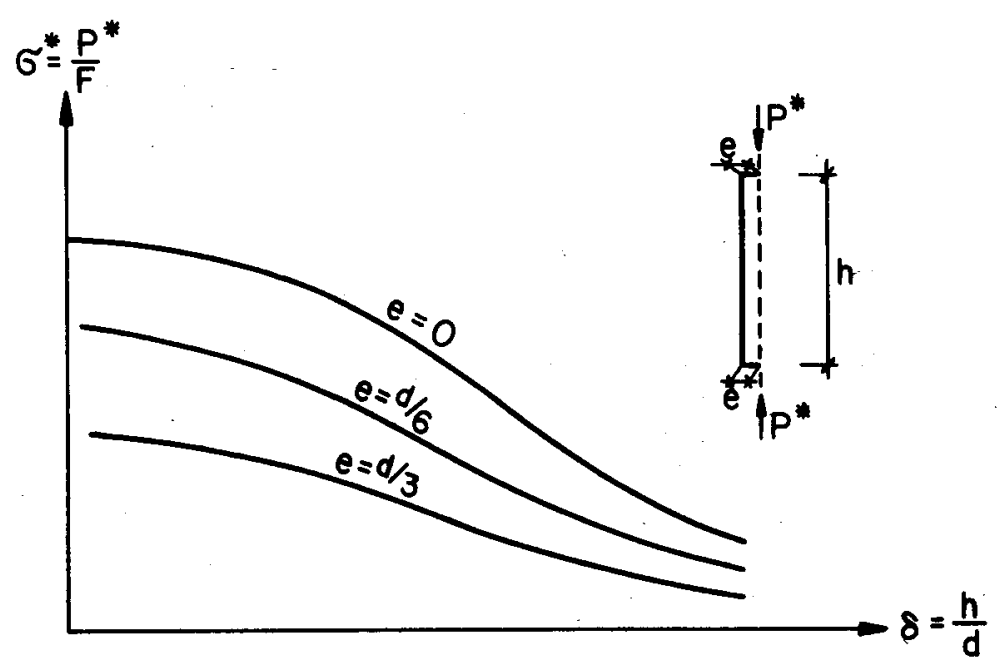

Bild 6.37: Traglastspannung - Schlankheit $\left(\sigma^{*}-\delta\right)$ 
Für den Belastungsfall E2 ist die Umhüllende an die Schar der verschobenen $\operatorname{COC}$ in Bild 6.32 zu bestimmen (Bild 6.38).

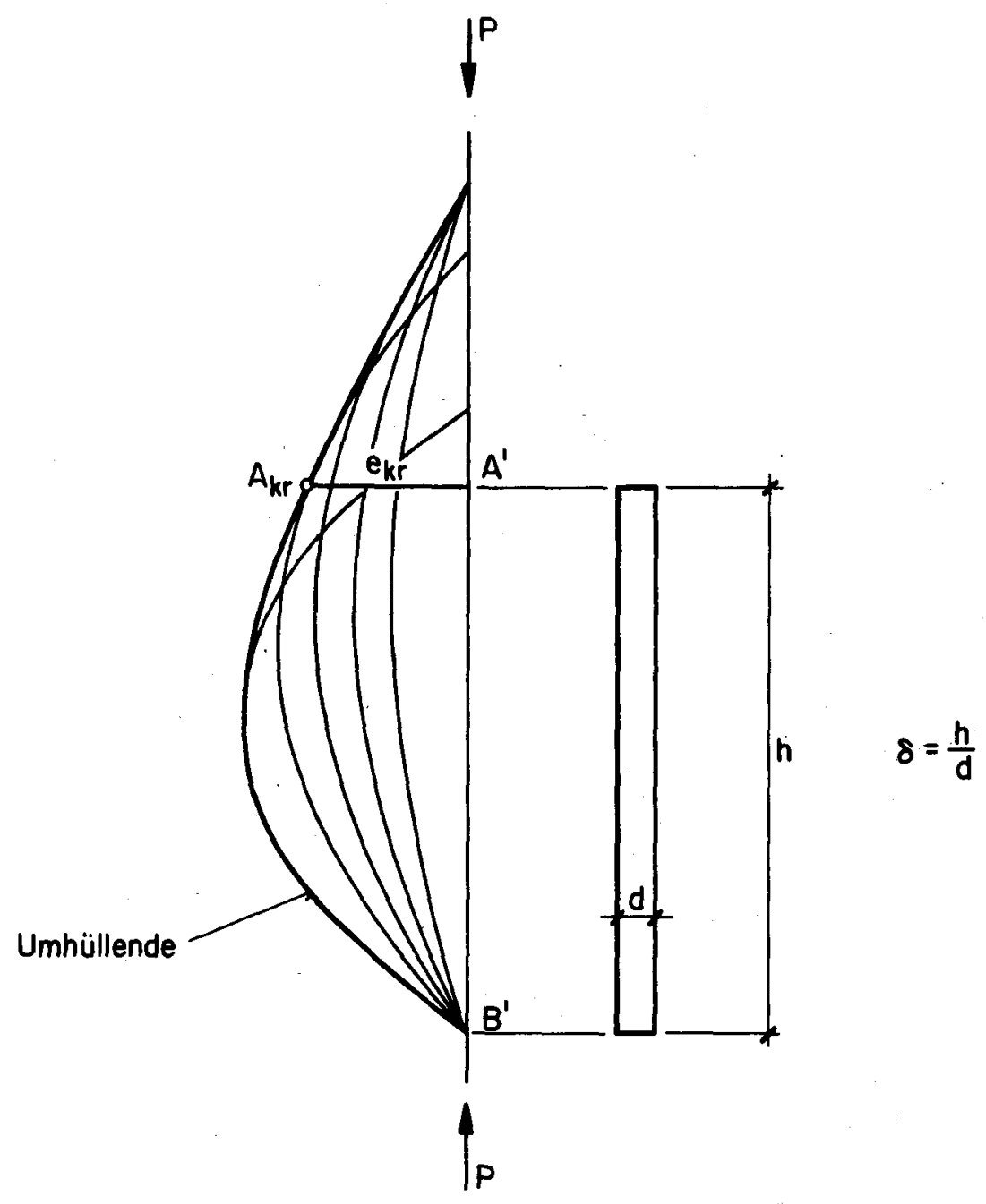

Bild 6.38: CDC-Umhüllende (Belastungsfall E2)

\subsection{Starrkörper-Federmodel 1}

In den Rotationsversuchen [7] und [8] wurde festgestellt, dass sich bei der Verdrehung des unteren Wandendes in den Fugen deutliche Knicke einstellten. Die Verformungsanteile in den Fugen waren bedeutend grässer als in den Mauersteinen. Bei kleiner Axiallast und grosser Deckenverdrehung ergab sich ein klaffender Riss bzw. starker Knick in einer Mörtelfuge. Demgegenüber waren diese konzentrierten Winkeländerungen in den Fügen bei grossen Axiallasten gleichmässiger über die Höhe verteilt. Diese Beobachtungen legten nahe, das Verhalten der Maverwerkswände mit einem Starrkörper-Federmodell (Bild 6.39) theoretisch zu beschreiben. Es besteht aus Drehfedern und starren Verbindungselementen (Starrkörper) der Länge $\Delta x$. Die Länge $\Delta x$ entspricht dabei gerade der Summe von Steinhöhe $h_{S}$ und Fugendicke $h_{M}$. 

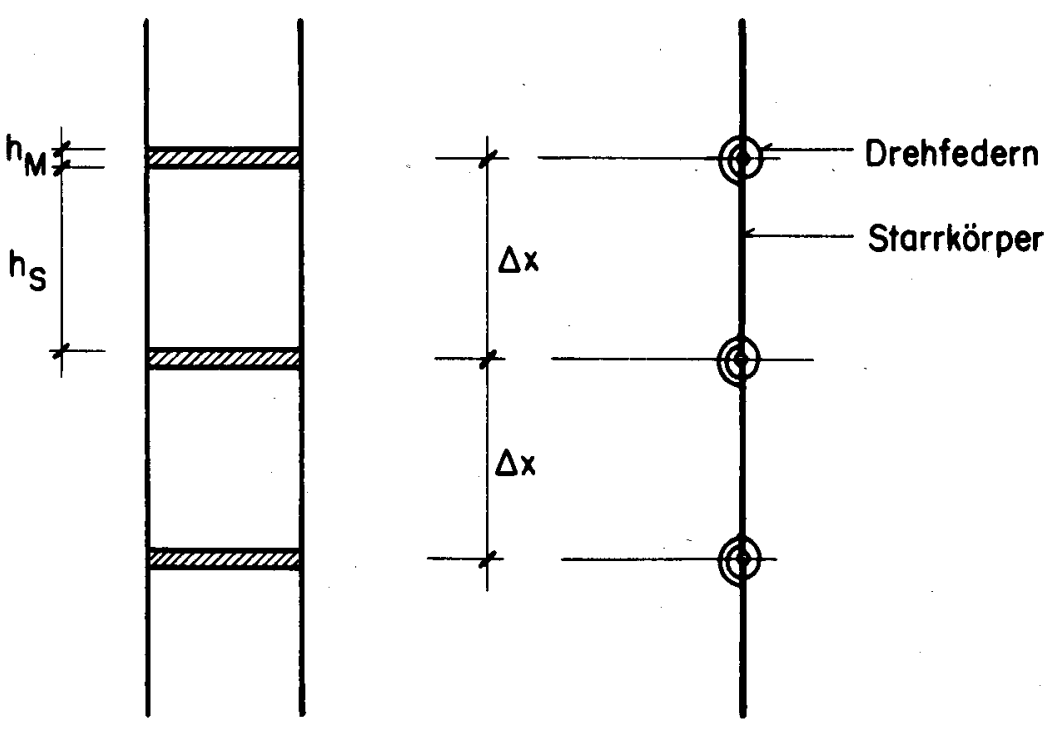

\section{Bild 6.39: Modellbildung}

In 6.3.2 wurde das Starrkörper-Federmodell als Verfeinerung des einfachen Federmodells erläutert und der Einfluss der Länge $\Delta x$ auf den Verlauf der Exzentrizitäts-Verdrehungs-Kurve (Bild 6.23) dargestellt. Rechnerisch kann es mit der in den Abschnitten 6.3.3 und 6.3.4 beschriebenen Methode der CDC behandelt werden. Der sich dabei ergebende grosse Rechenwand kann nur mit einer Rechenmaschine bewältigt werden, so dass im Rahmen der vorliegenden Arbeit ein entsprechendes Computerprogramm entwickelt wurde (vgl. 6.3.1).

\subsubsection{Charakteristik der Drehfedern}

Bei der Berechnung der CDC (Bild 6.28) wurden Exzentrizitäts-Krümmungs-Beziehungen verwendet. Ausgehend von einer bekannten Exzentrizität $e_{i}$ im Schnitt $i$ konnte damit unter der zusätzlichen Annahme konstanter Krümmung im Integrationsabschnitt $\Delta x$ die Exzentrizität $e_{i+1}$ im Schnitt $i+1$ ermittelt werden. Im Gegensatz dazu verlaufen die CDC beim Starrkörper-Feder-Modell innerhalb des Integrationsabschnittes $\Delta x$ geradlinig (Krümmung $=0$ ) und weisen in den Verbindungsstellen der Starrkörper bzw. in den Drehfedern konzentrierte Winkeländerungen (Knicke) auf. Diese Winkeländerungen sind durch die Charakteristik der Drehfedern bestimmt. Sie kann aus den experimentell bzw. rechnerisch ermittelten Exzentrizitäts-Krümmungs-Kurven bestimmt werden.

In Bild 6.40 ist schematisch der Exzentrizitätsverlauf entlang der Axe einer Wand dargestellt. Aus der e- $\Phi-B e z i e h u n g$ lässt sich dann der entsprechende Krümmungsverlauf angeben. Bei den Krümmungswerten der e- $\Phi$-Kurven handelt es sich um Mittelwerte (vgl. Abschn. 5.1). Im Starrkörper-Feder-Modell treten nun anstelle der Krümmungen $\Phi$ in den Drehfedern Rotationen $\varphi$ auf. Diese entsprechen dem Integral der Krümmungen im Abschnitt der Länge $\Delta x$, wobei die Drehfeder in der Mitte des Abschnittes $\Delta x$ liegt. Unter der Annahme, dass die Krümmungen im Mittel der Krümmung $\Phi$ an der Stelle der Feder entspricht, er- 
Mauerwerkwand: Exzentrizität : Krümmung: Starrkörper-Federn:

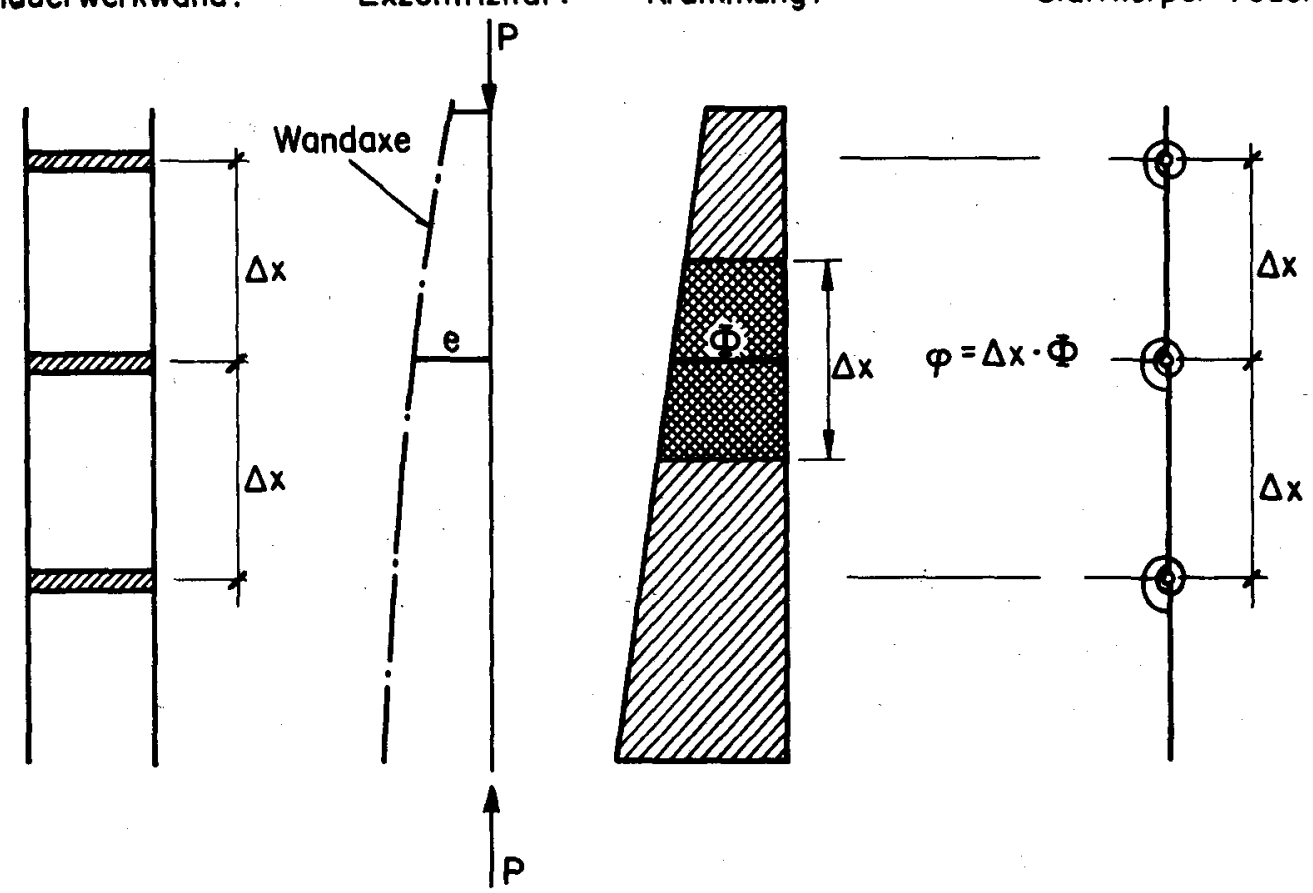

Bild 6.40: Ermittlung der Federcharakteristik

gibt sich für den Zusammenhang zwischen der Krümmung und der Verdrehung die einfache Beziehung

$\varphi=\Delta x \cdot \Phi$

Um aus den e- $\Phi-K u r v e n$ die Federcharakteristiken zu bestimmen, sind demnach die Abszissenwerte (Krümmungen) mit $\Delta x$ zu multiplizieren. Die Charakteristik. der Drehfedern wird also durch eine Beziehung zwischen der Exzentrizität e und der dabei sich ergebenden Verdrehung der Feder beschrieben. Wie die e- $\Phi-$ Kurven sind auch die e- $\varphi$-Kurven Funktionen von P.

\subsubsection{Vergleich mit experimentellen Resultaten}

Durch den Vergleich mit experimentellen Resultaten soll gezeigt werden, inwiefern das Starkörper-Feder-Modell das tatsächliche Trag- und Bruchverhalten von Backsteinmauerwerkswänden zu beschreiben vermag. Vergleichsbasis bilden die in den Versuchen beobachteten Exzentrizität-Verdrehungs-Kurven. In den Versuchsberichten [7] und [8] sind solche ausführlich dargestellt.

Es handelt sich dabei um den Zusammenhang zwischen der Exzentrizität e der Axiallast auf der Höhe des unteren Linienkipplagers (Bild 5.1) und der Verdrehung $\vartheta_{D}$ der Betonplatte. Die Exzentrizität $e_{B}$ wurde mit Gl. (5.1) bestimmt, und die Verdrehung $\vartheta_{D}$ der Betonplatte wurde mit einem Klinometer gemessen. Sie übersteigt die Wandendverdrehung, die in den bisherigen Untersuchungen betrachtet wurde, um die Rotation in der Fuge zwischen Wand und Betonplatte. 


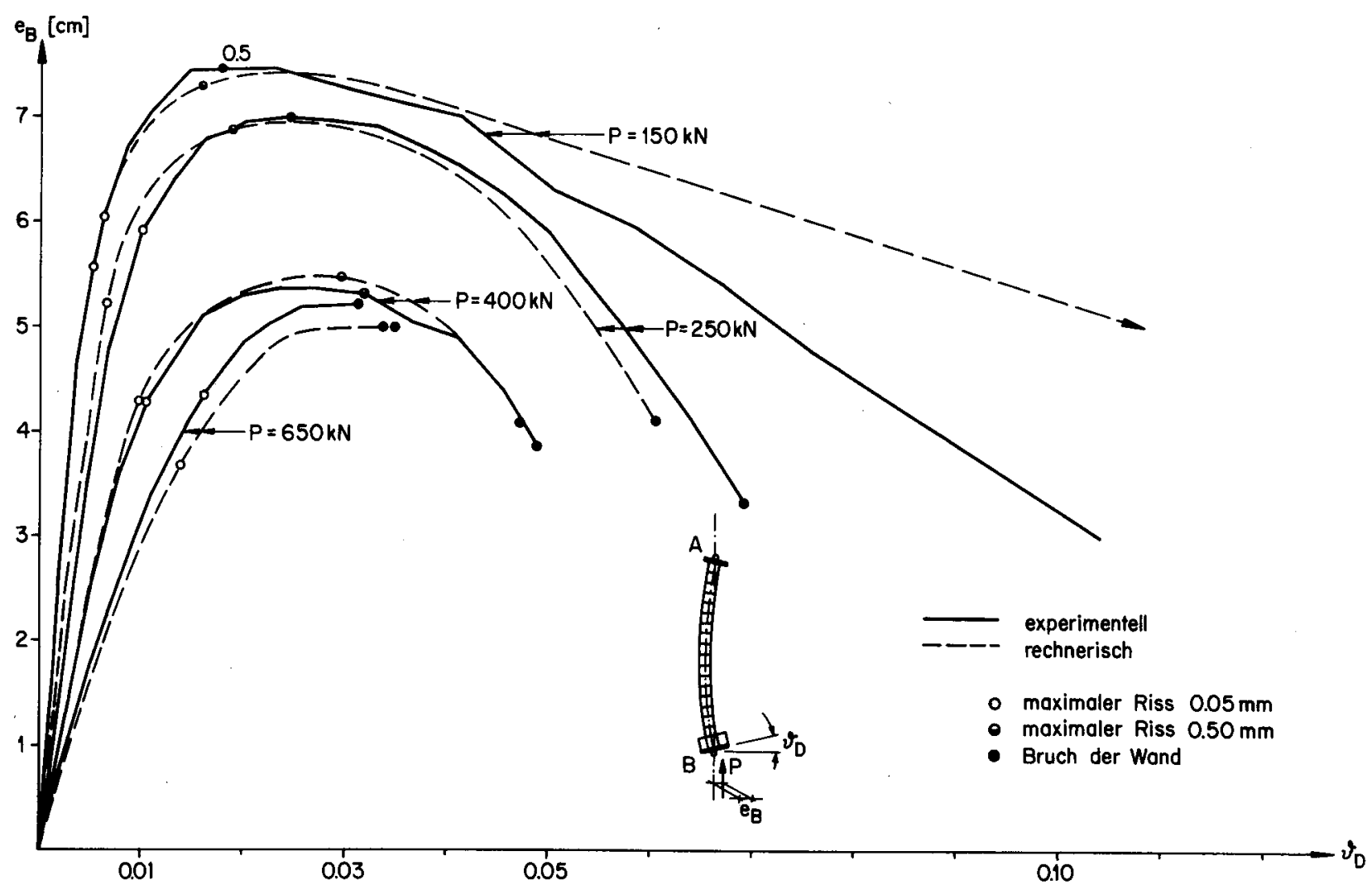

Bild 6.41: Exzentrizitöts -Verdrehungs-Kurven (experimentell-rechnerisch)

In Bild 6.41 sind für Axiallasten $P=150,250,400$ und $650 \mathrm{kN}$ experimentelle Exzentrizitäts-Verdrehungs-Kurven dargestellt. Sie sind stark ausgezogen. Diese Beziehungen wurden an $15 \mathrm{~cm}$ dicken und $90 \mathrm{~cm}$ langen Wänden aus $\mathrm{BH}-$ Steinen und Zementmörtel bestimmt [7]. Nach einem fast linear ansteigenden Ast flachen die Kurven ab und überschreiten mit Ausnahme der Kurve für $P=$ $650 \mathrm{kN}$ ein Maximum. Die Bruchverdrehung wird dann im Bereich des abfallenden Kurvenabschnittes erreicht. Bei der Kurve für $P=650 \mathrm{kN}$ stellt sich die Bruchverdrehung ungefähr bei der maximalen Exzentrizität ein. In Bild 6.34 wurde der generelle Verlauf solcher Kurven diskutiert. Bei der Verdrehung der Betonplatte ergaben sich in den Lagerfugen sowie zwischen dem unteren Wandende und der Betonplatte Risse. Deren Grössen wurden nach jeder schrittweisen Zunahme der Verdrehung der Betonplatte beobachtet.

In den Kurven sind die Punkte, bei denen maximale Risse von 0.05 und $0.5 \mathrm{~mm}$ auftreten, durch leere und halbgefüllte Kreise dargestellt. Bei einer Axiallast von $400 \mathrm{kN}$ ergab sich beispielsweise ein maximaler Riss von $0.05 \mathrm{~mm}$ bei einer Verdrehung $\vartheta_{D}$ von 0.0105 und ein solcher von $0.5 \mathrm{~mm}$ bei $\vartheta_{D}=0.032$.

In Bild 6.42 sind auf der Ordinate die Axiallasten und auf der Abszisse diejenigen Verdrehungen, bei denen sich maximale Risse von 0.05 und $0.5 \mathrm{~mm}$ ergaben bzw, der Bruch erfolgte, durch Punkte dargestellt. Es wurden dabei sämtliche Versuche, die an $15 \mathrm{~cm}-M B H C$ (vgl. Anhang A1) gleicher Provenienz und Zementmörtel durchgeführt wurden, berücksichtigt. 


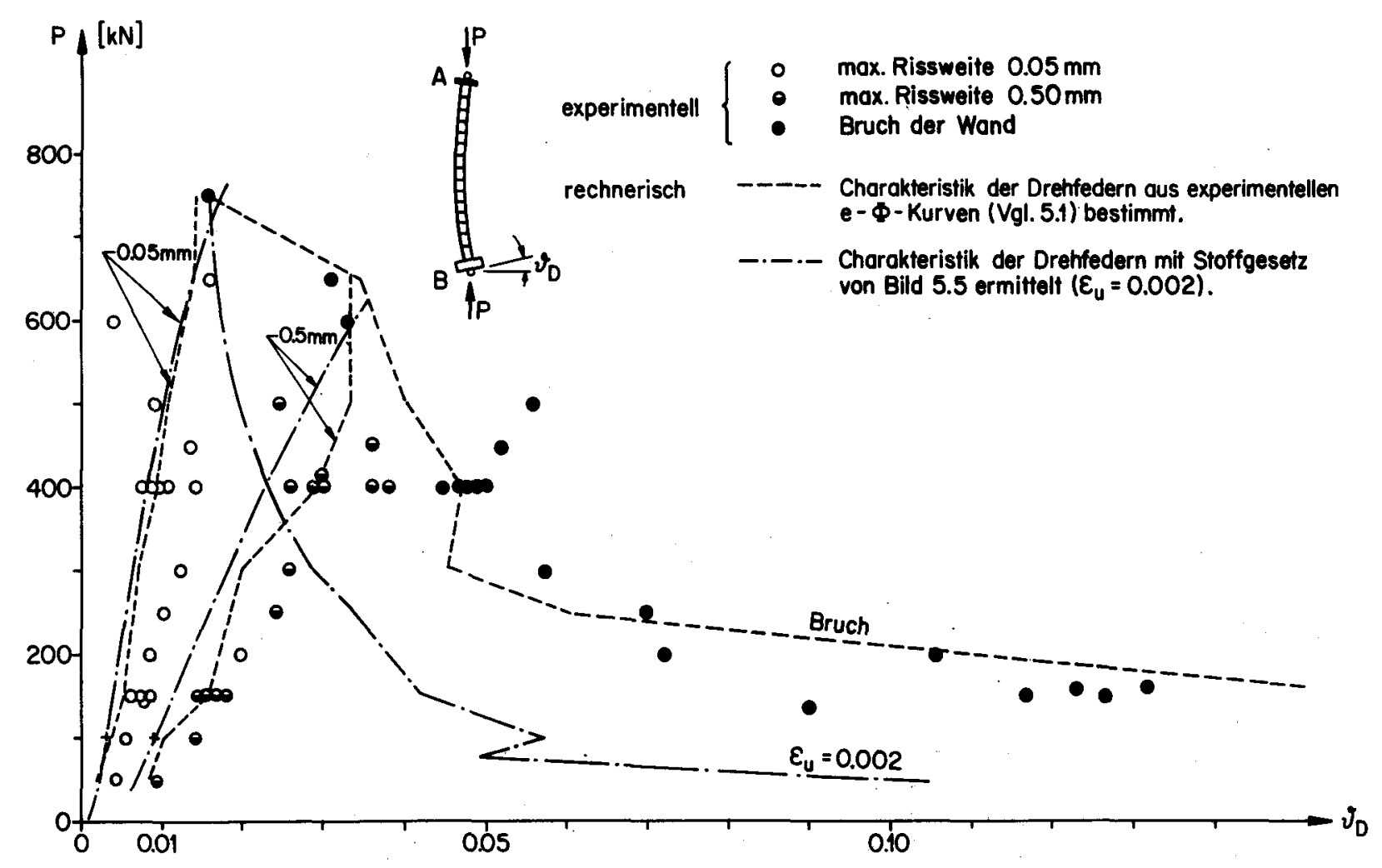

Bild 6.42: Axiallast - Verdrehung für Riss und Bruch (rechnerisch - experimentell)

Es zeigt sich in dieser Darstellung deutlich, dass die Bruchverdrehung mit wachsender Axiallast abnimmt (schwarze Punkte). Demgegenüber sind bei zunehmender Axiallast stets grössere Verdrehungen erforderlich, damit Risse von 0.05 und $0.5 \mathrm{~mm}$ in den Lagerfugen entstehen. Der Grund liegt darin, dass die Rissöffnungen bei grossen Axiallasten gleichmässiger über die Wandhöhe verteilt sind als bei kleinen Lasten.

Die rechnerischen $e_{B}^{-\vartheta}{ }_{D}^{-K u r v e n(B i l d ~ 6.4) ~ s o w i e ~ d i e ~ r e c h n e r i s c h e n ~ K u r v e n ~ i n ~}$ Bild 6.42 wurden mit dem Starrkörper-Feder-Modell bzw. mit dem Computerprogramm ermittelt. Die Lagerungsbedingungen der Wand entsprachen grundsätzlich dem Fall V2 der Beanspruchungsart $V(B i l d 6.30)$. Die Abweichungen, die sich durch die Stahlplatten an beiden Wandenden sowie durch den Umstand, dass anstelle der Wandendverdrehung die Verdrehung der Betonplatte zu ermitteln war, wurden durch entsprechende Aenderungen im Computerprogramm berücksichtigt.

Die Charakteristiken der Drehfedern (vgl. 6.4.1) wurden aus den experimentell bestimmten e- $\Phi$-Kurven ( $v g l$. 5.1) berechnet. Die Uebereinstimmung der rechnerischen und experimentellen Kurven in Bild 6.41 ist befriedigend. Die Kurven für eine Axiallast $P$ von $150 \mathrm{kN}$ weisen im Bereich des abfallenden Kurvenabschnittes eine grössere Abweichung auf. Die rechnerische Exzentrizität ist bei gleicher Verdrehung stets grösser als die experimentell bestimmte Exzentrizität. Währenddem sich in den fünf Versuchen mit der Axiallast von $150 \mathrm{kN}$ die Verdrehung in der Fuge oberhalb der ersten Steinreihe bzw. zweiten Steinlage konzentrierte, ergab sich dieser "Knick" in der Rechnung in der Fuge zwischen Wand und Betonplatte. 
In Bild 6.42 sind nebst den experimentellen Punkten je zwei rechnerische Kurven für Risse von 0.05 und $0.5 \mathrm{~mm}$ bzw. für den Bruch dargestellt. Die gestrichelten Kurven korrespondieren mit denjenigen von Bild 6.41. Diesen Kur-

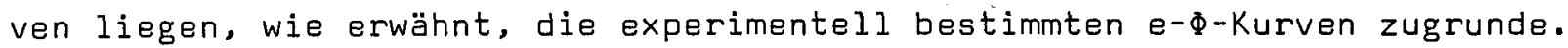
Demgegenüber verlaufen die strichpunktierten Kurven kontinuierlicher. Den Berechnungen dieser Kurven liegen die mit dem Stoffgesetz von Bild 5.5 er-

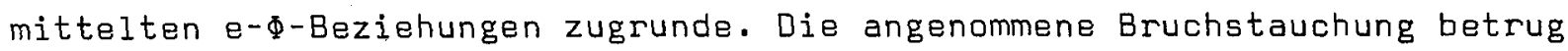
im vorliegenden Fall 0.002 . Die gestrichelten Kurven folgen besser dem Verlauf der experimentellen Punktwolken. Grössere Abweichungen liegen insbesondere bei den Bruchkurven vor. Entlang der strichpunktierten Kurve sind die Bruchverdrehungen viel geringer als die gemessenen bzw. diejenigen, die sich aus dem Verlauf der gestrichelten Kurve ergeben. Der Grund liegt darin, dass die rechnerische Bruchstauchung $\varepsilon_{u}$ mit 0.002 ein vorsichtiger wert ist. Die Darstellung in Bild 5.9 zeigt, dass insbesondere im Bereich von kleinen Axiallasten die experimentellen Bruchstauchungen den Wert von 0.004 deutlich übersteigen. Bei grosser Axiallast $(P=750 \mathrm{kN})$ beträgt die Bruchstauchung ca. 0.002. Auf diesem Lastniveau liegen die rechnerischen und experimentellen Bruchverdrehungen denn auch nahe beieinander.

\subsubsection{Folgerungen}

Der Vergleich von rechnerischen und experimentellen Resultaten zeigt eine gute Uebereinstimmung. Das Tragverhalten kann demnach rechnerisch mit dem Starrkörper-Feder-Modell beschrieben werden. Es darf angenommen werden, dass dieses rechnerische Verfahren auch für andere Mauerwerksarten, beispielsweise für Kalksandsteinmauerwerk verwendet werden kann und eine gute Uebereinstimmung mit experimentellen Resultaten liefern wird.

\subsection{Einfluss der Zugfestigkeit und der Lochung auf das Trag- und Bruchverhalten}

In Kapitel 4 wurde der Einfluss der Zugfestigkeit und der Lochung auf der Grundlage eines linear elastischen Materials in bezug auf das Krümmungsverhalten und die Interaktion M-P untersucht (Abschnitte 4.3 und 4.5 ).

Im Folgenden sollen nun die Auswirkungen der obigen Einflussgrössen auf die Riss- und Bruchverdrehung von Mauerwerkswänden am Beispiel der symmetrisch belasteten Wand (Fall V1, Bild 6.43) diskutiert werden.

Die in den Bildern 6.45 und 6.46 dargestellten Kurven wurden mit dem Starrkörper-Feder-Modell (bzw. mit dem Computerprogramm) berechnet. Die Charakteristiken der Drehfedern wurden wie folgt bestimmt: Auf der Grundlage des linear elastischen Stoffgesetzes und unter Berücksichtigung der Zugfestigkeit bzw. der Lochung wurden e- $\Phi$-Beziehungen (Bilder 4.10 und 4.17) ermittelt. Aus diesen e- $\Phi$-Kurven konnten analog 6.4.1 die Charakteristiken e- $\varphi$ berechnet werden. 


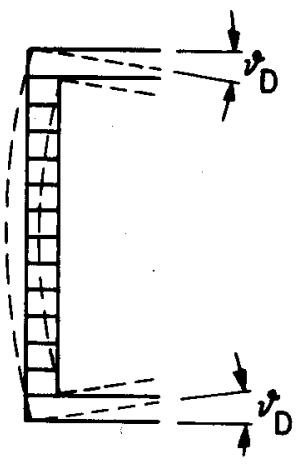

Bild 6.43: Symmetrische Wandbelastung

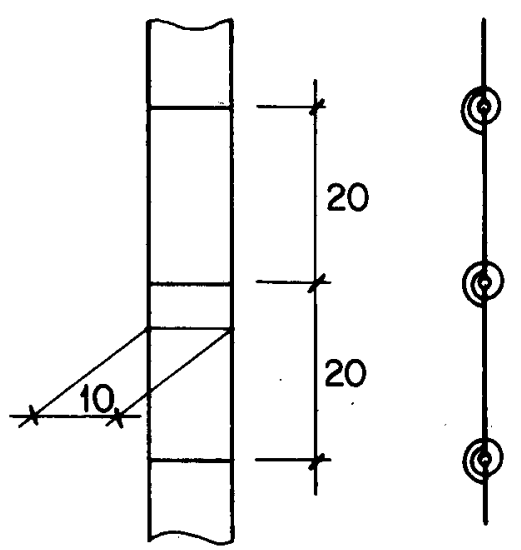

Bild 6.44 : Starrkörper-Feder-Modell

\subsubsection{Einfluss der Zugfestigkeit $\sigma_{z u}$}

In Abschnitt 4.3 wurde $\sigma_{z u}$ auf die Bezugsspannung $\sigma_{b}$ bezogen:

$$
\sigma_{z u}=-\zeta \cdot \sigma_{b}
$$

Für $\zeta$-Werte von 0 und 0.10 sind in den Bildern 6.45 a,b,c (Schlankheiten $\delta=10,30$ und 50) die mittleren Spannungen in Abhängigkeit der Verdrehungen $\vartheta_{D}$, bei denen im Querschnitt in Wandmitte die Randspannung $\sigma_{r}$ gleich der Bezugsspannung $\sigma_{b}$ bzw. die Randdehnung $\varepsilon_{r z}$ gleich $-0.1 \cdot \varepsilon_{b}$ ist, aufgetragen. Nimmt man an, dass die Bezugsspannung $\sigma_{b} b z w$. die Bezugsstauchung $\varepsilon_{b}$ gerade der Bruchspannung $\sigma_{u}$ bzw. der Bruchstauchung $\varepsilon_{u}=0.002$ entspricht, so ergeben sich bei $=-0.1 \cdot \varepsilon_{b}=-0.0002$ und einer Starrkörper-Länge $\Delta x=200 \mathrm{~mm}$ nach Gl. (5.5) Risse der Grösse $r=0.04 \mathrm{~mm}$, und die Verdrehungen, die zu $\sigma_{r}=\sigma_{b}=\sigma_{u}$ führen, können als Bruchverdrehungen bezeichnet werden.

Die Kurvenverläufe in Bild 6.45 zeigen, dass die Zugfestigkeit auf die Grösse der Bruchverdrehung der Deckenauflager keinen grossen Einfluss ausübt. Solange im Querschnitt in Wandmitte alle Fasern gestaucht sind, spielt die Zugfestigkeit ohnehin keine Rolle. Bei geringen Axiallasten vermindert sich die Bruchverdrehung mit zunehmender Zugfestigkeit. 


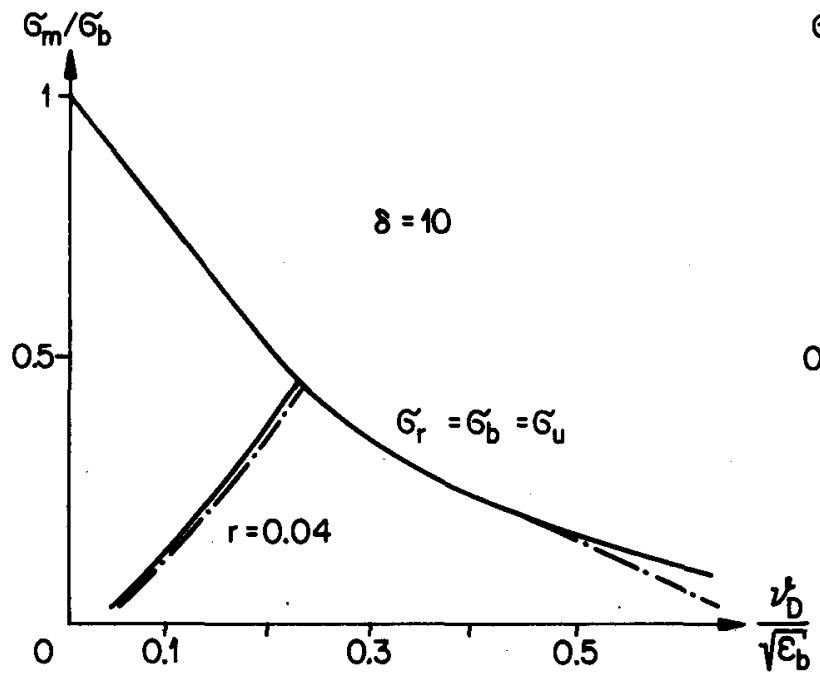

a) Wondschlankheit $8=10$

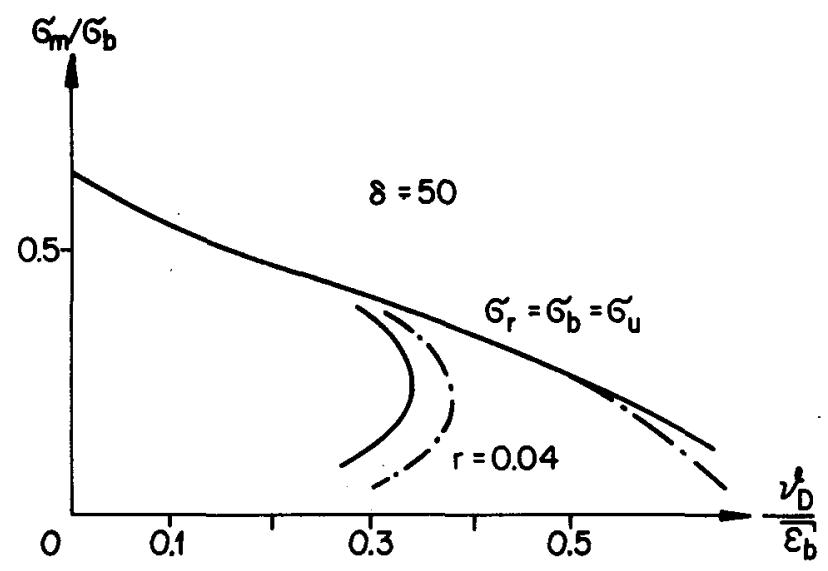

c) Wandschlankheit $8=50$

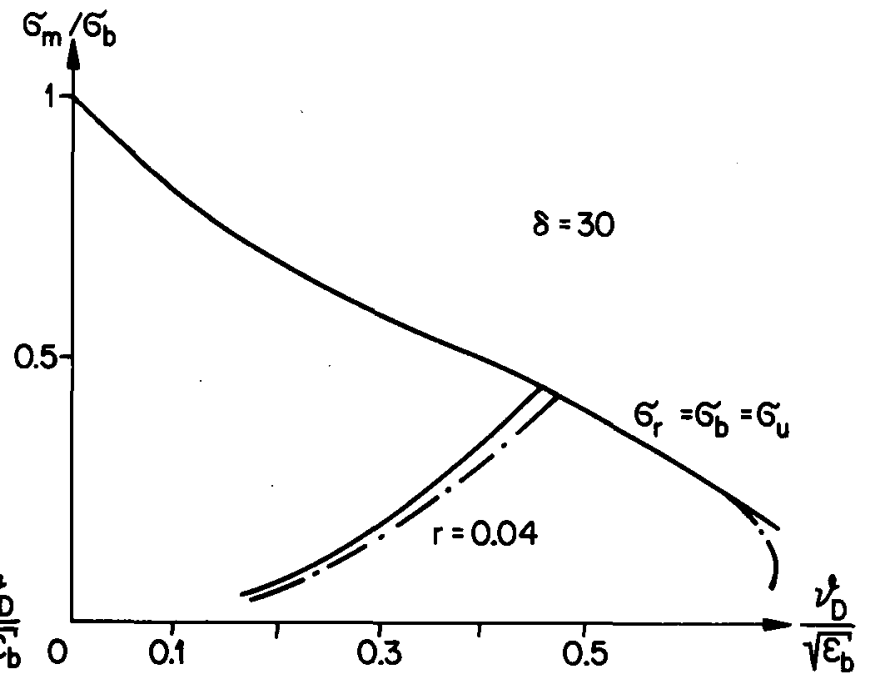

b) Wandschlankheit $8=30$

\section{Bild 6.45: $\sigma_{m}-\vartheta_{D}$ : Einfluss der Zugfestigkeit}

Von grösserer Bedeutung hingegen ist die Zugfestigkeit für die Rissbildung. Risse von $0.04 \mathrm{~mm}$ ergeben sich ohne Zugfestigkeit bei kleineren Verdrehungen des Deckenauflagers als bei vorhandener Zugfestigkeit $\sigma_{z u}=-0.1 \cdot \sigma_{b}$. Dieser Einfluss der Zugfestigkeit nimmt mit grösserer Schlankheit $\delta$ zu.

Im Backstein- und Kalksandstein-Mauerwerk ist die Zugfestigkeit gering und demzufolge für obige Rissbetrachtungen nicht von grosser Bedeutung. Bei geklebten Fugen (z.B. Gasbeton-Mauerwerk) kännte die Zugfestigkeit hingegen grössere Werte aufweisen und wäre dementsprechend in Rechnung zu stellen.

\subsubsection{Einfluss der Lochung}

In Abschnitt 4.5 wurde der Einfluss der Lochung auf das e- $\Phi$-Verhalten sowie auf die Interaktion $M-P$ auf der Grundlage von zwei extremen Lochungen $A$ und $B$ (Bild 4.16) untersucht.

In den Bildern 6.46 a,b,c (für $\delta=10,30$ und 50) sind die mittleren Spannungen $\sigma_{m}$ in Abhängigkeit der Verdrehungen $\vartheta_{D}$, bei denen im Querschnitt in Wandmitte Risse der Grösse $r=0.04 \mathrm{~mm}$ bzw. Materialbruch $\left(\sigma_{r}=\sigma_{b}=\sigma_{u}\right)$ erfolgen (vgl. Abschn. 6.5.1), für den Vollquerschnitt $(\alpha=0.5)$ sowie für Steine mit 


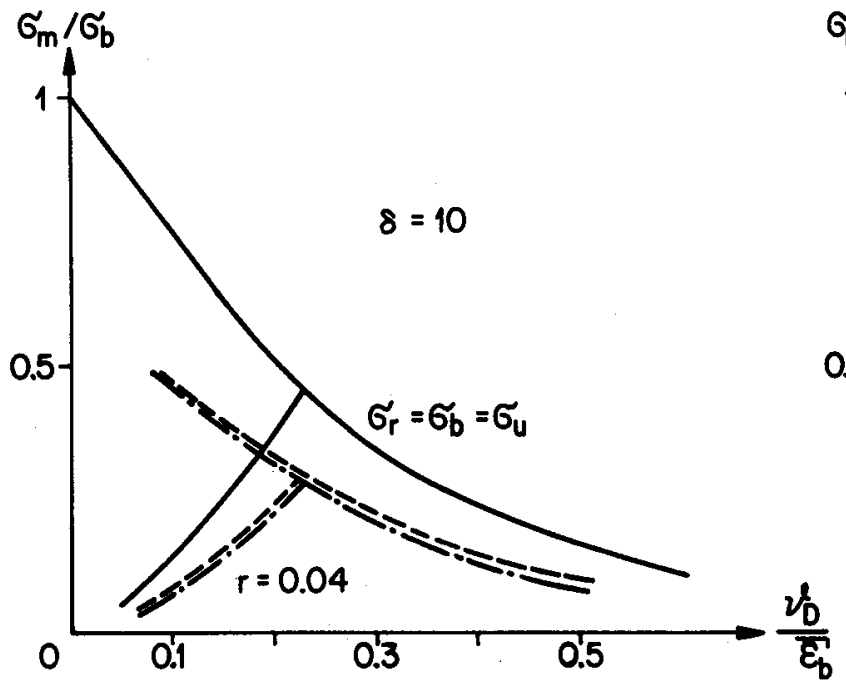

a) Wandschlankheit $8=40$

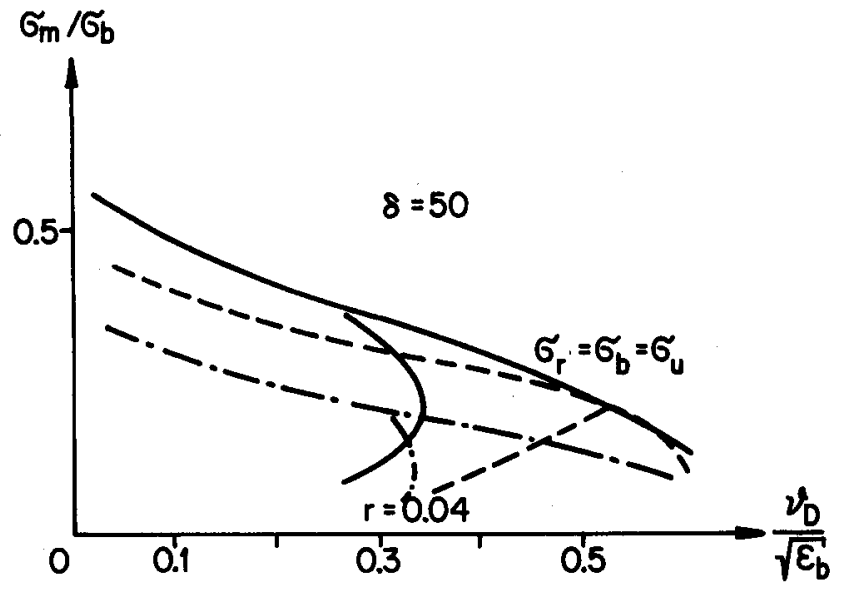

c) Wandschlankheit $8=50$

\section{Legende :}

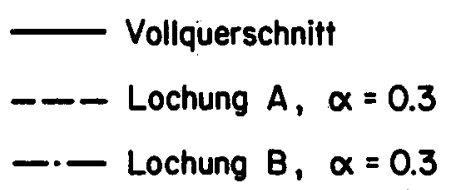

Bild 6.46: $\sigma_{m}-\nu_{D}$ : Einfluss der Lochung

Lochung $A$ und Lochung $B$ dargestellt. a beträgt in diesen beiden Fällen 0.3 .

Die Kurvenverläufe in Bild 6.46 zeigen, dass die Lochung einen markanten Einfluss auf die Bruch- und Rissverdrehungen hat. Der mit abnehmendem $\alpha$ unabhängig von der Lochung $A$ oder $B$ zunehmende Lochflächenanteil Lo führt bei gleicher Axiallast zu grösseren Nettospannungen. Dieser Einfluss der Lochung ist bedeutend grösser als derjenige, der sich aus der unterschiedlichen Materialverteilung von Lochung $A$ und $B$ ergibt; d.h. die Anordnung bzw. die Form der Löcher ist wesentlich weniger bedeutsam als der Lochflächenanteil. 


\section{Wand - Decken - System}

\subsection{Ueberblick}

In Kapitel 6 wurde das Tragverhalten der Mauerwerkswand theoretisch untersucht und mit experimentellen Ergebnissen überprüft. Wie bereits erwähnt, stellt die in Bild 6.1 dargestellte Konstruktion - Mauerwerkswände und daring eingebundene Stahlbetondecken - im Hochbau die übliche Bauweise dar. Einerseits erfahren die Decken durch die Wände eine Einspannung und andererseits werden den wänden infolge von Deckenlasten sowie infolge von Kriech-, Schwind- und Temperatureinflüssen Verformungen aufgezwungen. Die resultierenden Beanspruchungen der Wände und Decken ergeben sich aus ihrem Zusammenwirken.

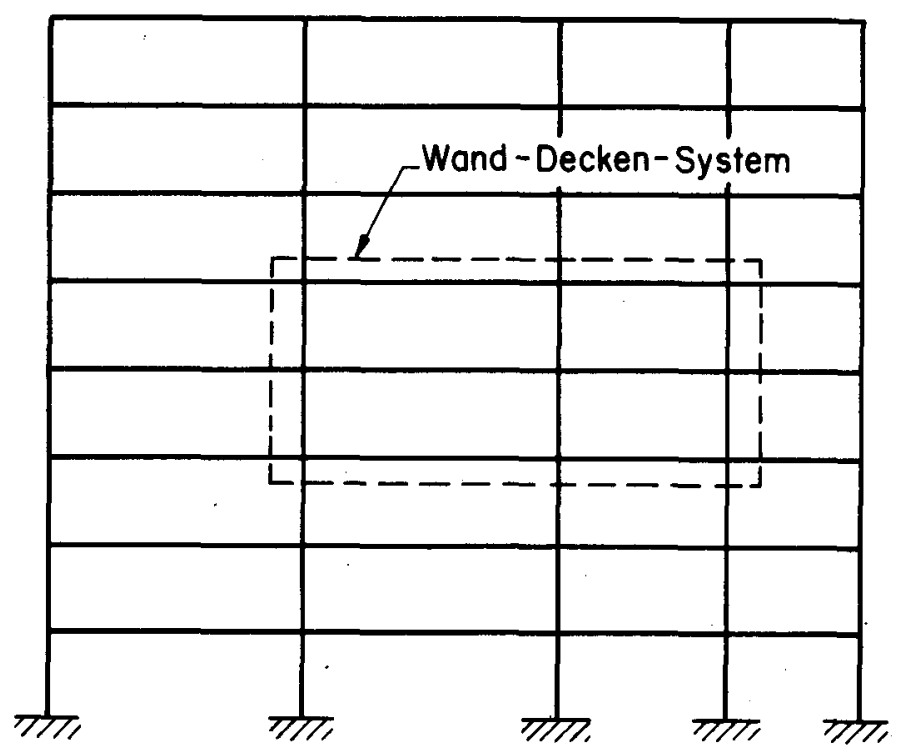

\section{Bild 7.1 : Stockwerkrahmen}

Grössere Stockwerkrahmensysteme (Bild 7.1) können mit linear elastischen Rechenmaschinen (u.a. Computerprogramme) behandelt werden. Eine genauere Analyse von Stockwerkrahmensystemen hat die fehlende Zugfestigkeit in den Verbindungen Wand - Decke sowie das nichtlineare Materialverhalten in Rechnung zu stellen. Die Berechnung grosser Stockwerkrahmensysteme ist bei einer derartigen Betrachtung mit einem erheblichen Rechenaufwand verbunden. Im Folgenden soll daher nebst der genaueren Untersuchung über das Zusammenwirken von Wänden und Decken auch gezeigt werden, wie aus dem Stockwerkrahmensystem kleinere Subsysteme herausgeschnitten und abgegrenzt werden können. Vor allem im Hinblick auf die Ermittlung der Wandbeanspruchungen und auf die Wandbemessung erscheinen einfache Methoden zur Behandlung von Rahmensystemen nützlich. 

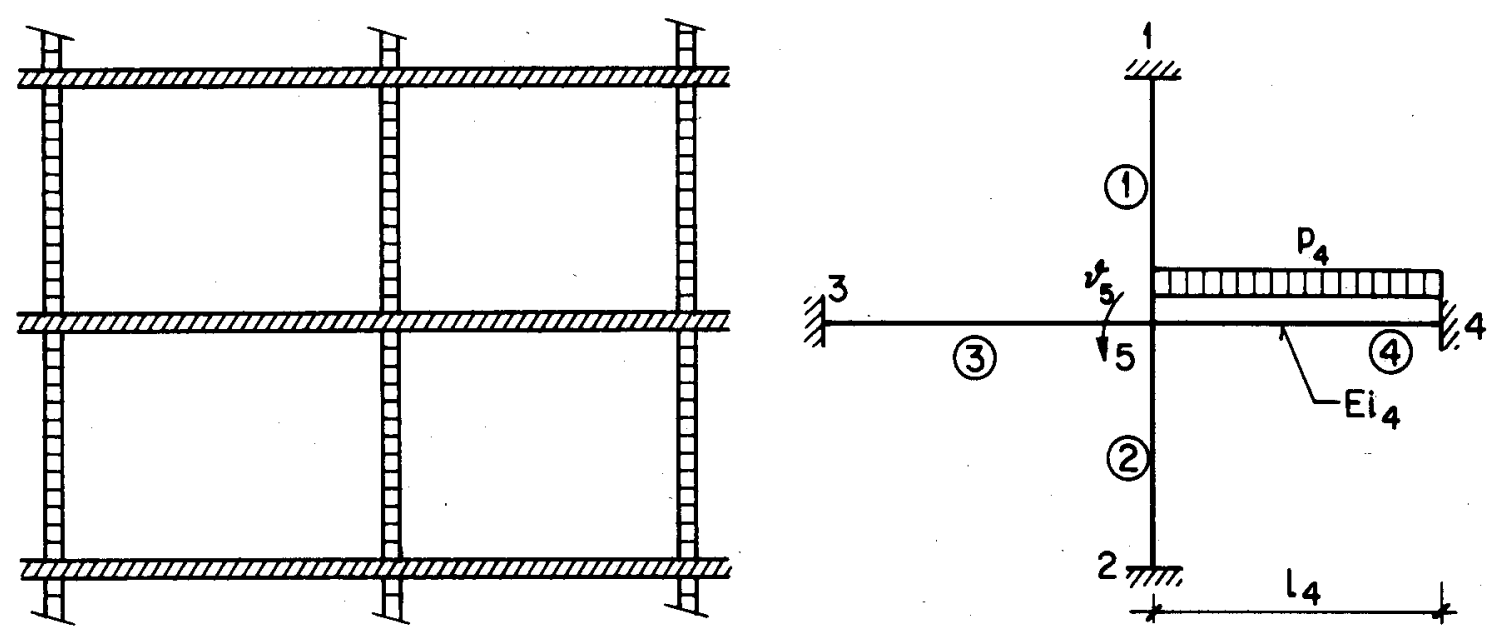

\section{Bild 7.2: Wand-Decken-System}

In Bild 7.2 ist der im Bild 7.1 angedeutete Ausschnitt eines Rahmensystems dargestellt. Es handelt sich um ein Wand-Decken-System. Unter der Annahme eines linear elastischen Materials sowie konstanter Biegesteifigkeiten der Stäbe 1 bis 4 lauten die Stabendmomente im Punkt 5 wie folgt:

$M_{5 i}=4 \cdot \frac{E I j}{I_{j}} \cdot v_{5}+M_{54}^{O}$

$M_{54}^{0}$ stellt das Festeinspannmoment dar. Für den gleichmässig mit $p_{4}$ belasteten, beidseits eingespannten Stab der Länge $I_{4}$ beträgt $M_{54}^{0}=P_{4} \cdot 1_{4}^{2} / 12$. Sofern im Knoten kein äusseres Moment angreift, lautet die Momentgleichgewichts-Bedingung im Knoten:

$\sum_{i=1}^{4} M_{5 i}=0$

Aus Gl. (7.2) und unter Verwendung von Gl. (7.1) kann der resultierende Knotendrehwinkel bestimmt werden:

$v_{5}=-\frac{M_{54}^{0}}{4 \cdot \sum_{j=1}^{4} \frac{E_{j}}{I_{j}}}$

Aus obiger Beziehung ist ersichtlich, dass die Knotenverdrehung proportional zur Summe der Festeinspannmomente und umgekehrt proportional zur Summe der auf die Stablängen bezogenen Biegesteifigkeiten der beteiligten Stäbe ist.

Eine solche Berechnung führt in gewissen Fällen zu unzutreffenden Resultaten. Insbesondere kann sich je nach Grösse der Axiallast in der Wand zwischen Deckenauflager und Wandende eine Differenzverdrehung ergeben. Das Zusammenwirken von Wänden und den darin eingespannten Decken soll daher unter Be- 
rücksichtigung der fehlenden Zugfestigkeit in den Verbindungen zwischen Wand und Decke sowie des nichtlinearen Materialverhaltens betrachtet werden.

In Bild 7.3 ist ein Deckenauflager dargestellt. Die Decke ist in die beiden Wandabschnitte 1 und 2 voll eingebunden und das rechte Deckenauflager voll eingespannt. Die Axiallast im oberen und unteren Wandabschnitt betrage vorerst $P_{1}$ und liege, da die Querkraft in der Decke gleich null ist, zentrisch. In Bild 7.3a sind die auf die Decke wirkenden Kräfte eingetragen.

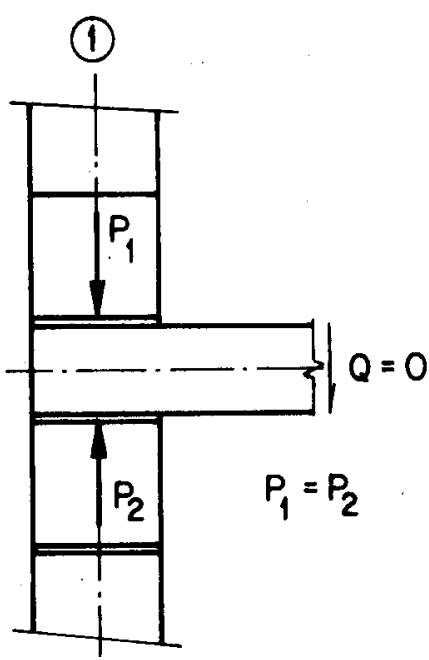

(2)

a)

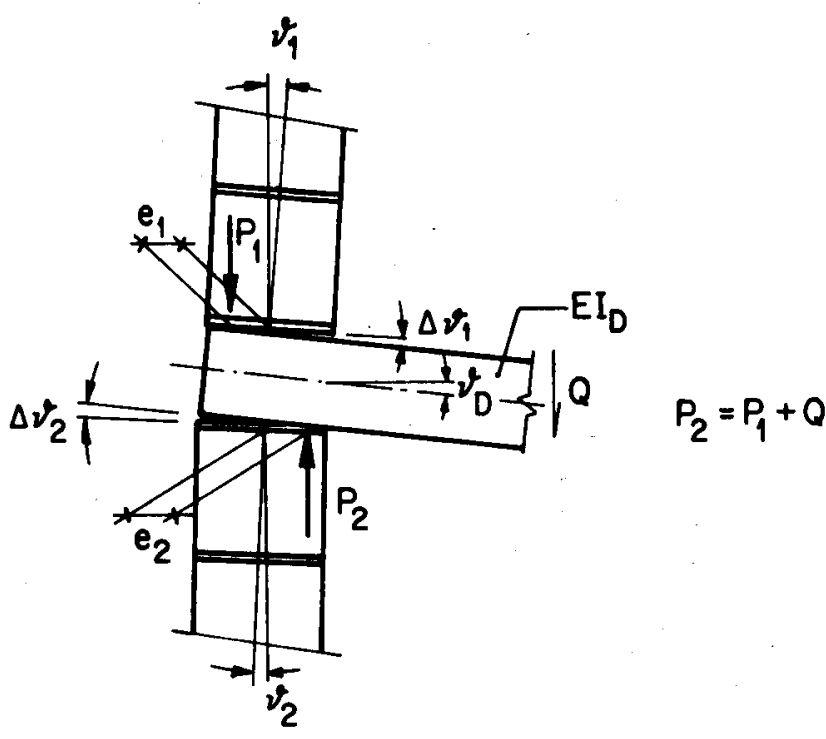

b)

\section{Bild 7.3 : Deckenauflager}

Wird nun die Decke belastet, so ergibt sich eine Verdrehung des Deckenauflagers (Bild 7.3b). Die Verdrehung führt einerseits zu einer exzentrischen Lage der Axiallasten, wobei im unteren Wandabschnitt letztere um die Querkraft aus der Decke zugenommen hat. Infolge der Exzentrizitäten $\varepsilon_{1}$ und $e_{2}$ ergeben sich die Wandendverdrehungen $\vartheta_{1}$ und $\vartheta_{2}$. Diese weichen um den Fugendrehwinkel $\Delta \vartheta_{1}$ bzw. $\Delta \vartheta_{2}$ vom Auflagerdrehwinkel $\vartheta_{D}$ der Decke ab. Andererseits wird die Decke durch die Wandabschnitte 1 und 2 eingespannt. Dieses Wandeinspannmoment Wergibt sich durch die exzentrische Lage von $P_{1}$ und $P_{2}$ und beträgt bezüglich der Wirkungsinie von $P_{2}$ :

$W\left(v_{D}\right)=\left\{e_{1}\left(v_{D}\right)+e_{2}\left(v_{D}\right)\right\} \cdot P_{1}$

Die Deckenspannweite 1 vermindert sich dabei um $e_{2}$. Die Exzentrizitäten $\varepsilon_{1}$ und $e_{2}$ sind vom Auflagerdrehwinkel $\vartheta_{D}$ der Decke abhängig (vgl. Kap. 6).

Für das System in Bild 7.3 ergibt sich damit und unter Berücksichtigung von G1. (7.1) und G1. (7.2) folgende Beziehung:

$4 \cdot \frac{E I_{D}}{I} \cdot \vartheta_{D}+W\left(v_{D}\right)-M_{D}^{O}=0$ 
G1. (7.5) kann anders geschrieben werden:

$W\left(\vartheta_{D}\right)=M_{D}^{O}-4 \cdot \frac{E I_{D}}{1} \cdot \vartheta_{D}=M_{D}\left(\vartheta_{D}\right)$

Der Ausdruck auf der rechten Seite der Gleichung stellt die lineare Beziehung zwischen dem Einspannmoment $M_{D}$ und dem Auflagerdrehwinkel $\vartheta_{D}$ der Decke auf der Grundlage eines linear elastischen Materials dar (Bild 7.4). Bei verschwindender Verdrehung entspricht $M_{D}$ dem Festeinspannmoment $M_{D}^{O}$. Bei der beidseitig voll eingespannten Decke unter gleichmässiger Belastung $p$ beträgt es $p \cdot l^{2} / 12$. An der Nullstelle ist die Verdrehung gleich dem Auflagerdrehwinkel $\vartheta_{D}^{\prime}$ der einseitig gelenkig gelagerten Decke. Er beträgt im vorliegenden Fall $\vartheta_{D}^{\prime}=p \cdot I^{3} /(48 \cdot E I)$.

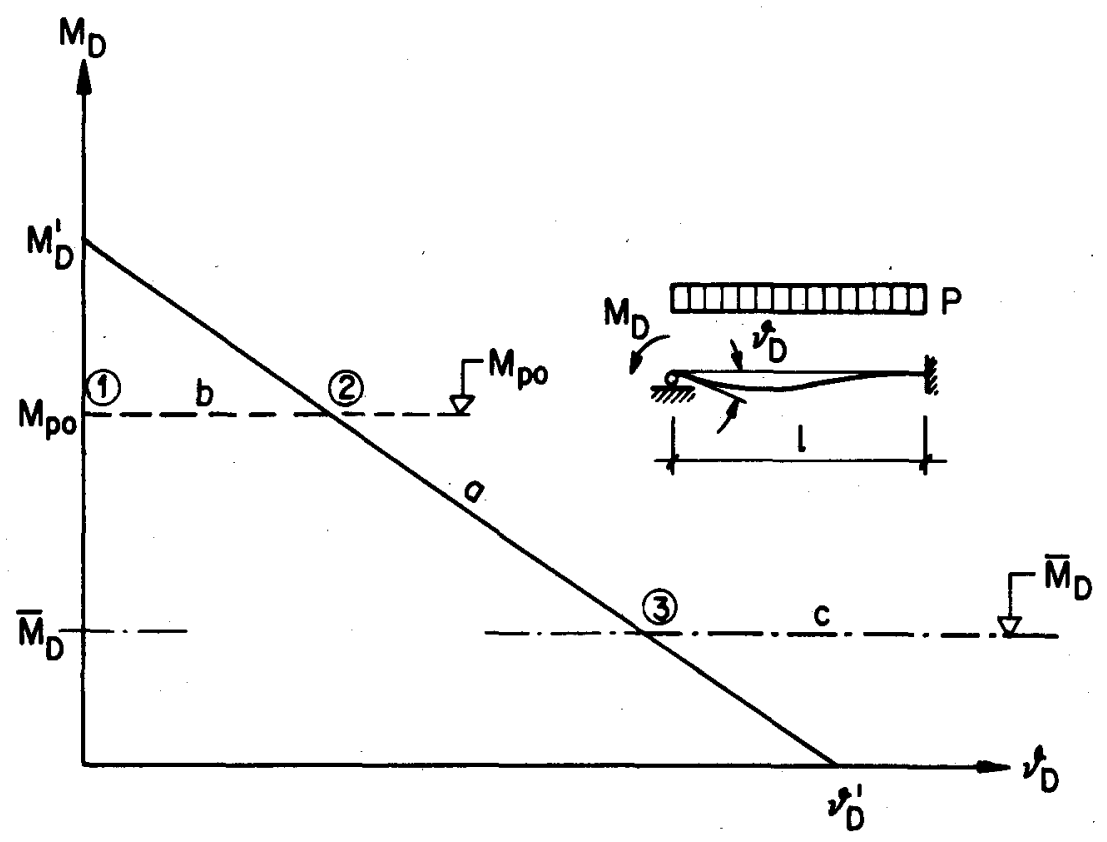

\section{Bild 7.4: Deckeneinspannmoment $M_{D}\left(v_{D}\right)$}

Jeder Punkt auf der Geraden a stellt für eine gegebene Belastung $p$ eine mögliche Gleichgewichtslage der Decke dar. Das hierzu notwendige Einspannmoment $M_{D}$ und die sich daraus ergebende Verdrehung sind also linear miteinander verknüpft. Setzt man für die Decke eine linear elastisch-ideal plastische Moment-Krümmungs-Beziehung voraus, so sind für die Beziehung zwischen Einspannmoment $M_{D}$ und dem Auflagerdrehwinkel der Decke zwei weitere Fälle zu beachten:

- Ist nämlich der plastische Biegewiderstand $M_{\text {po }}$ der Decke im Einspannquerschnitt kleiner als das bei der vorhandenen Auflagerverdrehung für das Gleichgewicht notwendige Einspannmoment, so ist der Gültigkeitsbereich der Geraden a entsprechend einzuschränken. Entlang der horizontal verlaufenden Geraden $b$ ist das Einspannmoment $M_{D}$ gleich dem plastischen Biegewiderstand $M_{\text {po }}$. Für jede Kombination von $M_{D}$ und $\vartheta_{D}$ auf dem Abschnitt 1-2 sind dann entsprechend dem durch $M_{\text {po }}$ beschränkten Einspannmoment die Feldbiegemomente in der Decke grösser. Bei grösseren Auflagerdrehwinkeln $\vartheta_{D}$ ist zur Er- 
füllung der Momentengleichgewichts-Bedingung der Decke nur noch ein Einspannmoment erforderlich, das kleiner ist als der plastische Biegewiderstand des Einspannquerschnittes.

- Es besteht nun aber auch die Möglichkeit, dass der plastische Biegewiderstand im meistbeanspruchten Feldquerschnitt kleiner ist als das Biegemoment, das sich in diesem Querschnitt aus einer linear elastischen Momentenverteilung (Kurve a) ergibt. Um die Decke mit den äusseren Lasten $p$ im Gleichgewicht zu halten, ist ein Resteinspannmoment $\bar{M}_{D}$ erforderlich. Der Gültigkeitsbereich der Geraden a kann demnach nicht nur nach oben, sondern auch nach unten beschränkt sein. Im Punkt 3 schliesst sich somit ein horizontal verlaufender Kurvenast $c$ an. Die rechte Seite der Beziehung (7.6) zerfällt damit in drei Funktionen mit verschiedenen Gültigkeitsbereichen:

$\begin{array}{ll}\text { a: } W\left(\vartheta_{D}\right)=M_{D}^{D}-4 \cdot \frac{E I}{1} \cdot \vartheta_{D} & : \vartheta_{D 2} \leq \vartheta_{D} \leq \vartheta_{D 3} \\ \text { b: } W\left(\vartheta_{D}\right)=M_{P D} & : 0 \leq \vartheta_{D} \leq \vartheta_{D 2} \\ \text { c: } W\left(\vartheta_{D}\right)=\bar{M}_{D} & : \vartheta_{D 3} \leq \vartheta_{D}\end{array}$

Im Verlauf der Funktion $W=W(\vartheta)$ sind nun ebenfalls einige Besonderheiten kurz darzulegen. W ist das Produkt der Summe der Exzentrizitäten $e_{1}$ und $e_{2}$ in den beiden Wandabschnitten und der Axiallast $P_{1}$ im oberen Wandabschnitt (vgl. Gl. (7.4)). Bei konstanter Axiallast $P_{1}$ wachsen die Exzentrizitäten $e_{1}$ und $e_{2}$ infolge des nichtlinearen Materialverhaltens und des Einflusses 2. Ordnung unterproportional zur Verdrehung an. Das Maximum von $W$ ist durch die unter der vorhandenen Axiallasten $P_{1}$ und $P_{2}$ maximalen Exzentrizitäten bestimmt. Bei verschwindenden Axiallasten sind die maximalen Exzentrizitäten gleich der halben Querschnittbreite.

In Bild 7.5 sind für drei verschiedene Lasten $P_{1}$ die $W-\vartheta_{D}$-Kurven aufgetragen. Bei kleiner Last $P_{i}^{\prime}$ (Kurve d) ergibt sich bereits bei einem geringen $W$-Wert eine deutliche Abflachung der Kurve. Bei weiterer Verdrehung verläuft sie dann praktisch horizontal, da in diesem Bereich die Exzentrizitäten $e_{1}$ und $\theta_{2}$ nur noch wenig anwachsen. Der Wandbruch stellt sich bei kleinen Lasten unter grossen Verdrehungen ein. Er ist durch ein Kreuz gekennzeichnet. Ist nun P" grösser, so verläuft die Kurve e anfänglich gleich wie die Kurve d (bei Vernachlässigung der 2. Ordnung), die Abflachung setzt jedoch erst bei einem grösseren $W$ ein. Die Kurve überschreitet bei grösserer Verdrehung ein Maximum, und der Wandbruch stellt sich im Bereich des abfallenden Kurvenastes ein. Bei grosser Axiallast $P_{1}^{\prime \prime}$ erfolgt der Wandbruch bereits bei kleinen Verdrehungen, d.h. im ansteigenden Abschnitt der Kurve f. Die Neigung dieser Kurve ist infolge des verstärkten Einfluss 2. Ordnung kleiner als diejenige der Kurven $d$ und e. Schlussendich sei noch ein besonderer Fall erwähnt (Kurve g). Bei grossen Schlankheiten der Wände und geringen Axiallasten $P_{1}$ und $P_{2}$ ergibt sich bei der kritischen Verdrehung $\vartheta_{0, k r}$ ein Stabilitätsver- 


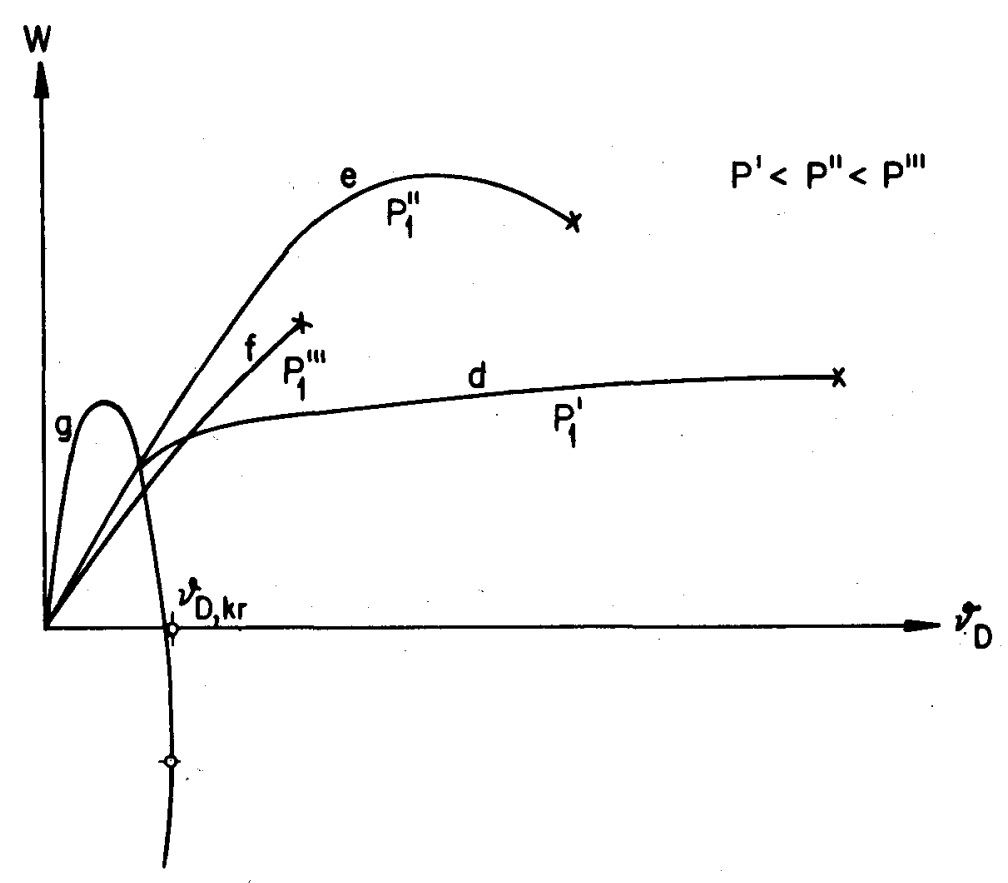

Bild 7.5: Wandeinspannmoment, $W\left(\mathcal{\nu}_{D}\right)$

sagen. Die Wände verlieren ihr Tragvermögen, ohne dass in einem Querschnitt ein Materialversagen aufgetreten ist (vgl. Abschn. 6.2.2.1, 6.3.4).

Mit Hilfe der Darstellungen in den Bildern 7.4 und 7.5 kann nun für die Gleichungen (7.7) graphisch eine Lösung bestimmt werden. Unter dem gesuchten Auflagerdrehwinkel der Decke $\vartheta_{D}$ ist die Gl. (7.7) erfüllt.

Zunächst werde eine Decke betrachtet, deren Charakteristik der Kurve a in Bild 7.4 entspricht. Sie ist in Bild 7.6 a eingezeichnet. Die resultierende Verdrehung ist durch den Schnittpunkt dieser Kurve mit den $W-\vartheta_{D}$-Kurven bestimmt. Trägt man nun auf der Ordinate die Axiallast $P_{1}$ und auf der Abszisse die zugehörige resultierende Verdrehung auf, erhält man die in Bild 7.6 dargestellte Beziehung. Ohne Auflast entspricht $\vartheta_{D}^{\prime}$ dem Auflagerdrehwinkel der gelenkig gelagerten Decke. Mit zunehmender Axiallast nimmt die resultierende Verdrehung markant ab. In diesem Abschnitt sind die Exzentrizitäten der Axiallasten beinahe gleich der halben Querschnittsbreite. Bei grösseren Axiallasten ist die resultierende Verdrehung durch die Steifigkeitsverhältnisse von Decke und Wänden gegeben. Infolge des Einflusses 2. Ordnung nimmt die Verdrehung bei grösseren Axiallasten wieder zu.

Beim linear elastischen Material ergäbe sich unter Vernachlässigung der 2. Ordnung eine ordinatenparallele Gerade. Die resultierende Verdrehung ist in diesem Fall nur durch die Steifigkeitsverhältnisse gegeben und unabhängig von der Axiallast. Diese Gerade ist in Bild 7.6 strichpunktiert eingetragen.

Die sich ergebende Axiallastunabhängigkeit der resultierenden Verdrehung $\vartheta_{D}$ entspricht jedoch in vielen Fällen nicht den tatsächlichen Verhältnissen in einem Bauwerk. Anhand eines einfachen Beispiels soll dies gezeigt werden. 


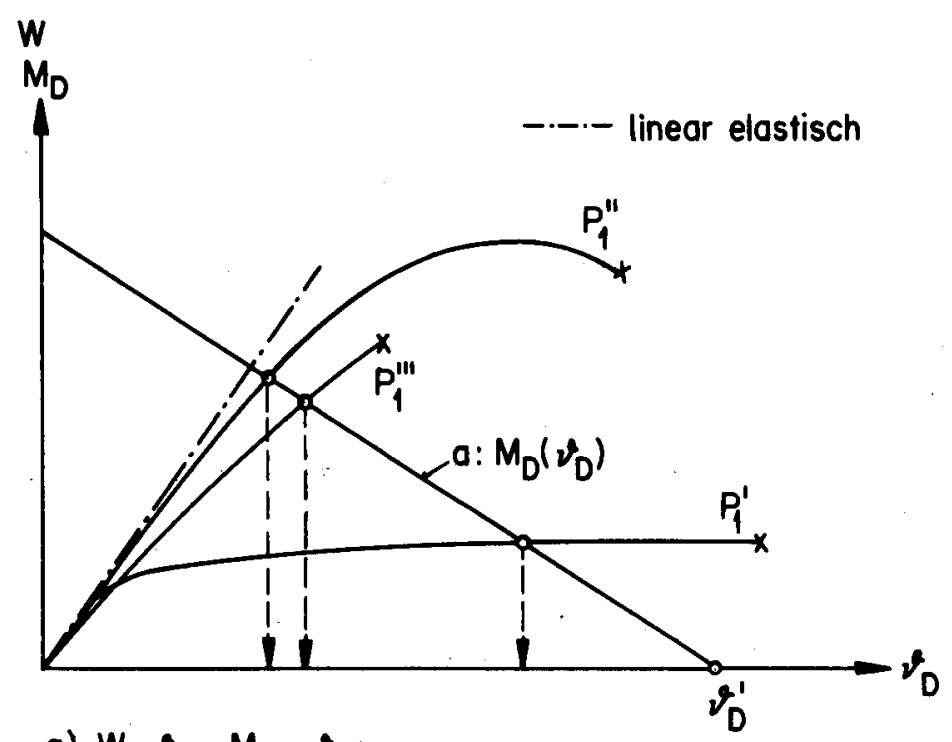

a) $W \cdot v_{D}, M_{D}-v_{D}$

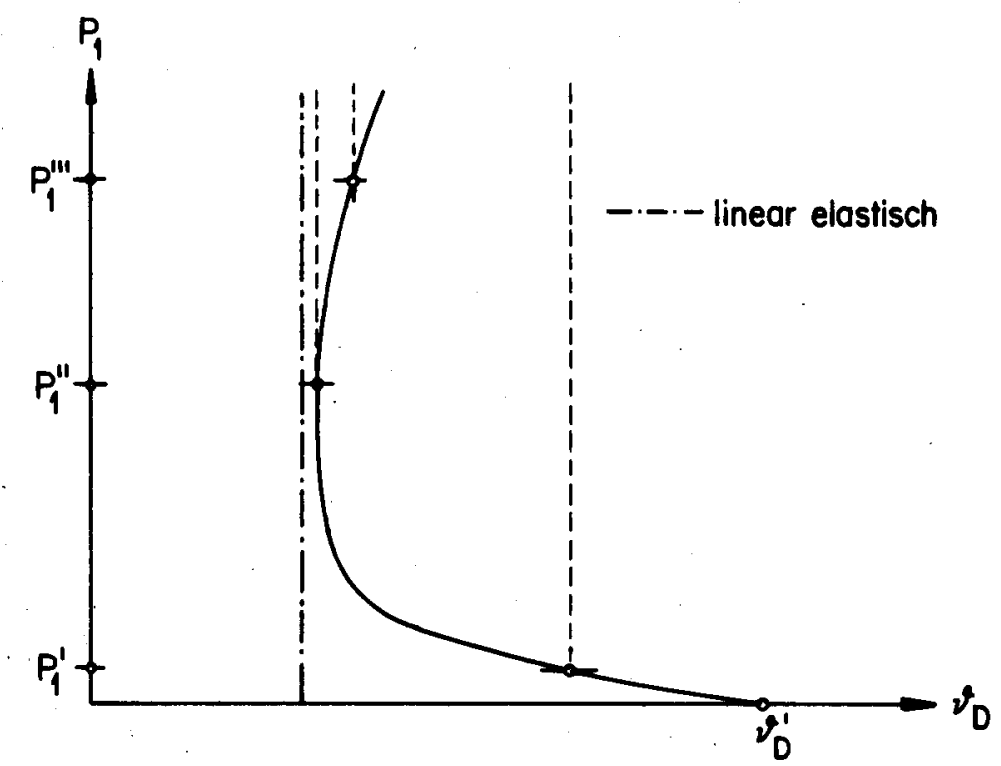

\section{Bild 7.6: Axiallast-resultierender Auflagerdrehwinkel $\nu_{D}$}

Der in Bild 7.7 dargestellte Stockwerkrahmen weist übliche Abmessungen auf. Die gleichmässig verteilte Nutzlast der Decke beträgt $2 \mathrm{kN} / \mathrm{m}$ ' und die ständige Last $g$ beträgt $4 \mathrm{kN} / \mathrm{m}^{\prime}$.

Die aus einer elastischen Rahmenrechnung resultierenden Momenten-und Normalkraftverteilungen infolge von Eigengewicht und Nutzlast sind nebenstehend dargestellt. Die Normalkraft entspricht dabei der Axiallast P. Die Exzentrizität der Axiallast in den Wänden ergibt sich durch den Quotienten von Biegemoment und Axiallast:

$e=\frac{M}{P}$ 


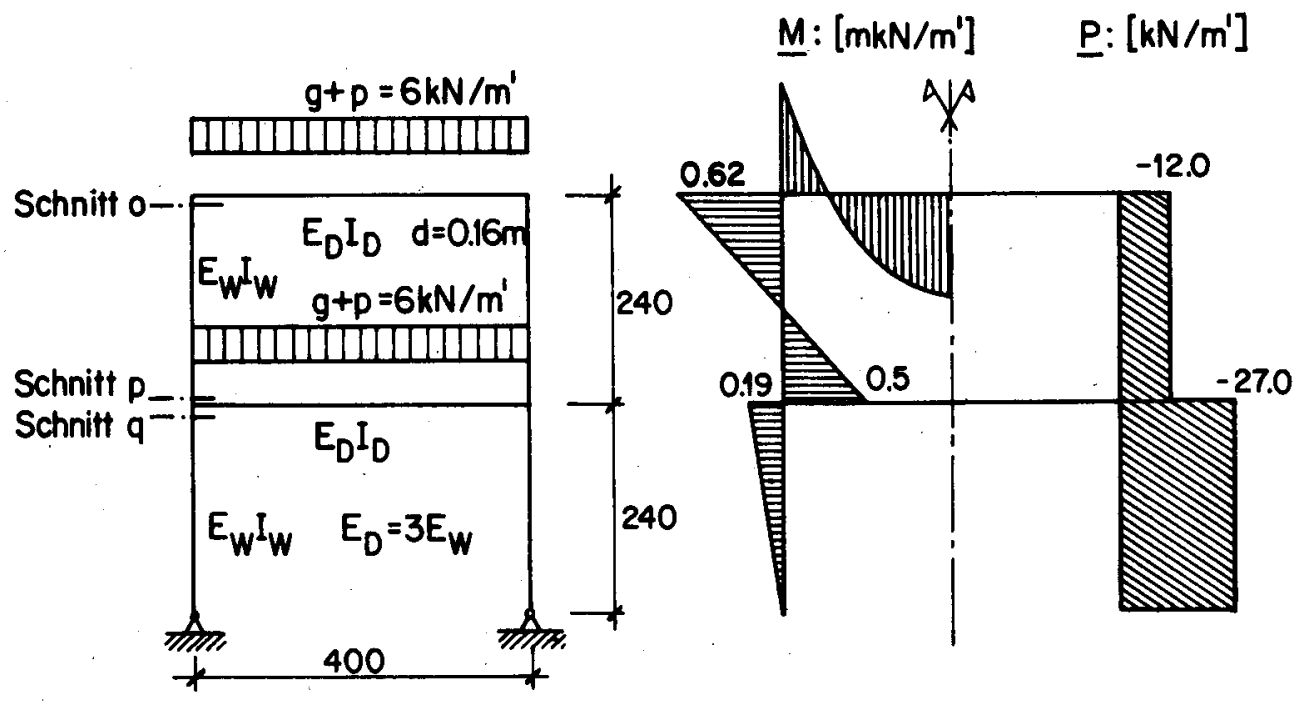

Bild 7.7 : Elastische Rahmenberechnung

Demnach betragen die Exzentrizitäten in den Schnitten $0, p$ und $q: 51,41$ und $7 \mathrm{~cm}$. Die beiden ersten Werte sind offensichtlich unzutreffend. Da die Verbindung Wand - Decke keine Zugkräfte aufnehmen kann, muss die Wirkungslinie der Axiallast aus Gleichgewichtsgründen innerhalb des Wandquerschnittes liegen; die Exzentrizität kann daher die halbe Querschnittsbreite nicht übersteigen. Umgekehrt wird die Auflagerverdrehung $\vartheta_{D}$ der Decken bei der linear elastischen Berechnung unterschätzt, da die Deckeneinspannung überschätzt wird.

In Bild 7.8 sind nun noch einige spezielle Fälle im Zusammenwirken von Wänden und Decken dargestellt. Die Kurven in Bild 7.8 a haben im wesentlichen den gleichen Verlauf wie in Bild 7.6 a, sie schneiden sich aber nicht. Dies bedeutet, dass sich zwischen Wänden und Decke kein Gleichgewichtszustand einstellen kann. Das Wandeinspannmoment $W\left(\vartheta_{D}\right)$ ist im Vergleich zum Einspannmoment $M_{D}$, das für eine Gleichgewichtslage der Decke erforderlich wäre, zu gering. In diesem Fall müssten entweder die Deckenspannweite bzw. die Auflast verringert oder die Steifigkeit der Decke bzw. der Wände erhöht werden.

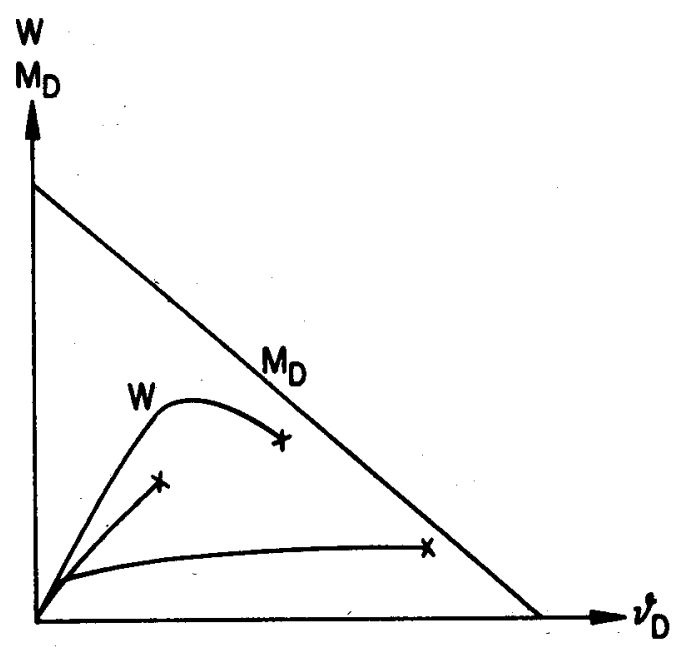

a)

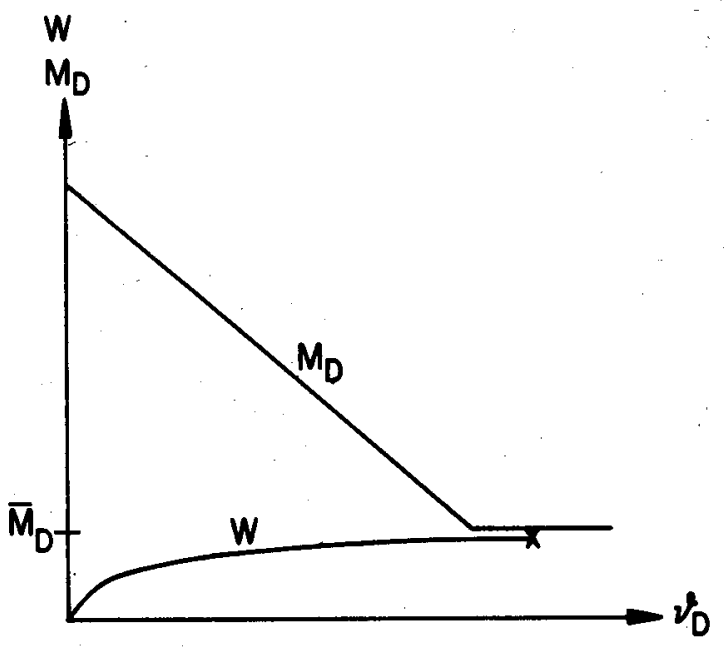

b)

Bild 7.8 : Spezialfälle 
Ein ähnlicher Fall ergibt sich in Bild 7.8 b. Der plastische Biegewiderstand der Decke im Feldbereich ist zu gering und muss erhöht werden. Um das vorliegende Wand-Decken-System im Gleichgewicht halten zu können, wäre ein Deckeneinspannmoment $\bar{M}_{D}$ erforderlich. Bei einem grösseren plastischen Feldbiegewiderstand verringert sich $\bar{M}_{D}$.

\subsection{Subsysteme}

In Bild 7.9 ist ein 18-stöckiges Rahmensystem dargestellt. Die Stockwerkhöhe beträgt $2.40 \mathrm{~m}$ und die durchlaufenden Stahlbetondecken weisen Spannweiten von 5 und $3.5 \mathrm{~m}$ sowie eine Stärke von $18 \mathrm{~cm}$ auf. Die gleichmässig verteilte Nutzlast $p$ beträgt $2 \mathrm{kN} / \mathrm{m}^{\prime}$ und die ständige Last g $4.5 \mathrm{kN} / \mathrm{m}^{\prime}$.

Bei der Berechnung eines Rahmensystems stehen grundsätzlich zwei Methoden zur Verfügung. Man berechnet die Beanspruchungen am globalen System simultan oder unterteilt es in einzelne subsysteme und berücksichtigt die Lagerungsbedingungen von Wänden und Decke an den Schnittstellen.

Im Folgenden soll letztere Methode am Beispiel des Rahmensystems von Bild 7.9 dargestellt werden. Für die Ermittlung der Beanspruchungen der Aussenwände ist es zweckmässig, das Subsystem gemäss Bild 7.10 abzugrenzen. Da die Axiallast in der Mauerwerkswand für das Zusammenwirken von Wand und Decke von grosser Bedeutung ist (Bild 7.6) werden drei Subsysteme mit geringer ( $P=$ $50 \mathrm{kN} / \mathrm{m}$ ', mittlerer (200) und grosser (300) Axiallast in der Wand behandelt.

$N=P \cdot$ (Wände links)
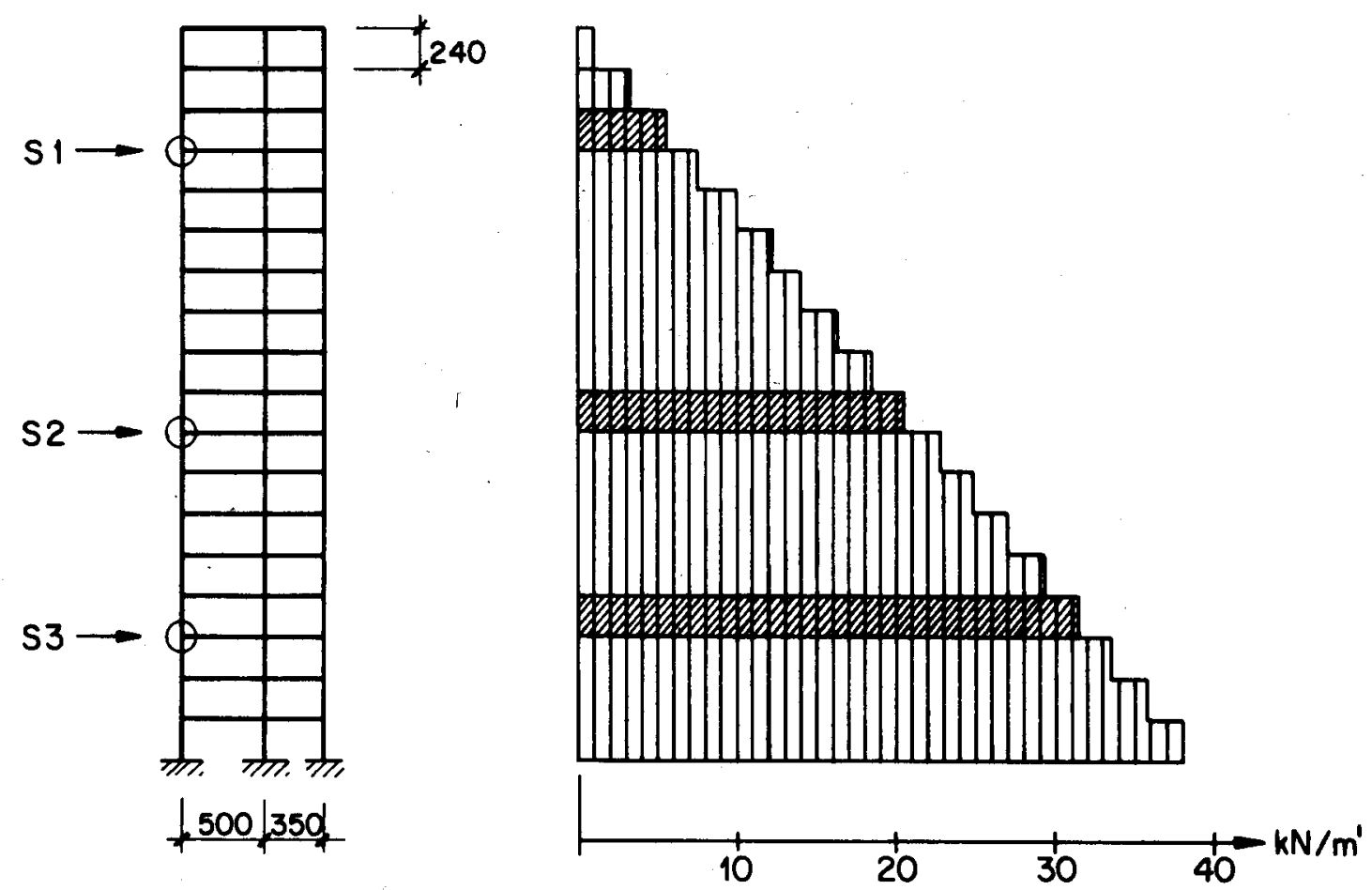

Bild 7.9: Rahmensystem 
Die Lage der Subsysteme ist in Bild 7.9 mit einem Kreis gekennzeichnet. Die Grösse der Axiallasten $P$ kann aus dem nebenstehenden Normalkraft-Diagramm bestimmt werden.

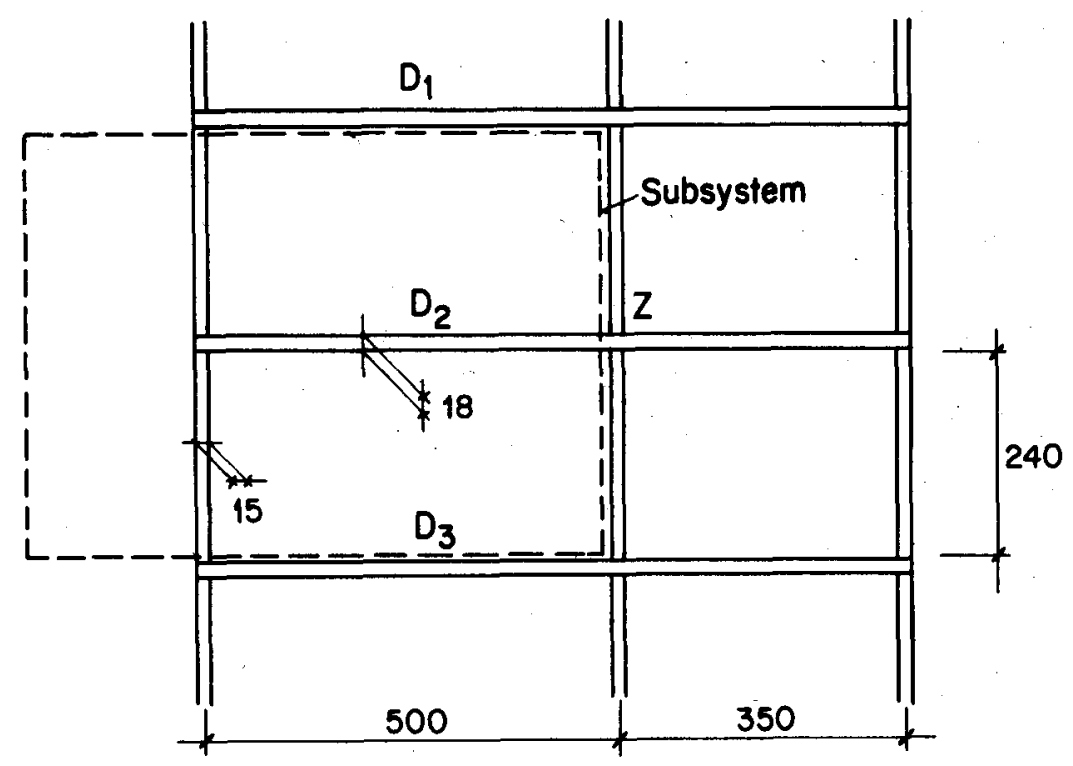

\section{Bild 7.10: Abgrenzung des Subsystems}

Für jedes dieser drei Subsysteme werden die effektiven Lagerungsbedingungen durch die folgenden extremen Lagerungsbedingungen eingegabelt (Bild 7.11):

Wände: I Die unten und oben angrenzenden Decken D1 und D3 sind starr.

Sie verdrehen sich nicht, d.h. $\vartheta_{D 1}=\vartheta_{D 3}=0$.

II Die angrenzenden Deckenauflager verdrehen sich um den gleichen Betrag wie dasjenige von Decke $\mathrm{D} 2$ im Subsystem: $\vartheta_{01}=\vartheta_{\mathrm{D} 2}=\vartheta_{\mathrm{D} 3}$.

Decke: III Die Decke ist beim inneren Knoten $Z$ voll eingespannt.

IV Das Einspannmoment der Decke beim Knoten $Z$ beträgt: $(p+g) \cdot 1^{2} / 24$.

$V \quad$ Die Decke ist bei $Z$ gelenkig gelagert.

Wände

Decke
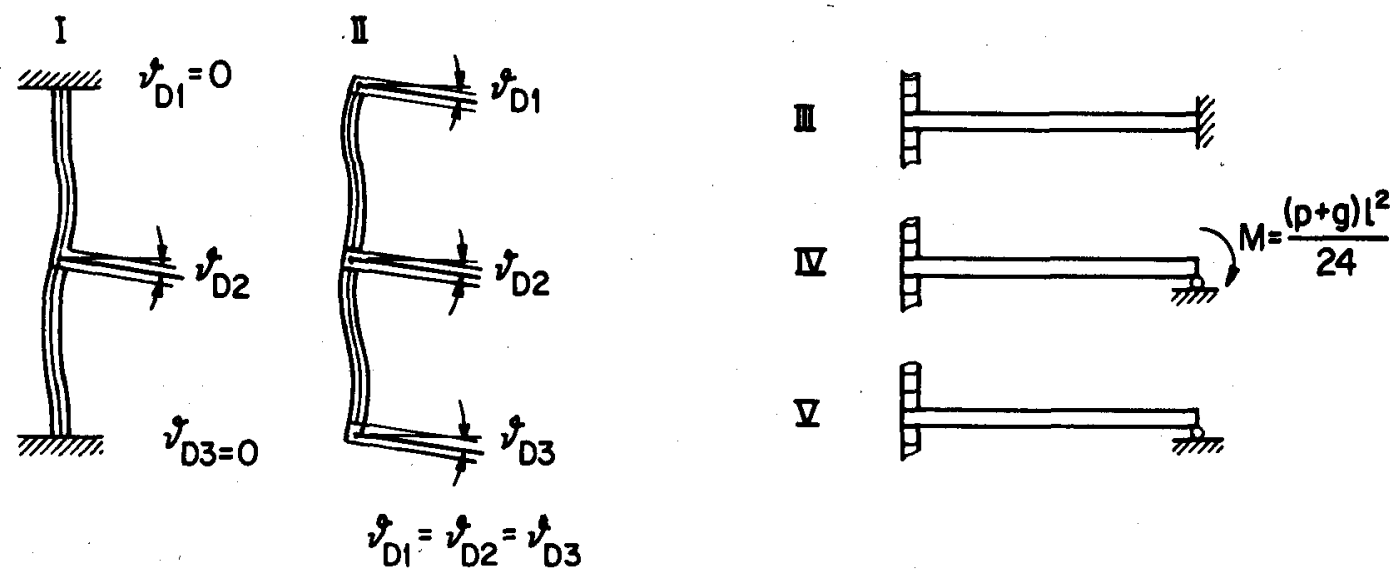

Bild 7.11 : Extreme Lagerungsbedingungen für Wände und Decken 
Mit dem Verfahren von Abschnitt 7.2 lässt sich nun der resultierende Auflagerdrehwinkel $\vartheta_{D 2}$ im Subsystem bestimmen. Aufgrund der verschiedenen angenommenen extremen Lagerungsbedingungen der Wände und der Decke erhält man verschiedene Ergebnisse. Aus den Unterschieden können die maximalen Abweichungen, die sich durch die Abgrenzung der Subsysteme gegenüber dem Rahmensystem ergeben können, abgeschätzt werden.

Für die rechnerische Ermittlung der Deckencharakteristik $M_{D}=M_{D}\left(\vartheta_{D}\right)$ wurde ein linear elastisches Materialverhalten zugrunde gelegt. Mit den Annahmen III, IV und $V$ erhält man die drei in Bild 7.12 dargestellten Geraden.

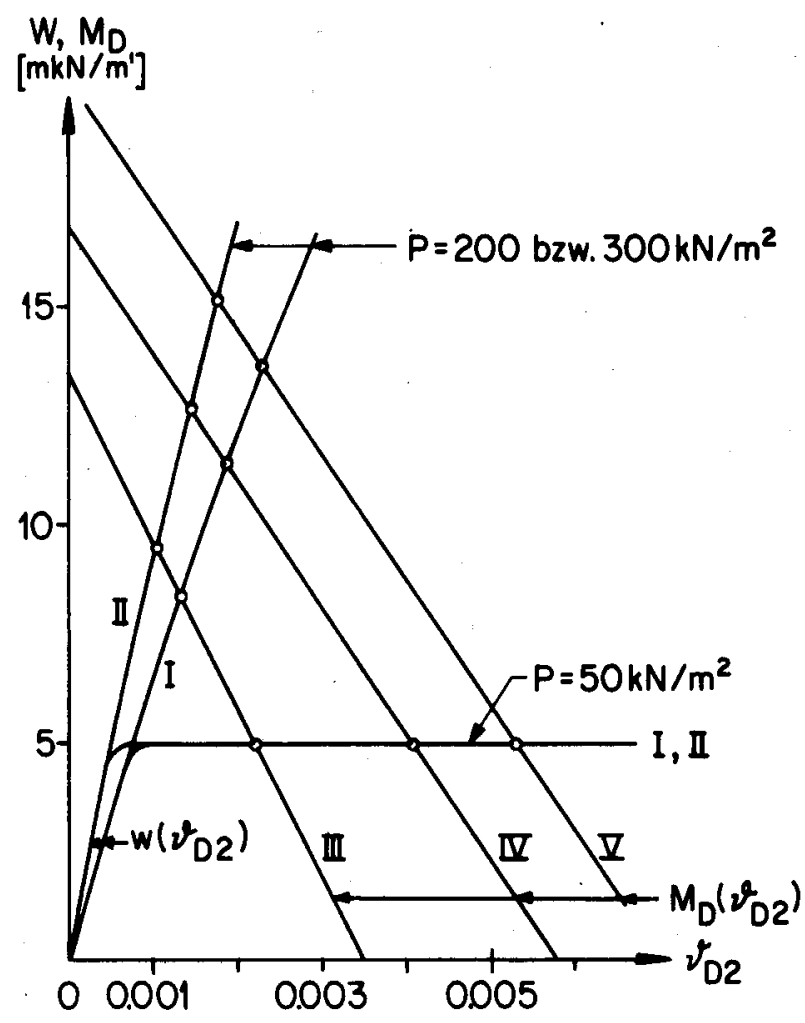

Bild 7.12: Resultierender Auflagerdrehwinkel $\vartheta_{\text {D2 }}$

Der Verlauf von $W$ als Funktion von $\vartheta_{02}$ (Bild 7.12) wurde mit dem StarrkörperFeder-Modell bzw. mit dem darauf beruhenden Computerprogramm für das $15 \mathrm{~cm}$ MBHC berechnet. Die Drehfedercharakteristiken (vgl. Abschn. 6.4.1) basieren auf dem Stoffgesetz von Bild 5.5. Es zeigt sich, dass die Lagerbedingung (I und II) keinen grossen Einfluss auf den Verlauf der $W-\vartheta$ D2 -Kurven hat.

In Bild 7.13 sind die resultierenden Auflagerdrehwinkel der Decke tabellarisch zusammengestellt:

- Die Lagerbedingung der Wände (I, II) hat keinen grossen Einfluss auf die Grösse von $\vartheta_{\mathrm{D} 2}$. Die Werte weichen je nach Einspannverhältnissen der Decke in $Z$ bis $z u 26 \%$ voneinander ab.

- Die Lagerungsbedingung bzw. die Einspannverhältnisse der Decke beim inneren Knoten $Z$ haben auf die Grösse von $\vartheta_{02}$ einen grossen Einfluss. Für die Lagerungsbedingungen III, IV und $V$ ergeben sich Abweichungen bis zu $60 \%$. 


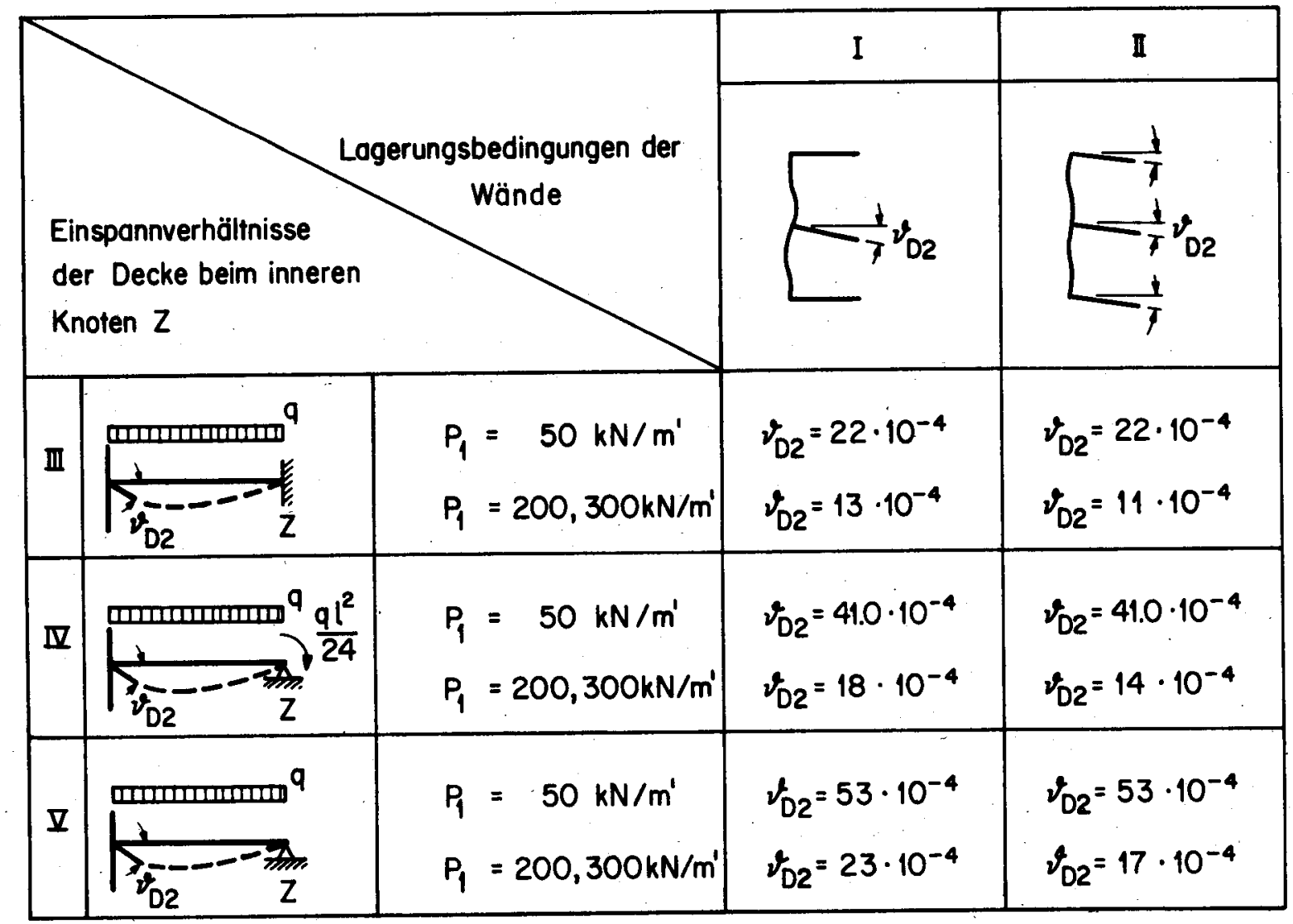

Bild 7.13: Resultierender Auflagerdrehwinkel $\vartheta_{\mathrm{D} 2}$

Bei der Beurteilung dieser Abweichungen ist jedoch zu berücksichtigen, dass die Lağerungsbedingungen aus den statischen Verhältnissen näherungsweise abgeschätzt werden können. 


\section{Bemessung von Mauerwerkswänden}

\subsection{Ueberblick}

Den Ausgangspunkt jeder Bemessung bilden die Anforderungen, die an ein Bauwerk gestellt werden. Zum einen wird erwartet, dass die vorgesehene Nutzung des Bauwerks gewährleistet werden kann, und zum andern soll die Sicherheit aller im Einflussbereich des Bauwerks befindlichen Menschen garantiert sein.

Den herkömmlichen Bemessungsverfahren im Mauerwerksbau liegt wohl der Gedanke zugrunde, durch die Beschränkung der maximalen rechnerischen Spannungen auf nominelle, zulässige Spannungen beide Anforderungen, also die Gebrauchsfähigkeit und die Bruchsicherheit, gleichzeitig zu gewährleisten. Zahlreiche Schadenfälle an Mauerwerksbauten weisen jedoch darauf hin, dass insbesondere die Gebrauchsfähigkeit mit derartigen Bemessungsverfahren nicht immer in genügendem Masse sichergestellt werden konnte.

Im Stahl- und Stahlbetonbau beginnen sich in letzter Zeit neue Bemessungsverfahren durchzusetzen. Sie halten die Begriffe Gebrauchsfähigkeit und Tragfähigkeit klar auseinander und verlangen eine getrennte Bauwerksbemessung auf diese beiden Grenzzustände. In Bild 8.1 ist dieses Bemessungskonzept schematisch dargestellt.

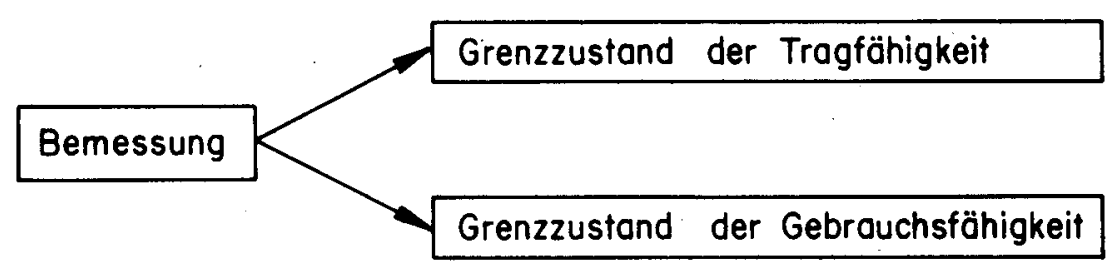

\section{Bild 8.1: Bemessungskonzept}

Mit den experimentellen und den vorliegenden theoretischen Untersuchungen wurden die Grundlagen zur Ausarbeitung eines solchen neuartigen Bemessungsverfahrens für Mauerwerkswände (Backstein und Kalksandstein) unter Axiallast und Biegung geschaffen. Das Bemessungsverfahren ist im Anhang A5 dargestellt und soll im Folgenden erläutert werden. 


\section{2 Bemessungskonzept}

Wie einleitend erwähnt und in Bild 8.1 dargestellt, beruht dieses Bemessungsverfahren auf der gesonderten Bemessung auf den Grenzzustand der Tragfähigkeit (Bruchsicherheit) und auf den Grenzzustand der Gebrauchsfähigkeit.

Für die Bemessung sind die in 6.1 dargesteliten Beanspruchungsarten $E$ und $V$ von Bedeutung. Für jede Beanspruchungsart wurden im vorliegenden Bemessungsverfahren je drei Bemessungsfälle festgelegt (Bild 8.2).

Beanspruchungsart $E$ (vorgegebene Exzentrizität)

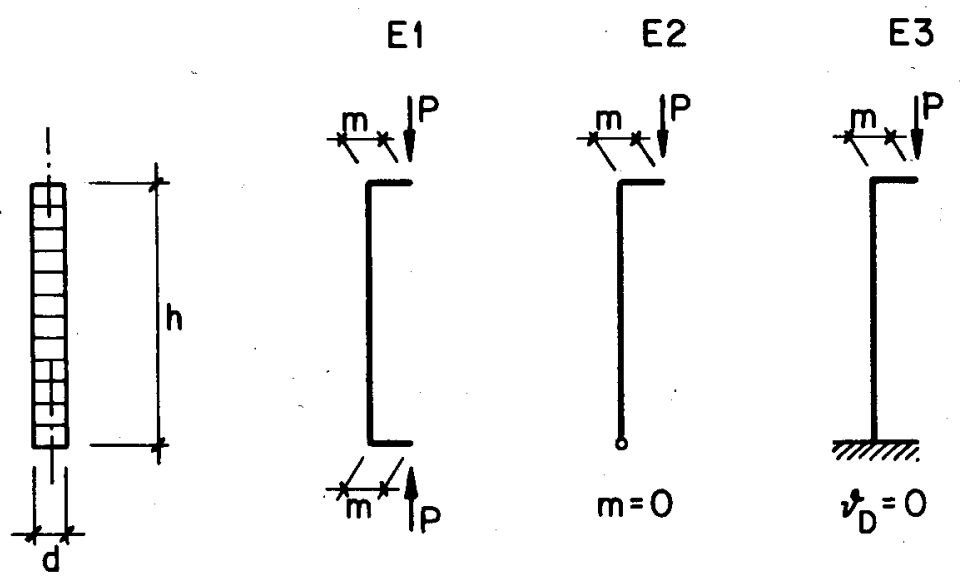

Beanspruchungsart $V$ (Verdrehung der Deckenauflager)

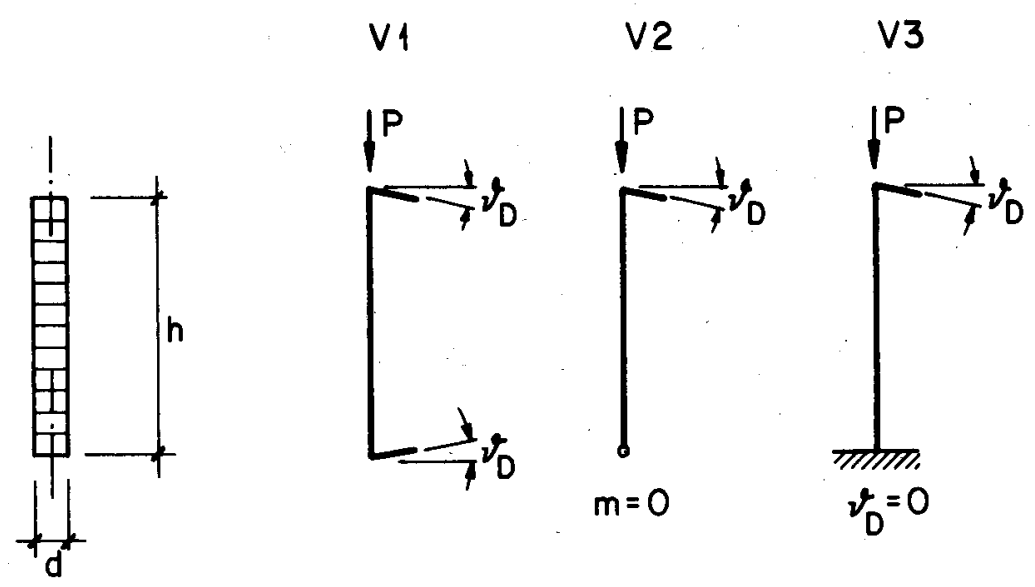

Bild 8.2: Bemessungsfälle 
Für die Bemessung ist defjenige Fall zu bestimmen, welcher der tatsächlichen Wandbeanspruchung am ehesten entspricht. In den Fällen E1 und V1 ist die Wand symmetrisch beansprucht. Bei den Fällen E2 und V2 wird vorausgesetzt, dass ein Wandende frei drehbar und zentrisch gelagert ist, und in E3 bzw. V3 wird angenommen, dass das eine Wandende auf einer starren Decke oder Fundamentplatte liegt. In allen angegebenen Fällen sind die Wände horizontal gehalten. Das Exzentrizitätsmass m entspricht der auf die Kernweite des Rechteckquerschnittes bezogenen Exzentrizität. Die Wandschlankheit $\delta$ ist entsprechend den bisherigen Untersuchungen das Verhältn்is zwischen Wandhöhe h und Wanddicke d.

Bei allen verwendeten Spannungen ( $\sigma_{B}, \sigma_{\Gamma b}$, $\sigma_{\Gamma g}$ ) handelt es sich um Bruttospannungen. Da die Lochflächenanteile der Mauersteine sehr unterschiedlich sind, wurde im Bemessungsverfahren eine Möglichkeit zu deren Berücksichtigung angegeben.

\subsection{Nachweis der Bruchsicherheit}

Der Nachweis der Bruchsicherheit beruht auf der Forderung, dass die um einen Materialfaktor $f_{m}$ reduzierte Traglast $P^{*}$ die Beanspruchung infolge der mit einem Lastfaktor $f_{I}$ multiplizierten Belastung $P$ übersteigt. Analytisch ergibt sich folgende Beziehung:

$\frac{P^{*}}{f_{m}} \geq P \cdot f_{1}$

Wird $P^{*}$ bzw. P auf die Bruttoquerschnittsfläche der Wand bezogen, so lautet Gl. (8.1) wie folgt:

$\frac{\sigma^{*}}{f_{m}}=\sigma_{B} \geq \sigma_{r b}$

$\sigma_{B}$ wird als Bemessungsfestigkeit und $\sigma_{r b}$ als rechnerische Spannung bezeichnet.

Traglastspannung $\sigma^{*}$ - Bemessungsfestigkeit $\sigma_{B}$

Im Fall der Beanspruchungsart $E$ hängt $\sigma^{*}$ vom Exzentrizitätsmass $m$ und der Wandschlankheit $\delta$ ab (Bild 8.3 a). Bei der Beanspruchungsart $V$ ist $\sigma^{*}$ durch die Auflagerverdrehung $\vartheta_{0}$ der Decke und wiederum $\delta$ bestimmt (Bild 8.3 b). In Kapitel 6 wurden diese Zusammenhänge theoretisch untersucht.

Mit der Reduktion von $\sigma^{*}$ und $\sigma_{B}$ um den Materialfaktor $f_{m}(G 1$. (8.2)) werden Unsicherheiten bezüglich der Streuungen in den Materialeigenschaften, des Rechenmodells zur Bestimmung von $\sigma^{*}$, der Verarbeitungsqualität bei der Erstellung u.a. abgedeckt. Die Art der Reduktion von $\sigma^{*}$ auf $\sigma_{B}$ ist in Bild 8.4 dargestelit. Der Materialfaktor beträgt 3.0 . 


\section{a) Beanspruchungsart $E$}

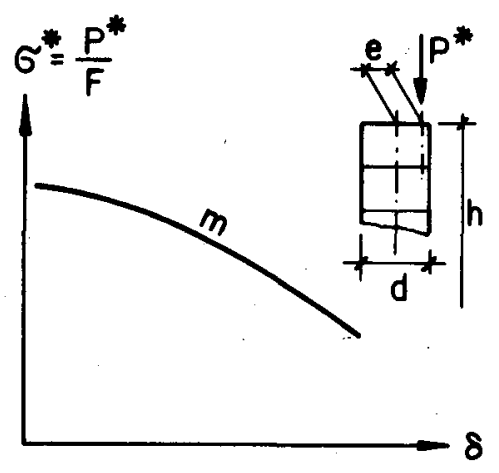

Bild 8.3 : Traglastspannung $\sigma^{*}$ b) Beanspruchungsart $V$

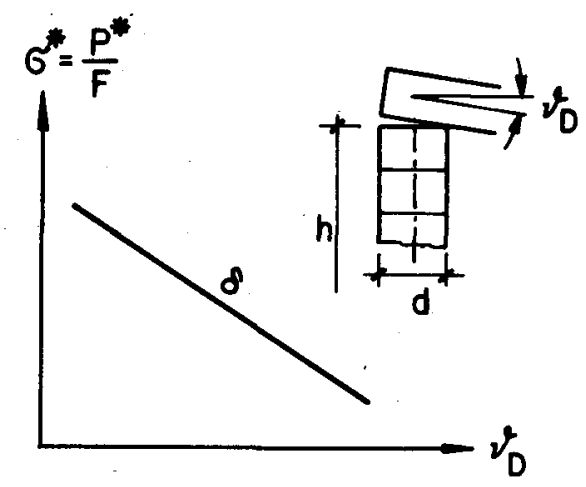

\section{a) Beanspruchungsart $E$}

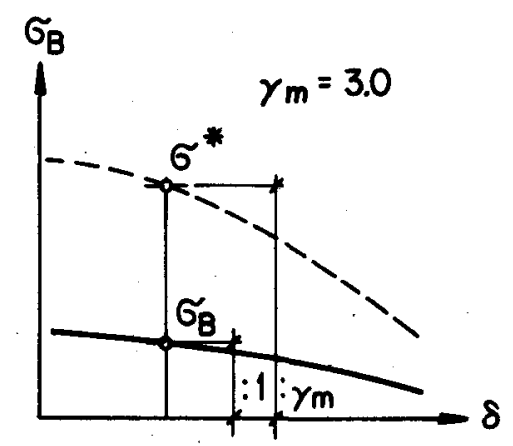

b) Beanspruchungsart $V$

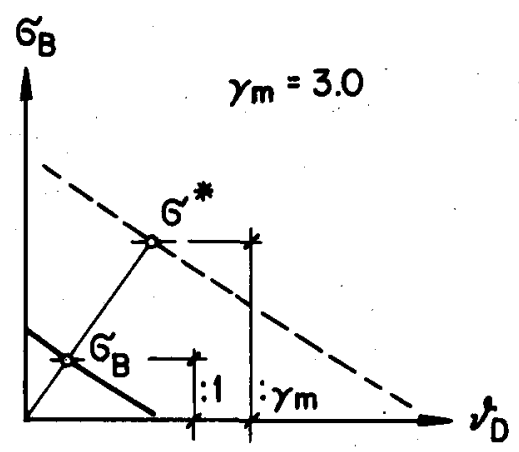

\section{Bild 8.4: Reduktion von $\sigma^{*}$ auf $\sigma_{B}$}

Die in Bild 8.4 angegebenen Kurven für $\sigma_{B}$ wurden im Bemessungsverfahren für die Fälle E1 bis E3 bzw. V1 bis V3 mit dem Starrkörper-Feder-Modell (vgl. Abschn. 6.4) berechnet und als Bemessungsdiagramme BE bzW. BV bezeichnet. $B$ bezieht sich auf den Nachweis der Bruchsicherheit und $E, V$ auf die Beanspruchungsart.

Die Berechnung dieser Bemessungsdiagramme für Backsteinmauerwerk beruhte auf dem $15 \mathrm{~cm}$ MBHC und dem Stoffgesetz von Bild 5.5. Der Lochflächenanteil der Mauersteine betrug 50\%. Für andere Mauerwerksqualitäten (MBNC, MBSC, ...) können diese Diagramme umgerechnet werden.

\section{Rechnerische Spannung o rb}

Für die Bestimmung von $\sigma_{\Gamma b}$ sind im Bemessungsverfahren Lastkombinationsgesetze angegeben. Sie wurden aus der RL 34 der Norm SIA 162 übernommen. Die einzelnen Lastgrössen werden mit teilweise unterschiedlichen Faktoren gewichtet. Währenddem die einzelnen Lastannahmen deterministische Grössen sind, weisen die einzelnen Gewichte, die Lastfaktoren $f_{1}$, probabilistische Züge 
auf.

Lochflächenanteile der Mauersteine, die von $50 \%$ abweichen, können durch Umrechnung von $\sigma_{\text {rb }}$ berücksichtigt werden.

\subsection{Nachweis der Gebrauchsfähigkeit}

Der Grenzzustand der Gebrauchsfähigkeit lässt sich nicht durch ein einziges, eindeutiges Kriterium festlegen. Im Bemessungsverfahren wird davon ausgegangen, dass die Gebrauchsfähigkeit dann gewährleistet ist, wenn unter den massgebenden Lasten, Verdrehungen der Deckenauflager sowie Zwängungen infolge von Kriech-, Schwind- und Temperaturverformungen der Decken keine Risse auftreten bzw. nur solche, die keine Folgeschäden nach sich ziehen und das Aussehen der Wand nicht beeinträchtigen.

Unter Axiallast und Biegung können sich solche Risse in den horizontal verlaufenden Lagerfugen sowie in der Verbindung Wand - Decke ergeben (vgl. Kapitel 5 und 6). Bei der Festlegung der angegebenen Richtwerte für die Rissweiten war in Betracht zu ziehen, dass die Art der Wandkonstruktion und die äusseren Einflüsse im Hinblick auf Folgeschäden von Bedeutung sind. Daneben spielt bei der Beurteilung der Rissbildung der Zeitpunkt des Auftragens des Verputzes eine Rolle. Wird der Verputz erst einige Zeit nach Fertigstellung des Rohbaus aufgetragen, kann angenommen werden, dass der grösste Teil der Schwind- und Kriechverformungen bereits stattgefunden hat und damit die Rissanfälligkeit reduziert worden ist.

Selbstverständlich bewirken Rissbildungen bei verkleideten Mauerwerkswänden (Vorhangfassade, Aussenisolation u.a.) in der Regel keine Folgeschäden und beeinträchtigen das Aussehen der Wand nicht. Für solche Mauerwerkswände kann ein Nachweis der Gebrauchsfähigkeit entfallen.

Mit dem Bemessungsverfahren können mit Hilfe der Bemessungsdiagramme GV 1 bis GV3 (für die Fälle V1 bis V3) die grössten rechnerisch zu erwartenden Rissweiten in den Lagerfugen bzw. der Verbindung Wand - Decke bestimmt werden. $G$ bezieht sich auf den Nachweis der Gebrauchsfähigkeit und $V$ auf die Beanspruchungsart. Die Berechnung dieser Diagramme beruhte ebenfalls auf dem $15 \mathrm{~cm}$ MBHC und dem Stoffgesetz von Bild 5.5. In Abschnitt 6.4.2 wurde gezeigt, dass die Uebereinstimmung zwischen rechnerisch und experimentell ermittelten Werten befriedigend ist (Bilder 6.41 und 6.42).

Bei bekannter Wandschlankheit $\delta$, Verdrehung $\vartheta_{D}$ des Deckenauflagers sowie rechnerischer Spannung $\sigma_{r g}$ kann die grösste rechnerisch zu erwartende Rissweite aus den Diagrammen GV1 bis GV3 gemäss Bild 8.5 bestimmt werden. 


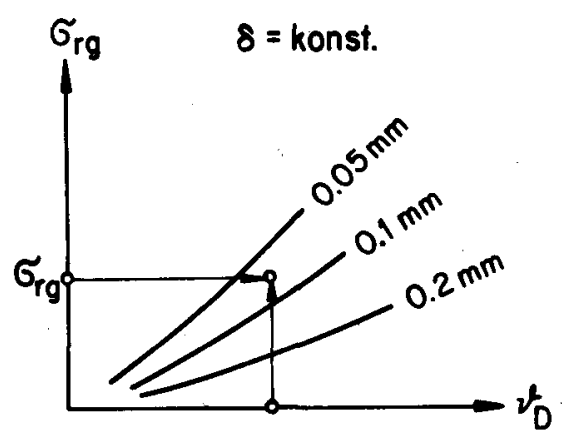

\section{Bild 8.5 : Bestimmung der maximalen Rissweite}

$\sigma_{r g}$ ergibt sich aus ständigen Lasten und solchen Nutz- und Verkehrslasten, die für die Rissbildung massgebend sind.

Im Fall der Beanspruchungsart E (Fälle E1 bis E3) können diese Rissweitẹn ebenfalls bestimmt werden. Aus den Diagrammen DE1 bis DE3 (vgl. Abschnitt 8.6) kann bei gegebener Schlankheit $\delta, \sigma_{r g}$ und $m$ die sich ergebende Verdrehung des Deckenauflagers bestimmt werden (Bild 8.6).

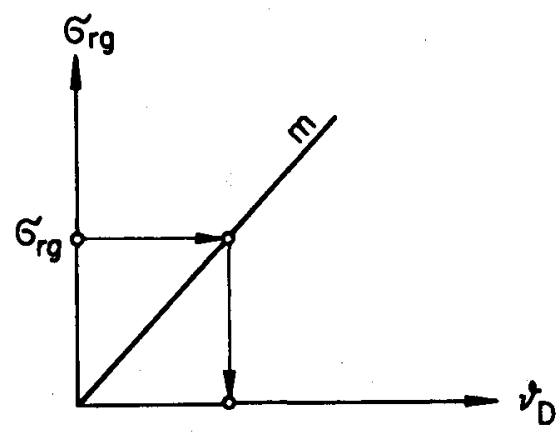

\section{Bild 8.6: Ermittlung von $\vartheta_{0}$}

Mit diesem Wert $\vartheta_{0}$ kann hernach entsprechend Bild 8.5 die Rissweite ermittelt werden.

\subsection{Berücksichtigung von Kriech-, Schwind- und Temperaturverformungen}

Die Kriechverformungen der Decke spielen für das Zusammenwirken von Wänden und Decken und damit für die Bestimmung der resultierenden Auflagerverdrehung der Decke eine Rolle (vgl. 8.6).

Infolge von Schwind- und Temperaturverformungen können sich im Bauwerk horizontale Relativverschiebungen $\Delta l$ der Deckenauflager ergeben. Diese $\Delta l$ führen zu relativen Verdrehungen der Deckenauflager gegenüber der Wandaxe (Bild 8.7). Die sich daraus ergebenden Auflagerdrehwinkel $\vartheta_{D}$ der Decken müssen für den Nachweis der Gebrauchsfähigkeit mitberücksichtigt werden. Sie sind dement- 
$\underline{\Delta l}$

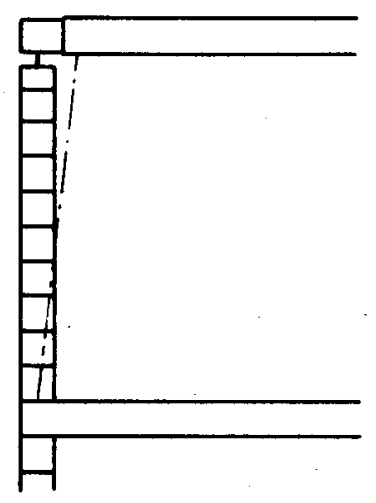

$\Delta \mathrm{l}$

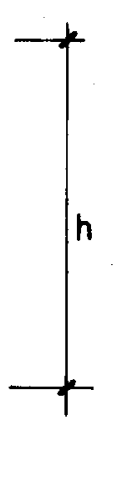

$$
v_{D}=\frac{\Delta l}{h}
$$

\section{Bild 8.7: Zwängung als Wandendverdrehung}

dementsprechend mit den Auflagerdrehwinkeln, die sich aus statischen Gründen ergeben (vgl. 8.6) zu überlagern. Die Wahl des Bemessungsfalls hat aufgrund dieses überlagerten $\vartheta_{0} z u$ erfolgen.

\subsection{Auflagerdrehwinkel $\vartheta_{D}$ der Decke}

Der im Bemessungsverfahren angegebene Weg zur Bestimmung von $\vartheta_{D}$ basiert auf den Untersuchungen über das Zusammenwirken von Wänden und Decken in Kapitel 7.

$M_{D}=M_{D}\left(\vartheta_{D}\right)$ entspricht dem Einspannmoment der Decke, das bei der Verdrehung $\vartheta_{0}$ für das Deckengleichgewicht notwendig ist. Bei linear elastischem und homogenem Material ergibt sich ein linearer Verlauf von $M_{D}\left(\vartheta_{D}\right)$.

$W=W\left(\vartheta_{D}\right)$ kann mit den Diagrammen DE (für den betreffenden Bemessungsfall) bestimmt werden. Bei bekannter rechnerischer spannung $\sigma_{\text {rb }} b z w \sigma_{r g}$ und Wandschlankheit $\delta$ kann $\vartheta_{D}$ für die m-Werte von 0.5, 1.0, 1.5 und 2.0 gemäss Bild 8.8 bestimmt werden. $W\left(\vartheta_{D}\right)$ wird gemäss Gl. (7.4) ermittelt.

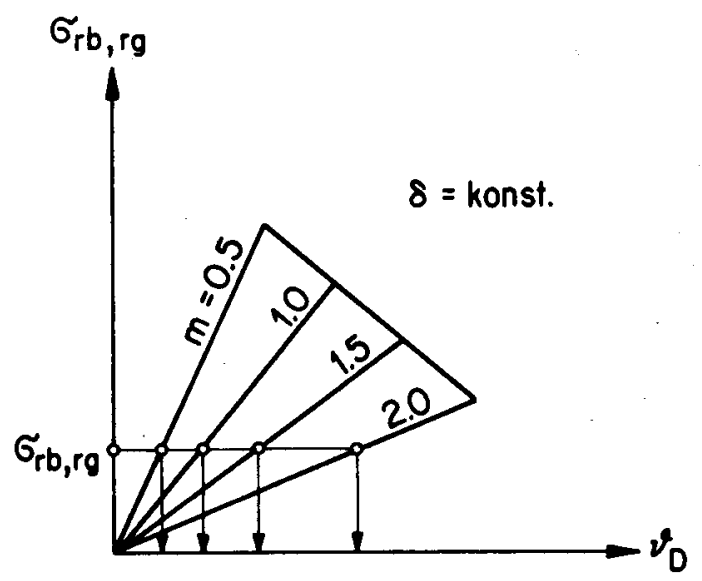

Bild 8.8: Bestimmung von $v_{D}$ aus $\sigma_{r b, r g}, m, \delta$ 
Der resultierende Auflagerdrehwinkel $\vartheta_{D}$ der Decke ergibt sich aus dem Schnittpunkt der Kurven $M_{D}=M_{D}\left(v_{D}\right)$ und $W=W\left(v_{D}\right)$. 


\section{Zusammenfassung}

Die Zielsetzung der vorliegenden Arbeit besteht in der theoretischen Untersuchung des Tragverhaltens von Mauerwerkswänden unter Druck und Biegung.

Die kurze Literaturübersicht in Kapitel 2 zeigt, dass systematische Untersuchungen über das Tragverhalten von Mauerwerkswänden erst in den letzten 30 bis 40 Jahren begonnen wurden.

In Kapitel 3 werden die hauptsächlichsten technisch-physikalischen Eigenschaften von Mauerwerk beschrieben. Sie ergeben sich aus den Eigenschaften und dem Zusammenwirken der beiden Komponenten Stein und Mörtel.

Auf der Grundlage von drei einfachen Stoffgesetzen und zwei extremen Annahmen über die Lochung werden im Kapitel 4 das Exzentrizitäts-Krümmungs-Verhalten $(e-\Phi)$ sowie die Interaktion M-P theoretisch untersucht. Die Zugfestigkeit ist für Rissbetrachtungen vor allem im Bereich kleiner Axiallasten von Bedeutung. Demgegenüber spielt sie für das Bruchverhalten praktisch keine Rol1e. Plastische Verformbarkeit des Materials führt zu grässeren Randstauchungen bis zum Bruch und dementsprechend zu grösseren Bruchkrümmungen. Die Untersuchungen über den Einfluss der Lochung zeigen, dass insbesondere der Lochflächenanteil für Riss- und Bruchbetrachtungen in Rechnung zu stellen ist. Demgegenüber ist der Einfluss der Lochanordnung (bei gleichem bzw. konstantem Lochflächenanteil) geringer.

In Kapitel 5 werden rechnerisch und experimentell ermittelte e- $\Phi-K u r v e n$ einander gegenübergestellt. Die Uebereinstimmung der Kurven ist gut. Die rechnerischen Kurven wurden auf der Grundlage eines nominellen Stoffgesetzes und des effektiven Querschnittes (Lochung) ermittelt.

Die Beziehungen zwischen der Axiallast $P$, den Exzentrizitäten e und Wandendverdrehungen $\vartheta$ sowie der Wandschlankheit $\delta$ werden in Kapitel 6 einerseits durch analytisch geschlossene Lösungen hergeleitet und andererseits mit Hilfe eines Starrkörper-Feder-Modells numerisch bestimmt. Analytisch geschlossene Ausdrücke als Lösungen der Differentialgleichung der ausgebogenen Wandaxe ergeben sich nur unter den vereinfachten Annahmen eines linear elastischen Materials mit und ohne Zugfestigkeit sowie eines Rechteckvollquerschnittes. Mit Hilfe dieser Beziehungen wird das grundsätzliche Tragverhalten einer symmetrisch gemäss Beanspruchungsart $E$ und $V$ belasteten Wand beschrieben. Das Starrkörper-Feder-Modell kann numerisch mit der Methode der Column Deflection Curves, deren Berechnung und Anwendung ausführlich dargestellt sind, behandelt werden. Die Mauersteine werden als Starrkörper idealisiert, und die Drehfedern als deren Verbindungselemente entsprechen den Fugen. Der Vergleich von rechnerischen und experimentellen Exzentrizitäts-Verdrehungs-Kurven zeigt eine gute Uebereinstimmung. Es darf daher angenommen werden, dass 
das Tragverhalten von Mauerwerkswänden mit dem Starrkärper-Feder-Modell rechnerisch beschrieben werden kann.

In Kapitel 7 wird das Zusammenwirken von Wänden und Decken untersucht. Grössere Rahmensysteme können in einzelne Subsysteme unterteilt werden, wobei die Lagerungsbedingungen von Wänden und Decken an den Schnittstellen berücksichtigt werden müssen. Am Beispiel eines 18-stöckigen Rahmensystems wird dieses Verfahren gezeigt.

Im Rahmen des Forschungsprojektes wurde ein neuartiges Bemessungsverfahren für Mauerwerkswände unter Axiallast und Biegung entwickelt. Entsprechend den neueren Entwicklungen im Stahl- und Stahlbetonbau verlangt es eine getrennte Bemessung auf den Grenzzustand der Tragfähigkeit (Bruchsicherheit) und den Grenzzustand der Gebrauchsfähigkeit. Das Bemessungsverfahren ist im Anhang A5 dargestellt und wird in Kapitel 8 erläutert. 


\section{Summary}

The objective of this work is the theoretical investigation of the load bearing behaviour of masonry walls subjected to axial load and bending moment.

The short survey of literature in the second chapter shows that the load bearing behaviour of such walls was not studied systematically before the last thirty to forty years.

In the third chapter, the main material properties of masonry are described. They depend on the material properties and on the interaction of the components respectively the bricks and the mortar.

Assuming three simple stress-strain-curves respectively two extreme cross sectional shapes of the perforation of the brick, the eccentricity-curvaturecurves as well as the interaction of the axial load and the bending moment are discussed in the fourth chapter. It is shown that cracking behaviour of masonry depends on its tensile strength. Plastic deformation of material leads to higher ultimate curvatures. For cracking as well as for rupture consideration the percentage portion of brick perforation is of much more importance than the cross sectional shape of the perforation of the brick.

In the fifth chapter, theoretical and experimental eccentricity-curvaturecurves are compared. The theoretical curves are calculated numerically and are based on a nominal stress-strain-curve and the effective cross sectional shape of brick perforation. Fair agreement is obtained.

Relations between the axial load, the eccentricity and the angle of rotation at the ends of the wall as well as the slenderness ratio are analytically respectively numerically obtained in the sixth chapter. The differential equation of the deflected wall axis can only be solved if a linear elastic material with or without tensile strength and a rectangular cross section is assumed. The analytical solution is used to discuss qualitatively the load bearing behaviour of a symmetrically loaded wall (loading case $E$ and $V$ ). For the numerical calculation a new type of model was used. The bricks are idealized as rigid elements and the mortar joints as springs, connecting the elements. Eccentricity-angle of rotation-curves are calculated with this model using the method of the column deflection curves. The comparison of these curves with the experimental curves shows fair agreement.

In the seventh chapter, the interaction of floors and masonry walls is discussed. It is shown that big frame systems can be subdivided into small subsystems.

A new design method for masonry walls under axial load and bending moment, 
represented in the appendix $A 5$, is explained in the eigth chapter. Two limit states, the serviceability limit state and the ultimate limit state, have to be considered. 


\section{Bezeichnungen}

\section{Geometrische Grössen}

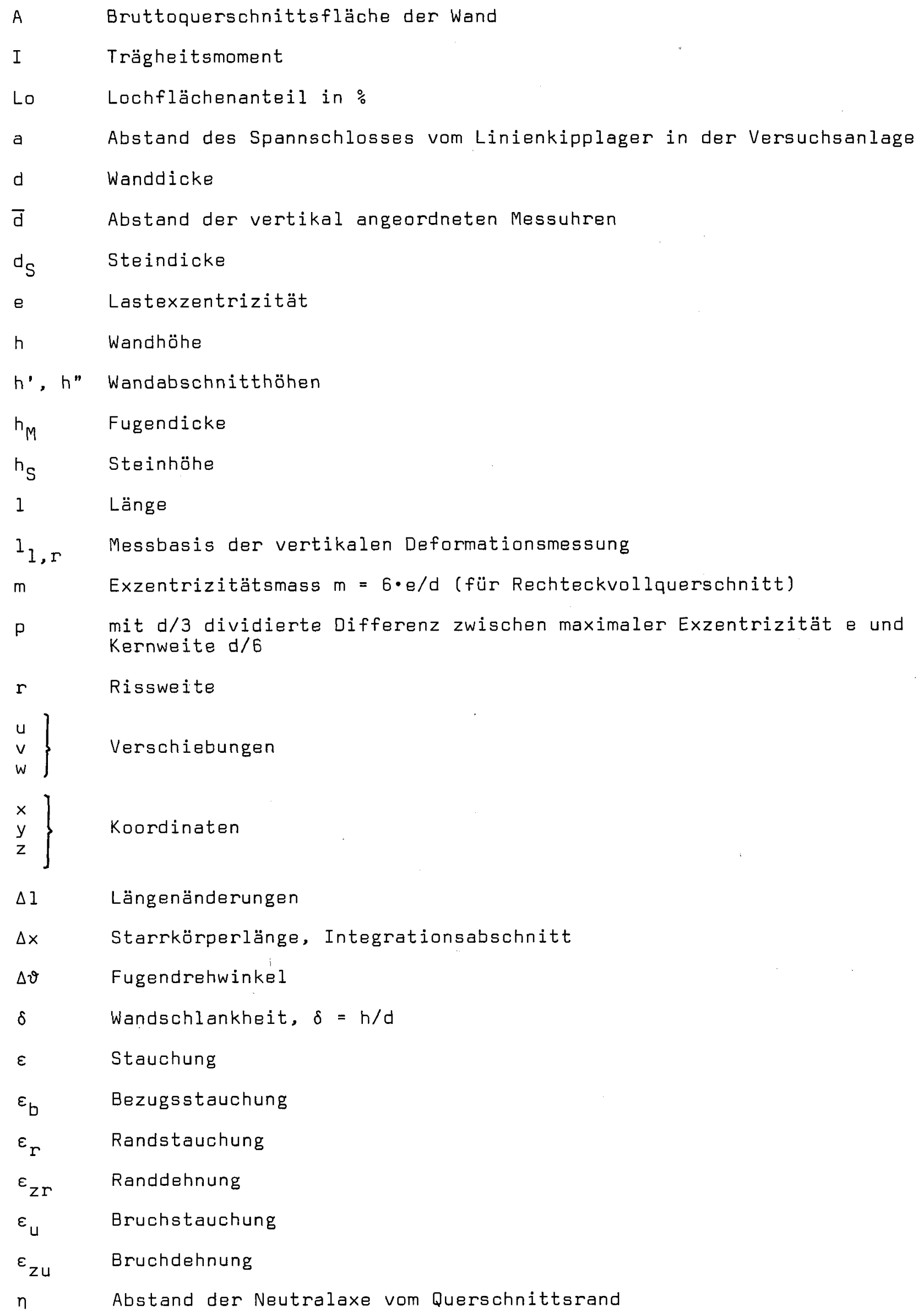




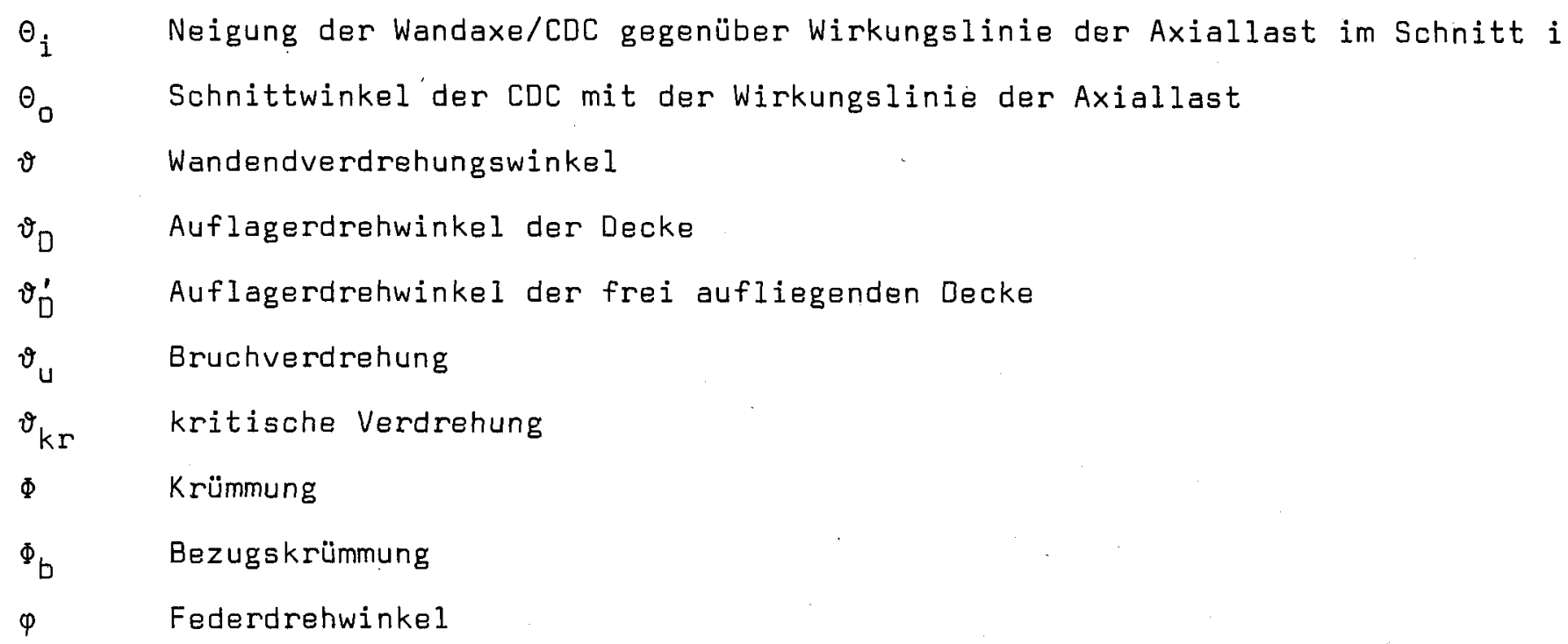

\section{Festigkeitswerte}

$\begin{array}{ll}\beta_{M} & \text { Mörteldruckfestigkeit } \\ \beta_{S} & \text { Steindruckfestigkeit } \\ \sigma_{U} & \text { Mauerwerksfestigkeit }\end{array}$

Spannungen und Kraftgrössen, Belastungen

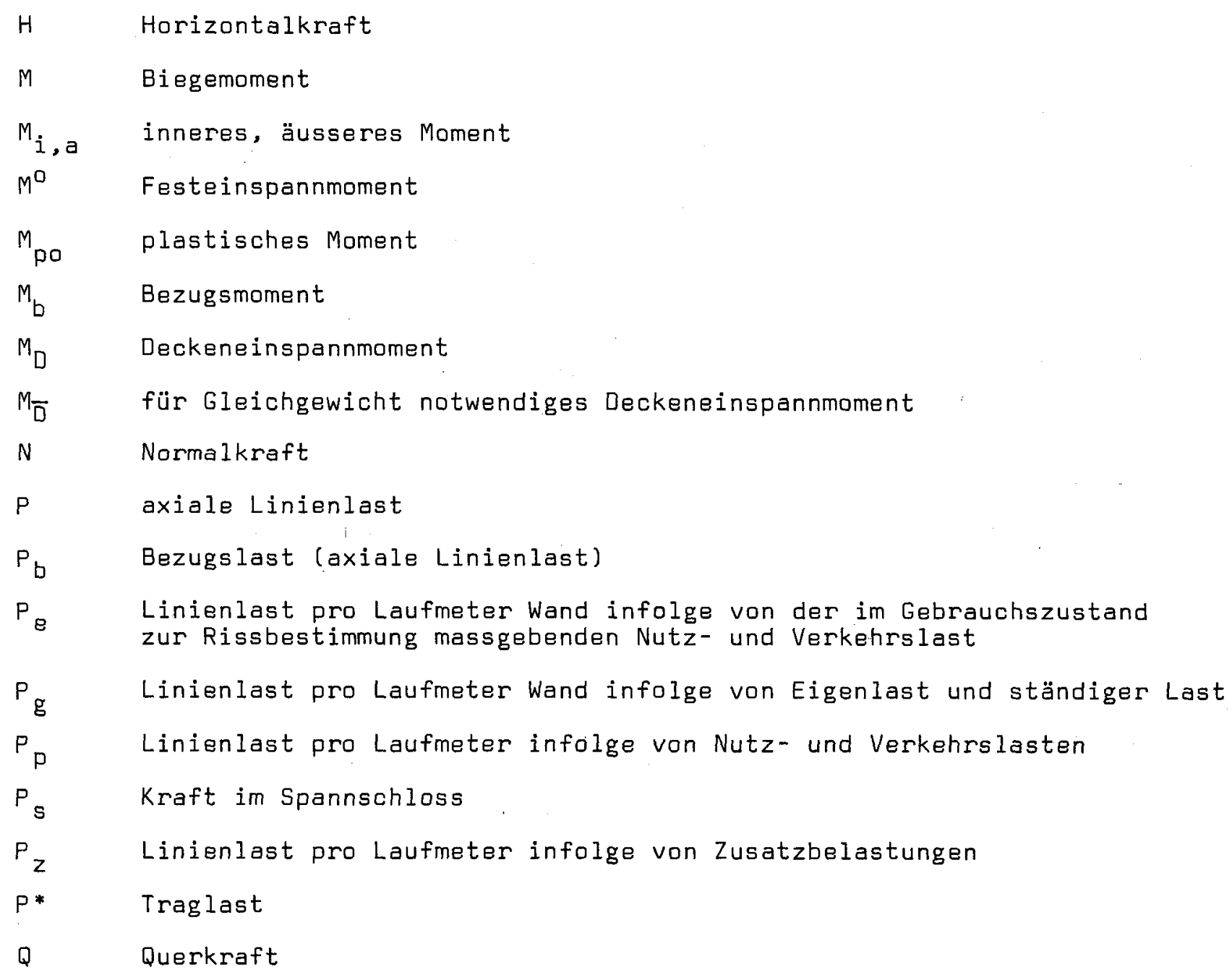


W Wandeinspannmoment

$\sigma_{B} \quad$ Bemessungsfestigkeit

$\sigma \quad$ Normalspannung

$\sigma_{b} \quad$ Bezugsspannung

$\sigma_{k r} \quad k r i t i s c h e$ Spannung

$\sigma_{m} \quad$ mittlere Spannung

$\sigma_{\mathrm{r}} \quad$ Randspanriung

$\sigma_{r b, r g}$ rechnerische Spannung

$\sigma^{*} \quad$ Traglastspannung

\section{Materialwerte}

E E-Modul von Mauerwerk

$E_{M} \quad E-M o d u l$ von Mörtel

$E_{S} \quad$ E-Modul des Mauersteins

s Saugfähigkeit des Mauersteins

\section{Verhältnisgrössen}

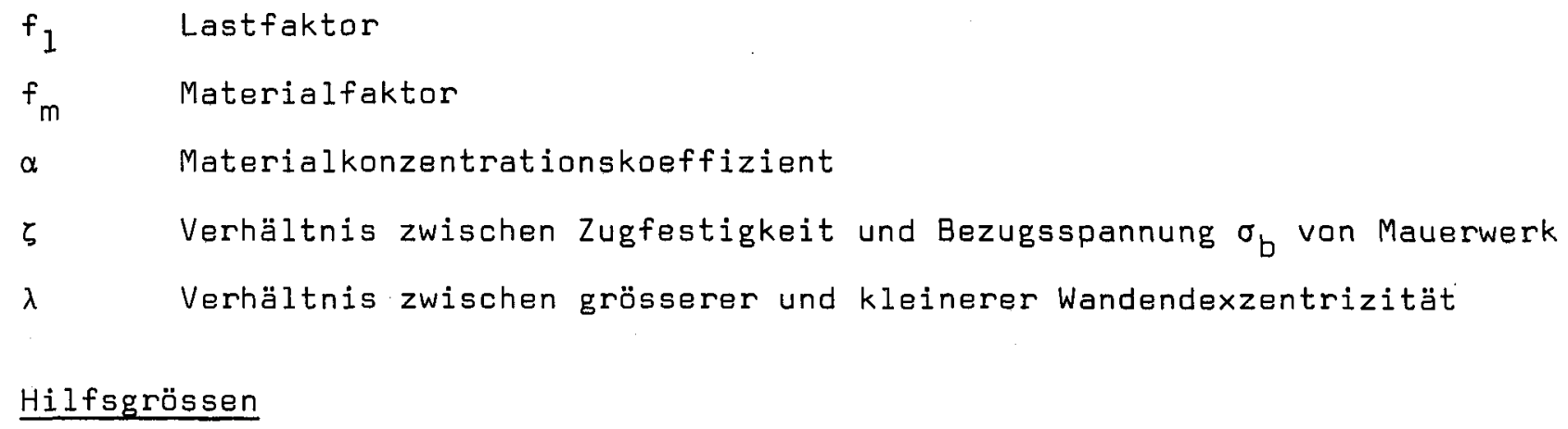

Hilfsgrössen

$C_{1}, \ldots 4$ Unbekannte

$\omega \quad$ Bezugswert $(\omega=\sqrt{\mathrm{P} / E T})$

\section{Fusszeiger}

A, B Punkte (z.B. $e_{A}$ : Exzentrizität im Punkt A) 


\section{Literaturverzeichnis}

[1] G.W. Anderson, R.J. Dayeh, J.W. Morgan: "The Interaction between Walls and Floor in a Structure of Loadbearing Brickwork", Dokumentation der 3. Internationalen Mauerwerkskonferenz, Essen, 1973.

[2] K. Angervo: "Ueber die Knickung und Tragfähigkeit eines exzentrisch gedrückten Pfeilers ohne Zugfestigkeit", Staatliche Technische Forschungsanstalt, Finnland, Publ. Nr. 26. Helsinki 1954.

[3] K. Angervo, A. Putkonen: "Erweiterung der. Theorie der Biegung eines Pfeilers ohne Zugfestigkeit", Staatliche Technische Forschungsanstalt, Finnland, Publ. Nr. 34, Helsinki 1961.

[4] E. Chwalla: "Theorie des aussermittig gedrückten Stabes aus Baustahl", Der Stahlbau, Oktober 1934.

[5] J. Colville, A.W. Hendry: "Tests of a Load Bearing Masonry Structure", 6th International Symposium on Load Bearing Brickwork, London 1977.

[6] A.J. Francis, C.B.Horman, L.E. Jerrems: "The Effect of the Joint Thickness and other Factors on the Compressive Strength of Brickwork", SIBMAC Proceedings, Stoke-on-Trent, England, 1970.

[7] R. Furler, B. Thürlimann: "Versuche über die Rotationsfähigkeit von Backsteinmauerwerk", Institut für Baustatik und Konstruktion, ETH Zürich, Bericht Nr. 7502-1, September 1977. Birkhäuser Verlag Basel und Stuttgart.

[8] R. Furler, B. Thürlimann: "Versuche über die Rotationsfähigkeit von Kalksandstein-Mauerwerk", Institut für Baustatik und Konstruktion, ETH Zürich, Bericht Nr. 7502-2, September 1980. Birkhäuser Verlag Basel und Stuttgart.

[9] Th.V. Galambos: "Structural Members and Frames", Prentice-Hall, Inc., Englewood Cliffs, N.J. 1968.

[10] G. Germanino, G. Macchi: "Experimental Research of a Frame-Idealisation for a Bearing-Wall Multi-story Structure", 6th International Symposium on Load Bearing Brickwork, London 1977.

[11] Glanville and Barnett: Mechanical Properties of Bricks and Brickwork Masonry", Department of Scientific and Industrial Research, Building Research. Special Report No. 22, London 1934.

[12] M.B. Grandet: "Mecanismes Physico-chimiques de l'Adherence entre la Terre Cuite et le Ciment", Dokumentation der 3. Internat. Mauerwerkskonfernz, Essen 1973.

[13] P. Haller: "Load Capacity of Brick Masonry". The Internat. Conference on Masonry Structural Systems, Austin. Texas, 1969.

[14] P. Haller: "Die Knickfestigkeit von Mauerwerk aus künstlichen Steinen", Sonderdruck aus Schweiz. Bauzeitung 1949, Hefte 38 und 39.

[15] P. Haller: "Die Porösität und die Kapillarität der Baustoffe aus gebranntem Ton und ihre Auswirkung auf die bautechnischen Eigenschaften", Extrait des Actes du $2 e$ Congrès Céramique International, Zürich 1950.

[16] H. Hilsdorf: "Untersuchungen über die Grundlagen der Mauerwerksfestigkeit", Materialprüfungsamt für das Bauwesen, TH München, Bericht Nr. $40,1965$.

[17] H. Hilsdorf: "Investigation into the Failure Mechanism of Brick Masonry Loaded in Axial Compression", The Internat. Conference on Masonry Structural Systems, Austin, Texas, 1969. 
[18] T. van Karman: "Untersuchungen über Knickfestigkeit", Mitteilungen und Forschungsarbeiten V.Q.I., 81, Berlin, 1910.

[19] C.0. Khoo, A.W. Hendry: "A Failure Criterion for Brickwork in Axial Compression", Dokumentation der 3. Internat. Mauerwerkskonferenz, Essen, 1973.

[20] K. Kirtschig: "Zur Tragfähigkeit von Mauerwerk bei mittiger Beanspruchung", Mitteilungen aus dem Institut für Baustoffkunde und Materialprüfwesen der TU Hannover, Heft 31, 1975.

[21] K. Kirtschig, P. Feldhaus, B. Gallenkemper, W. Schöner: "Aufbereitung eines Traglastverfahrens für den Mauerwerksbau". Mitteilungen aus dem Institut für Baustoffkunde und Materialprüfwesen der TU Hannover, Heft $30,1975$.

[22] H. Kreuger: "Brickwork Tests and Formulas for Calculation", The Clay Worker, London, July and August, 1917.

[23] M.G. Lay: "The Mechanics of Column Deflection Curves", Lehigh University, Fritz Engineering Laboratory, Report. No. 278.12, June 1964.

[24] H.P. Maurenbrecher, A.W. Hendry: "Aspects of the Strength and Fixity of the Joint between a Brick Wall and a Floor Slab", SIBMAC Proc., Stoke-on-Trent, England, 1970.

[25] L.E. Nevander: "Provningar av Tegelmurverk", Tegel No. 5, Stockholm, 1954 .

[26] B. Powell, H.R. Hodgkinson: "The Determination of Stress-Strain Relationship of Brickwork", Proceedings of the 4th Internat. Brick Masonry Conference, Brugge, 1976.

[27] R. Reinders: "Computerprogramm zur Optimierung der Wärmedämmung von Hochlochziegeln". Proceedings of the 4th Internat. Brick Masonry Conference, Brugge, 1976.

[28] F.E. Richart, A. Brandtzäg, R.L. Brown: "A Study of the Failure of Concrete under Combined Campressive Stresses", Bulletin 185, University of Illinois, Engineering. Experiment Station, 1928.

[29] S. Sahlin: "Interaction of Brick Masonry Walls and Concrete Slabs", The Internat. Conference on Masonry Structural Systems, Austin, Texas, 1969 .

[30] S. Sahlin: "Structural Masonry", Prentice-Hall, Inc., Englewood Cliffs, N.J., 1971.

[31] M.H. Satti, A.W. Hendry: "The Modulus of Rupture of Brickwork, Dokumentation der 3. Internat. Mauerwerkskonferenz, Essen, 1973.

[32] J. Schneider: "Merkmale des Sicherheitsproblems bei Bauwerken und Folgerungen", 9. Forschungskolloquium des Deutschen Aussschusses für Stahlbeton (DAfSt), ETH Zürich, 1978.

[33] Schweizerische Ziegelindustrie: "Backsteinmauerwerk", 1. Auflage, Zürich, 1977.

[34] Schweizerischer Ingenieur- und Architekten-Verein (SIA): "Norm SIA 113: Norm für die Berechnung und die Ausführung von Mauerwerk aus künstlichen und natürlichen Bausteinen". Ausgabe 1975, Zürich.

[35] Schweizerischer Ingenieur- und Architekten-Verein (SIA): "Vernehmlassungsentwurf der Norm SIA 177 (Norm für die Berechnung und die Ausführung von Mauerwerk aus künstlichen Bausteinen), Zürich, 1977.

[36] B.P. Sinha, A.W. Hendry: "An Investigation into the Behaviour of a Brick Cross-Wall Structure", 6th Internat. Symposium on Load Bearing Brickwork, London 1977. 


\section{Anhang Al}

Mauerwerksgattungen gemäss Norm SIA 177, Art. 443

\begin{tabular}{|c|c|c|c|}
\hline Bezeichnung & Mauerwerk aus & Qualität & Mörtel \\
\hline MBNV & Backstein & normal & verlängerter Mörtel \\
\hline MENC & Backstein & normal & Zementmörtel \\
\hline MBHC & Backstein & hochwertig & Zementmörtel \\
\hline MBSC & Backstein & Sonderqualität & Zementmörtel \\
\hline MKNV & Kalksandstein & normal & verlängerter Mörtel \\
\hline MKHV & Kalksandstein & hochwertig & verlängerter Mörtel \\
\hline MKHC & Kalksandstein & hochwertig & Zementmörtel \\
\hline MKSC & Kalksandstein & Sonderqualität & Zementmörtel \\
\hline MCLV & Zementstein & leicht & verlängerter Mörtel \\
\hline MCNC & Zementstein & normal & Zementmörtel \\
\hline MGLK & Gasbetonstein & leicht & Klebemörtel \\
\hline MGNK & Gasbetonstein & normal & Klebemörtel \\
\hline MGHK & Gasbetonstein & hochwertig & Klebemörtel \\
\hline
\end{tabular}

Die übliche Dosierung der Mörtelkomponenten in $\mathrm{kg} j \mathrm{je}^{3}$ fertigen Mörtels sind gemäss Norm SIA 177. Art. 4 514, wie folgt festgelegt:

- verlängerter Mörtel: $250 \mathrm{~kg} \mathrm{HK}+100 \mathrm{~kg} \mathrm{PC}$

- Zementmörtel : $\quad 300 \div 450 \mathrm{~kg} \mathrm{PC}$

- Klebemörtel : nach den Vorschriften der Hersteller. 
Anhang A2: Linear elastisches Material mit Zugfestigkeit

Unter der Voraussetzung des linear elastischen Materials mit Zugfestigkeit (Bild 4.9) ergeben sich für den ungerissenen Querschnitt die gleichen analytischen Beziehungen wie für das linear elastische Material ohne Zugfestigkeit (Bild 4.1): Die Bezugsgrössen $P_{b}, M_{b}$ und $\Phi_{b}$ sind durch die Gl. (4.1) gegeben.

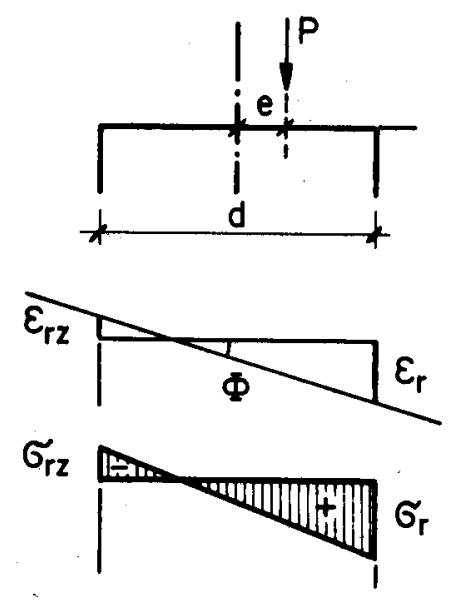

$$
\begin{aligned}
& \frac{M}{M_{b}}=\frac{\Phi}{\Phi_{b}} \\
& \frac{e}{d}=\frac{1}{6} \cdot \frac{\Phi}{\Phi_{b}} \cdot \frac{P_{b}}{P} \\
& \frac{P}{P_{b}}=\frac{1}{2} \cdot\left(\frac{\sigma_{r}}{\sigma_{b}}+\frac{\sigma_{r z}}{\sigma_{b}}\right)
\end{aligned}
$$

mit den Gültigkeitsbereichen:

$$
\begin{aligned}
& \frac{M}{M_{b}} \leq \frac{P}{P_{b}}+\zeta \\
& \frac{e}{d} \leq \frac{1}{6} \cdot\left(1+\frac{\zeta}{P / P_{b}}\right)
\end{aligned}
$$

Für den gerissenen Querschnitt lauten die Beziehungen wie folgt:

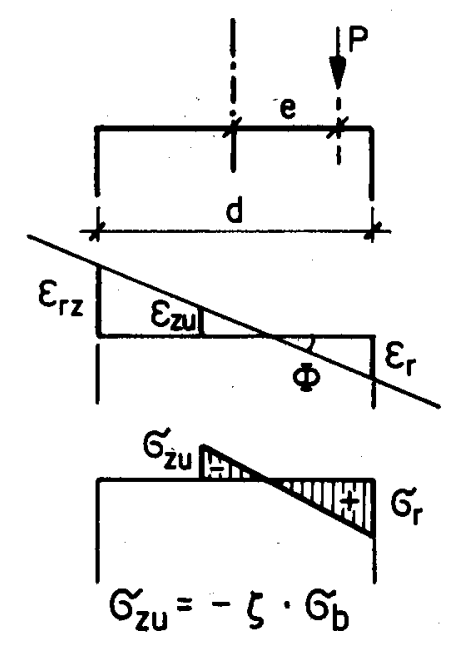

$$
\begin{aligned}
& \frac{P}{P_{b}}=\frac{\left(\frac{\sigma_{F}}{\sigma_{b}}\right)^{2}-\zeta^{2}}{4 \cdot \frac{\Phi}{\Phi_{b}}} \\
& \frac{e}{d}=\frac{1}{2}-\frac{1}{6} \cdot \frac{\sigma_{r}}{\sigma_{b}} \cdot \frac{\Phi_{b}}{\Phi}+\frac{\zeta^{2} \cdot\left\{\frac{\sigma_{r}}{\sigma_{b}}+\zeta\right\}}{12 \cdot \frac{P}{P_{b}} \cdot\left(\frac{\Phi}{\Phi_{b}}\right)^{2}} \\
& \frac{\Phi}{\Phi_{b}}=\frac{1}{2} \cdot\left\{\frac{\varepsilon_{r}}{\varepsilon_{b}}-\frac{\varepsilon_{r z}}{\varepsilon_{b}}\right\} \\
& \frac{M_{b}}{M_{b}}=6 \cdot \frac{e}{d} \cdot \frac{P}{P_{b}}
\end{aligned}
$$




\section{Anhang A3: Linear elastisch-ideal plastisches Material ohne Zugfestigkeit}

Bei der Angabe der analytischen Beziehungen der e- $\Phi-K u r v e n$ und der Interaktion $M-P$ sind die nachfolgenden vier Fälle zu unterscheiden:

Die Bezugsgrössen $P_{b}, M_{b}$ und $\Phi_{b}$ sind durch die Gl. (4.1) gegeben.

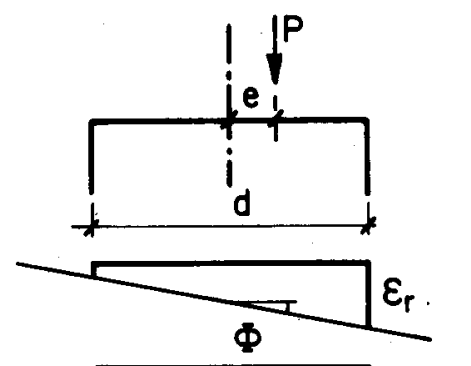

$$
\begin{aligned}
& \frac{M}{M_{b}}=\frac{\Phi}{\Phi_{b}} \\
& \frac{e}{d}=\frac{1}{6} \cdot \frac{\Phi}{\Phi_{b}} \cdot \frac{P}{P_{b}}
\end{aligned}
$$

wobei:

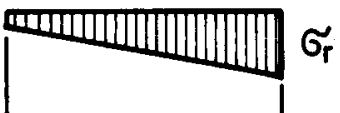

$2 \cdot \frac{\Phi}{\Phi} \leq \frac{\varepsilon_{r}}{\varepsilon_{b}} \leq 1$

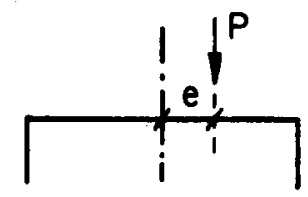

$\frac{P}{P_{b}}=\frac{1}{2} \cdot \frac{\Phi}{\Phi} \cdot\left\{\frac{\varepsilon_{r}}{\varepsilon_{b}}-\frac{1}{2}-\frac{1}{2} \cdot\left(\frac{\varepsilon_{r}}{\varepsilon_{b}}\right)^{2}\right\}-\frac{\Phi}{\Phi_{b}}+\frac{\varepsilon_{r}}{\varepsilon_{b}}$

$\frac{M}{M_{b}}=-\frac{3}{4} \cdot \frac{\Phi}{\Phi} \cdot\left(1-\frac{\varepsilon_{r}}{\varepsilon_{b}}\right)^{2}+\frac{1}{4} \cdot\left(\frac{\Phi_{b}}{\Phi}\right)^{2} \cdot\left(\frac{\varepsilon_{r}}{\varepsilon_{b}}-1\right)+\frac{\Phi}{\Phi_{b}}$

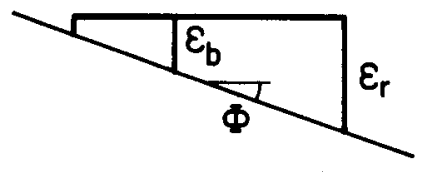

wobei :

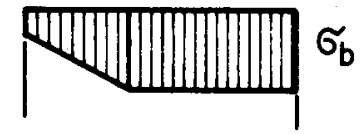

$\frac{\varepsilon_{r}}{\varepsilon_{b}} \geq$

$2 \cdot \frac{\Phi}{\Phi_{b}} \leq \frac{\varepsilon_{r}}{\varepsilon_{b}}$

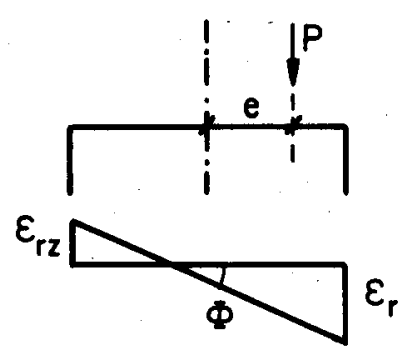

$\frac{P}{P_{b}}=\frac{\left(\sigma_{r} / \sigma_{b}\right)^{2}}{4 \cdot \frac{\Phi}{\Phi_{b}}}$

$\frac{M}{M_{b}}=3 \cdot \frac{P}{P_{b}} \cdot\left(1-\sqrt{\frac{4}{9} \cdot \frac{P}{P_{b}} \cdot \frac{\Phi_{b}}{\Phi}}\right)$

wobei:

$\frac{\varepsilon_{r}}{\varepsilon_{b}} \leq 1$

$2 \cdot \frac{\Phi}{\Phi_{b}} \geq \frac{\varepsilon_{r}}{\varepsilon_{b}}$ 


$$
\begin{aligned}
\frac{P}{P_{b}} & =\frac{1}{2} \cdot \frac{\Phi_{b}}{\Phi} \cdot\left\{\frac{\varepsilon r}{\varepsilon_{b}}-\frac{1}{2}\right\} \\
\frac{M}{M_{b}} & =\frac{3}{2} \cdot\left(\frac{\Phi_{b}}{\Phi}\right)^{2} \cdot\left\{\frac{1}{2} \cdot \frac{\varepsilon_{r}}{\varepsilon_{b}}-\frac{1}{2} \cdot\left(\frac{\varepsilon_{r}}{\varepsilon_{b}}\right)^{2}-\frac{1}{6}\right\} \\
& +3 \cdot \frac{\Phi_{b}}{\Phi} \cdot\left\{\frac{1}{2} \cdot \frac{\varepsilon_{r}}{\varepsilon_{b}}-\frac{1}{4}\right\} \\
2 \cdot \frac{\Phi}{\Phi_{b}} & \geq \frac{\varepsilon_{r}}{\varepsilon_{b}} \geq 1
\end{aligned}
$$


Anhang A4: Krümmungs- und Interaktions-Beziehungen bel zwel extremen Lochungen $A$ und $B$ (vgl. Bild 4.16)

Für die Bestimmung der nachfolgend aufgeführten analytischen Beziehungen wurde ein linear elastisches Material ohne Zugfestigkeit vorausgesetzt. Je nach Lochung (Bild 4.16) sind vier (bei Lochung A) bzw. zwei Fälle (bei Lochung B) zu unterscheiden. Die Bezugsgrössen $P_{b}$, $M_{b}$ und $\Phi_{b}$ sind durch die Gl. (4.1) gegeben.

\section{Lochung $A$}
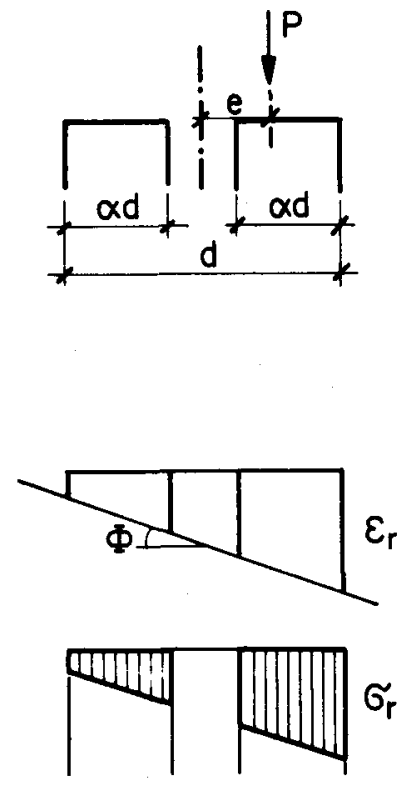

$$
\begin{aligned}
& \frac{P}{P_{b}}=\left(\frac{\sigma_{r}}{\sigma_{b}}-\frac{\Phi}{\Phi_{b}}\right) \cdot 2 \alpha \\
& \frac{M}{M_{b}}=\frac{\Phi}{\Phi_{b}} \cdot\left(3+4 \alpha^{2}-6 \alpha\right) \cdot 2 \alpha
\end{aligned}
$$

wobei: $2 \cdot \frac{\Phi}{\Phi_{b}} \leq \frac{\varepsilon_{r}}{\varepsilon_{b}}$

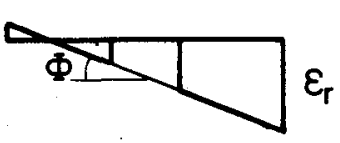

$$
\frac{P}{P_{b}}=\frac{1}{4} \cdot\left(\frac{\sigma_{r}}{\sigma_{b}}\right)^{2} \cdot \frac{\Phi_{b}}{\Phi}-(1-2 \alpha) \cdot\left(\frac{\sigma_{r}}{\sigma_{b}}-\frac{\Phi}{\Phi_{b}}\right)
$$

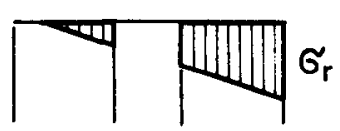

$$
\begin{aligned}
& \frac{M}{M_{b}}=\frac{1}{4} \cdot\left(\frac{\sigma_{r}}{\sigma_{b}}\right)^{2} \cdot\left(\frac{\Phi b}{\Phi}\right)^{2} \cdot\left(3 \cdot \frac{\Phi}{\Phi_{b}}-\frac{\sigma_{F}}{\sigma_{b}}\right)-(1-2 \alpha)^{3} \cdot \frac{\Phi}{\Phi_{b}} \\
& \text { mit: } \frac{\varepsilon_{r}}{b} \leq 2 \cdot \frac{\Phi}{\Phi_{b}} \leq \frac{1}{1-\alpha} \cdot \frac{\varepsilon_{r}}{\varepsilon_{b}}
\end{aligned}
$$

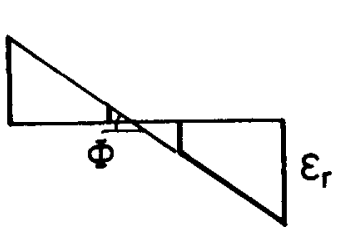

$$
\frac{P}{P_{b}}=\alpha \cdot \frac{\sigma_{r}}{\sigma_{b}}-\alpha^{2} \cdot \frac{\Phi}{\Phi_{b}}
$$

$$
\frac{M}{M_{b}}=3 \alpha \cdot(1-\alpha) \cdot \frac{\sigma_{r}}{\sigma_{b}}-\alpha^{2} \cdot(3-4 \alpha) \cdot \frac{\Phi}{\Phi_{b}}
$$

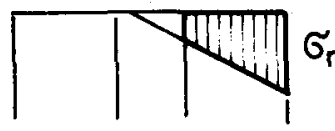

mit :

$$
\frac{1}{1-\alpha} \cdot \frac{\varepsilon_{r}}{\varepsilon_{b}} \leq 2 \cdot \frac{\Phi}{\Phi_{b}} \leq \frac{1}{\alpha} \cdot \frac{\varepsilon_{r}}{\varepsilon_{b}}
$$




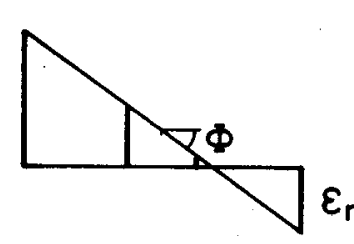

$\frac{P}{P_{b}}=\frac{\left(\sigma_{r} / \sigma_{b}\right)^{2}}{4 \cdot \frac{\Phi}{\Phi_{b}}}$

$\frac{M}{M_{b}}=\left(3-\frac{\sigma_{r}}{\sigma_{b}} \cdot \frac{\Phi}{\Phi}\right) \cdot \frac{P}{P_{b}}$

1

mit :

$\frac{1}{\alpha} \cdot \frac{\varepsilon_{F}}{\varepsilon_{b}} \leq 2 \cdot \frac{\Phi}{\Phi_{b}}$

Lochung $B$

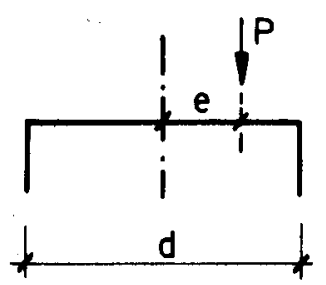

$\frac{P}{P_{b}}=2 \alpha \cdot\left(\frac{\sigma_{r}}{\sigma_{b}}-\frac{\Phi}{\Phi_{b}}\right)$

$\frac{M}{M_{b}}=2 \alpha \cdot \frac{\Phi}{\Phi_{b}}$

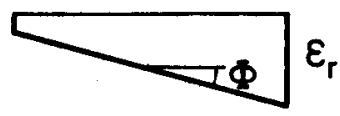

mit :

$2 \cdot \frac{\Phi}{\Phi_{b}} \leq \frac{\varepsilon_{F}}{\varepsilon_{b}}$

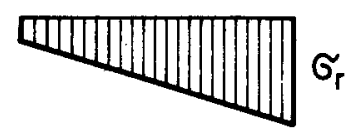

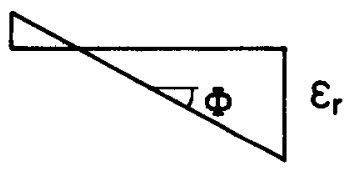

$\frac{P}{P_{b}}=2 \alpha \cdot \frac{\left(\sigma_{r} / \sigma_{b}\right)^{2}}{4 \cdot\left(\frac{\Phi}{\Phi_{b}}\right)}$

$\frac{M}{M_{b}}=\left(3-\frac{\sigma_{r}}{\sigma_{b}} \cdot \frac{\Phi}{\Phi}\right) \cdot \frac{P}{P_{b}}$

WU. $\sigma_{r}$

mit:

$2 \cdot \frac{\Phi}{\Phi_{b}} \geq \frac{\varepsilon_{F}}{\varepsilon_{b}}$ 
Anhang A5: Bemessungsverfahren für Backstein- und Kalksandstein-Mauerwerk unter Axiallast und Biegung

Inhaltsverzeichnis

1. Gültigkeit

1. 1 Mauerwerksqualität

1.2 Steinlochung

2. Grundsätze für die Bemessung

2.1 Bemessung auf Grenzzustände

2.2 Bemessungsverfahren

2.3 Bemessungslasten

2.4 Bemessungsfälle

3. Nachweis der Bruchsicherheit

3.1 Grundsatz

3.2 Bestimmung der Festigkeit $\sigma_{B}$

3.3 Bestimmung der rechnerischen Spannung $\sigma_{r b}$

3.4 Auflagerverdrehung der Decken

4. Nachweis der Gebrauchsfähigkeit

4.1 Grundsatz

4.2 Kriterien der Gebrauchsfähigkeit

4.3 Ermittlung der rechnerischen Spannung $\sigma_{r g}$

4.4 Ermittlung der theoretischen Rissweiten

4.5 Auflagerverdrehung der Decken infolge von Schwind- und Temperaturverformungen 
1. Gültigkait

Die Richtlinie gilt für die Bemessung von tragenden Mauerwerkswänden (Backsteine oder Kalksandsteine) unter Axiallast und Biegung.

\subsection{Mauerwerksarten}

Die nachfolgenden Mauerwerksbezeichnungen entsprechen der Norm SIA 177, Art. 4 43.

\section{$1=1$ 1 1 B Backs}

Einstein- und Verband-Mauerwerk:

- MBNC, MBNV

- $M B H C$

- MBSC

\subsubsection{Kalksandsteinmauerwerk}

Einstein- und Verband-Mauerwerk

- MKNV

- MKHC, MKHV

- MKSC

\subsection{Steinlochung}

Die nachfolgenden Bemessungsdiagramme gelten für Steine mit folgendem Lochflächenanteil Lo:

- Backsteinmauerwerk

- Kalksandsteinmauerwerk

$$
\begin{aligned}
& L_{0}^{50}=50 \% \\
& L_{0}^{20}=20 \%
\end{aligned}
$$

Damit bei. der Bemessung diese Diagramme auch für Steine mit abweichendem Lochflächenanteil Lo* verwendet werden können, sind die rechnerischen Spannungen $\sigma_{\mathrm{rb}}$ und $\sigma_{\mathrm{rg}}$ gemäss 3.3.3 umzurechnen. Diese Umrechnung kann für die folgenden Lochflächenanteile verwendet werden:

- Backsteinmauerwerk

$30 \% \leq L^{*} \leq 60 \%$

- Kalksandsteinmaverwerk

$0 \% \leq L^{*} \leq 30 \%$

2. Grundsätze für die Bemessung

\subsection{Bemessung auf Grenzzustände}

Grundsätzlich sind die Mauerwerkswände so zu bemessen und durchzubilden, dass sie

- eine ausreichende Bruchsicherheit aufweisen (Tragfähigkeit)

- unter Gebrauchslasten ein normales Verhalten zeigen (Gebrauchsfähigkeit) und auftretende Verformungen mit Einschluss der Kriech-, Schwind- und Temperaturverformungen keinen nachteiligen Einfluss auf die Nutzung haben. 


\subsection{Bemessungsverfahren}

Alle angegebenen Spannungen $\left(\sigma_{B}, \sigma_{r b}, \sigma_{r g}\right)$ sind auf die Bruttoquerschnittsfläche $A$ der Wand bezogen. Es handelt sich demnach um Bruttospannungen. Unterschiedliche Lochflächen kännen gemäss 1.2 bzw. 3.3 .3 behandelt werden.

\subsection{Bemessungslasten}

Die Mauerwerkswand ist sowohl für Hauptlasten als auch für Haupt- und Zusatzlasten zu bemessen (SIA Norm 177, Art. 3112 und Art 3 113). Liegen besondere Verhältnisse vor, so ist eine sinngemäss abgeänderte Aufteilung in Haupt -und Zusatzlasten vorzunehmen.

\subsection{Bemessungsfälle}

Folgende Bemessungsfälle werden unterschieden:

- Fälle E:

Axiale Linienlast mit konstanten, vorgegebenen Exzentrizitäten am oberen und unteren Wandende.

E1

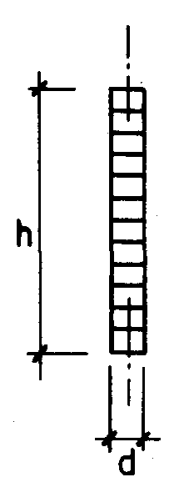

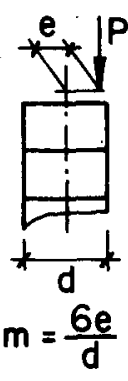

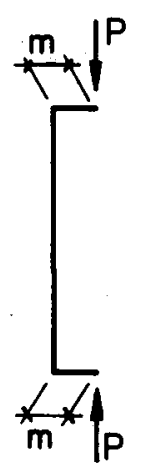

E2

E3
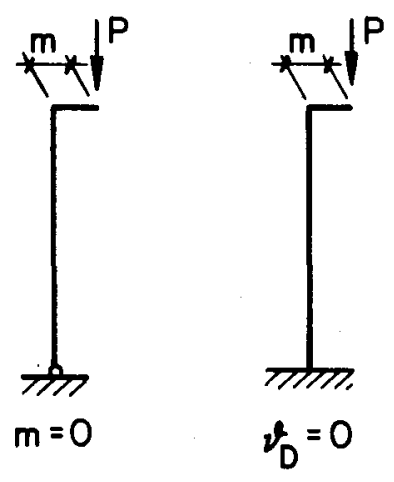

- Fälle V: Axiale Linienlast und Verdrehungen der Deckenauflager, die sich aus dem Zusammenwirken von Decke und Wänden ergeben.

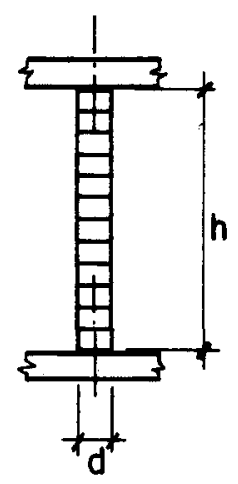

$$
\text { V1 V2 V3 }
$$

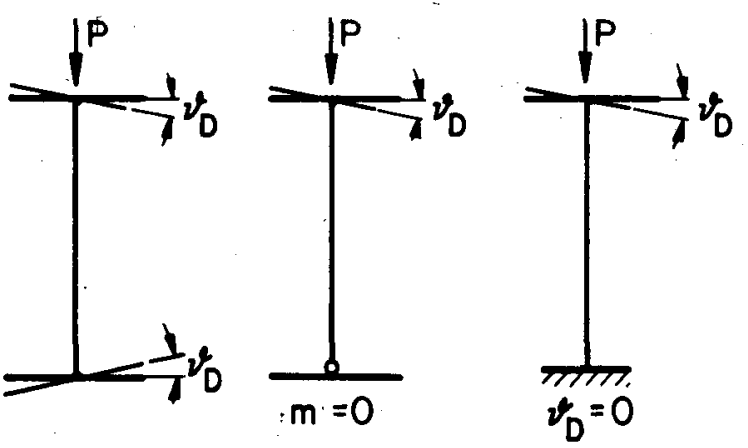


Für die Bemessung ist von den angegebenen Fällen derjenige zu wählen, welcher dem zu untersuchenden Fall am nächsten liegt. Wenn nötig, darf linear interpoliert werden.

\section{Nachweis der Bruchsicherheit}

\subsection{Grundsatz}

Eine ausreichende Bruchsicherheit ist vorhanden, wenn die nach 3.2 ermittelte Bemessungsfestigkeit $\sigma_{B}$ die auf den Bruttoquerschnitt bezogene rechnerische Spannung $\sigma_{r b}$ infolge der um einen Lastfaktor vergrösserten Lasten übersteigt:

$$
\sigma_{B} \geq \sigma_{r b}
$$

\subsection{Bestimmung der Festigkeit $\sigma_{B}$}

\subsubsection{Vorgehen}

Bemessungsfall E:

- Schlankheit $\delta$

- Exzentrizitätsmass m

- Bestimmung des Bemessungsfalles E (nach 2.4)

- Bestimmung von $\sigma_{B}$ aus den Bemessungs-Diagrammen $\mathrm{BE}$
Bemessungsfall V:

- Schlankheit $\delta$

- Auflagerwinkel $\vartheta_{D}$ der Decken (nach 3.4)

- Bestimmung des Bemessungsfalles $V$ (nach 2.4)

- Bestimmung von $\sigma_{B}$ aus den Bemessungs-Diagrammen BV

Die Schlankheit $\delta$ wird gemäss untenstehender Darstellung ermittelt:

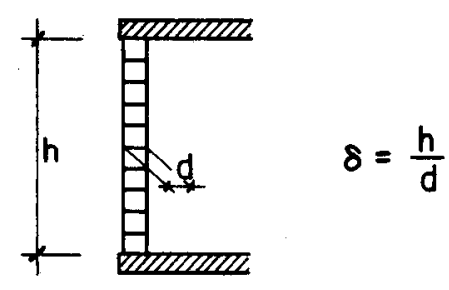

Das Exzentrizitätsmass m stellt die auf die Kernweite des Rechteckvollquerschnittes ( $k=d / 6)$ bezogene Exzentrizität dar.

\subsubsection{Berücksichtigung der Mauerwerksqualität}

Die Werte der Bemessungs-Diagramme BE und BV sind für MBHC bzw. MKHC gültig. Für die andern Mauerwerksqualitäten (siehe 1.1) sind die Werte der BemessungsDiagramme mit den angegebenen $\gamma$-Werten je nach Bemessungsfall wie folgt umzurechnen: 
Bemessungsfall E

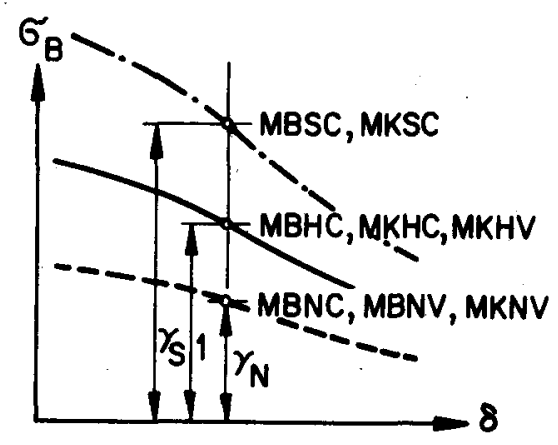

- Backsteinmauerwerk

- Kalksandsteinmauerwerk

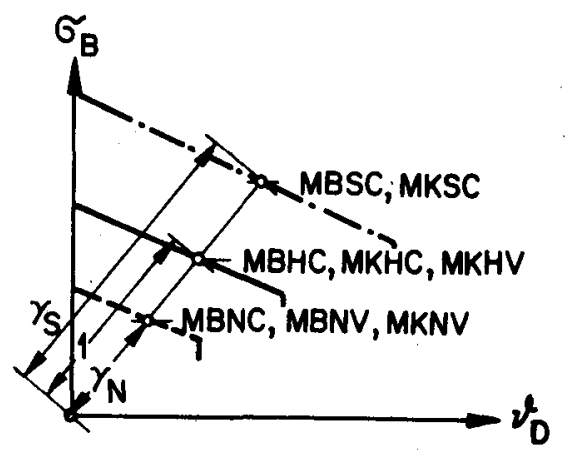

\subsection{Bestimmung der rechnerischen Spannung $\sigma$ rb}

3.3.1 für Hauptlasten

$$
\sigma_{r b}=\frac{1.4 \cdot\left(P_{g}+P_{p}\right)}{A}
$$

bzw. bei günstiger (entlastender) Wirkung von $P_{g}$ :

$$
\sigma_{r b}=\frac{0.8 P_{g}+1.4 P_{P}}{A}
$$

3.3.2 für Haupt- und Zusatzlasten

$$
\sigma_{r b}=\frac{1.2 \cdot\left(P_{g}+P_{p}+P_{z}\right)}{A}
$$

bzw. bei günstiger (entlastender) Wirkung von $\mathrm{P}_{\mathrm{g}}$ :

$$
\sigma_{r b}=\frac{0.8 \cdot P_{g}+1.2 \cdot\left(P_{P}+P_{z}\right)}{A}
$$

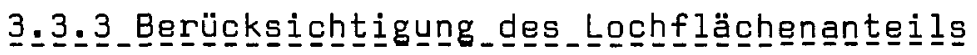

Werden für die zu bemessende Mauerwerkswand Steine mit einem von 50\% (Backsteine) bzw. $20 \%$ (Kalksandsteine) abweichenden Lochflächenanteil Lo* verwendet, so ist die rechnerische spannung $\sigma_{\mathrm{rb}}$ wie folgt umzurechnen und mit der Bemessungsfestigkeit $\sigma_{B}$ (nach 3.2.1 und 3.2.2) zu vergleichen:

- Backsteinmauerwerk

$$
\sigma_{r b}^{*}=\sigma_{r b} \cdot \frac{1-L_{0} 50}{1-L_{0}^{*}}
$$

- Kalksandsteinmauerwerk

$$
\sigma_{\Gamma b}^{*}=\sigma_{r b} \cdot \frac{1-L o^{20}}{1-L \sigma^{*}}
$$


$\sigma_{\text {rb }}:$ rechnerische Spannung nach 3.3

$\sigma_{\mathrm{rb}}^{*} \quad$ : umgerechnete rechnerische Spannung

Lo $^{50}$ : Lochflächenanteil 50\%

Lo 20 : Lochflächenanteil $20 \%$

Lo* : abweichender Lochflächenanteil in
Backsteinmauerwerk
$30 \% \leq L^{*} \leq 60 \%$
Kalksandsteinmauerwerk
$0 \% \leq L^{*} \leq 30 \%$

\subsection{Auflagerverdrehung der Decken}

Die in den Backsteinwänden eingebundenen Decken erfahren durch die Wände eine Einspannung. Der Winkel der Deckenauflagerverdrehung $\vartheta_{D}$ stellt sich so ein, dass der Gesamteinspannwiderstand $W$ der Backsteinwand gleich dem Einspannmoment $M_{D}$ der Decke ist:

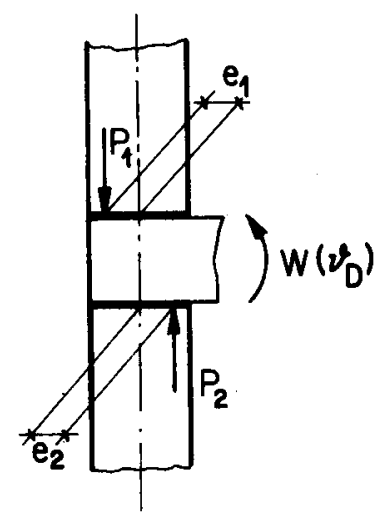

$$
\begin{array}{ll}
\text { Wand: } & W=W\left(v_{D}\right)=P_{1} \cdot\left(e_{1}\left(v_{D}\right)+e_{2}\left(v_{D}\right)\right) \\
\text { Decke: } & M_{D}=M_{D}\left(v_{D}\right)
\end{array}
$$

Gleichgewicht: $W\left(v_{D}\right)=M_{D}\left(v_{D}\right)$

Die Kurven $\varepsilon_{1}\left(\vartheta_{D}\right)$ und $e_{2}\left(\vartheta_{D}\right)$ können purktweise für m-Werte von $0.5,1.0,1.5$ und 2.0 aufgetragen werden: Bei gegebener Last, Schlankheit $\delta$ und Bemessungsfall V kann die zu einem m- bzw. e-Wert gehörende Verdrehung des Deckenauflagers aus dem Diagramm DE bestimmt werden. $\sigma_{\text {rb }}$ ist gemäss $3.3 \mathrm{zu}$ bestimmen. Für $\mathrm{m}$ ist höchstens der Wert 2.0 einzusetzen. Durch Addition der beiden Kurven $e_{1}\left(\vartheta_{D}\right)$ und $e_{2}\left(\vartheta_{D}\right)$ und Multiplikation mit der Linienlast in der oberen Wand erhält man $W\left(\vartheta_{D}\right)$.

Das Einspannmoment $M_{D}\left(\vartheta_{D}\right)$ kann ebenfalls in Abhängigkeit von $\vartheta_{D}$ bestimmt werden. Kriechen kann berücksichtigt werden. Im Schnittpunkt von $W\left(\vartheta_{D}\right)$ und $M_{D}\left(\vartheta_{D}\right)$ ist die Gleichgewichtsbedingung $W\left(\vartheta_{D}\right)=M_{D}\left(\vartheta_{D}\right)$ erfüllt; damit ist der Auflagerdrehwinkel bestimmt.

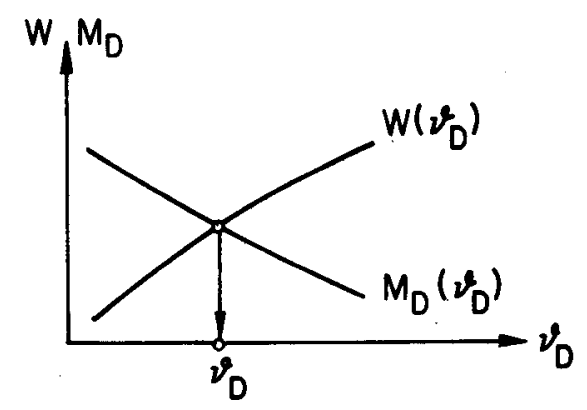




\subsection{Grundsatz}

Die Gebrauchsfähigkeit ist gewährleistet, wenn unter den massgebenden Lasten, Verdrehungen der Deckenauflager sowie Zwängungen infolge von Kriech-, Schwindund Temperaturverformungen der Decken keine Risse auftreten bzw. nur solche, die keine Folgeschäden nach sich ziehen und das Aussehen der Wand nicht beeinträchtigen. Es handelt sich dabei um horizontale Risse in den Lagerfugen sowie zwischen Wand und Decke.

Rissbildungen in Backsteinwänden können nicht nur durch Zwängungen und Verdrehungen der Deckenauflager entstehen. Beim Konstruieren und Bemessen von Backsteinbauten sind die übrigen Konstruktionsregeln zur Vermeidung von Rissen selbstverständlich zu beachten.

\subsection{Kriterien der Gebrauchsfähigkeit}

Im Gebrauchszustand sollen in der Backsteinwand keine bzw. keine unzulässigen Risse auftreten. Als unzulässig werden Risse bezeichnet, welche Folgeschäden nach sich ziehen und/oder das Aussehen der Wand beeinträchtigen. Die Möglichkeit des Auftretens von Folgeschäden ist unter Berücksichtigung der betreffenden Wandkonstruktion und der Lage in bezug auf die möglichen Witterungseinflüsse zu beurteilen.

In der Regel lassen sich die obigen Forderungen erfüllen, wenn die folgenden theoretischen Rissweiten (Richtwerte) nicht überschritten werden:

\begin{tabular}{|c|c|c|c|c|}
\hline Lage bezügl. Witterungseinfluss & & $\begin{array}{l}\text { Verputztes Mau } \\
\text { abgesackt oder } \\
\text { dünner Putz bzw. } \\
\text { feine Oberfläche }\end{array}$ & $\begin{array}{l}\text { uerwerk } \\
\text { dicker Putz } \\
\text { bzw. grobe } \\
\text { Oberfläche }\end{array}$ & $\begin{array}{l}\text { Unverputztes Mauerwerk, } \\
\text { Sichtmauerwerk }\end{array}$ \\
\hline $\begin{array}{l}\text { Vor Witterungseinflüssen voll- } \\
\text { kommen geschützt }\end{array}$ & $\begin{array}{l}\text { A } \\
\text { B } \\
\end{array}$ & $\begin{array}{l}0.10 \mathrm{~mm} \\
0.15 \mathrm{~mm} \\
\end{array}$ & $\begin{array}{l}0.15 \mathrm{~mm} \\
0.20 \mathrm{~mm} \\
\end{array}$ & $0.15 \mathrm{~mm}$ \\
\hline $\begin{array}{l}\text { Direkter Schlagregen durch aus- } \\
\text { ladendes Vordach weitgehend } \\
\text { abgehalten }\end{array}$ & $\begin{array}{l}\text { A } \\
\text { B }\end{array}$ & $\begin{array}{l}0.05 \mathrm{~mm} \\
0.10 \mathrm{~mm} \\
\end{array}$ & $\begin{array}{l}0.10 \mathrm{~mm} \\
0.15 \mathrm{~mm} \\
\end{array}$ & $0.10 \mathrm{~mm}$ \\
\hline $\begin{array}{l}\text { Den Witterungseinflüssen } \\
\text { ungeschützt ausgesetzt }\end{array}$ & $\begin{array}{l}\text { A } \\
\text { B } \\
\end{array}$ & $\begin{array}{l}0.00 \mathrm{~mm} \\
0.05 \mathrm{~mm} \\
\end{array}$ & $\begin{array}{l}0.05 \mathrm{~mm} \\
0.10 \mathrm{~mm} \\
\end{array}$ & $0.05 \mathrm{~mm}$ \\
\hline $\begin{array}{l}\text { Extreme Lage bezüglich } \\
\text { Witterung }\end{array}$ & $\begin{array}{l}\text { A } \\
\text { B }\end{array}$ & $\begin{array}{l}0.00 \mathrm{~mm} \\
0.00 \mathrm{~mm}\end{array}$ & $\begin{array}{l}0.00 \mathrm{~mm} \\
0.05 \mathrm{~mm}\end{array}$ & $0.00 \mathrm{~mm}$ \\
\hline
\end{tabular}

A: Massgebliche Schwind- und Kriechverformungen der Decken nach Auftragen des Verputzes

B: Schwinden und Kriechen der Decken im wesentlichen abgeklungen beim Auftragen des Verputzes 


\subsection{Ermittlung der rechnerischen Spannung $\sigma_{r g}$}

$$
\sigma_{r g}=\frac{P_{g}+P_{e}}{A}
$$

Sofern Steine verwendet werden, die einen von $50 \%$ (Backsteine) bzw. von $20 \%$ (Kalksandsteine) abweichenden Lochflächenanteil aufweisen, ist $\sigma_{r g}$ entsprechend 3.3.3 umzurechnen.

\subsection{Ermittlung der theoretischen Rissweiten}

4.4.1 Einfluss der Mauerwerksqualität

Die Mauerwerksqualität ist für die Rissweiten ohne Bedeutung. Die Begrenzung der Diagramme GV ist jedoch entsprechend 3.2.2 abzuändern.

$\underline{4}: \underline{4}: \underline{2}-\underline{F} \underline{a} \underline{1} \underline{\underline{E}}-\underline{E}$

- Bestimmung von $m$ und $\delta$.

- Ermittlung der Wandverdrehung infolge der massgebenden Lasten aus den Diagrammen DE 1,2 und 3 sowie der Auflagerverdrehung infolge von Schwindund Temperaturverformungen nach 4.5 .

- Wahl des Bemessungsfalles $V$ aufgrund der totalen Auflagerverdrehung und Bestimmung der unter den massgebenden Lasten auftretenden maximalen Rissweite (Diagramm GV).

- Der Riss, der sich allenfalls aus der Differenz zwischen Wandend- und Auflager-Verdrehung der Decke ergibt, ist in seiner Grösse nachzuweisen.

404 - Fall $V$

- Bestimmung von $\delta$.

- Ermittlung der Auflagerdrehwinkel infolge der massgebenden Lasten und von Kriechen gemäss 3.4 und infolge von Schwind- und Temperaturverformungen nach 4.5 .

- Wahl des Bemessungsfalles $V$ aufgrund der totalen Auflagerverdrehungen und und Bestimmung der unter der vorhandenen, tatsächlichen Last auftretenden maximalen Risse.

\subsection{Auflagerverdrehung infolge von Schwind- und Temperaturverformungen}

Infolge von Schwind- und Temperaturverformungen werden die Deckenauflager relativ zur Wandaxe verdreht. .Diese Drehwinkel können als Auflagerdrehwinkel aufgefasst werden. 


\section{MBHC}

\section{BE}

\section{Bemessungsfestigkeit $\sigma_{B}\left(\mathrm{~N} / \mathrm{mm}^{2}\right)$}

$\sigma_{B}\left(N / \mathrm{mm}^{2}\right)$

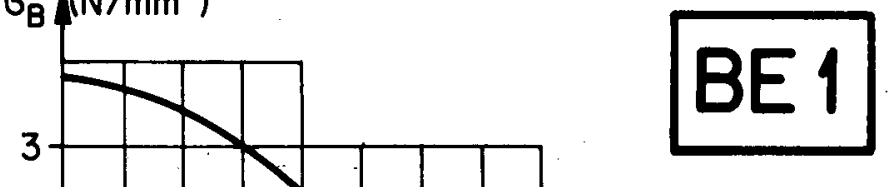

0
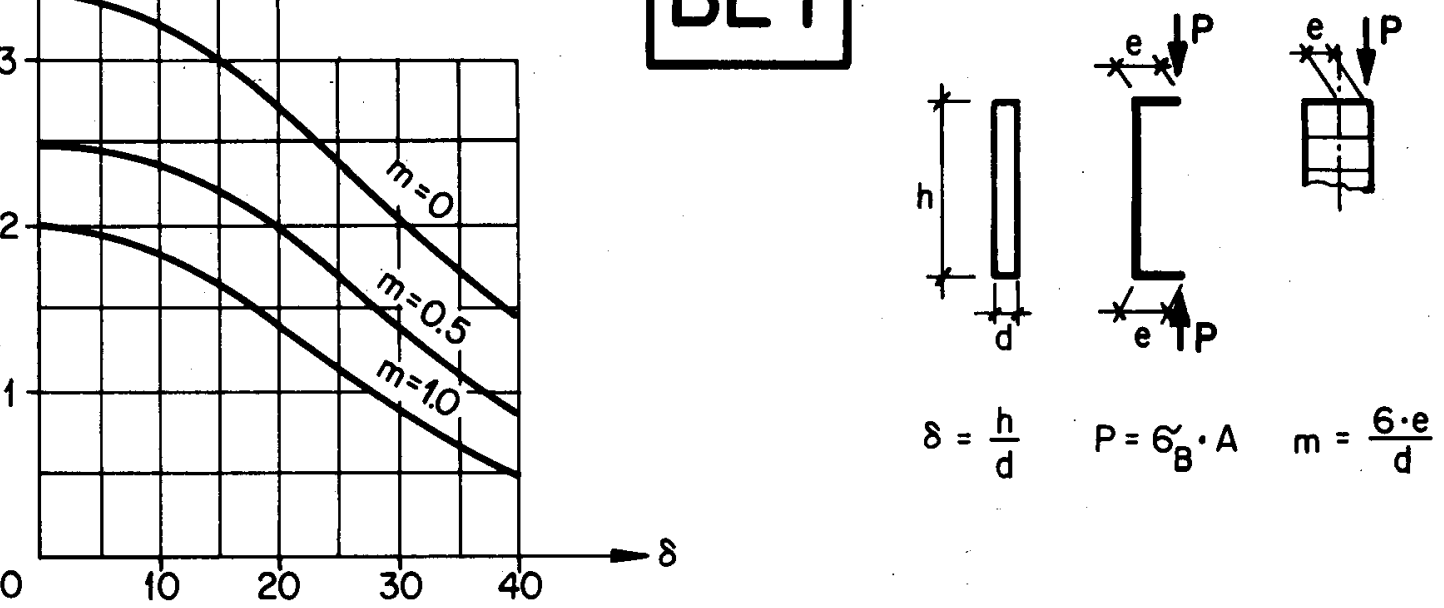

$\sigma_{B}\left\{\left(\mathrm{~N} / \mathrm{mm}^{2}\right)\right.$
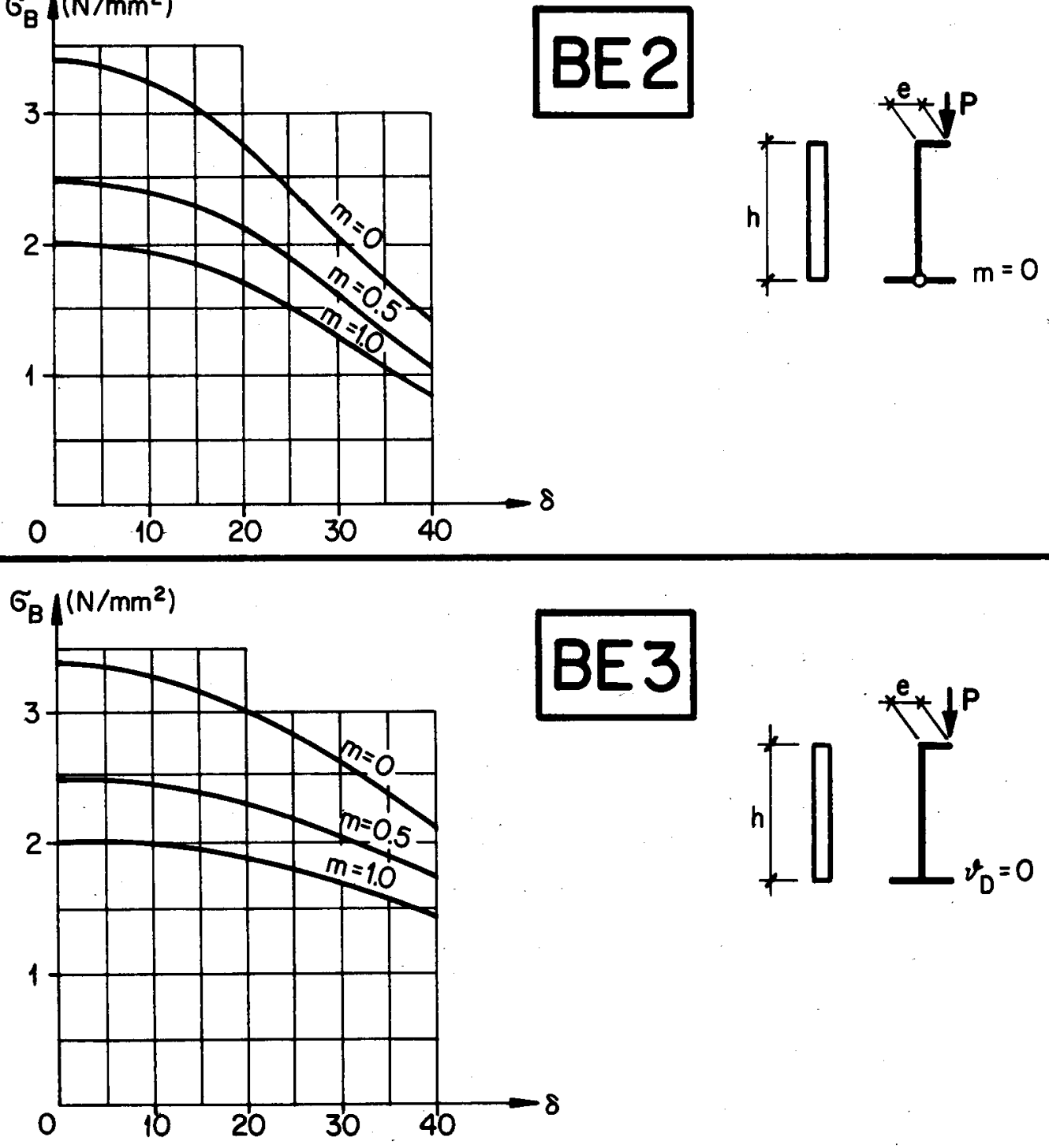


\section{MBHC}

Bemessungsfestigkeit $\sigma_{B}\left(\mathrm{~N} / \mathrm{mm}^{2}\right)$
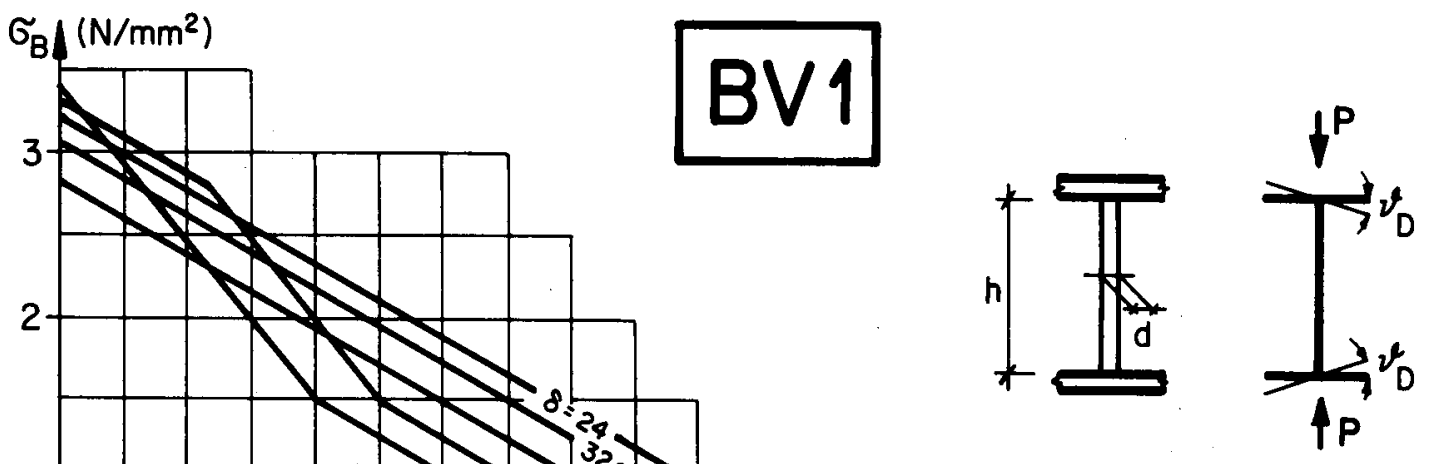

$\delta=\frac{h}{d} \quad P=\sigma_{B} \cdot A$
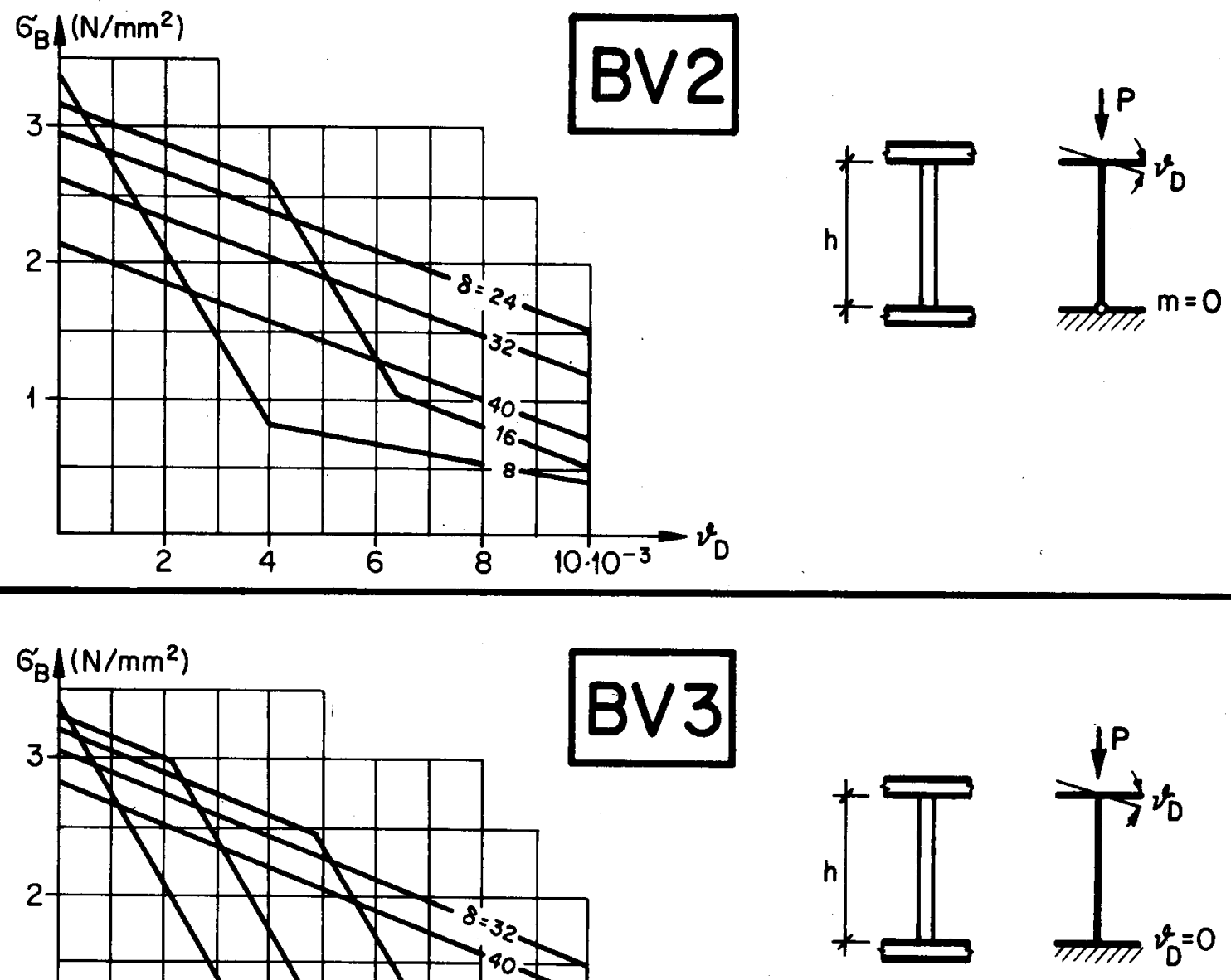


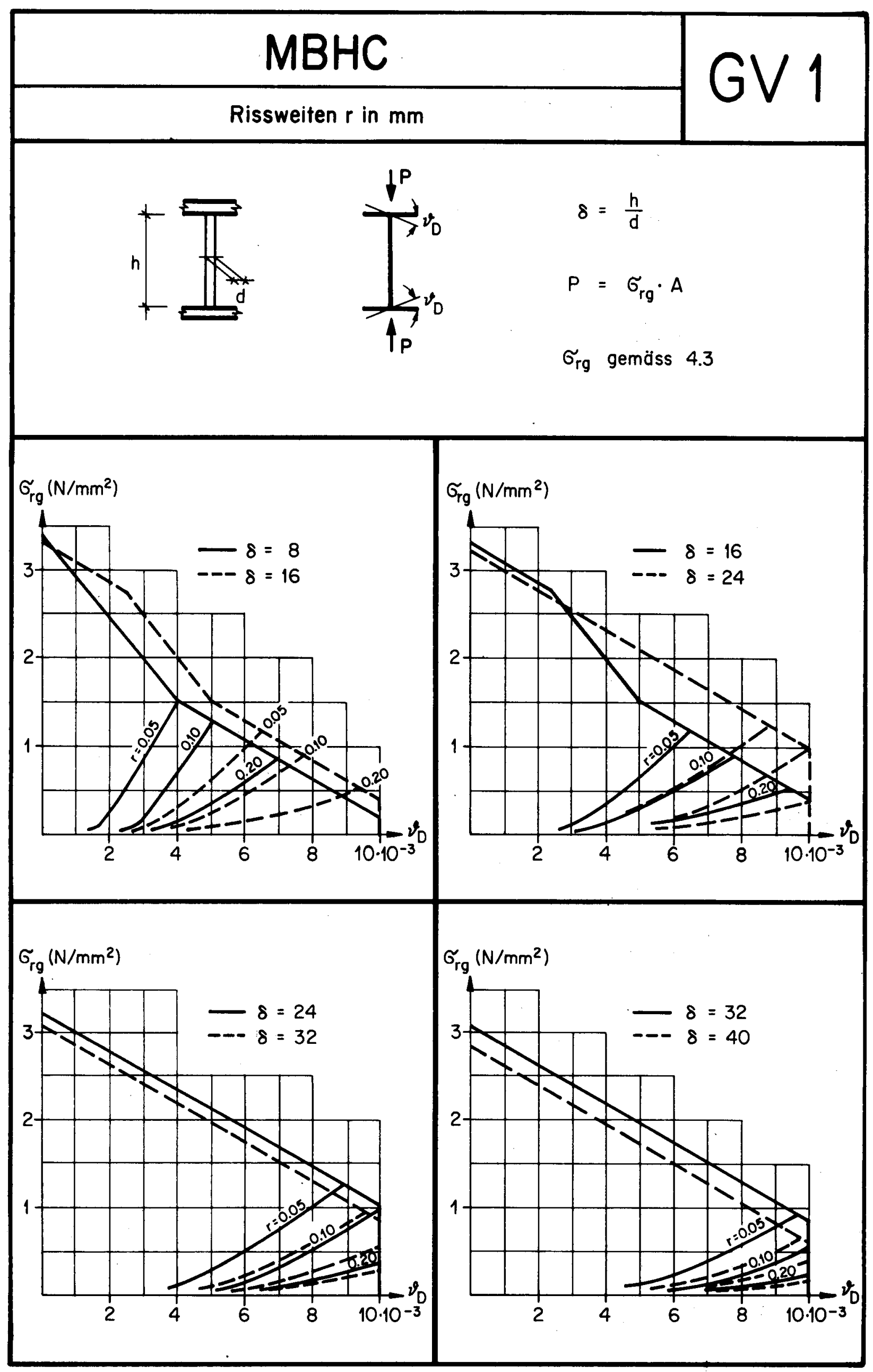




\section{MBHC}

Rissweiten $r$ in $\mathrm{mm}$

\section{GV2}

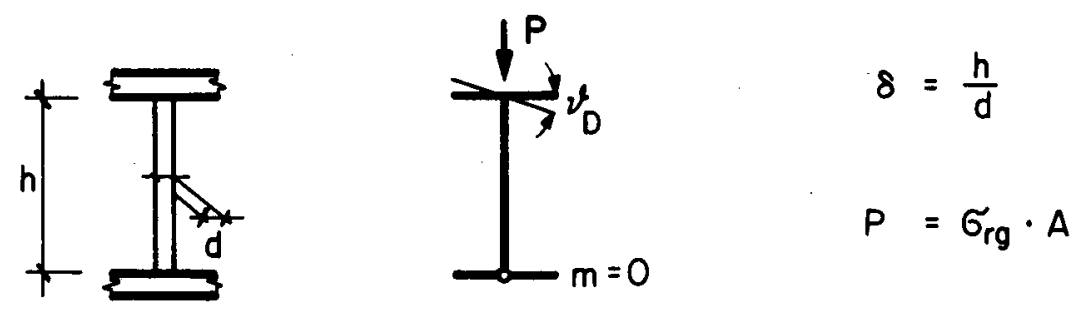

$\sigma_{\text {rg }}$ gemöss 4.3
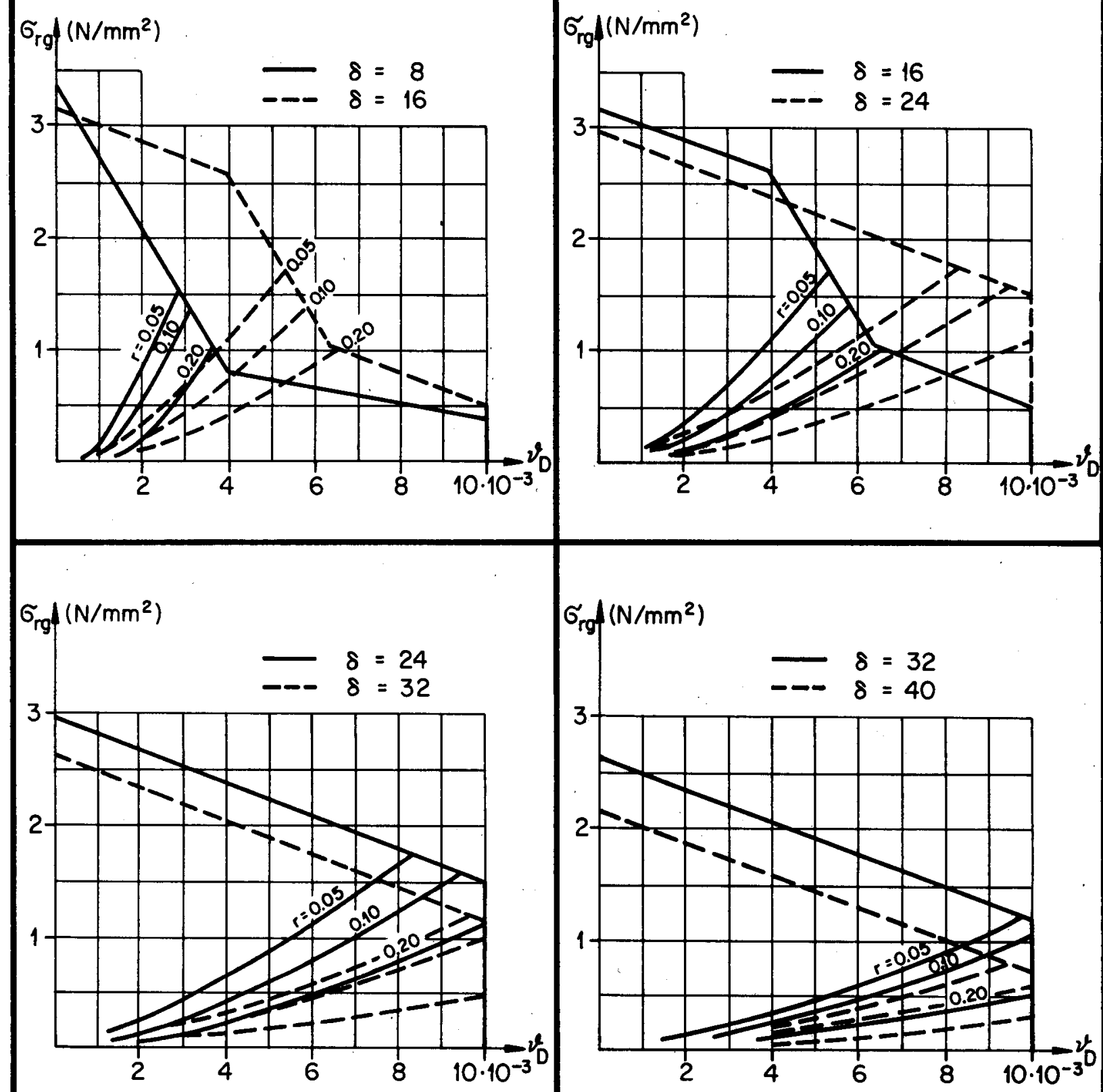


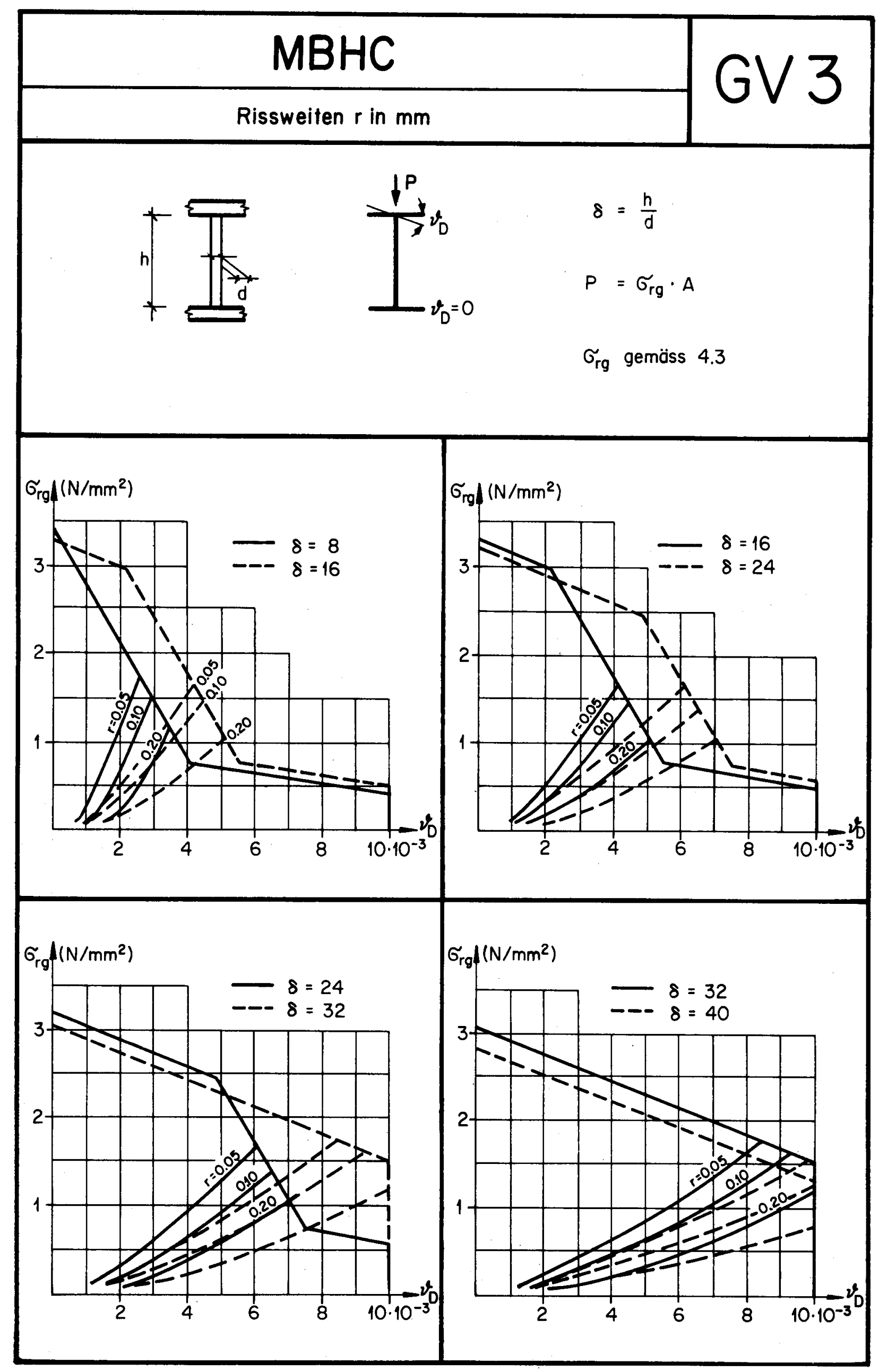




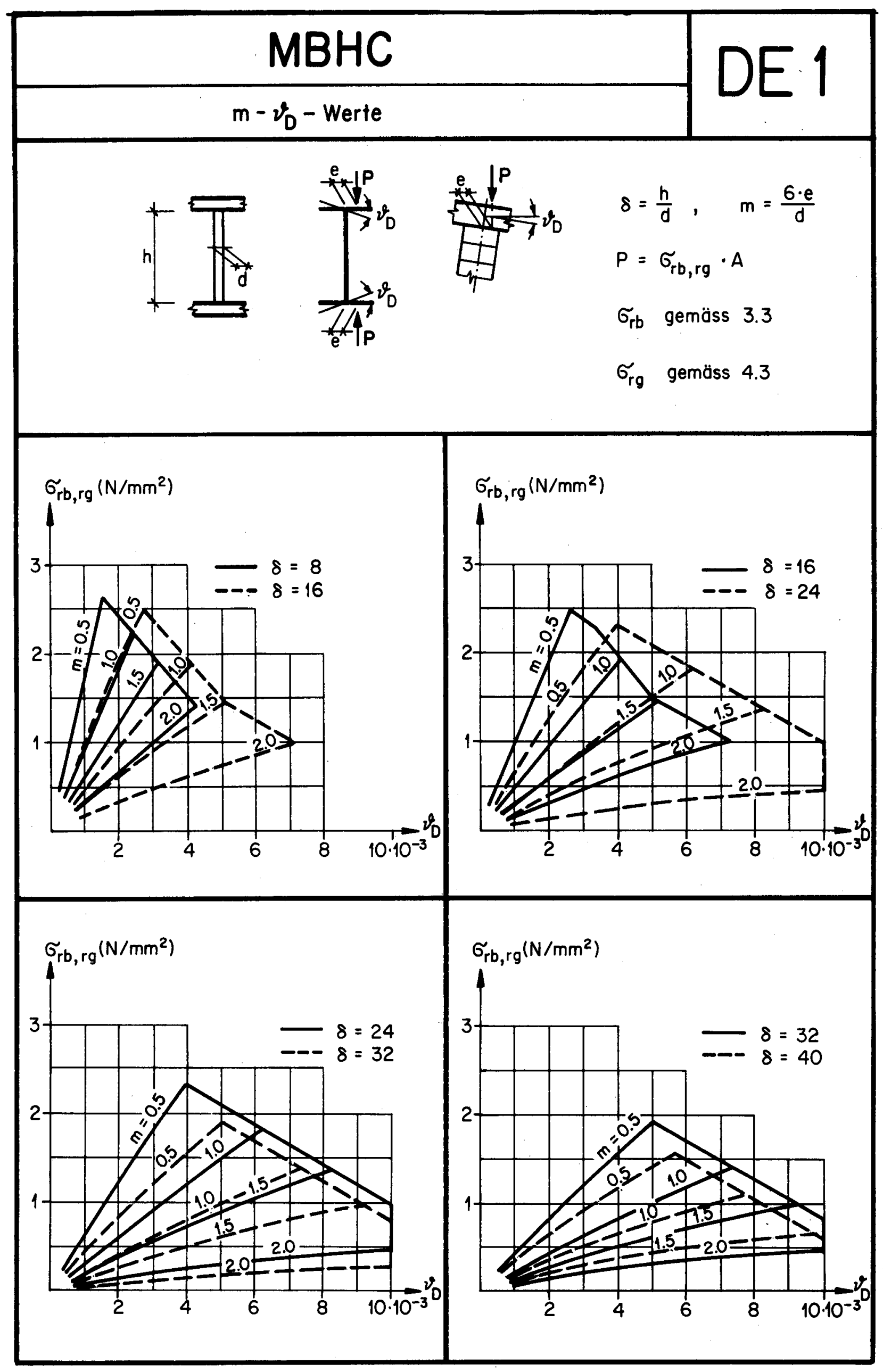




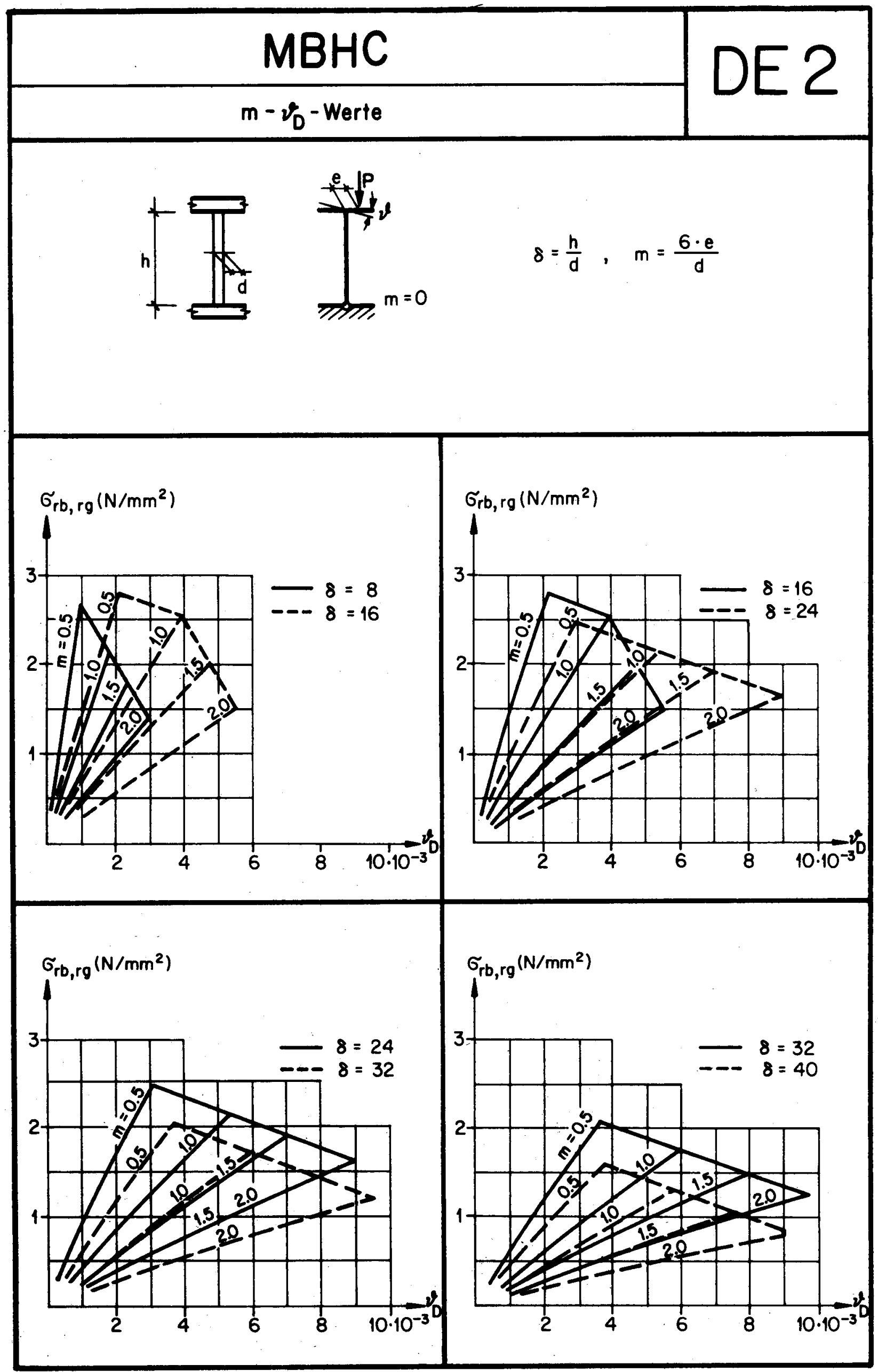




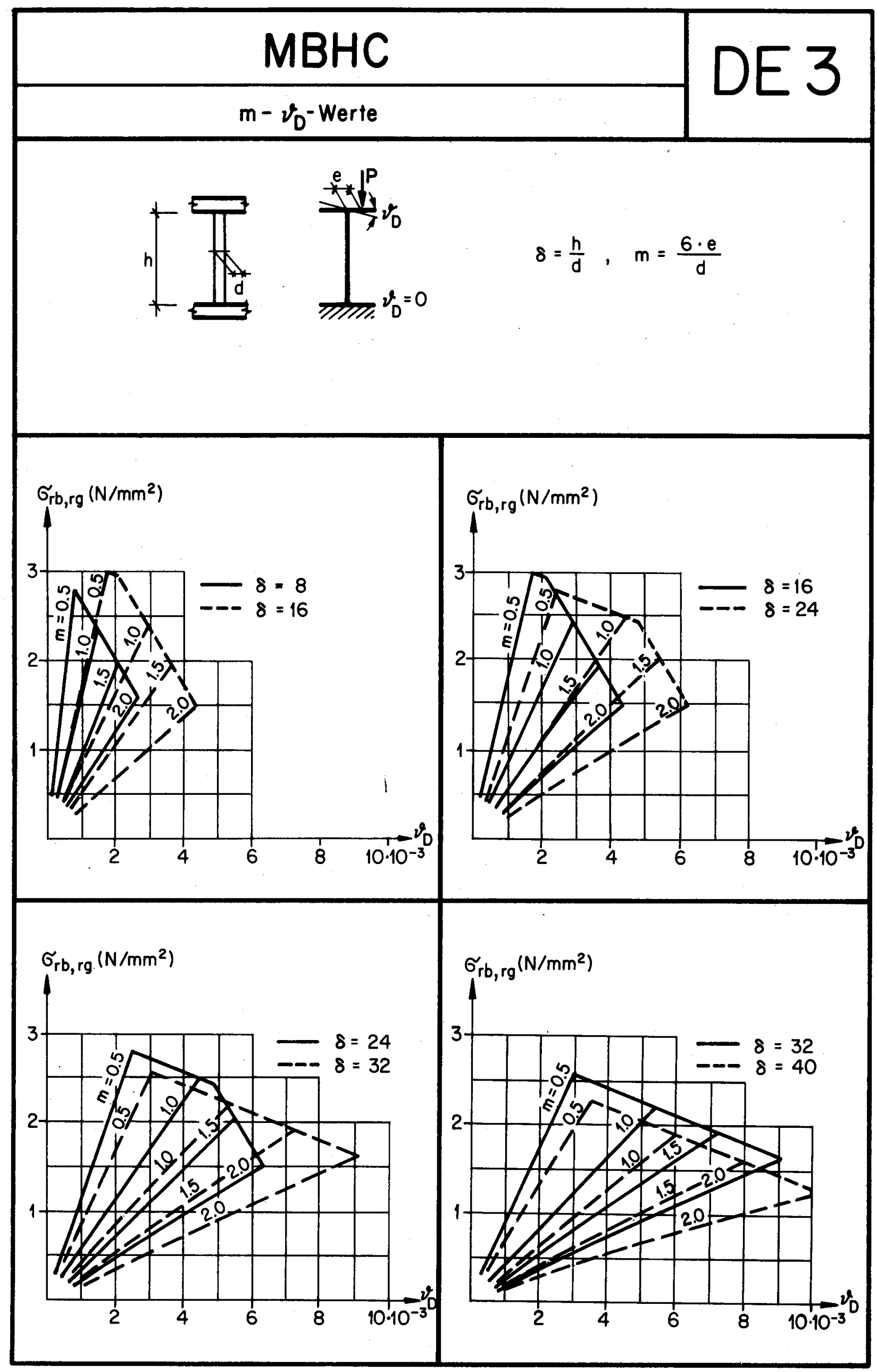

